The Interaction of Katabatic Winds

and Mountain Waves

Gregory Steve Poulos*

* Graduate Research Assistant (Technical Staff Member) at Los Alamos.

Department of Atmospheric Science, Colorado State University,

Fort Collins, Colorado 80523

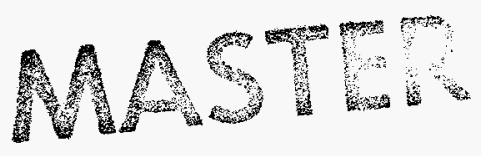




\section{DISCLAIMER}

Portions of this document may be illegible in electronic image products. Images are produced from the best available original document. 


\section{ACKNOWLEDGMENTS}

This dissertation was begun in January, 1993 under an agreement between Los Alamos National Laboratory (LANL) in Los Alamos, New Mexico and Colorado State University (CSU) in Fort Collins, Colorado. For that reason a great number of people were involved, to varying degrees, from those institutions and others. The first and foremost acknowledgment must be for the support of the Department of Energy's Office of Health and Environmental Research (DOE-OHER) who funded the Atmospheric Studies in Complex Terrain (ASCOT) program under which this work was completed. Upon successful defense of this dissertation on August 16, 1996, 3 years and 7 months later, it is remarkable to reflect upon the contributions from others along that long, windy and steep road.

This part of my career was determined by Dr. Jim Bossert at LANL, to whom I give the deepest thanks. He was the champion of my cause and introduced my to the program I would ultimately be supported by - the ASCOT program. As a member of my committee and friend, he was invaluable in brain storming, analysis, encouragement and helping me through some moody moments. By enabling me to do my research work at LANL, Jim's efforts allowed me to be surrounded by perhaps the most intellectually diverse and mentally stimulating group of scientists I will ever encounter.

Also instrumental throughout this process were my co-advisors, Professors Thomas B. McKee and Roger A. Pielke of CSU. Their experience in organizing large projects clarified how I might make a significant contribution to the field of atmospheric science. Their comments and questions were insightful, and they were encouraging as I battled toward completion of the written and oral examinations required for a $\mathrm{Ph}$. D. Dr. McKee's scrutinizing analysis of the mechanisms I have attempted to explain here gave me new perspectives I otherwise would not have considered. My other committee members, Professors Richard Johnson and Jorge Ramirez of Colorado State University, are expressly thanked for their participation in that often time consuming role. They were responsible for keeping me on my toes with some of the tougher questions along the way.

I would also like to thank a number of people at LANL who also contributed to this dissertation. Those are, Dr. J. T. Lee for his help with my mountain wave analysis and explanations of the in's and out's of business at LANL, Dr. William Porch for his insights into katabatic flow, Judy Winterkamp for her computing expertise and support, Dr. Keeley Costigan, John Archuleta, Dr. Jim Kao, Tony Montoya, Lynn McDonald, Deborah Pacheco, Phyllis Tapia and, importantly, Dr. Sumner Barr whose original investigation into external influences on katabatic flow helped form many of the ideas in this dissertation. Finally, in the last year I have made good friends with Dr. Brad Henderson, who in addition to perspective building scientific discussions, also was my faithful 'partner-in-crime' on numerous, stress relieving, fly-fishing trips into the high country of New Mexico in pursuit of the wily brown trout.

While at CSU a number of students and research assistants gave me good advice and guidance. In particular, I would like to thank Dr. Michael Meyers, Dr. Doug Wesley and Dr. Bob Walko for their support throughout this work. Dr. Mike Moran also provided guidance at CSU on dispersion modeling and the RAMS model. Donna Chester and John Kleist were instrumen- 
tal in keeping the computing environment stable. I thank Odie Bliss for her help with contracts and communication. Similarly, Dallas McDonald kept me in line along with her cohort Bryan Critchfield.

The majority of the observational data in this dissertation can be linked to the organizational skills of Timothy Martin, who routinely answered my questions about the ASCOT data hub. Drs. Chris Doran, David Whiteman and Jerome Fast of Pacific Northwest Laboratory are thanked for the many discussions of ASCOT case study days and modeling techniques. Drs. Jerry Schmidt and John Snook of the NOAA Forecast Systems Laboratory were instrumental in accessing a crucial model initialization data set. Their helpful discussions enabled my numerical modeling results to proceed smoothly. From the National Center for Atmospheric Research (NCAR), I would like to thank Ben Bernstein for help with the Winter Icing and Storms Project (WISP) data, Dr. Piotr Smolarkiewicz for helpful terrain-following coordinate modeling discussions and Dr. Terry Clark for information about his modeling of the linear theory of mountain waves. Neither of the latter scientists would have been contacted were it not for introductions and discussions with Dr. Jon Reisner of LANL. Further thanks go out to Dr. Nigel Wood of the United Kingdom Meteorological Office for discussions and documentation of their terrain-following coordinate model.

Although it is likely I have failed to thank some others who helped me bring this dissertation to a happy conclusion, my thanks to them are no less sincere and I appreciate all who were involved. 


\section{TABLE OF CONTENTS}

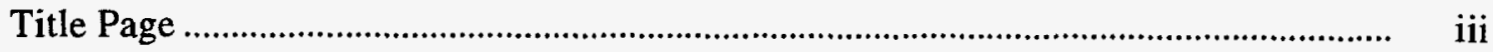

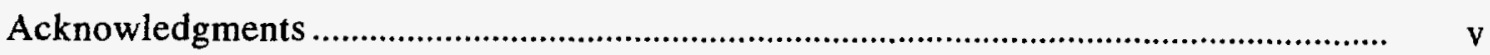

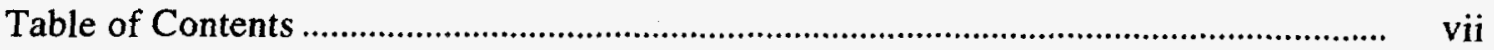

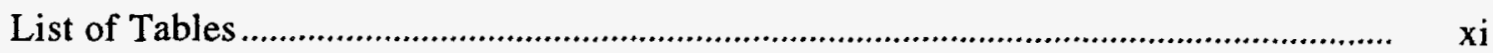

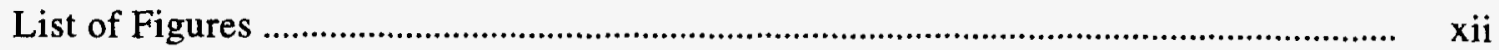

List of Symbols and Acronyms ................................................................................

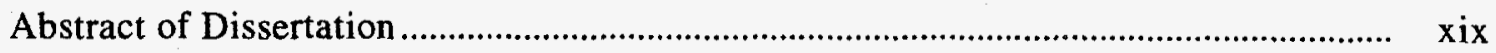

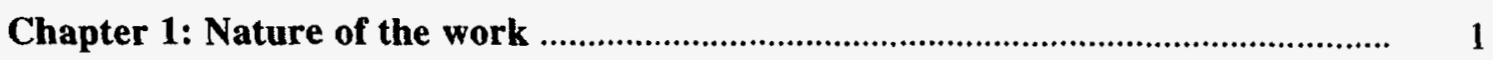

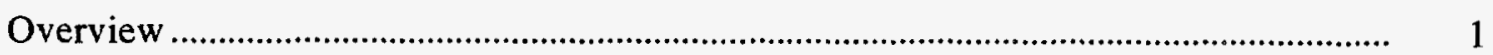

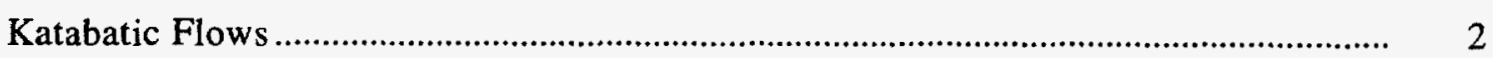

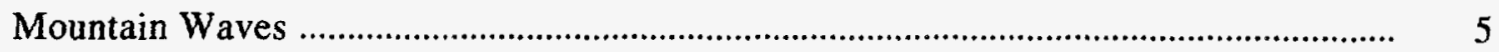

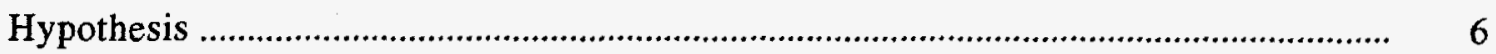

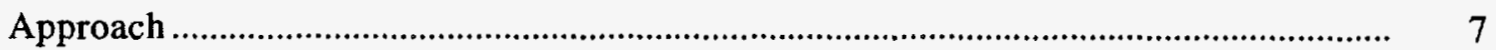

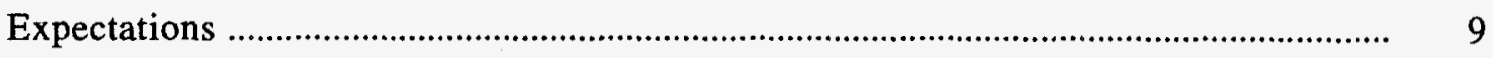

Chapter 2: Previous related work .......................................................................... 10

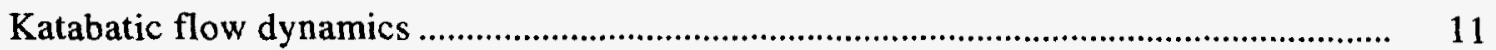

Of downslope flow ........................................................................................... 12

Of down valley flow ........................................................................................ 16

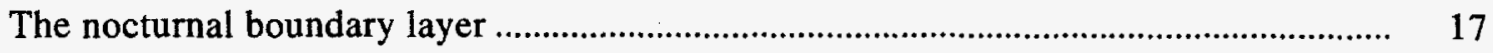

NBL Characteristics ................................................................................... 19

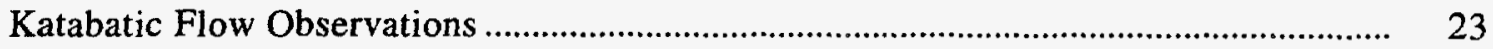

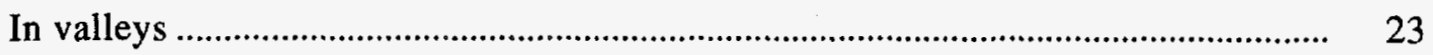

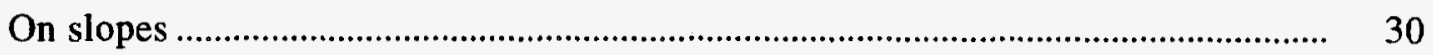

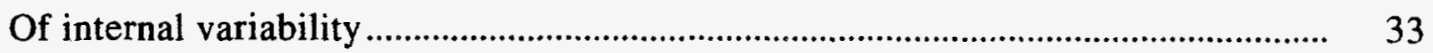

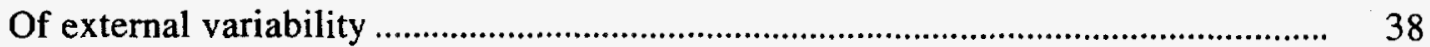

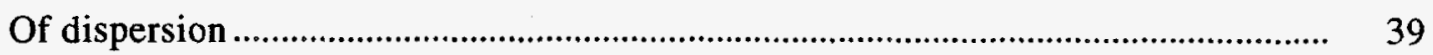

Analytical Solutions for Katabatic Flow ..................................................................... 39

Formulations with vertical dependence ............................................................... 40

Layer average formulations ................................................................................ 47

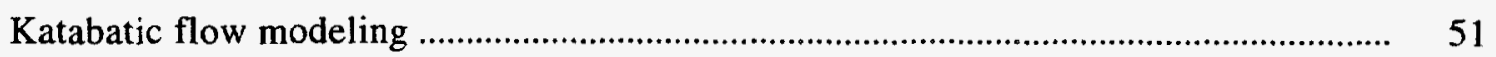

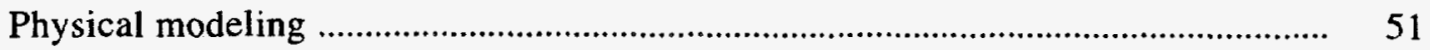

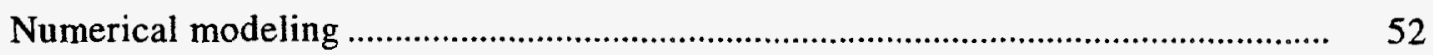

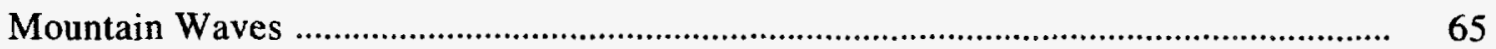

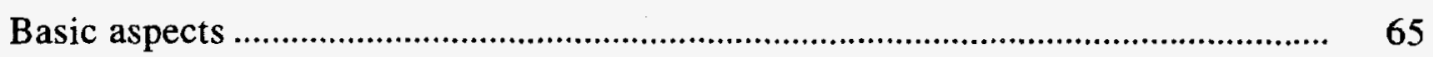

Atmospheric perturbations due to mountain waves ............................................... 68 


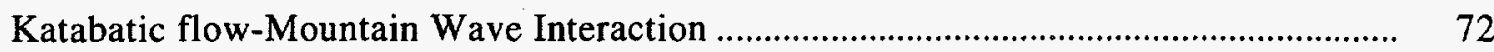

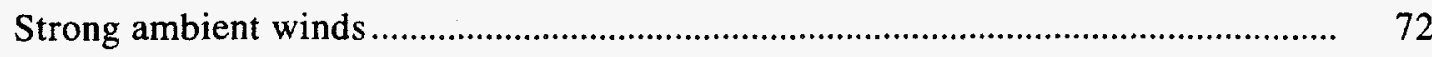

Ambient wind opposing katabatic flow direction ................................................. 73

Ambient wind aiding or following katabatic flow direction ................................. 76

Ambient wind perpendicular to katabatic flow direction ...................................... 78

For off valley axis ambient flow directions ......................................................... $\quad 80$

Variability induced by overlying flow ................................................................ 83

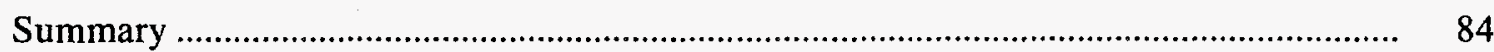

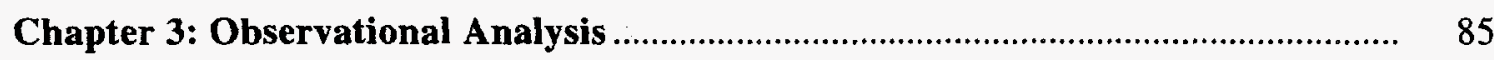

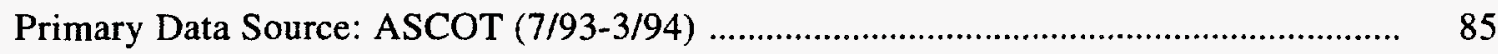

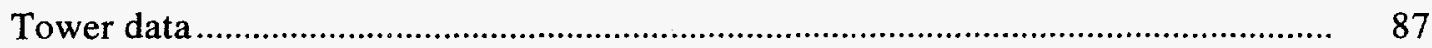

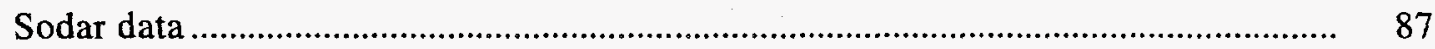

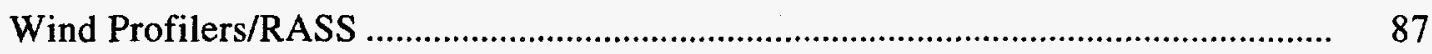

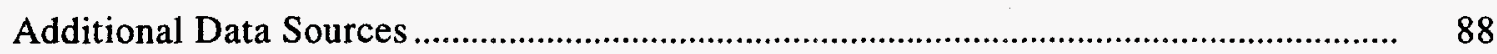

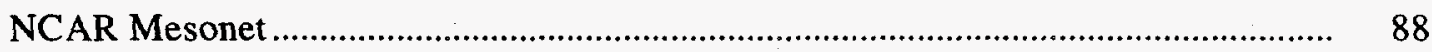

NWS Soundings and Surface Observations .......................................................... 88

NOAA Aeronomy Laboratory Photochemistry Experiment (8-9/93) ...................... 88

Colorado AIRS Sites (6/92-3/94) .................................................................... 89

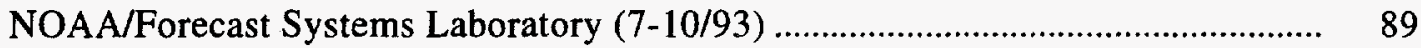

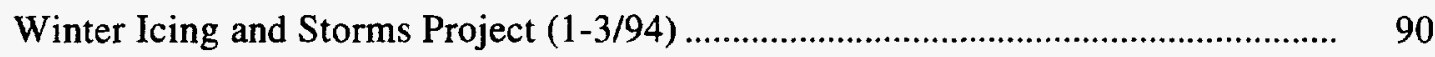

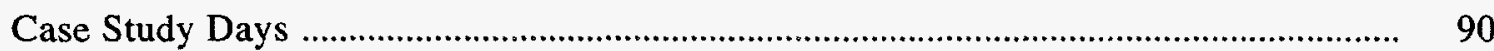

Drainage Flow Characterization ....................................................................... 91

Case 1 - Undisturbed katabatic flow: October 02-03, 1993 ................................... 95

Case 2 - Katabatic flow with $320^{\circ}$ ambient flow: 03-04 September 1993 ................. 96

Case 3 - Katabatic flow perturbed by $225^{\circ}$ flow: 14-15 September 1993 ............... 102

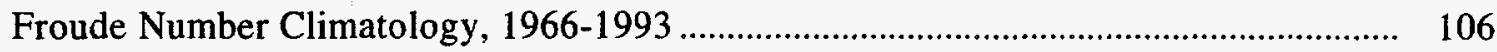

General characteristics ................................................................................ 108

Comparison with ASCOT 1993 Froude numbers ................................................... 110

Observational Contribution to Interaction Concept ................................................ 110

Chapter 4: Model Description and Validation ................................................... 112

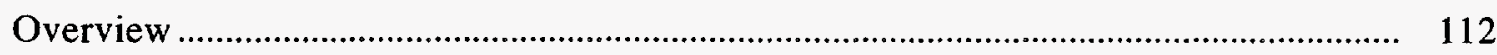

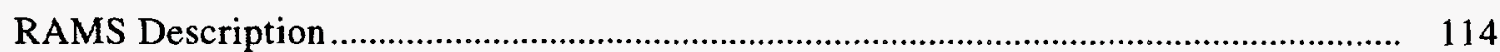

The Application of Rams in Steep Topography ...................................................... 117

Rule of thumb slope maximum criteria ............................................................... 117

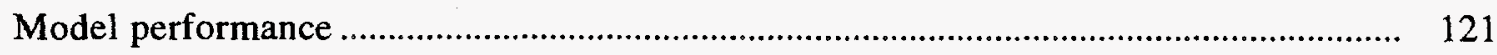

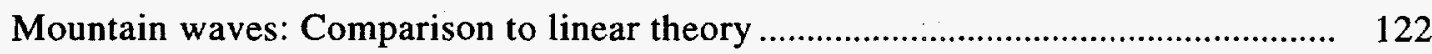


Chapter 5: Idealized Modeling Results

Overview

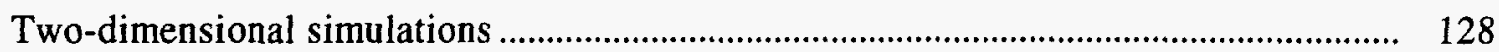

Undisturbed katabatic flow ................................................................................ $\quad 128$

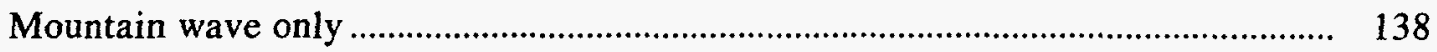

Katabatic flow with mountain wave ............................................................... $\quad 142$

Three-dimensional idealized simulations ........................................................... 150

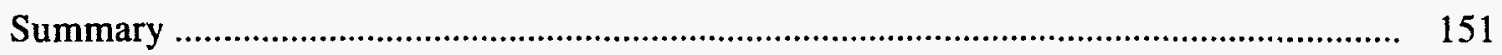

Chapter 6: Realistic Topography Model Results .................................................... 154

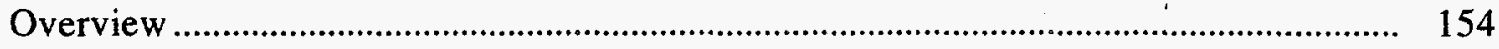

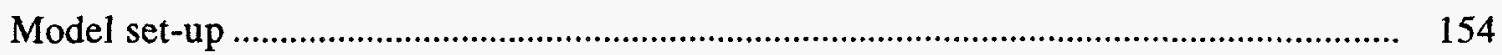

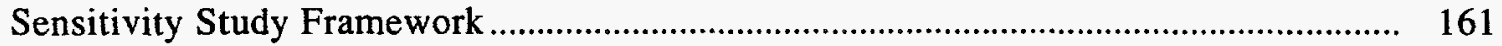

Comparison of the realistic case to observations ........................................................ 165

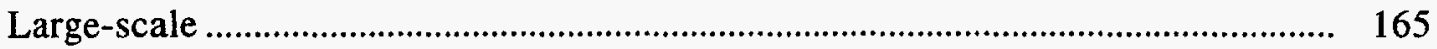

Comparisons with ASCOT observations............................................................. 170

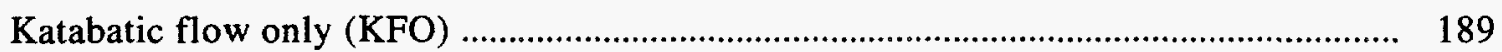

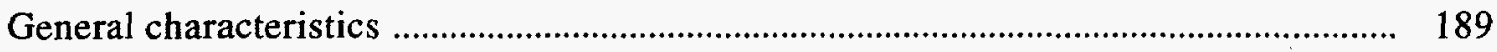

Lower Eldorado Canyon katabatic flow ............................................................... 194

Eldorado Canyon air parcel dispersion .............................................................. 199

Katabatic only simulation summary .................................................................. 201

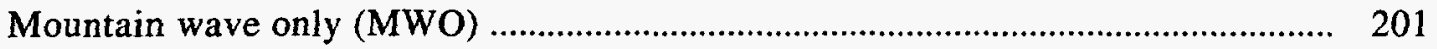

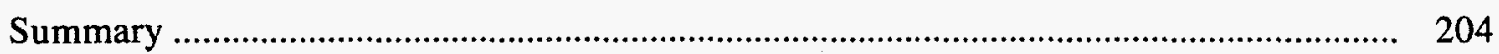

Chapter 7: The Interaction and Evolution of the Phenomena ................................ 206

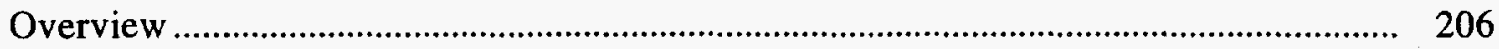

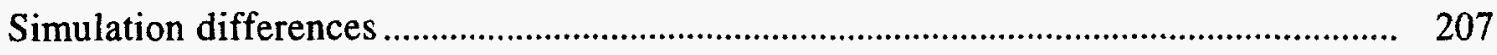

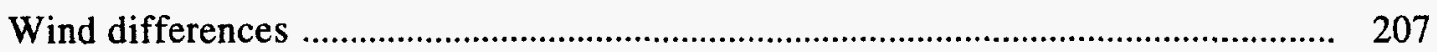

Potential Temperature differences ...................................................................... 213

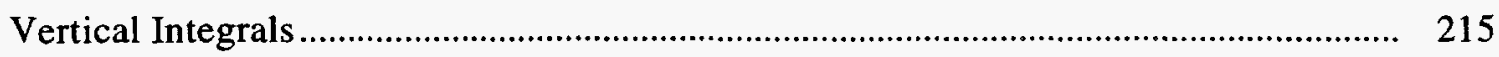

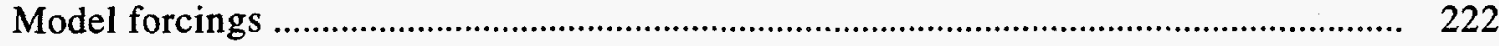

Lower canyon, $50 \mathrm{~m}$ above ground level ............................................................ 223

Lower canyon, $250 \mathrm{~m}$ above ground level .......................................................... 228

Upper canyon, $50 \mathrm{~m}$ above ground level ........................................................... 231

Plains east, $50 \mathrm{~m}$ above ground level .................................................................... 233

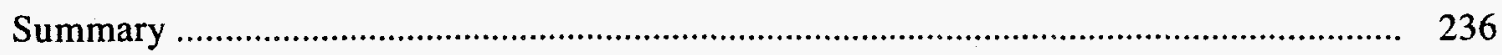


Chapter 8: Conclusions

Overview

Undisturbed katabatic flow

241

The Interaction of Katabatic Flow and Mountain Waves

Turbulent effects

242

Mountain wave induced pressure effects on katabatic flow 244

The effect of evolving thermal stratification on mountain waves 246

Their mutual overnight evolution 247

Other Conclusions 247

Modeling Alpine Phenomena 247

Froude Number Climatology 248

Truncation Errors in $\sigma$-coordinate models 248

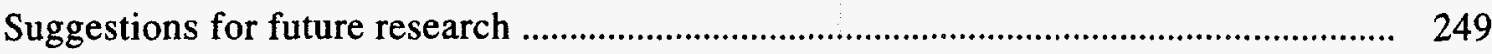

References 252

Appendix A: ASCOT 1993 Katabatic Flow Nights Summary 276 Appendix B: Froude Number Climatology 1966-1993 289 


\section{LIST OF TABLES}

Table 3.1 The top 30 likely katabatic flow nights.

Table 3.2 Hour average Rim Rock tower readings.

Table 4.1 Model parameters used by Clark (1977).

Table 5.1 A summary of two-dimensional simulations.

Table 5.2 Katabatic flow details at 8 hours.

Table 6.1 Relevant scales, required grid spacing and actual grid spacing for MKI.

$\begin{array}{ll}\text { Table 6.2 } & \text { Model configuration details for case study simulations } \\ \text { Table 6.3 } & \text { Model information by grid for case study simulations. }\end{array}$

Table 6.4

ASCOT 1993 observational sites used for comparisons with RAMS. .

160

Table 6.5

Average $\mathrm{Fr}_{u}$ by month of year.

173

Table 6.6

Average thermal stability and wind speed below barrier by month.

291

Table 6.7 Average $\mathrm{Fr}_{\mathrm{v}}$ by month of year. 


\section{LIST OF FIGURES}

Figure 1.1: The conceptual model of katabatic slope flow of Manins (1992).

Figure 1.2: $\quad$ ASCOT time-height observations of katabatic flow at the head of Coal Creek Canyon on 02-03 October 1993 by sodar.

Figure 1.3: Idealized streamlines, surface pressure perturbation and wind speed perturbation from linear theory of mountain wave flow.

Figure 1.4: Observations of potential temperature structure (solid lines), cloud and significant turbulence $(+)$ during a severe wind storm.

Figure 2.1: a) temperature $\left({ }^{\circ} \mathrm{C}\right)$, and b) pressure $(\mathrm{mb})$ and wind vectors, from a numerical simulation of radiatively driven flow on a $2.5^{\circ}$ slope that apply Bjerknes' circulation theorem to describe katabatic slope winds.... 14

Figure 2.2: The diurnal cycle of valley winds for clear sky, quiescent conditions as diagrammed by Defant (1949).

Figure 2.3: The diurnal cycle of near surface conditions during clear sky, light wind conditions (Stull 1991).

Figure 2.4: Observed mean (for 01-06 LST) vertical profiles of a) temperature (K) and b) wind speed $\left(\mathrm{m} \mathrm{s}^{-1}\right)$ in the down-valley direction of Brush Creek Valley, Colorado on four different nights.

Figure 2.5: A schematic of the break up of a temperature inversion in a valley from Whiteman and McKee (1982).

Figure 2.6:

The terrain slope studied by Manins and Sawford (1979b).

Figure 2.7:

Numerical and analytical model solutions for katabatic flow.

Figure 2.8:

Results from the first attempt at numerically modeling katabatic flows

from Thyer (1966).

A realistic katabatic flow simulation by a hydrostatic model. ................... 56

Figure 2.9:

Froude number $=1.0$ flow over a $1 \mathrm{~km}$ high obstacle.

Figure 2.11: A comparison of figures a) and b) from Cornfeld (1938) for two ways aiding flow can impact katabatic slope flow and for c) opposing and d) aiding ambient winds overlying katabatic flow from Mursch-Radlgruber (1995).

Figure 2.12: Drainage flow speed versus height for Coal Creek Canyon, Colorado based on 3 year averages of sodar data.

Figure 2.13:

Variation in depth of penetration normalized by valley depth for

different Froude numbers (Lee et al. 1987).

Figure 2.14: The change in katabatic valley flow structure for a) fixed orientation, $60^{\circ}$ left of directly up-valley at various speeds and, b) at $4 \mathrm{~m} \mathrm{~s}^{-1}$ for various orientations relative to the up valley axis.

Figure 2.15: Variation in depth of drainage flow in Brush Creek Valley for different Froude numbers as calculated from observations (Barr and Orgill 1989). 83

Figure 3.1: Observational sites in and around the two main canyons of interest to the 1993 ASCOT experimental campaign.

Figure 3.2:

Synoptic conditions at $500 \mathrm{mb}$ for a) $00 \mathrm{Z}$, and b) $12 \mathrm{Z}, 4 \mathrm{Sep} 1993$.

As in Figure 3.2 but for $700 \mathrm{mb}$ at a) $00 \mathrm{Z}$, and b) $12 \mathrm{Z}$, 4 Sep $1993 . \ldots \ldots .100$

$\begin{array}{lll}\text { Figure 3.4: } & \text { Coal Creek Canyon sodar observations from } 1600-0900 \text { LST.............. } 103 \\ \text { Figure 3.5: } & \text { ASCOT observations of katabatic flow with mountain wave interaction }\end{array}$ at the head of Coal Creek Canyon on 14-15 Sep 1993 by sodar. ........... 104 
Figure 4.1: Vertical velocity for a) the steady-state solution of linear mountain wave theory from Clark (1977) and b) using RAMS.

Figure 5.1: Model results from the lower portion of the eastern slope in the 2-dimensional, $2.5^{\circ}$ slope simulation.

Figure 5.2: Time-height plots halfway down the eastern slope of the 3-dimensional, $2.5^{\circ}$ slope katabatic flow only simulation.

Figure 5.3: The acceleration balance of $u$ component wind for the 3-d, $2.5^{\circ}$, katabatic flow only simulation.

Figure 5.4: $U$ component wind speed contours at 3 hours into the, a) $\mathrm{Fr}=0.25$, b) $\mathrm{Fr}=0.50, \mathrm{c}) \mathrm{Fr}=0.75$ and d) $\mathrm{Fr}=1.0$, MWO 2-dimensional simulations.

Figure 5.5: Potential temperature contours at 3 hours into the, a) $\mathrm{Fr}=0.25$, b) $\mathrm{Fr}=0.50, \mathrm{c}) \mathrm{Fr}=0.75$ and d) $\mathrm{Fr}=1.0$, MWO 2-dimensional simulations.

Figure 5.6: $\quad U$ component wind speed contours at 9 hours into the idealized 2-dimensional MKI simulations for, a) $\mathrm{Fr}=0.25$, b) $\mathrm{Fr}=0.50$,

c) $\mathrm{Fr}=0.75$ and d) $\mathrm{Fr}=1.0$.

Figure 5.7: Potential temperature contours at 9 hours into the idealized

2-dimensional $\mathrm{MKI}$ simulations for, a) $\mathrm{Fr}=0.25$, b) $\mathrm{Fr}=0.50$,

c) $\mathrm{Fr}=0.75$ and d) $\mathrm{Fr}=1.0$.

Figure 5.8: $\quad U$ component wind speed contours at 12 hours into the idealized 2-dimensional $\mathrm{MKI}$ simulations for, a) $\mathrm{Fr}=0.25$, b) $\mathrm{Fr}=0.50$, c) $\mathrm{Fr}=0.75$ and d) $\mathrm{Fr}=1.0$.

Figure 5.9:

Potential temperature contours at 12 hours into the idealized

2-dimensional MKI simulations for, a) $\mathrm{Fr}=0.25$, b) $\mathrm{Fr}=0.50$,

c) $\mathrm{Fr}=0.75$ and d) $\mathrm{Fr}=1.0$.

Figure 5.10: East-west vertical cross sections through the center of the

3-dimensional idealized, $\mathrm{Fr}=0.50$, mountain wave forced simulations.

Figure 6.1:

The 5 grids used in the realistic simulations.

Figure 6.2: RAMS topographical representations for each of the 5 grids.

Figure 6.3:

a) wind speed and b) direction, c) standard deviation of wind direction, and d) temperature, for the $300 \mathrm{~m}$ Boulder Atmospheric Observatory from 1700 MST 3 Sep 1993 to 0500 MST 4 Sep 1993 at 4 levels.

Figure 6.4: Wind vectors and pressure ( $1.0 \mathrm{mb}$ increments) on a horizontal plane $5518 \mathrm{~m}$ MSL for a) $2100 \mathrm{MST}, 3$ Sep 1993 and b) $0200 \mathrm{MST}$, 4 Sep 1993.

Figure 6.5: Wind vectors and pressure (1.0 mb increments) on a horizontal plane 2973 m MSL for a) 2100 MST, 3 Sep 1993 and b) 0200 MST, 4 Sep 1993.

Figure 6.6: Observed wind speed and direction at the Gross Reservoir profiler RAMS modeled wind speed and direction for the Gross Reservior profiler somewhat similar to observations in Figure 6.6.

Figure 6.8: a) wind speed, b) wind direction and c) vertical motion as observed by the Militzer sodar for the overnight period 3-4 Sep 1993.

Figure 6.9:

a) wind speed, b) wind direction and c) vertical motion as modeled by RAMS for a location near the Militzer sodar for the overnight period 3-4 Sep 1993. 
Figure 6.10: a) wind speed, b) wind direction and c) vertical motion as

observed by the Robinson sodar for overnight 3-4 Sep 1993.

Figure 6.11: a) wind speed, b) wind direction and c) vertical motion as modeled by RAMS for a location near the Robinson sodar for the overnight period 3-4 Sep 1993.

Figure 6.12: a) wind speed, b) wind direction and c) vertical motion as modeled by RAMS for a location near the NREL sodar for the overnight period 3-4 Sep 1993.

Figure 6.13: a) wind speed, b) wind direction and c) vertical motion as modeled by RAMS for a location near the Ruthrauff sodar for the overnight period 3-4 Sep 1993.

Figure 6.14: Observed and modeled 1-hour average a) wind speed, b) wind direction and c) temperature at the Lois Ranch ASCOT observational site for the overnight period 3-4 Sep 1993.

Figure 6.15:

As in Figure 6.14 but for the Pats Flat ASCOT observational site..........

Figure 6.16:

As in Figure 6.14 but for the Rex Ranch ASCOT observational site. .....

Figure 6.17: Horizontal wind vectors and topography (100 m contours) for $50 \mathrm{~m}$

AGL at midnight of the 3-4 Sep 1993 case night for an idealized, no wind initialization.

Figure 6.18: Plots of the variables that show the cause of the wind maximum at the peak of the mountain just to the north of Eldorado Canyon at midnight of KFO.

Four east-west vertical cross sections down the center of Eldorado

Figure 6.19: Four east-west vertical cross sections
Canyon at $0700 \mathrm{Z}$ (midnight LST).

Four north-south vertical cross sections for a location about $1.2 \mathrm{~km}$

Figure 6.20:

up-canyon from the exit of Eldorado Canyon.

Figure 6.21:

Particle trajectories from 5 sources within Eldorado Canyon at 3 hours into a LPDM simulation of the katabatic flow only case.

East-west cross sections of potential temperature $(0.5 \mathrm{~K}$ intervals) and wind vectors through Eldorado Canyon on Grid 4.

Figure 6.22:

Two-minute data interval, east-west component winds versus time at

Figure 7.1: three different horizontal locations on Grid 5.

Figure 7.2:

The same as Figure 7.1 but for v-component.

Figure 7.3:

The same as Figure 7.1 but for w-component.

Figure 7.4:

The same as Figure 7.1 but for potential temperature..

Figure 7.5:

The upwardly summed vertical integrals of westerly mass flux. ..............

The same as Figure 7.5 but for vertical mass flux...

Figure 7.6:

Figure 7.7:

The same as Figure 7.5 but for heat content (megajoules $\mathrm{m}^{-2}$ ) integrated downward relative to the model start at 1700 MST.

Figure 7.8:

Terms of the conservation of $\mathbf{u}$-component momentum equation as calculated by RAMS for the 3-4 Sep 1993 case night.

An expanded scale plot of the $u$ component at the lower canyon location for 12 hours during the realistic simulation.

Figure 7.9:

Figure 7.10:

As in Figure 7.8 but for $250 \mathrm{~m}$ AGL (vertical level 15). 
Figure 8.1: A schematic diagram of the influence of a mountain wave descending into lee side katabatic flows.

Figure 8.2: Schematic diagrams of the two dynamic pressure effects of mountain waves on katabatic flow.

Figure B.1: Froude number histogram based on all soundings at Grand Junction for the period 1966-1993.

Figure B.2:

As in Figure B. 1 but limited to the spring season.

Figure B.3:

As in Figure B.1 but for the summer season..

293

Figure B.4:

As in Figure B.1 but for autumn.

294

Figure B.5:

Figure B.6:

As in Figure B.1 but for the winter season .

294

As in Figure B. 1 but for the horizontal wind, V, for only those days where the westerly componen was at least half the flow.

Figure B.7: As in Figure B.1 but for the spring season only

Figure B.8: As in Figure B.1 but for the summer season. 


\section{LIST OF SYMBOLS AND ACRONYMS}

\section{Acronyms}

ASCOT - Atmospheric Studies in Complex Terrain

BAO - Boulder atmospheric observatory

CBL - convective boundary layer

FSL - Forecast Systems Laboratory

MAPS - Mesoscale Analysis and Prediction System

MKI - mountain wave/katabatic flow interaction

NCAR - National Center for Atmospheric Research

NOAA - National Oceanic and Atmospheric Administration

NBL - nocturnal boundary layer

RASS - radio acoustic sounding system

TFCS - terrain following coordinate system

\section{Symbols}

$B_{1,2,3 \text { and } 4}-$ constants in Jeffreys (1922) analytical solution for katabatic flow

C - circulation $\left(\mathrm{m}^{2} \mathrm{~s}^{-2}\right)$ or Celsius degrees

- fraction of sky covered by cloud in Ye et al. (1989)

Fr - Froude number

$\mathrm{Fr}_{\mathrm{u}}$ - Froude number calculated using the east-west horizontal wind component

$\mathrm{Fr}_{\mathrm{v}}$ - Froude number calculated using the total horizontal wind

$\mathrm{H}$ - barrier height in the calculation of Froude number or layer depth

$\mathrm{K}$ - Kelvin temperature or eddy viscosity $\left(\mathrm{m}^{2} \mathrm{~s}^{-2}\right)$

$\mathrm{K}_{\mathrm{H}}$ - eddy viscosity for heat

$\mathrm{K}_{\mathrm{M}}$ - eddy viscosity for momentum

$N$ - Brunt-Vaisala frequency

$\mathrm{R}$ - the gas constant for dry air, $287 \mathrm{~J} \mathrm{~kg}^{-1} \mathrm{~K}^{-1}$

$\mathrm{S}$ - surface area or ridge separation

$\mathrm{T}$ - temperature $\left({ }^{\circ} \mathrm{C}\right.$ or $\left.\mathrm{K}\right)$

$\mathrm{U}$ - Mean east-west wind component

W - watts (energy/time) or ridge width

a - slope angle

$c_{1}$ - a constant in Jeffreys (1922) analytical solution

g - gravitational acceleration, $9.81 \mathrm{~m} \mathrm{~s}^{-2}$

1 - Scorer parameter or sin (slope angle) in Jeffreys' (1922) analytical solution or a parameter in Prandtl's (1942) analytical solution

$\mathrm{mb}$ - millibar(s) (100 Pascals of pressure)

p - atmospheric pressure

$\mathrm{t}-$ time

$\mathrm{z}$ - altitude above ground level

$z_{o} \quad$ - roughness length 


\section{Greek symbols}

$\Delta-$ change in a variable relative to the standard value

$\Lambda$ - a factor defined in Jeffreys (1922) analytical solution

$\Omega$ - a factor defined in Jeffreys (1922) analytical solution

$\alpha$ - slope angle

$\alpha_{\mathrm{a}}$ - coefficient of expansion of air used in Jeffreys (1922) analytical solution for katabatic slope flow

$\varphi$ - a parameter defined in Jeffreys (1922) analytical solution

$\lambda$ - deviation of ambient $\theta$ lapse rate from the adiabatic lapse rate

$\mu$ - frictional constant

$\theta$ - potential temperature

$\theta^{\prime}$ - potential temperature deviation

$\theta_{0}-$ ambient potential temperature in Prandtl's (1942) analytical solution

$\rho$ - density $\left(\mathrm{kg} \mathrm{m}^{-3}\right)$

$\sigma$ - unit of area

$\sigma_{\theta}$ - standard deviation of wind direction in degree 


\title{
THE INTERACTION OF KATABATIC WINDS AND MOUNTAIN WAVES
}

by

\author{
Gregory Steve Poulos
}

\begin{abstract}
The variation in the oft-observed, thermally-forced, nocturnal katabatic winds along the east side of the Rocky Mountains can be explained by either internal variability or interactions with various other forcings. Though generally katabatic flows have been studied as an entity protected from external forcing by strong thermal stratification, this work investigates how drainage winds along the Colorado Front Range interact with, in particular, topographically forced mountain waves. Previous work has shown, based on measurements taken during the Atmospheric Studies in Complex Terrain 1993 field program, that the actual dispersion in katabatic flows is often greater than reflected in models of dispersion.

The interaction of these phenomena is complicated and non-linear since the amplitude, wavelength and vertical structure of mountain waves developed by flow over the Rocky Mountain barrier are themselves partly determined by the evolving atmospheric stability in which the drainage flows develop. Perturbations to katabatic flow by mountain waves, relative to their more steady form in quiescent conditions, are found to be caused by both turbulence and dynamic pressure effects. The effect of turbulent interaction is to create changes to 1) katabatic flow depth, 2) katabatic flow speed, 3) katabatic jet height and, 4) vertical thermal stratification. The pressure effect is found to primarily influence the variability of a given katabatic flow through the evolution of integrated column wave forcing on surface pressure. Variability is found to occur on two scales, on the mesoscale due to meso-gamma scale mountain wave evolution, and on the microscale, due to wave breaking. Since existing parameterizations for the statically stable case are predominantly based on nearly flat terrain atmospheric measurements under idealized or nearly quiescent conditions, it is no surprise that these parameterizations often contribute to errors in prediction, particularly in complex terrain.
\end{abstract}




\section{CHAPTER 1}

\section{NATURE OF THE WORK}

"...remnants of cold air in valleys form closed systems and may be excited into oscillations (similar to waves on lakes) by the passage of a foehn current over them. These oscillations show up as temperature fluctuations that are not to be confused with the short, periodic pressure fluctuations that occur during foehn."

- F. Defant, 1951, paraphrasing the work of A. Defant from the first decade of the 20 th century

\subsection{OVERVIEW}

This work has its genesis in questions that arise from observations of katabatic flows from the Atmospheric Studies in Complex Terrain (ASCOT) program. Also called drainage flows, these winds are generated over slopes and in valleys (not necessarily sloped valleys) by surface-based radiative cooling. Their investigation with observational, theoretical and numerical techniques, has mostly focused on the physics of their generally undisturbed evolution, with some exceptions. The majority of observational studies, however, reveal the non-idealized nature of these flows; katabatic flows are not disconnected from external influences. Despite these influences, primarily larger scale winds or clouds, katabatic flows are a persistent overnight feature over the Earth's land and ice masses where any slope exists. It is the externally-induced deviation from an undisturbed or steady-state katabatic evolution that will be investigated here.

Of the many influences on katabatic winds, this work will only attempt to shed light on one: mountain waves (one form of mountain wave phenomena, the foehn, is mentioned in 
the introductory quote). The reason this particular phenomena was selected was two-fold, 1) both katabatic and mountain wave flows occur frequently and simultaneously in complex topography, and 2) observed variability of katabatic flows in mountainous terrain is potentially caused, in part, by terrain-induced internal waves (mountain waves). This variability can be a serious problem for forecasts of pollutant dispersion, turbulence and general weather conditions in many regions (i.e. Poulos and Bossert, 1995). Egan (1984) notes, in a review of transport and diffusion, that the interaction of katabatic flows with ambient flow is not properly understood.

\subsection{Katabatic Flows}

Figure 1.1, from Manins (1992) shows the basic configuration of an undisturbed katabatic slope flow. On a slope of angle $\alpha$, an inversion condition of depth $h$ develops due to surface radiational cooling and turbulent and radiative transfer of that cooling upwards. The locally colder air near the surface relative to the same height in the free atmosphere creates a pressure gradient sufficient to cause downslope flow, $u$. The greatest temperature deficit and therefore largest pressure gradient is located at the immediate surface.The strongest flow, however, exists some height above ground level due to surface friction retarding the flow magnitude near the surface, causing the idealized jet structure. The combination of surface friction and an ever reducing temperature deficit (and pressure gradient) with height produces a jet maximum within the middle third of the inversion depth.

The reader should note that katabatic flow is a much different phenomena than the nocturnal low-level jet found on the Great Plains and elsewhere around the world. The nocturnal low-level jet is caused by a different mechanism, has its supergeostrophic jet maximum located at the top of the nocturnal inversion or higher and typically attains higher speeds 


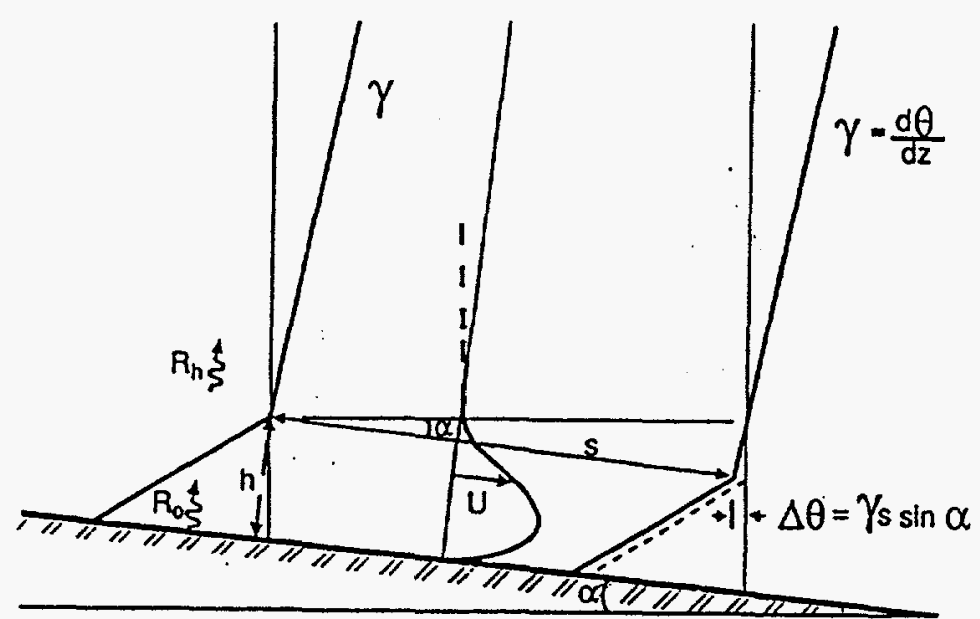

Figure 1.1 The conceptual model of katabatic slope flow of Manins (1992). The inversion is drawn for two different slope locations separated by a distance, s. $\alpha$ is the slope angle, $h$ is the inversion depth, $\gamma$ is the lapse rate of potential temperature, $u$, is the downslope wind speed. $R_{h}$ and $R_{o}$ are the longwave radiative transfer rates of the ground and air aloft, respectively.

than katabatic flow (Blackadar 1957, Bonner 1968, 1970, Fast 1990, Zhong et al. 1995).

Whereas Figure 1.1 is applicable to steady, undisturbed katabatic flow, the observations of katabatic flow in its natural state shown in Figure 1.2 indicate a generally unsteady nature. This plot was produced from ASCOT 1993 sodar observations at the head of Coal Creek Canyon near Boulder, Colorado along the Front Range of the Rocky Mountains. The overnight period from 1200 LST 2 October to 1200 LST 3 October is shown representing one of the most quiescent katabatic flow nights found between August and December, 1993. The upper panel shows horizontal wind speed, the middle panel wind direction and the lower panel vertical motion. All panels indicate a variable time structure in the depth and magnitude of katabatic flow. If one chooses an instant in time during the katabatic flow on the wind speed panel, a vertical jet profile similar to Figure 1.1 is found, but this profile constantly changes throughout the night. Coal Creek Canyon is aligned essentially east-west so that the transition from up-canyon to down-canyon flow near sunset (or vice-versa near sunrise) is shown where the wind direction contour lines change 
from dashed $\left(270^{\circ}\right)$ to dashed with the ${ }^{*}$ symbol (easterly flow). Note the extremely fast transition from down-canyon to up-canyon f low at the morning transition (0715 LST, within the 15 minute observation window). In panel c, solid contours show that upward vertical motion exists during the upslope phase and dashed contours indicate downward vertical motion during katabatic flow. On most other katabatic flow nights during ASCOT 1993, the flow was less steady and sometimes non-existent. It should also be noted that the

\section{Coal Creek Canyon Sodar 10/02-03/93}
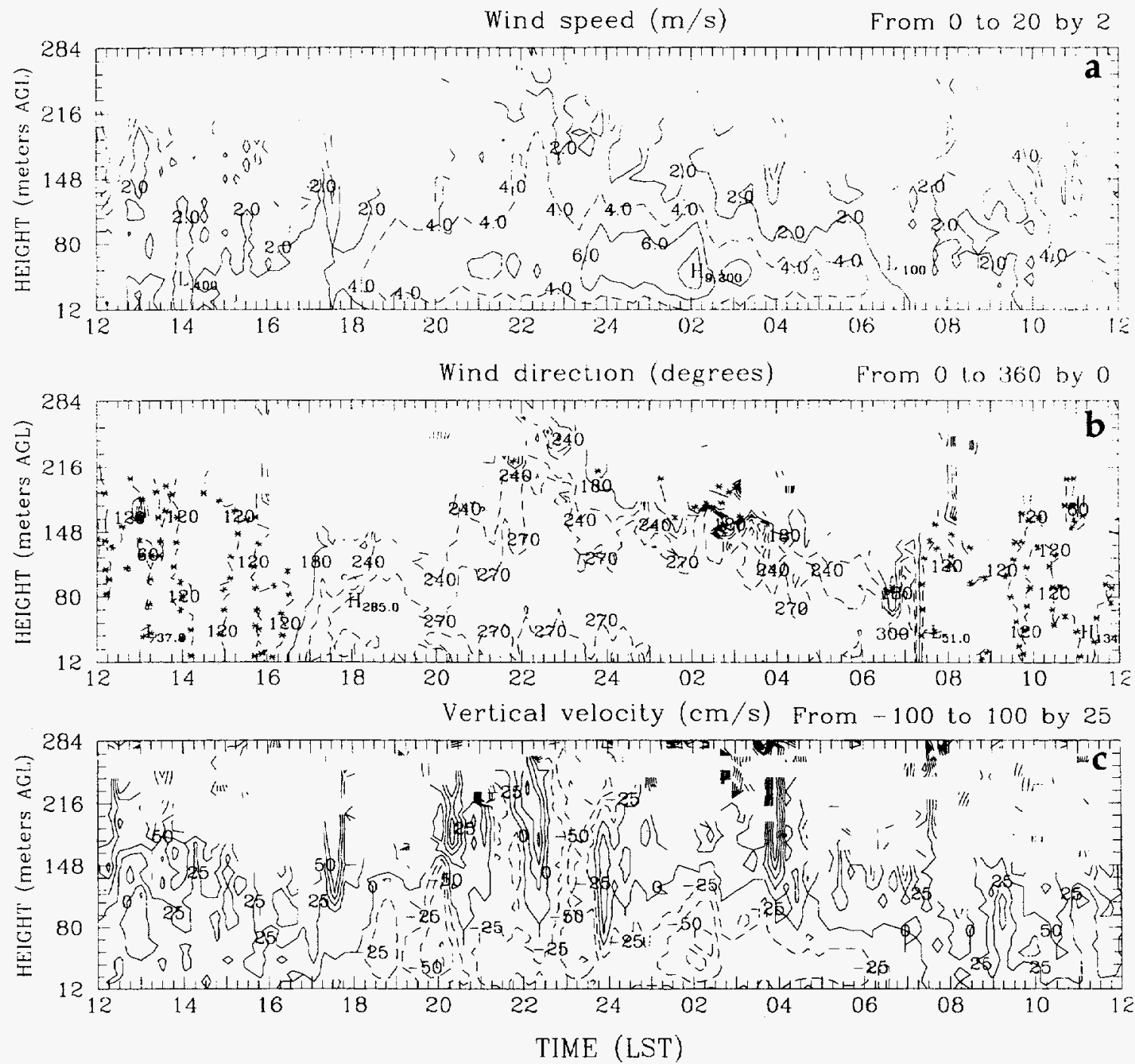

Figure 1.2 ASCOT time-height observations of katabatic flow at the head of Coal Creek Canyon on 02-03 October 1993 by sodar. Panels are a) horizontal wind speed and b) wind direction and c) vertical motion. The transition from anabatic to katabatic flow occurs before sunset over a period of less than 45 minutes (1615-1700 LST). 
physics governing pure slope (inclined plane) katabatic flow (see Figure 1.1) differs somewhat from that in a canyon where various slope flows converge to form down-canyon (or out-canyon if a level valley floor) katabatic flow. The dynamical differences between valley and slope katabatic flow will be exposed in Chapter 2 .

\subsection{Mountain Waves}

Mountain wave, or topographically-induced internal gravity wave, is the name given to the generic phenomena of an airflow in a stable atmospheric environment being diverted away from its initially horizontal, barrier perpendicular path by a barrier of some form.

The reaction of the airflow to the barrier changes with velocity, stability and barrier configuration. A large body of theoretical or otherwise idealized literature solving various
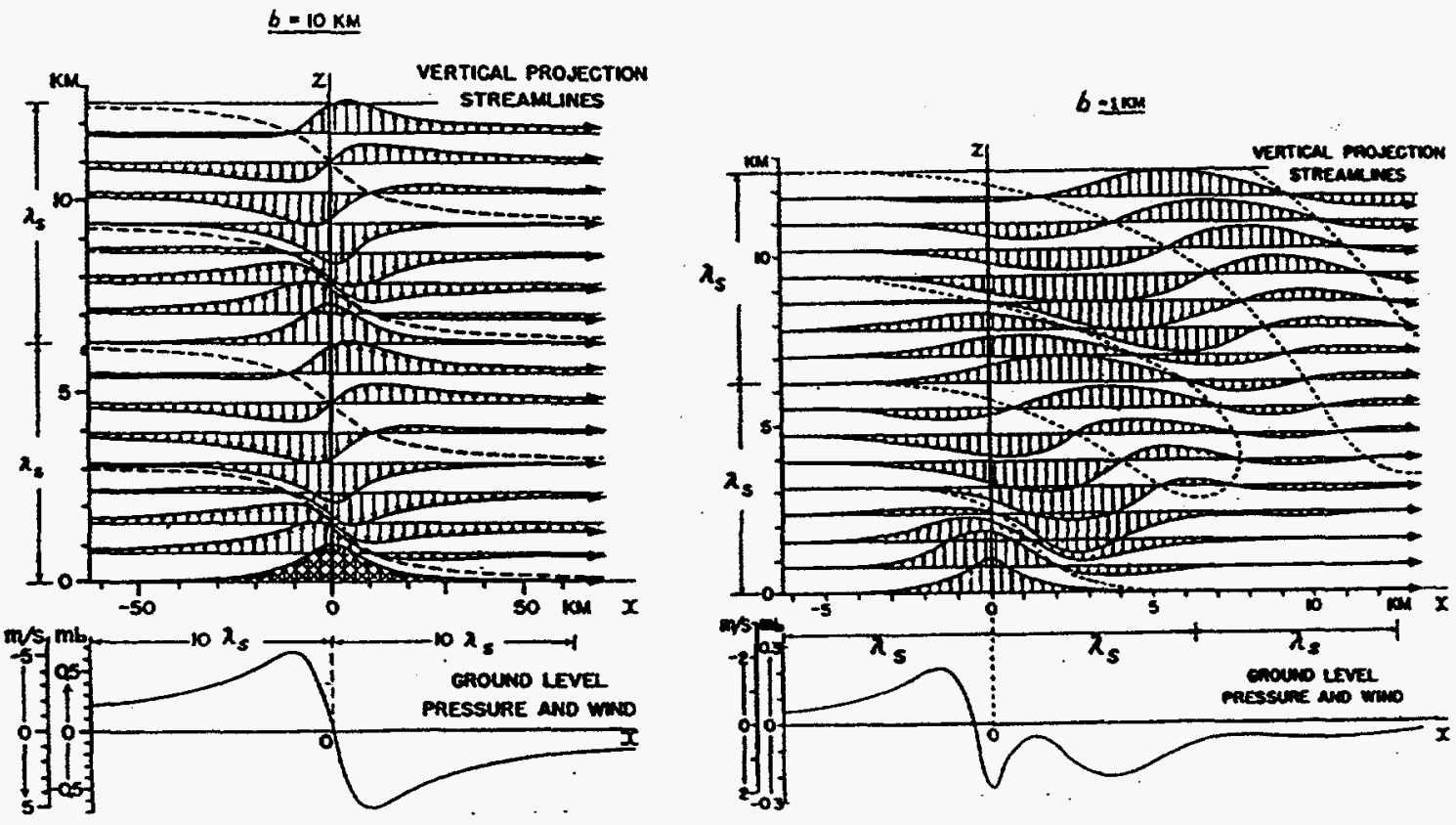

Figure 1.3 Idealized streamlines, surface pressure perturbation and wind speed perturbation from linear theory of mountain wave flow from Queney (1948). In this case the mountain is 1 $\mathrm{km}$ high, has a half-width of $10 \mathrm{~km}$ and $\mathrm{L}_{\mathrm{s}}$ is the mountain wave vertical wavelength. 
equation sets for certain parameter spaces exists, as well as a smaller body of observational studies. As in the katabatic flow case, observations are much less ideal than the theory. Figure 1.3 shows an example of a linear mountain wave flow solution from Queney (1948) where the initial flow is $10 \mathrm{~m} \mathrm{~s}^{-1}$ and the barrier width is 10 times larger than the wave perturbation. For this steady-state case, an upstream, mountain-induced, high pressure of $0.5 \mathrm{mb}$ is formed along the upwind side of the barrier which opposes the incoming westerly flow reducing it by $5 \mathrm{~m} \mathrm{~s}^{-1}$ In the barrier lee the opposite occurs causing an acceleration of the initial flow to $15 \mathrm{~m} \mathrm{~s}^{-1}$. The contortion of the streamlines indicates the path that air follows surmounting this barrier. Upstream flow and atmospheric stability are considered to be constant. In nature, of course, these simple assumptions are not met and topography is not uniform. Mountain waves are discussed in greater depth in Chapter 2.

Figure 1.4 from Lilly (1978) is indicative of what a realistic mountain wave case can look like. This figure represents a day where a severe windstorm, caused by non-linear interactions between the mountain wave and atmospheric stable layers, occurred over the Front Range of Colorado. Wind speeds approaching the Continental Divide exceeded 30 $\mathrm{m} \mathrm{s}^{-1}$ for hours at a time allowing for surface wind speed recordings in excess of $50 \mathrm{~m} \mathrm{~s}^{-1}$ near Boulder. Numerous severe turbulence reports and wind damage were reported and conditions were extremely unsteady. Whereas this is an extreme case, the vast majority of realistic cases, albeit much more benign, still have important effects on Front Range wind fields and turbulence.

\subsection{HYPOTHESIS}

Based on the unsteadiness of katabatic flows and mountain waves in their natural state and their frequent coincident occurrence, it is hypothesized that the variation of the near- 


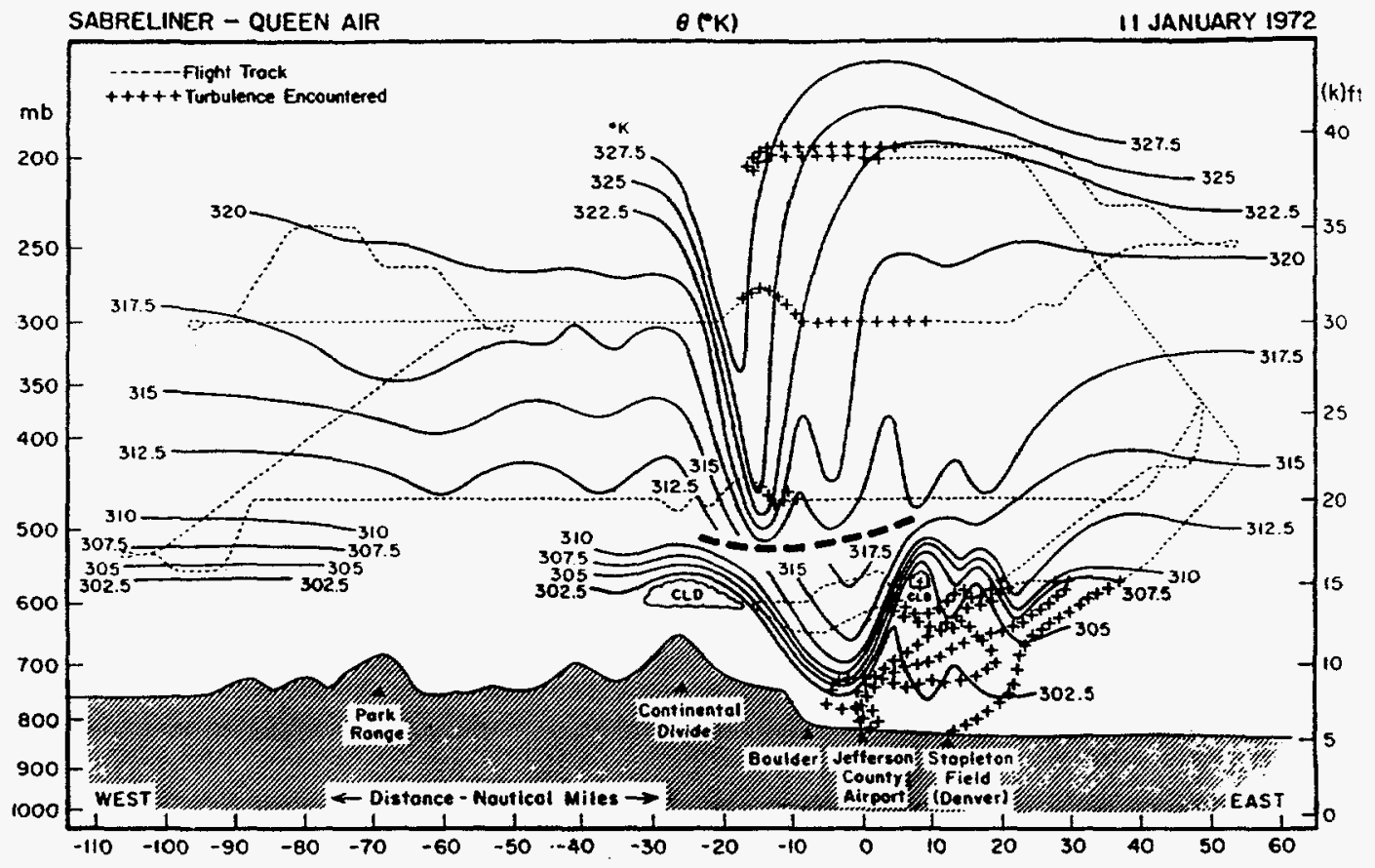

Figure 1.4 Observations of potential temperature structure (solid lines), cloud and significant turbulence $(+)$ during a severe mountain-wave induced wind storm along the Front Range of the Rocky Mountains 11 January 1972 from Lilly (1978).

surface, primarily radiatively-driven, nocturnal winds along the east side of the Rocky

Mountains can be partly explained by interactions with topographically forced mountain waves. This interaction may be manifested as shallower or deeper katabatic layers, stronger or weaker jet speeds, occasional or complete absences of katabatic flow, local gravity wave generation, increased variance of atmospheric fields or an alteration of layer stability. In turn, the amplitude and wavelength of the mountain waves themselves is partly determined by the evolving atmospheric stability in which both phenomena reside, resulting in a complex, highly interactive overnight evolution of atmospheric conditions.

\subsection{APPROACH}

This work will consist of three main parts, 1) literature review (Chapter 2), 2) observa- 
tional development (3) and 3) modeling investigations (Chapters 5-7), where each step contributes to formulating a comprehensive understanding of mountain wave/katabatic flow interaction (MKI). The literature review will focus primarily on katabatic flows, whose variability is being investigated, and studies relevant to the problem at hand. Some analytical models of katabatic flow will be discussed as well as the likely effect of mountain waves on near surface flows, such as katabatic winds. The observational development portion analyzes a number of katabatic flow case nights, from the ASCOT 1993 field program to examine the potential influence of overlying mountain waves. Katabatic flow nights are ranked using atmospheric measurements in the region of the canyons (a sodar and a few towers in Coal Creek and three sodars, two wind profilers with RASS and some towers at Eldorado, along with larger scale information) from the ASCOT 1993 experiment. Upstream soundings (represented by the Grand Junction sounding at $00 \mathrm{Z}$ and $12 \mathrm{Z}$ ) are used to develop a local Froude number climatology. This climatology is a baseline upon which a typical MKI case night is selected.

Using the information from the literature search and analysis of observations as a guide, a directed modeling effort commences. 2-dimensional simulations are used for model validation exercises, analysis of MKI and to guide 3-dimensional simulations. A limited set of systematic idealized 2 - and 3 -d sensitivity simulations over a $2.5^{\circ}$ slope, using various Froude numbers (including the zero wind case) with realistic nighttime radiative cooling are completed to attempt to isolate the effects of an overlying mountain wave on katabatic flow. 3-d modeling ends with a case study simulation. After a reasonably in-depth verification of accuracy by comparison with ASCOT observations, the case study is analyzed for the forcings at work using time series plots of difference fields, vertical integrals model 
tendencies. A final comparison of the modeling results to expectations from previous work and observational analysis combines enough information to develop a more complete understanding of MKI, with due respect to the other forces of influence.

\subsection{EXPECTATIONS}

This work extends our understanding of how two mountain phenomena (topographically forced internal waves and thermally forced drainage flows) interact. Enhanced understanding of the sum of these two influences on canyon winds contributes to, 1) local and canyon wind prediction, 2) the understanding of causes of wind variability along mountain ranges, 3) forecasting when ambient flow aloft will descend to the surface, 4) the understanding of lee side layering of wind and temperature fields and 5) the improvement of diffusion estimates for the thermally stable case dispersion models. This information is useful to local forecasters, the aviation (turbulence and shear) community, the agricultural community (frost protection) and the emergency preparedness/dispersion prediction community. 


\section{CHAPTER 2}

\section{Previous Related WORK}

Whereas the written study of katabatic flows extends back to Fournet (1840), Saigey (1842) and Hann (1879) and the study of mountain waves to the early work of Rayleigh (1883), Kelvin (1886) and Koschmieder (1921), the study of the interaction of these two phenomena has a far shorter history. As stated in Chapter 1, this work primarily focuses on katabatic flows and how mountain waves influence their variability (mountain wavekatabatic flow interaction or $\mathrm{MKI}$ ). This review therefore emphasizes an understanding of the historical work on katabatic flows and the more recent work on causes of variation in such flows. Other reviews of slope and valley flows can be found in Geiger (1975), Atkinson (1981), Barry (1992), Defant (1951), Mahrt (1982), Sturman (1987), Thyer (1965), Vergeiner and Dreiseitl (1986) and Whiteman (1990). The most thorough analysis and interpretation of the first century (1800's through 1945) of katabatic flow investigation can be found in Hawkes (1947). As one of the external forces causing variability in katabatic flows, mountain waves, in the following review, are discussed for what aspects of these waves are most likely to influence the structure of katabatic flow - an entire historical review of mountain waves is not attempted. Reviews of mountain wave phenomena and theory can be found in Queney (1948, 1960), Scorer (1972), Smith (1979), Baines (1987) and Durran (1990). This chapter ends with a review of work directly related to the investi- 
gation of MKI.

\subsection{KaTABATIC FLOW DYNAMICS}

The development of theory describing katabatic slope and valley winds has a long history whose origins begin in the early 1800 's. The description of this development by Hawkes (1947) is the most enlightening comprehensive text on this early work. Perhaps the most surprising conclusion is that Fournet (1840), in the first known scientific paper on katabatic flows (although Hawkes (1947) indicates that Fournet knew of Saigey's unpublished work from the 1930 's), correctly conceptualized how katabatic slope flows operate. The following description presents the dynamics of katabatic slope and valley winds, respectively, as they are known to function today.

From its Greek origins the term katabatic, where 'kata' means downward and 'batos' means moving beyond, can be interpreted to refer to essentially any flow that moves downward. This broad interpretation is inconsistent with the general use of the word, however, which typically refers to winds that flow down the topographic gradient or out of a valley due to surface cooling which gives this air a greater density than the free atmospheric air. This surface cooling normally is due to a net negative surface radiative balance but the cooling can be aided by conduction from an ice or snow surface such as in the Antarctic (Mawson, 1915, Ball 1956, 1957, Rees 1991), on the Greenland ice sheet (Nansen 1890, Loewe 1935, Den Broeke et al. 1994) and on alpine glaciers (Tollner 1931, Ekhart 1934, Defant 1951, Hoinkes 1954). In a valley terrain configuration the katabatic flows on the slopes that comprise the valley coagulate in the valley base where they will continue their course out valley (or down valley if the valley is sloped). This katabatic flow out-valley is often called the mountain wind. Numerous authors have offered their opinions on 
which terminology is best used in various circumstances (Talman 1911, Jeffreys 1922 , Atkinson 1981, Barry 1992), but in the following the term katabatic flow or katabatic wind will be used to generally represent surface based flow caused by surface cooling on either a slope or in a valley, where the context (valley or slope) will be obvious from the topic at hand. The terms 'downslope wind' or 'downslope flow' and 'mountain wind' may also be used where the former two terms refer to katabatic flow on a slope only as opposed to the latter term which refers to katabatic flow out of a valley. Downslope flow or downslope wind in this context should not be confused with the same terminology used in the mountain wave literature to describe wind storms in the lee of a barrier. Note also that this discussion will not describe anabatic flow, the closely related daytime analogue to katabatic flow, that moves upslope or up valley due to surface heating.

\subsubsection{Of downslope flow}

A dynamical explanation for katabatic slope flow began in the scientific literature with two French researchers in the early/mid 1800's. Fournet's (1840) explanation was based on circulation induced by near slope cooling of the air (although he was trying to explain valley winds near the Rhone in France) and at approximately the same time, Saigey (1842) produced an alternate reasoning based on the expansion of air columns. Later, a somewhat more detailed version of Saigey's (1842) idea was presented by Hann (1879). Although Hann's (1879) analysis dominated thinking for 30-40 years, the circulation based concept of Fournet (1840) eventually became the accepted explanation for katabatic slope winds. The transition to the Fournet conceptual model was cemented by Wenger (1923), Wagner (1932a, 1932b, 1938) and Defant (1933), who recognized the applicability of the circula- 
tion theorems that had been developed (Kelvin 1869, Bjerknes 1902) to the problem.

Although the circulation theorem has been more frequently applied to such large scale problems as the general circulation (Bjerknes 1902), its underlying principles can be used to determine the circulation for any baroclinic system, including thermally forced flows. The equation appropriate to katabatic flow, the relative circulation theorem, does not contain the effect of the Earth's rotation and can be written (Dutton 1976),

$$
\frac{d C}{d t}=\int_{S} \eta \cdot\left(\frac{R}{p} \nabla p \times \nabla T\right) d \sigma
$$

where $C$ is the circulation, $\eta$ is the unit vector normal to the area being integrated over, $R$ is the gas constant for dry air $\left(287 \mathrm{~J} \mathrm{~kg}^{-1} \mathrm{~K}^{-1}\right), \nabla p$ is the gradient of pressure, $\nabla T$ is the gradient of temperature and $\sigma$ is a unit of area and the integral is taken about the surface area of the circulation. The direction of rotation implied by this theorem is determined by using the right hand rule on the cross product contained in the integral. To interpret this equation relative to katabatic flows it is useful to consider an example in which we draw the actual pressure-temperature solenoid generated as slope air cools. Figure 2.1 shows the pressure and temperature fields produced by RAMS during a numerical simulation of katabatic slope flow $\left(2.5^{\circ}\right.$ slope $)$. The gradients of pressure and temperature are denoted by the thick black arrows and the route about which the circulation is determined is denoted by the slopeside parallelogram. The slight perturbation toward higher pressure along the slope causes the gradient of pressure to deviate slightly from vertical downward, whereas the gradient of temperature is from cold slope air towards warm ambient air. Using the right hand rule we therefore see that the required circulation gives downslope flow with cold air sinking (warmer air replaces colder air). This simple and intuitively sat- 


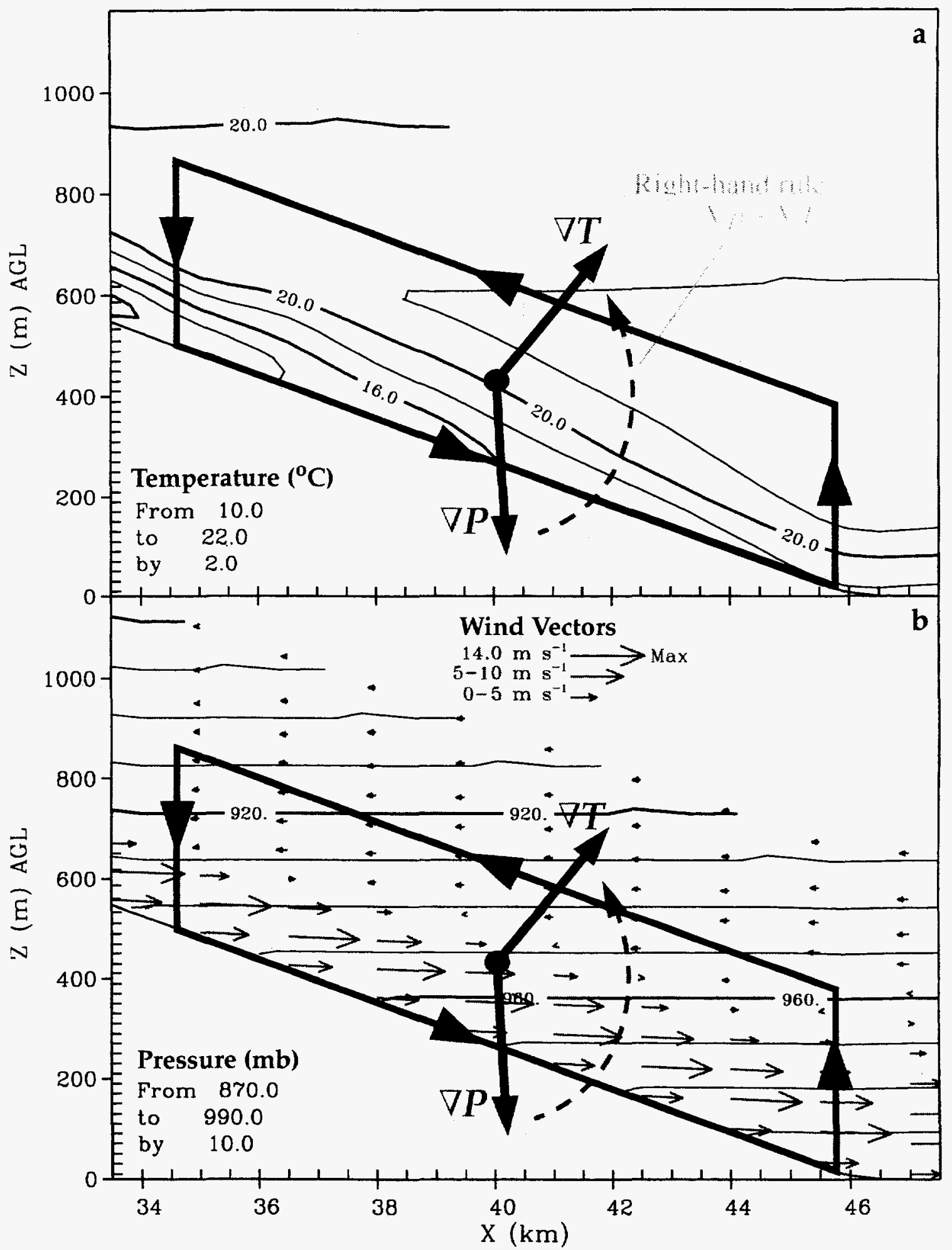

Figure $2.1 a$ ) temperature $\left({ }^{\circ} \mathrm{C}\right)$, and $b$ ) pressure $(\mathrm{mb})$ and wind vectors, from a numerical simulation of radiatively driven flow on a $2.5^{\circ}$ slope that show the application of Bjerknes' circulation theorem to describe katabatic slope winds. The thick parallelogram outlines the circuit evaluated and the heavy arrows depict the temperature and pressure gradients that require a clockwise circulation. Katabatic flow, with a jet and return flow, exists, as expected. 
isfying result explains katabatic slope flow. Indeed, as shown by the wind vectors in Figure $2.1 \mathrm{~b}$, the model reproduces a strong katabatic flow along the downslope portion of the circulation and return flow aloft.

The entire circulation and some measure of its intensity can be evaluated for this slope by putting the integral $(2.1)$ in the form,

$$
\frac{d C}{d t}=\oint R T d \ln p
$$

and evaluating around the circulation defined by the parallelogram shown in Figure 2.1. Using the values of $T$ and $p$ in the figure an estimate for the mean acceleration about the circuit due to the existing gradients is found to be approximately $0.006 \mathrm{~m} \mathrm{~s}^{-2}$. In 1 hour, without the decelerating effect of friction, such an acceleration would produce a wind speed of $21.6 \mathrm{~m} \mathrm{~s}^{-1}$ (which also presumes that the pressure-temperature solenoid could be effectively maintained by radiational cooling). Actual wind speeds in this numerical simulation were $14 \mathrm{~m} \mathrm{~s}^{-1}$ at near steady state.

Although the above analysis explains how the basic driving force of katabatic flows, buoyancy deficit, can induce a downslope katabatic wind, this flow can be categorized according to the actual force balance it has achieved at steady state. Such a classification was pursued by Mahrt (1982) in his well known work entitled, 'Momentum balance of gravity flows'. Using much of the terminology of the hydraulics community (as regularly used by Ball 1956) and the assumptions of no ambient flow and fixed temperature deficit he defined 4 types of slope flows, 1) shooting slope flow - where downslope buoyancy acceleration is offset by the decelerating forces of turbulence and advection; 2) equilibrium slope flow - where buoyancy force acceleration is balanced by turbulence; 3) Ekman- 
gravity slope flows - where the buoyancy forcing is balanced by coriolis and turbulence and 4) advective-gravity slope flow - where buoyancy is balanced by advection forcing. Although these classifications are not always applicable to real slope flows which are generally in constant adjustment, Mahrt (1982) uses these criteria to find that most previous observational work has observed either advective-gravity or shooting slope flows.

\subsubsection{Of down valley flow}

Since valleys themselves are composed of the slopes that define them and the valley floor, it is clear that the dynamics of slope flow will influence the dynamics of out-valley (or down-valley) katabatic flows. Numerous authors have discussed the dynamics of these winds both conceptually and in relation to the previous section's circulations theorem (see Wagner 1938, Defant 1951, Whiteman 1990, Barry 1992) and the following will simply summarize their descriptions. The interplay between the valley side slope winds, their associated cross valley circulations and the development of an out-valley wind can be simply represented as shown by Defant $(1949,1951$, see Figure 2.2$)$. Although drawn simply for this uniformly cooled valley, the flows shown in this schematic diagram are modified as the valley orientation, valley steepness and solar inclination angle change. Wagner (1938) describes the sequence of flow features overnight as follows:

"In the evening, when the downslope winds have begun, the upwards movement above the middle of the valley must be so great that it overcompensates for the downwards movement along the [valley] slopes. The result - provided the air is stably stratified - is more rapid cooling than above the plain, so that the temperature difference between valley and plain and simultaneously the pressure difference between plain and valley reach the zero value.

While cooling of the valley air due to vertical upwards movement above the middle of the valley progresses, the down-valley wind begins. As it gains strength, the air descending from the slope is conveyed to a progressively increasing degree out of the valley, so that progressively less remains for the air stream rising above the center of the valley, until ultimately - at the time of the temperature minimum of the valley air - the vertical movement above the middle of the 
valley ceases. When the downslope winds then cease in the morning, the valley air in its entire layer thickness parallel to the valley floor flows down the valley and thus heats up more rapidly than the plain.

It is immediately obvious that break up of the valley wind gradients after the slope winds have ceased occurs more slowly the smaller the slope of the valley floor. Thus it can be deduced that the phase delay of the valley winds relative to the slope winds is greater the less the gradient of the valley floor."

For completeness the entire diurnal cycle, as diagrammed by Defant (1949) is shown in

Figure 2.2. The development of the valley wind system is so complex and interactive that only few attempts (summarized in Egger 1990) have been made to analytically solve the valley wind problem. The majority of investigative resources into valley winds have been applied to observations or 3 dimensional modeling as outlined in parts of the following sections.

\subsection{THE NOCTURNAL BOUNDARY LAYER}

As much as katabatic slope flows have been investigated in their own right, the reason these flows form is because the cooled boundary layer they are a part of is located on a sloped surface. If, for the purposes of this discussion, we consider only the cooling nocturnal boundary layer, were it not for some amount of inclination the force causing katabatic flow would not exist. In the case of katabatic valley flows an inclined valley floor is not required as long as the valley sides are sloped, so that cool slope side air can drain into and fill the valley. Regardless of whether one is considering slope or valley katabatic flows, each requires an inclined surface somewhere within the system in question for their formation.

Since katabatic flows are an integral part of the nocturnal boundary layer it is reasonable to first consider the characteristics of the NBL itself before turning to the more complicated sloped NBL within which katabatic slope flows reside (Section 2.3). The flat or 


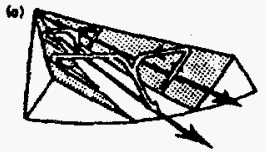

Floure 1: Air flow In the morning of sunrise. The upslope uinds begin (thlte arrows). The mountaln wind (bleck errous) is stlll present in the valley. The prossure drop is in the coun-welley direction. Tempereture: velloy is cold, plain is relotively werm. Changes in temperature luntll situation shown in Flgare 2): velley is boconing warmor, plein is becoming cooler.

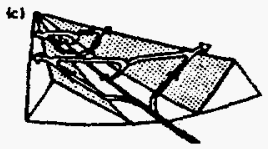

Floure 3: Alr flion from noon to urly atternocon. The. upslope winds ore decreasing. The villey wind is fully doweloped. The pressure droe is In the upvalley direction. Temperature: valley is vorin, plein is reletiveir cold. Changos in temporature cuntill the sltuetion storn In flgure 4): essentially none.

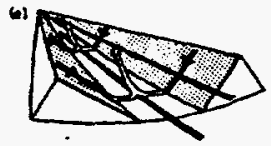

floure 5: Air llow in the owenling. The domslope vinds ore beginning. The velley wind is decreesing.

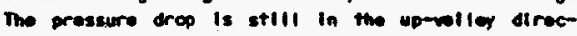
tion. Teepereture: It is slightly vanmer in the valfer then It is over the plain. Changes in the pereture (untll the sitwetion shom in flgure 6) the velley ts cooling reptdly: the plain is coolling only allghtiy.

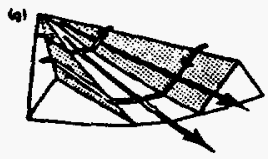

Flare 7: AIr flow st nlghte Mountaln viad is oresent olong with the downslope vinds. The pressure tros is In the com-relier direction. Tempereture: velley is cold, platn is roletively verm. Changes In Temperature (until the situation stom in flare B): velley is cooting; plole is cooling slightly tut is verm relotive to the velley.

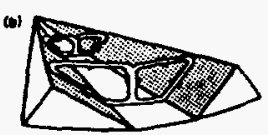

FIgure 2: Alr tiou in the forencon (about 9 em.). the upslope vinds are strong. The sreten is in. stote of transition tron mountain find to volley ulnd. Pressure drop: cero. Temperetures aquallzed. Chenges In temperature Cuntll the situstion shoun in Flgure 3): velloy is replaly bocoming wormer, tempereture over the plain is chenging slightly.

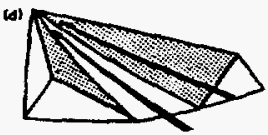

Flgure 4: Alt flow in tho late efternoon. The upstope winds hew died out. The strength of the velley wind is the sam os in Figure 3. The valley ulnd extends to the sldes of the slopes. The pressure drop is In the up-valley direction. Temperature: velloy is cerne plata is rele oly cold. Changes in tempereture (until the situstion shown in Figure 5 ): volley is slowly cooling.

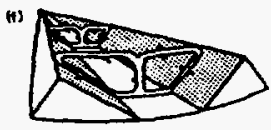

Figure 6: Alr flow froe late evening through the flest half of the night. Domslope vinds ore present. The syste is in a state of trensition from viliey wind to mountaln wind. Prossure drop: sero. Tempereture (untll the situation show In flgure I): willer continues to cool repldiy.

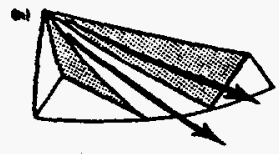

Figure s: Alr flow trom nlght untll wornlag. The dounglope ulnds have dled out. The counteln wind extends to the slaes of the slopes. The pressure crop is in the down-velley direction. Tempereture: cold in the velley, werwer over the platn. Cheapes in teo

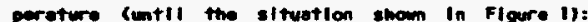
there ere only silght chenges in the veller end ower the plain.

Figure 2.2 The diurnal cycle of valley winds for clear sky, quiescent conditions as diagrammed by Defant (1949). See discussion in text. 
nearly flat NBL has begun to receive more attention in recent years because of the difficulty of accurately forecasting the variability of the nocturnal phase in large scale numerical atmospheric and dispersion models. Surprisingly the flat NBL contains a significant amount of variability in and of itself. Although this overview will focus on the general characteristics of the NBL for later comparison with the sloping NBL, for more extensive descriptions the reader is referred to Derbyshire (1995a,b) and Stull (1991).

\subsubsection{NBL Characteristics}

In general a 'boundary layer' in the atmosphere is the layer next to the surface affected by mass exchange processes caused by the surface. Within this definition the NBL is simply the nighttime expression of the response of overlying air to surface effects, and is most typically observed under synoptic high pressure (relatively undisturbed) atmospheric conditions. Figure 2.3 shows the diurnal cycle from convective or daytime boundary layer to the stable nocturnal boundary layer as depicted in Stull (1991) for quiescent conditions. A stable boundary layer will occur any time the air is cooled from below, such as by an ice, snow or relatively cold water surface, but the following short description will focus on the typical case where clear skies and light winds allow radiatively induced cooling.

The depth of a typical NBL in calm conditions is defined by the depth of its surface based inversion or increase of temperature with height. The inversion is caused by stronger longwave radiational cooling of the ground than the air above it, known as radiative flux divergence. This cooling can be as high as $4^{\circ} \mathrm{Chr}^{-1}$ very close to the surface but diminishes quickly with height. After sunset, without the heating from insolation the air adjacent to the ground surface quickly cools to the atmospheric temperature and soon is colder than the atmosphere above it, creating the inversion condition. Assuming the atmo- 
spheric pressure at the surface has not changed appreciably during the cooling, near surface air becomes denser and conditions are stable (cold/dense air beneath warm/less dense air). Depending on the atmospheric conditions the stable nocturnal boundary layer can grow as deep as 500 meters with an overlying residual layer. The residual layer is the neutral or nearly neutral layer remaining from the previous day's CBL. Without any external influences the NBL will suppress any remnant turbulence from the daytime due to its strong stability.

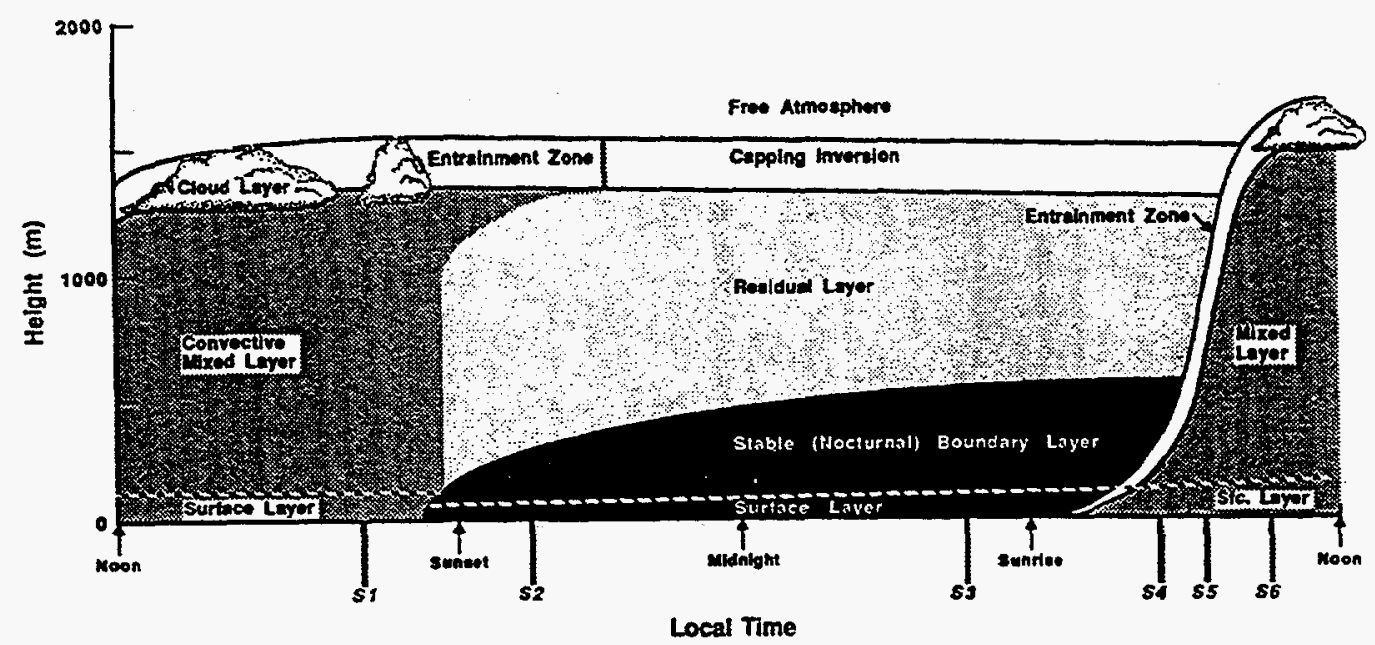

Figure 2.3 The diumal cycle of near surface conditions during clear sky, light wind conditions (Stull 1991). During the day a convective boundary layer grows and is forced by radiational warming. At night a more shallow stable boundary layer grows in response to surface radiational cooling.

\section{Variability}

Experiments have yet to observe an NBL completely free of external influence; more typically there are rather variable conditions that are difficult to predict. Data from aircraft flights over the approximately flat Great Plains during the Severe Environmental Storms and Mesoscale Experiment (SESAME) under clear skies and weak ambient flow provided the information for NBL studies by Mahrt (1985) and Ruscher and Mahrt (1989). Despite 
the relative flatness of this location weak katabatic flows did exist. The primary turbulent source in these cases was shear generation near the top of the inversion/katabatic layer. The corresponding downward heat flux warmed the upper half of the inversion but was balanced by radiational cooling and cold air advection, corresponding to the conceptual importance of that shear in the katabatic flow dynamical model suggested by Manins and Sawford $(1979 \mathrm{a}, \mathrm{b})$. The turbulence at inversion top often exhibited properties consistent with shear instability on scales of $300-400 \mathrm{~m}$. Interestingly, within the inversion the stratification flattened the larger scales of turbulence somewhat but at sufficiently small scales it was isotropic. These flattened turbulent structures are investigated in detail by Ruscher and Mahrt (1989) using principal component analysis. The NBL is found to contain quasitwo dimensional turbulence, fossil turbulence and vortical modes. Coherent structures in the NBL appear to occasionally collide causing convergence and a downward flux of heat from the inversion. Divergent zones appear to cause cold air below to mix upward into the ambient flow. The fluxes caused by these zones statistically explains a large portion of the variance in the nocturnal boundary layer observed during SESAME.

Statistically the nocturnal boundary layer has been found to contain pulsing or intermittent, changes of heat, velocity and turbulent characteristics as suggested by Ruscher and Mahrt's (1989) study above. Derbyshire (1995a,b) finds that the NBL variability is caused by a number of mechanisms including terrain, mesoscale forcing and gravity waves which may systematically prevent a model whose parameterizations are based on universal functions (such as RAMS) from matching observed quantities. Weber and Kurzeja (1991), during project STABLE (Stable Boundary Layer Experiment) at Savannah River Laboratory, measured 5-30 minute periods of turbulent bursts over horizontal distances of at least 
$30 \mathrm{~km}$ in the stable NBL overnight. Their measurements indicate that such episodes are preceded by near-surface cooling and a lowering of the local Brunt-Vaisala frequency. Continuously varying shear appeared to be responsible for the high variability in this NBL and significantly larger tracer dispersion than would be expected from standard stable atmosphere parameterizations.

\section{The low level jet}

Aside from the NBL jet within katabatic flow, quite often another sort of jet, somewhat ambiguously called the low-level jet (or LLJ) occurs most prominently overnight well above the ground surface. In course of my survey of studies of katabatic flows it has become clear that on occasion some authors refer to both katabatic flow jet and the lowlevel jet without clarifying the large differences between the two, or they are ambiguous about the jet type they have found (i.e. Devara et al. 1995). For example, Mahrt et al. (1979) use data from the Central Great Plains (O'Neill, Nebraska), Wangara, Australia and Haswell, Colorado (Western Great Plains) to discuss the nocturnal boundary layer. Whereas the jets observed at Wangara and O'Neill occur well above the surface at typical low-level jets heights, the jet observed at Haswell is more likely a surface based katabatic flow jet. Despite this difference, the characteristics of these phenomena are combined in one description of nocturnal jet behavior.

The dynamics of the LLJ have been discussed in great depth by a number of authors and this phenomena is dynamically quite different from katabatic flows. The explanations for the LLJ started most convincingly with Blackadar (1957) who described the LLJ as a feature that develops at or above the top of the nocturnal inversion due to the release of frictional coupling with the Earth's surface as the atmosphere stabilizes. Following this study 
were, Holton (1967) with another interpretation, Bonner (1968) with a climatology, Zeman (1979) with a LLJ parameterization (not suitable for katabatic flow parameterization), and Baughman (1981) with a discussion of LLJ formation in complex topography. More recently an extensive observational and numerical modeling study by Zhong et al. (1996) strongly supports Blackadar's (1957) theory in which inertial oscillation is the primary contributor to jet formation. Aside from the LLJ generally residing at a higher altitude (i.e. $500-800 \mathrm{~m}$ ) and maintaining higher wind speeds (i.e. up to $25 \mathrm{~m} \mathrm{~s}^{-1}$ on the Great Plains) than katabatic flow, the characteristic inertial acceleration to speeds greater than the local geostrophic wind speed delineates nocturnal LLJ's from nocturnal jets associated with surface cooling (see Figures 1.1 and 2.1).

\subsection{Katabatic Flow ObSERVATions}

\subsubsection{In valleys}

Katabatic flows were first observed in valleys because of the natural tendency for mankind to dwell in the base of valleys where water sources are readily available. Although knowledge of the diurnal nature of valley winds was known to the agricultural community for many years before, the first scientifically reported observations began in Europe near more populated valleys beginning with Fournet (1840) in the Savoie region of France and later in the Alps by Ekhart (1932a, 1932b, 1934), in Poona, India by Atmanathan (1931) and in England by Heywood (1933) among others. These observations made certain features of the katabatic winds obvious, 1) they develop based on surface cooling, 2) generally clear skies and quiescent synoptic conditions are most conducive to their development, 3) they are from tens of meters to a few hundred meters deep, 4) they form within a developing inversion, 5) their wind speed generally increases from the ground to 

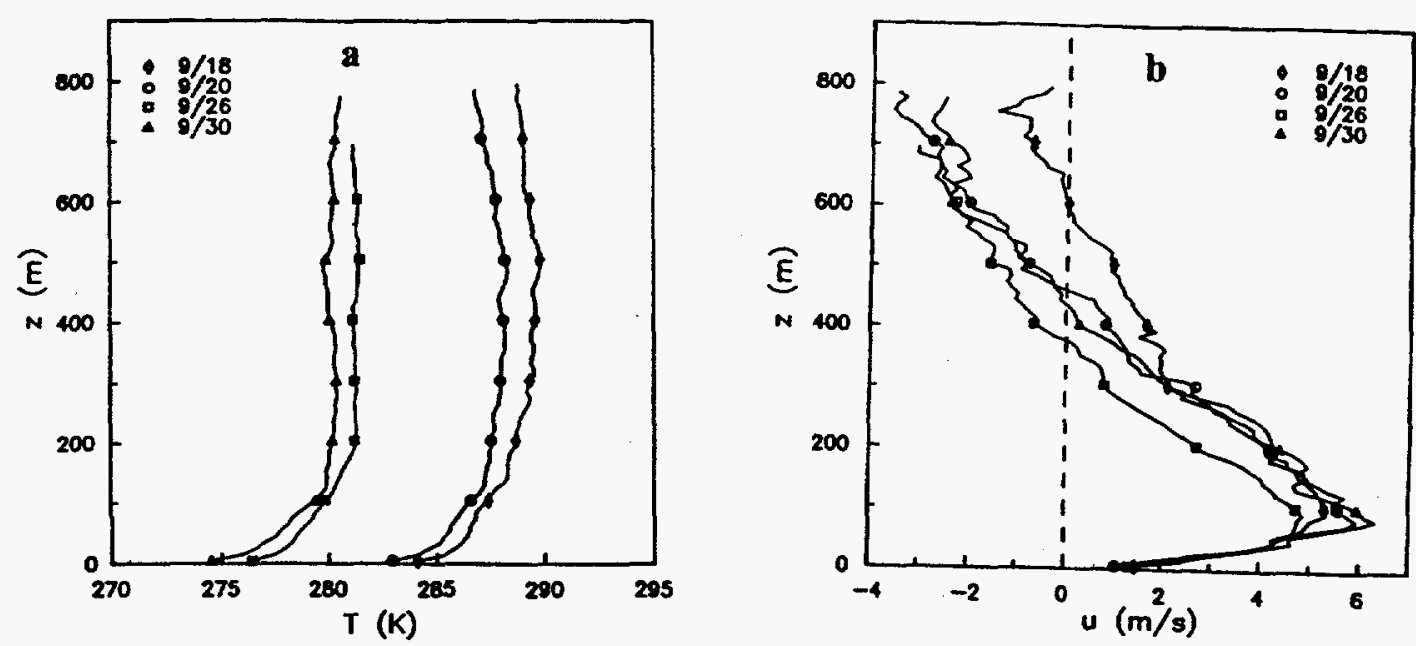

Figure 2.4 Observed mean (for 01-06 MST) vertical profiles of a) temperature (K) and b) wind speed $\left(\mathrm{m} \mathrm{s}^{-1}\right)$ in the down-valley direction of Brush Creek Valley, Colorado on four different nights. The valley ridgeline is at $600 \mathrm{~m}$ (Clements et al. 1989).

2-6 $\mathrm{m} \mathrm{s}^{-1}$ at some fraction of the inversion height and decreases from this jet to the top of the inversion and 6) they are common and frequent around the world.

Figure 2.4 shows the mean thermal and wind structure in Brush Creek Valley, Colorado as measured in katabatic valley flow during the 1984 ASCOT experiments (Clements et al. 1989). The temperature plot shows an obvious $7^{\circ} \mathrm{C}$ temperature inversion in the lowest levels $(300 \mathrm{~m}-400 \mathrm{~m})$ and an essentially isothermal structure above that to ridgeline. The valley is therefore entirely thermally stable on average. Clements et al. (1989) define the katabatic flow depth as approximately $400 \mathrm{~m}$ from the wind profiles, which corresponds rather well with the temperature inversion depth. The katabatic flow jet of $6 \mathrm{~m} \mathrm{~s}^{-1}$ lies at approximately $100 \mathrm{~m} \mathrm{AGL}$ or 0.25 of inversion depth, agreeing well with the early theoretical predictions of Prandtl $(1942,1952)$ discussed in Section 2.4. Davidson and Rao (1963) found this jet height to be $0.4-0.5$ of inversion depth in a valley near Manchester, Vermont and suggest that the ratio becomes smaller at steeper slope angles. Also evident 
in the Figure 2.4 is the negative flow above the katabatic flow - a so-called 'anti-wind', compensation current or return flow. This feature was routinely observed by Buettner and Thyer (1965) in a valley wind system near Mt. Rainier, Washington over 4 consecutive summer seasons which is consistent with the theoretical explanation of katabatic flow based on the circulation theorem (see Section 2.1). They find that the 'anti-wind' layer is the same depth as the down valley katabatic flow but weaker. Anti-winds are not always found in katabatic flow observational studies (Reiter et al. 1983) and for that reason the theoretical necessity of their existence is questioned (Ekhart 1932a, Davidson and Rao 1963). Most likely is that an anti-wind is simply difficult to observe in many cases because synoptic or regional scale influences generally dominate above the strongly forced near surface katabatic flow (Defant 1951). As obvious from the observations in Figure 2.4, the anti-wind can be observed in sufficiently quiescent conditions. Anti-winds are also likely to be difficult to observe in sinuous portions of a canyon.

The time evolution of valley katabatic flow has been well observed by a number of studies, but generalization is difficult due to different valley configurations, different observational locations and different measurement techniques. For instance, the measurements of Buettner and Thyer (1965) showed a katabatic flow peaking just before sunrise whereas Neff and King (1987) reported on a valley configuration where pooling in the lower valley slowed katabatic flow much before sunrise. Clearly, valley katabatic flow can peak in velocity at a variety of times relative to sunrise and sunset, depending on when the alongvalley pressure gradient is maximized at a particular location. Furthermore, McKee and O'Neal (1989) show that valley geometry can determine the local strength of along valley pressure gradient. Using the ratio of valley width to cross sectional area they find that wid- 
ening and narrowing of valleys along their course can strongly influence, and even dominate, the along-valley pressure gradient generated by radiative cooling. Davidson and Rao (1963) find that the flow in a smaller valley can be dominated by the valley wind system of a larger valley in which it resides which greatly influences the time of onset of the smaller valley's wind system. A similar effect is suggested by Defant (1951) for the Maloja wind in Switzerland where the wind in a valley blows in the same direction day and night, and was also observed by Buettner and Thyer (1965).

The transition from upslope flow to downslope flow near sunset has generally been found to occur quickly - over a period of less than 45 minutes (Defant 1951, Urfer-Henneberger 1967, Orgill and Schreck 1985, Vergeiner and Dreiseitl 1987), and the sodar observations discussed in Chapter 1 and Chapter 3 show transitions of 15-45 minutes. Buettner and Thyer (1965) found that the transition occurs at approximately the same time everywhere in the valley, about one hour after sunset and that both the slope winds and the katabatic valley winds begin simultaneously. However, this suggestion is contrary to their finding that a spiraling wind regime can develop near sunset as one slope is anabatic while the opposite slope is katabatic. Various other studies in different valleys have shown this transition to be prior to, at or well after sunset, suggesting that orientation, time of year and terrain shading also determine when the surface heat flux will reverse (Whiteman et al. 1989a, b). North-south oriented valleys, whose east side is last to be affected by insolation toward sunset, are likely to have down slope winds on the west side while the east side continues an upslope flow (Reiter et al. 1983). In this case it is also seen that the transition to the mountain wind from up valley flow will take at least as long as the along valley pressure gradient takes to reverse and, for a period, up valley anabatic winds can exist 
over the top of lower level down valley flow. Larger valleys have been found to develop along-valley pressure gradients that are more difficult to reverse than that of smaller valleys (Wagner 1938, Davidson and Rao 1963).

The transition from katabatic to anabatic valley wind can only occur as the along-valley pressure gradient is reversed by surface heating and the eventual destruction of the stable layers residing in the canyon. Numerous mechanisms to cause the destruction of valley inversions have been suggested as summarized in Whiteman and McKee (1982). Perhaps the most obvious mechanism is the flushing of the valley by ambient winds (Ayer 1961, Pollack 1924, Pielke 1985) where ambient flow aloft, perhaps altered by mountain wave dynamics, is strong enough and/or sheared enough to penetrate into the valley atmosphere and flush the valley. Whether anabatic flow would be observable in this high ambient wind state is dependent on the valley of interest. Whiteman and McKee (1982) focus on the more typical case of light ambient forcing such that surface heating is the primary mechanism of stable layer destruction. Figure 2.5 shows a schematic of this process. Surface heating of slopes and the valley floor generates near surface turbulence and upslope flow (not up valley flow necessarily as a down valley wind may still exist at this point). As mass is evacuated from the underside of the inversion it descends while also being partly eroded from the top. The process of mass evacuation and lowering continues until finally the inversion is broken. In winter, insolation may not be strong enough to break this inversion. More generally Vergeiner and Dreiseitl (1986) and Brehm and Freytag (1982) have found that an upper level inversion typically resides in the valley despite this heating which indicates that the valley inversion does not necessarily descend in all cases. Valley width and depth also play important roles in valley wind character. Buettner and 

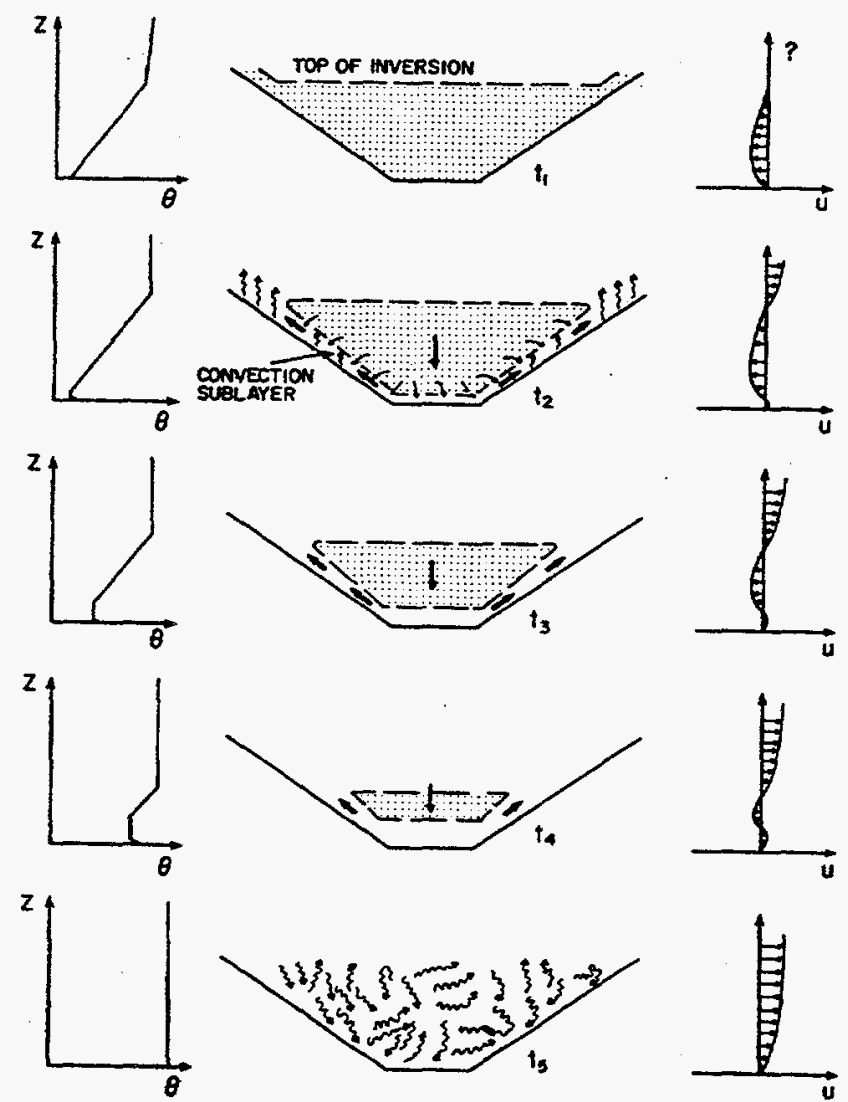

Figure 2.5 A schematic of the break up of a temperature inversion in a valley from Whiteman and McKee (1982). See text for details.

Thyer (1965) find that shallow valleys are rather easily influenced by overlying flow during the daytime making the observation of up-valley flows difficult, but the down-valley katabatic flow can still be well developed. Since anabatic flows are caused by surface heating, the turbulent eddies thus created during solar heating are conducive to the mixing downward of ambient flow and the masking of the anabatic mechanism. Banta and Cotton (1981) found that daytime mixing was a regular occurrence in North Park, Colorado, but that upslope flow did occur for a time prior to the mixing event. Toward evening, when the stabilization of the atmosphere prevented ambient flow mixing, katabatic flow was easily observed. McHattie (1968) found similar behavior in the Kananaskis Valley near Calgary, Canada. Even valleys so broad that the terrain appears nearly flat to humans, such as near 
Newark, New Jersey, have been found to be dominated climatologically by a katabatic flow regime (Davidson 1963b). For a deeper valley in Vermont, however, he finds that both the anabatic and katabatic flows largely control wind direction at Rutland Municipal Airport (although the anabatic portion was frequently influenced by overlying flow).

Davidson and Rao (1963) found that katabatic flow can develop even when the prevailing flow was $10-12 \mathrm{~m} \mathrm{~s}^{-1}$, whereas the anabatic flows were undetectable with similar flow aloft.

Although not explicitly ignored, the cross valley circulations crucial to down valley katabatic flow development, have not peaked the interest of nearly as many researchers as their down valley brethren. However, cross valley flows play an essential role in valley dynamics, particularly with respect to vertical motion, dispersion and thermal distribution (Wagner 1938, Tang 1976). Broder et al. (1981) observed that cross valley flow occurred as slope flows moved cross canyon toward the main valley flow. During katabatic flow this, by mass continuity arguments, leads to upward vertical motion and cooling in the valley center. They found that the cross valley component was important for determining the distribution and destruction rate of $\mathrm{O}_{3}$ in the valley atmosphere. Hennemuth and Schmidt (1985) found significant cross valley circulations throughout the course of a day in a small alpine valley that were crucial to the valley heat balance. Echoing this sentiment are Vergeiner et al. (1987) who find that without cross valley effects the up/down valley momentum budget requires vertical motions on the order of $0.10 \mathrm{~m} \mathrm{~s}^{-1}$. This rate of descent is required to replace lost mass in the valley center and corresponds to an adiabatic warming rate of $2.5 \mathrm{~K} \mathrm{hr}^{-1}$. This large value must then be balanced by radiative cooling and cold air advection to maintain the down valley flow as observed; they postulate that their neglect 
of cross valley flow caused an overestimate of the volume flux divergence and, by extension, the vertical motion at valley center. Adding a further complication Buettner and Thyer (1965) found they could measure no significant vertical component in the valley center despite conditions sufficiently quiescent for the observation of elusive return flow aloft.

\subsubsection{On slopes}

While katabatic valley winds are the most easily observed due to the amplifying effect of their confining topographic configuration, katabatic slope flows are a more basic dynamical element of valley katabatic flows. Despite the early observational emphasis on valley flows, theoretical development focused on steady state slope flows because of the complicating factor of three-dimensionality in valleys. Sufficient observations of natural slope flows themselves lagged behind the theoretical development, however, because of the difficulty of their observation. As you will see below, relative to the long history of katabatic flow interest, katabatic slope flow observations are a recent occurrence.

Perhaps the most well known of these studies is that of Manins and Sawford (1979b), who present a case study of katabatic flow in the Jeeralang Hills in southeast Australia Although not a perfectly formed slope (a slight valley shape, 1-2 km in width with ridges of less than $50 \mathrm{~m}$ ), the authors argue that this represents a typical slope in nature (Figure 2.6). The slope drops vertically $330 \mathrm{~m}$ at an inclination of $4.5^{\circ}$ from the horizontal with a roughness length of about $0.02 \mathrm{~m}$. The katabatic flow jet appears at 0.50 to 0.60 of inversion depth which is relatively high compared with typical katabatic flow measurements in valleys. Comparisons made by the authors with the one-dimensional theory of Petkovsek and Hocevar (1971) were unsuccessful because the simple theory requires an improper 


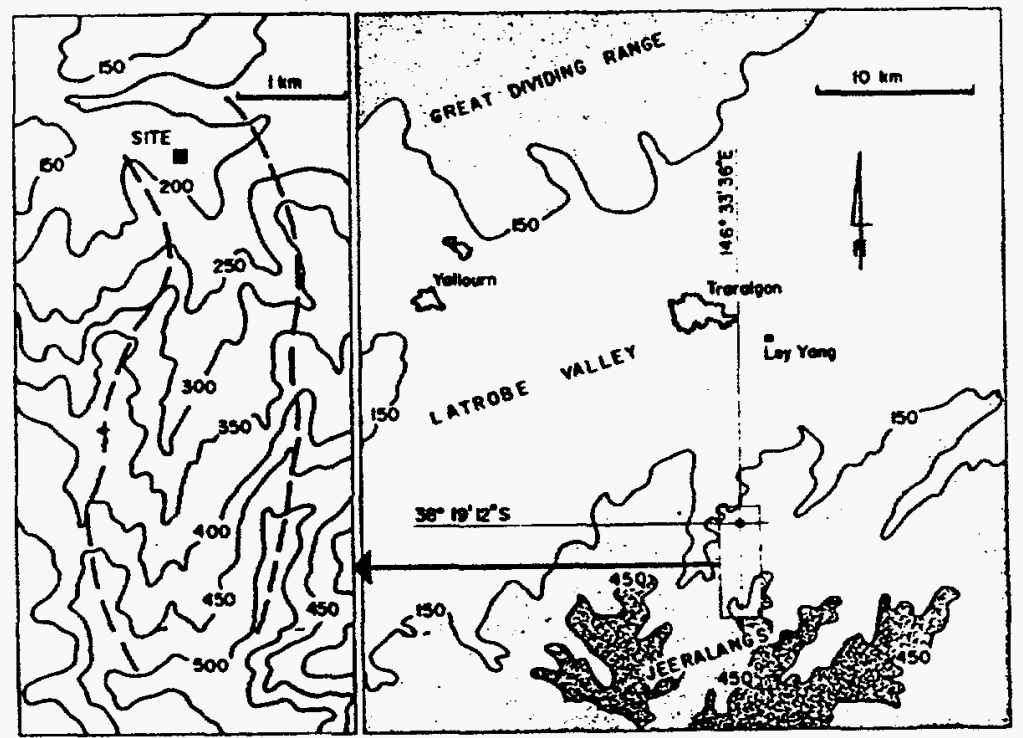

Figure 2.6 The terrain slope studied by Manins and Sawford (1979b). Only minor deviations from a flat slope exist.

amount of sensible heat flux and/or radiative flux divergence in the katabatic layer to account for the observed profiles. The authors argue that there must be some influence from side flows, i.e. three dimensional effects. Of course, they are considering features that are typical of valleys, not simple slopes, so perhaps a more ideal slope is necessary. An important conclusion they make is that the effects of turbulent mixing in the shear zone above the katabatic jet are more significant than those at the surface due to the strong stability at the surface. Their turbulent measurements are consistent with $R i \leq 0.25$ and a maximum in $\mathrm{Ri}$ at the katabatic flow jet. Mahrt (1979) corroborates high values of $\mathrm{Ri}$ at the jet due to a minimum in $\delta \mathrm{u} / \delta \mathrm{z}$ in that region.

Another series of well known slope flow papers were published by Doran and Horst (1983, Horst and Doran 1986, 1988) based on a series of observations over simple slopes and theoretical comparisons. Horst and Doran (1986) find that slope flow is generated consistently when the temperature deficit of the slope falls to a sufficiently low value as to 
overcome any existing ambient pressure gradient. They also conclude that for simple slopes the katabatic flow depth can be approximated as 0.05 of the distance downslope for slopes of $15-21^{\circ}$ which is supported by evidence from Clements and Nappo (1983).

Although this 'rule of thumb' does not seem to fit the observations of Manins and Sawford (1979b) above on a $4.5^{\circ}$ slope ( $80 \mathrm{~m}$ drainage flow depth, $330 \mathrm{~m}$ slope drop for a ratio of 0.24), Horst and Doran (1986) do find that their flows reasonably fit the model proposed by Manins and Sawford (1979a). The entrainment of air from above the katabatic flow is found to be consistent with the measurements of Ellison and Turner (1959) where entrainment rate is empirically derived depending on slope angle.

Manins (1992) used a rapid succession of tethered balloon soundings to calculate the importance of radiative flux divergence in the katabatic flow balance. He found that it is important to both the generation and retardation of the flow depending on location. Radiative flux divergence of up to $15^{\circ} \mathrm{C}_{\text {day }^{-1}}$ at the top of the katabatic flow layer where shear is great helps lessen the temperature gradient (by cooling the top of the inversion) and induce turbulence. The turbulence then can act to reduce the katabatic jet speed and inversion strength. Radiative flux divergence at the ground up to $20^{\circ} \mathrm{C}$ day ${ }^{-1}$ was a significant factor in the generation of katabatic flow. Being a very small scale feature, Manins suggests that no modeling study of katabatic flow has shown a significant of radiative flux divergence due to their generally poor vertical grid spacing and/or insufficient radiational parameterization. Some high resolution katabatic flow simulations in this work hope to alleviate this problem.

On slopes of less than $2^{\circ}$, Broeke et al. (1994) analyze observations on the Greenland Ice sheet in summer where the source of surface temperature reduction is the evaporative 
cooling over a melting glacier. This effect induces a consistent daytime katabatic flow of about $5 \mathrm{~m} \mathrm{~s}^{-1}$. Based on very complete heat and momentum budgets they find that radiative flux divergence is an important mechanism in the balance of these flows. In agreement with Manins (1992), radiative flux divergence is key for maintaining the katabatic flow temperature deficit as the descending flow would otherwise warm relative to the ambient stratification. Turbulent heat flux is also found to be a contributing factor in the cooling of the katabatic flow layer. Advection and the thermal wind term are found to be minor contributors to the total force balance of these flows at steady state. As is generally understood, buoyancy is the driving force for katabatic flow, and is balanced by friction and to a lesser extent, the coriolis force.

Another low slope angle $\left(2^{\circ}\right)$ study was conducted by Mahrt and Larsen (1982), on land that slopes toward the sea near Riso, Denmark. Without ambient flow the authors find that katabatic flow can continue to increase after initiation for a number of hours. Often the initial katabatic surge of cold air can overcome an opposing ambient wind. This surge is initially deep but later is shallow and more steady with occasional and smaller pulses. As the surge, regarded as a pressure head, proceeds downslope it slows and raises the opposing flow due to the adverse pressure gradient. The induced rising motion adiabatically cools the layer of ambient flow. Depending on the depth of this forced rising motion this mechanism has the potential to cool a significant portion of the overlying air.

\subsubsection{Of internal variability}

While the nocturnal boundary layer over relatively flat terrain has a certain degree of intermittency (e.g. Revelle 1993), in complex topography additional NBL variability has been observed. Katabatic flows, particularly those in valleys, are subject to numerous 
potential sources of variability aside from those evident in previous sections. Whereas the main purpose of this dissertation is to quantify the variability imparted to katabatic flows by an overlying mountain wave, a number of observational studies have identified other mechanisms that may induce katabatic flow changes. It is useful to be aware of these other sources of variability, and what kinds of variability are caused, in order to ascertain the significance of the proposed mountain wave mechanism. Whereas some sources of variability in katabatic flow are external (such as mountain waves and larger-scale pressure gradients), processes within katabatic flows themselves and their particular terrain configuration can also create variability. Tyson (1968) and Doran and Horst (1981) showed that this variability appears to create energy within the portion of the atmospheric energy spectrum associated with oscillations between 0.1 and 5 hours, thereby filling the spectral gap in atmospheric timescales observed by Panofsky and van der Hoven (1955) and van der Hoven (1957).

While not always easy to delineate from external influence, numerous studies in relatively quiescent atmospheric conditions (limited external influence) have noted katabatic flow variability on timescales of less than an hour. While offering no explanation, Buettner and Thyer (1965) observed fluctuations in katabatic flow speed $100 \mathrm{~m}$ above the valley floor from 1.5 to $6.5 \mathrm{~m} \mathrm{~s}^{-1}$ at 20-30 minute intervals, which are comparable to the $5-30$ minute episodes found by Weber and Kurzeja (1991). Manins and Sawford's (1979b) fluctuations in the 'skin-flow' (a few meters deep) were of the order 15 minutes. Start et al. (1975), during a tracer release (SF6) and oil fog photographic session in Huntington Canyon, Utah noted that pulsations in the katabatic flow appeared to be associated with tributary canyon interaction. Citing the Fleagle (1950) mechanism (oscillatory katabatic flow 
fluctuations during development) as a possible cause, Tyson (1968) found that cold air pulses occurred in approximately 1 hour intervals, and also found turbulent bursts with 20 minute intervals near Pietermaritzburg, South Africa. Observational statistical analysis by Doran and Horst (1981) showed fluctuations of about 1.5 hours. Nappo (1991) investigates the occurrence of sporadic breakdowns in the nocturnal stable boundary layer using observations of wind speed and temperature and their covariance. By band pass filtering at 5 and 30 minute intervals he extracts the deviation of the 5 minute structure from the 30 minute average structure. Based on the frequency of bursts (10-20 per night over both flat and complex terrain, more frequent over complex terrain) he speculates that a significant portion of the nocturnal fluxes are contained within these occasional bursts concurring with the conclusions of Ruscher and Mahrt (1989). Davidson and Rao (1963) also observed sporadic turbulent events as evidenced by sudden sub-jet mixing and above-jet mixing in shear zones.

Katabatic flows converging, accumulating or combining from different source regions can also cause self-induced variability. Erasmus et al. (1993) present observations near Greeley, Colorado of multiple scales of drainage flow interacting at that location (katabatic flows from the Cheyenne Ridge to the north, Poudre River to the west-northwest and South Platte River to the southwest). In more severe topography, Neff and King $(1987,1989)$ observe cold air accumulation in mountain valleys, elevated inversions, jets and complicated drainage flow structure due to the merging of numerous katabatic flows. As a katabatic slope flow descends into a more stable cold pool near the surface, variability can be produced by the resulting gravity waves. Gryning et al. (1985) document this effect for a coastal draining valley on the west coast of Greenland. In this study occasional 
surges of the valley floor cold air up the slope interact with the down flowing katabatic flow. The period of these surges, about 45 minutes, is consistent with the calculated period for gravity waves in this valley. Gravity waves in the stable surging valley bottom air were found to be amplified as they impacted the sudden change in slope angle (where they met the katabatic flow) much as ocean waves react when they encounter the shoreline (Stigebrandt 1976, Perkin and Lewis 1978).

While far less internally turbulent than their daytime counterparts (anabatic flows in the CBL), katabatic flows cannot often be idealized or characterized as mostly laminar, even in quiescent atmospheric conditions. In fact, Mahrt and Gamage (1987) dissect aircraft observations of stratified flow during SESAME and the Alps Experiment (ALPEX) and find intermittent turbulence driven by shear, continuous turbulence characterized by sharp boundaries, and weaker continuous turbulence. Stratification acts to decrease the slope of the spectral energy density to values smaller than predicted by inertial subrange theory. Weak continuous turbulence seemed to be decaying, perhaps as daytime eddies weakened. The primary generation of horizontal velocity fluctuations was in the direction of the shear, but observations taken in the mountains show longitudinal modes. Rees (1991) used cross-spectral analysis of observations in the nocturnal boundary layer to determine whether turbulence or gravity waves were primarily responsible for katabatic flow variability. He found that, for the Antarctic Ice Shelf, the level of gravity wave activity versus turbulent activity varied with prevailing meteorological conditions but gravity waves generally dominated in cases where $R_{i}>0.5$. Lower levels of the flow tended to be more turbulent consistent with greater surface drag.

Self-induced katabatic flow variability was also observed by Blumen (1983) as a cold 
drainage density current passed the Boulder Atmospheric Observatory on each of two consecutive days. Instabilities, probably gravity waves, set off by the passage of the density current are observed to alter the vertical motion field to heights at least as high as the highest observation at $300 \mathrm{~m}$ above ground level (AGL). Kelvin-Helmholtz waves are observed in the katabatic flow following the drainage front and, as the katabatic flow following the front continues past the $\mathrm{BAO}$, the gradual breakdown of the waves into turbulence - and then to less and less turbulence - was observed. These processes involve the entrainment of ambient air into the katabatic flow. The rate of entrainment may be most easily parameterized using the Richardson number (Richardson, 1920) because measurements of mean vertical motion are very difficult in such variable regimes (Blumen 1983).

Neff and King (1989), using sodar profiles and topographic information, investigated the source of variability in drainage wind profiles. Topographic constrictions and subsequent pooling, an effect detailed by McKee and O'Neal (1989), could elevate katabatic flows. Davidson (1961) concludes that turbulence in katabatic flows is highly sensitive to the position relative to local topographic features and the wind direction. The alternate narrowing and widening of a valley, its turns and merging with larger valleys, are found by McKee and O’Neal (1989) to possibly cause pressure gradients that oppose the down-valley katabatic flow gradient. Utilizing the fact that the cooling rate of a particular valley cross-section depends on its volume (the topographic amplification factor), they show that certain valley configurations, such as that where the ratio of valley width to valley area decreases with down-valley distance, should drain more effectively than others. A configuration where that ratio increases at some point along the valley is conducive to accumulation and pooling because of a greater cooling rate at that point than upstream. 
Consequently, a pressure gradient adverse to that conducive to drainage can be generated.

\subsubsection{Of external variability}

From the previous section we understand that katabatic flows, simply due to the environment they develop within can be far more variable than their generally idealized treatment. External meteorological influences, such as mesoscale and synoptic scale pressure gradients, wind fields, clouds (Tower 1903, Vergeiner and Dreiseitl 1987, Orgill et al. 1992) and temperature gradients are also of considerable importance to their evolution and variability. Within the sphere of these influences are mountain waves, whose effect on katabatic flow evolution and variability is the subject of this dissertation. The limited investigations of $\mathrm{MKI}$ are left to a more in depth, later review in Section 2.7.

As part of the ASCOT 1980 investigation near the Geysers area of Northern California Neff and King (1987) found that the depth of the Pacific marine inversion strongly modulated the initiation and evolution of drainage winds. They observed that buoyancy forces were able to overcome mesoscale and synoptic pressure gradients if topographic slope was steep enough $\left(5-6^{\circ}\right)$ and surface cooling large enough. Orgill et al. (1981) analyze the observations taken during ASCOT 1980 near the Geysers area of California in the Anderson Creek drainage. They find that warm surges occur on 1-4 hr time scales within drainage flow. These surges are inconclusively attached to oscillations of elevated inversions. Other surges may occur when the marine air penetrates inland in its diurnal oscillation. Orgill and Schreck (1985) extend the analysis to find cases where migrating mesoscale and synoptic systems disrupt the katabatic flow. Modified cool marine air was found to overcome the local katabatic flows regularly during a normal diurnal cycle. 


\subsubsection{Of dispersion}

The prediction of pollutant dispersion in the nocturnal boundary layer is complicated severely by its noted variability, so we expect observations of dispersion in katabatic flow conditions to corroborate unexpected variability. Davidson (1961) concludes that occasional turbulent activity in katabatic flow causes contaminants from sources located on the upwind side of a ridge to routinely be ventilated to the surface. Start et al. (1975) note that dispersion in katabatic flow is larger than would be predicted by flat terrain models in the high stability conditions present in the canyon flow due to three potential influences, ambient wind induced intrusions of turbulence, wake effects as flow intercepts in-canyon topographic constrictions and helical/ turbulent structures as tributary katabatic flow merges with main canyon flow. Indeed, the dispersion of pollutants in the high stability classes of the Pasquill classification is severely limited and, based on the above observations of true katabatic flow, probably incorrect in many cases (except the most laminar). Observations of tracer dispersion from the ASCOT 1991 experiment near Rocky Flats, Colorado (Shearer 1993) also indicated a greater variability than would be expected from typical dispersion parameterizations for the overnight stable case. Three-dimensional modeling of some of these case nights by Poulos and Bossert (1995) using RAMS and its Lagrangian Particle Dispersion Model (LPDM) verified the inadequacy of the dispersion parameterizations for stable cases. Some of the inaccuracies of the dispersion simulations were also undoubtedly due to imperfections in the RAMS model forecast of surface conditions.

\subsection{ANAlytical SOlutions for Katabatic Flow}

Prior to the development of computers, researchers could not simply integrate the equa- 
tions of motion to understand katabatic flow, so they sought to solve the problem analytically. Naturally this methodology required a sufficient simplification of the governing equations to allow a mathematical solution. The first analytical method solves for the vertical structure of the flow (e.g. Jeffreys 1922) but relies on given values of the exchange coefficients to determine flow characteristics. The second method eliminates the reliance on exchange coefficients, but must solve for layer average quantities only (e.g. Defant 1933). This 'bulk' approach to a fluid flow problem is often called the hydraulic method (Manins and Sawford 1979a). Although inevitably inapplicable to many real world cases due their simplifications, numerous interesting insights into katabatic flow behavior have been made using both techniques.

\subsubsection{Formulations with vertical dependence}

The first work of this type (Jeffreys 1922) has perhaps been quoted more often for its classification of winds by type (i.e. geostrophic, cyclostrophic, Eulerian, antitriptic), than for its solution to the katabatic flow problem. It is of some surprise to read the first sentences of this paper,

"The present paper arose from a suggestion made by Sir Napier Shaw to the author concerning the desirability of a quantitative theory of the katabatic winds. When an attempt was made to construct one, the complexity of the problem was found to be so great that I decided to give an account of the physical principles involved in it before proceeding to the details of the solution, and to reserve the elaboration for a later paper. To describe the special features of a katabatic wind, however, necessarily involves contrasting them with those of other winds, and this in its turn involves a description of these winds; so that it soon became clear that a general account of the dynamics of winds was required."

What follows in his paper is essentially a scale analysis and classification of all the winds of the Earth from planetary to local scale - quite an achievement for a work motivated by katabatic flow! However, at the end of his paper, Jeffreys (1922) returns to an analytical 
solution to katabatic winds (called 'antitriptic' winds). Using a two dimensional coordinate system where the vertical axis, $\mathrm{z}$, is perpendicular to the slope $\alpha$ and the horizontal axis, $\mathrm{x}$, is aligned with the slope, the simplified governing equations of horizontal motion, hydrostatics, anelastic continuity, and heat, respectively, are,

$$
\begin{gathered}
0=-g \cos \alpha-\frac{1}{\rho} \frac{\partial p}{\partial x}+K \frac{\partial^{2} u}{\partial z^{2}} \\
\sin \alpha \frac{\partial p}{\partial x}+\cos \alpha \frac{\partial p}{\partial z}=-g \rho \\
\frac{\partial}{\partial x}(\rho u)+\frac{\partial}{\partial z}(\rho w)=0 \\
u \frac{\partial T}{\partial x}+w \frac{\partial T}{\partial z}=K \frac{\partial^{2} T}{\partial z^{2}}
\end{gathered}
$$

where the local time rate of change of $u, w, T$ and $\rho$, horizontal and vertical advection, coriolis and y components have been neglected with the steady state assumption. In these equations, $\mathrm{g}$ is the gravitational force, $\mathrm{K}$ is the eddy viscosity (assumed equal for heat and momentum), $\mathrm{p}$ is pressure, $\rho$ is density, $\mathrm{u}$ is along slope velocity, $\mathrm{w}$ is slope perpendicular velocity and $\mathrm{T}$ is temperature. The solution of these equations requires expansion about small departures from the initial state (then neglecting higher orders), substitution of a stream function formulation for continuity and the substitution of lapse rates for the derivative terms in (2.6). Doing so, and neglecting the second term of (2.4) in perturbation form leaves three linear differential equations with constant coefficients whose solution reduces to,

$$
K^{2} b^{6}+\lambda g \alpha_{a}(b \sin \alpha-a)^{2}=0
$$

where $\mathrm{a}$ and $\mathrm{b}$ are constants, $\alpha_{\mathrm{a}}$ is the coefficient of expansion of air equal to $0.00331 \mathrm{~K}^{-1}$ 
in Jeffrey's paper, $\lambda$ is the deviation of the temperature lapse rate from the adiabatic lapse rate (such that $\lambda$ of $0.004 \mathrm{~K} \mathrm{~m}^{-1}$ corresponds to a temperature lapse rate of $\sim 0.006 \mathrm{~K} \mathrm{~m}^{-1}$ ) and a small slope, $\alpha$, is assumed (allowing $\cos \alpha$ to be considered equal to 1). Through consideration of the appropriate boundary conditions for the real atmosphere the six roots of this equation can be reduced to the following equations for potential temperature perturbation and downslope wind speed with height, respectively (using $l=\sin \alpha$ ),

$$
\begin{gathered}
\theta^{\prime}=e^{a x}\left(B_{1} e^{-\Lambda} \cos \Lambda+B_{2} e^{-\Lambda} \sin \Lambda+B_{3} e^{\phi} \cos \Omega+B_{4} e^{\phi} \sin \Omega\right) \\
u=\frac{K c_{1}^{2}}{\lambda l} e^{(a x-\Lambda)}\left(B_{1} \sin \Lambda+B_{2} \cos \Lambda\right)
\end{gathered}
$$

where $\Lambda=\frac{1}{\sqrt{2}} c_{1} z, \phi=\frac{a z}{l}, \Omega=\frac{a^{3} z}{l^{3} c_{1}^{2}}$ and $c_{1}=\frac{\lambda g \alpha_{a} l^{2}}{K^{2}}$.

Considering boundary conditions we further find, $B_{1}-B_{2}=0.0028 \frac{c_{1}}{\lambda l}\left|B_{2}\right| B_{2}$ which, with values plugged in, can be solved as a quadratic equation. These complicated relations can only be solved with other approximations and consideration of what values are small contributors to the profile and can be neglected. In particular, if a $=0$ (effectively eliminating $x$ dependence) and since $B_{3}$ and $B_{4}$ are small, the equations are readily solved as:

$$
\begin{gathered}
\theta^{\prime}=B_{1} e^{-\Lambda} \cos \Lambda+B_{2} e^{-\Lambda} \sin \Lambda \\
u=\frac{k c_{1}^{2}}{\lambda l} e^{-\Lambda}\left(B_{1} \sin \Lambda+B_{2} \cos \Lambda\right)
\end{gathered}
$$

Noting that $(2.10)$ solved for $z=0$ represents the temperature perturbation of the ground surface and that this solution is $\theta^{\prime}=B_{1}$, allows us to choose the coefficient $B_{1}$. Choosing $\mathrm{B}_{1}=6.5^{\circ} \mathrm{C}, \mathrm{K}=5.0 \mathrm{~m}^{2} \mathrm{~s}^{-1}, \alpha=5.0^{\circ}, \lambda=0.002 \mathrm{~K} \mathrm{~m}^{-1}$, solving for $\mathrm{c}_{1}$ and $\mathrm{B}_{2}$, and plugging 


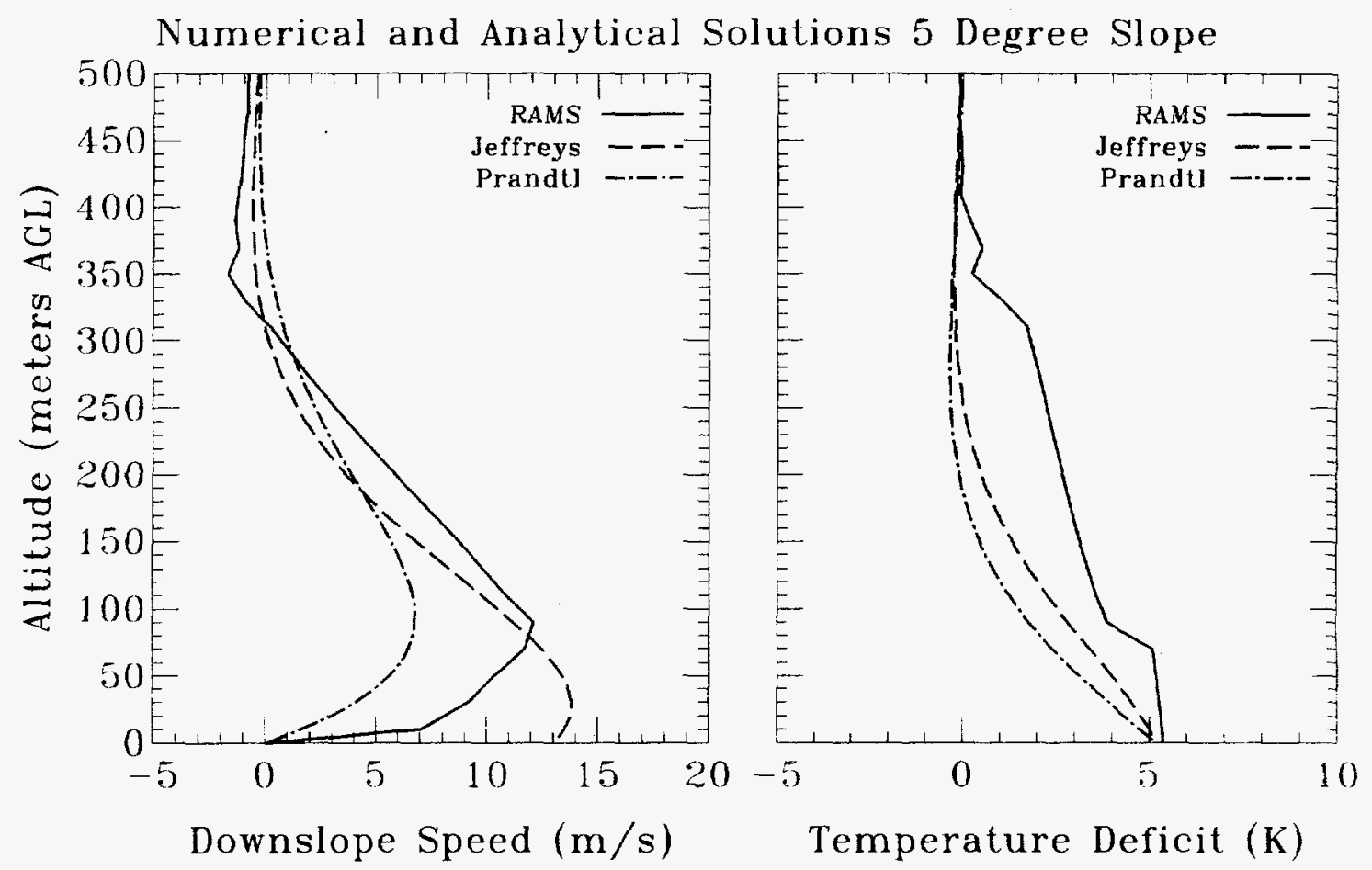

Figure 2.7 Numerical and analytical model solutions for katabatic flow down a $5^{\circ}$ slope. Each plot contains the solutions for the RAMS model, Jeffrey's (1922) analytical solution and Prandtl's (1942) analytical solution. Vertical profiles of a) downslope velocity and b) potential temperature deficit are shown.

in altitudes, $z$, gives the solution shown in Figure 2.7 with a dashed line. The surface velocities are too high compared with typical observations as also found by Jeffreys (1922) and a RAMS-generated numerical modeling result, perhaps indicating too little influence of surface friction in this analytical solution. Indeed, one of the criticisms of this type of steady-state result is that turbulent eddy diffusion does not change with altitude. Since frictional effects are included in this model it is somewhat of a surprise that no-slip boundary conditions were not applied to the solution (such a condition would force the lowest level wind speed to the more realistic value of $0.0 \mathrm{~m} \mathrm{~s}^{-1}$ ). The modeling simulation in Figure 2.7 is similar, except for using a steeper mountain slope, to those described in greater detail in Chapter 5 of this manuscript. This modeled vertical profile was extracted during nearly steady state, katabatic flow conditions approximately $8 \mathrm{~km}$ down a $10 \mathrm{~km}$ long, $5^{\circ}$ slope. The parameters chosen for use in Jeffrey's (1922) analytical formula were 
as close to those in the model simulation as possible. One can see that, despite the generally high surface flow value and subsequently low jet altitude, his formula reproduces many of the salient features of the flow. In particular, it captures the zero flow transition point near $320 \mathrm{~m}$ and the slightly negative flow above this point. As regards temperature deficit (see Figure 2.7b), Jeffrey's solution appears to contain too little 'mixing' of cold air upwards compared to the model simulation (solid line), or vice-versa. The numerical model tends to mix the regions just above and below jet height where shear is increased and therefore Richardson number is lowered. This 'K-dependent' behavior is not handled in Jeffrey's solution although the point where the modeled temperature deficit crosses zero is eventually reached.

Although little attention was paid to Jeffrey's (1922) analytical solution for katabatic flow, that of Prandtl (1942), who 20 years later also developed steady-state laminar equations for katabatic layer flow, has been given large amounts of attention. This discrepancy of attention may well be related to the relative simplicity of the Prandtl (1942) solution, as you will soon see below. In reality, however, each solution is fairly simple in its final form. The derivation of Prandtl's (1942) solution has been shown by a number of authors (i.e. Defant 1949, Rao and Snodgrass 1981) so it suffices to simply write down the result here. The equations for temperature perturbation and down-slope velocity with height, respectively, are:

$$
\theta^{\prime}(z)=\Delta \theta_{o} e^{\frac{-z}{l}} \cos \frac{z}{l}
$$




$$
u(z)=\Delta \theta_{o}\left(\frac{K_{H} g}{K_{M} \theta \frac{\partial \theta}{\partial z}}\right)^{0.5} e^{\frac{-z}{l}} \sin \frac{z}{l}
$$

where $\mathrm{z}$ is the height perpendicular to the slope, $\Delta \theta_{\mathrm{o}}$ is the prescribed surface potential temperature deficit, $\mathrm{g}$ is gravity, $\alpha$ is the slope angle, $\theta_{0}$ is the ambient potential temperature, the eddy diffusivity for heat, $\mathrm{K}_{\mathrm{H}}$, and momentum, $\mathrm{K}_{\mathrm{M}}$, are considered equal $\left(\mathrm{K}_{\mathrm{H}} / \mathrm{K}_{\mathrm{M}}\right.$ $=1)$ and $l$ is,

$$
l=\left(\frac{4 K_{H} K_{M}}{\frac{g}{\theta} \frac{\partial \theta}{\partial z}(\sin \alpha)^{2}}\right)^{0.25} .
$$

Calculations with (2.12)-(2.14) where only slope angle was varied showed that the jet speed is invariant with slope, although its altitude and katabatic layer depth shrink with increasing slope. Prandtl (1942) himself was somewhat surprised there is no dependence on slope angle in these equations (though contained in (2.14)), but explains that for ever smaller slopes the downslope component of buoyancy becomes smaller and that this is offset by reduced adiabatic warming relative to ambient stratification (presuming a slope of sufficient length to reach steady state exists). Of course, in a neutral atmosphere this is not the case, but Prandtl's solution is invalid when $\mathrm{d} \theta / \mathrm{dz}=0$ (in Jeffrey's 1922 solution $\mathrm{u}$ becomes trivially zero in the neutral case). The results of this solution are shown in Figure 2.7 with a dash-dot line for comparison with model results and Jeffrey's analytical solution. We see that Prandtl's solution produces a jet height and shape, jet speed and zero surface velocity more like the conceptual model of Manins (1992) shown in Figure 1.1. Its flow speed is too slow compared to the model result and the transition from downslope to upslope return flow occurs at too high an altitude. Similar to Jeffreys (1922), but slightly 
worse, the temperature deficit with height falls off more rapidly than the numerical model, yet eventually matches the height of zero temperature perturbation. No observational data of katabatic flow over sufficiently ideal topography with a $5^{\circ}$ slope exists for additional comparisons (Atkinson 1981 contains a list of katabatic slope flow observations).

The Prandtl (1942) solution has been verified with both modeled and observed downslope flow speeds. Defant (1949) compared flows along slopes near Innsbruck to the theory with reasonable success, although as in the above comparisons the theorized above-jet drop in flow speed is too high. Rao and Snodgrass (1981) find a similar inaccuracy in their modeling study, suggesting that the invariable eddy diffusivity in the theory prevents better comparison. Their comparison of vertical temperature deficit profiles indicates that the theory overpredicts at lower elevations and underpredicts aloft, unlike the comparison with RAMS above.

Modifications to the Prandtl (1942) theory have also been made. Defant (1949) included time variability in the theory and showed plausible figures of vertical structure in time. Gleeson (1953) included coriolis force, valley wall effects and a diurnal cycle of temperature in his extension from mere slopes to confined valleys. Lykosov and Gutman (1972) also included the coriolis force. Gutman $(1953,1972,1983)$ considers the basic theory in depth, and in 1983 investigates the external parameters that would affect the accuracy of Prandtl's (1942) theory, including a Monin-Obukhov surface layer treatment. His conclusions include a plea for detailed slope and valley flow observations. The possibility of the temperature perturbation varying along the slope in the Prandtl (1942) solution has been addressed by both Egger (1981) and Kondo (1984) for the daytime case.

The reader should also be aware at several attempts to analytically solve for the 3- 
dimensional circulation and temperature structure of a draining valley. Egger (1990) has summarized this work in great detail so further explanation will not be presented here, aside from a brief synopsis. The bounded, idealized valley heating problem solved by Gill (1966) reproduced the qualitative features of such flow as verified by laboratory observations (Elder 1965) and numerical simulations. Later, acting on an interest in the cross valley circulations required by valley flow, Vergeiner (1982) and Brehm (1986) pursued a theoretical route similar to Gill (1966). Brehm's result was primarily applied to up valley flow, however. Vergeiner (1987) produced a two dimensional analytical model for valley flow incorporating the effects of the third dimension, valley sides, via the topographic amplification factor. This model successfully represents the qualitative features of both up and down valley flow as a function of along valley distance.

\subsubsection{Layer average formulations}

The first application of the layer-average or bulk technique to katabatic flow was by $A$. Defant (1933) as reported by F. Defant (1949). To find an estimate of the average downslope flow in a katabatic layer, Defant (1933) considers the atmospheric to consist of twolayers - a katabatic layer of depth $\mathrm{H}$ at potential temperature $\theta_{2}$ underneath a free atmospheric layer at $\theta_{1}$. Considering the lower layer $\theta$ deficit and the associated horizontal pressure gradient, Defant (1933) finds that the mean katabatic layer flow, $O$, down a slope of angle $\alpha$, is

$$
O=\left(\frac{g H\left(\theta_{1}-\theta_{2}\right)}{\mu \theta_{1}} \sin \alpha\right)^{\frac{1}{2}}
$$

where $\mathrm{g}$ is gravity, and $\mu$ is a dimensionless constant representing the effect of friction. Using this formulation Defant (1933) was able to determine that katabatic flow should 
become unstable if the inclination of the slope becomes greater than $4 \mu$ agreeing with the results of Jeffreys (1925). Using Defant's suggested value for $\mu$ of 0.002 , it follows that katabatic flow on rougher slopes will be more steady than those on smoother slopes. For this value of $\mu$ the flow is unstable where $\tan ^{-1} \alpha>0.008$ or $\alpha>0.46^{\circ}$ exists; for the instability to form on this slope, however, requires a very long slope length (Ball, 1956). Relating $\mu$ to $z_{0}$ in this equation would be a useful adjustment towards applicability.

One of the early and more widely known bulk formulations is that of Fleagle (1950) which retains time dependence. Using horizontal coordinates as opposed to slope following, he analyzes the bulk flow characteristics for air cooling by contact with a radiatively cooling slope. In its initial stages the flow is found to vary periodically about a final equilibrium value. The equilibrium value is proportional to the net outgoing radiation and inversely proportional to cooling depth and slope. The latter relationship shows that for a given stratification, slower bulk wind speeds are expected on steeper slopes. His argument for why drainage flow speed decreases with increasing slope is that stronger adiabatic warming more effectively offsets the radiational cooling. In contrast, Prandtl's (1942) analysis shows no flow speed dependence on slope (see (2.12)-(2.14)) and calculations of the average over the Prandtl's katabatic layer are also slope independent. Fleagle's (1950) calculations also show that equilibrium is achieved more quickly as friction increases, which indirectly supports Defant 's (1933) result that rough slope surfaces are more steady than smooth slope surfaces.

A number of papers modified Fleagle's (1950) analytical result. Petkovsek and Hocevar (1971) extended the theory somewhat where their model of bulk katabatic flow features included net radiation, friction, slope angle and lapse rate as free parameters. In doing so 
they find more realistic results than Fleagle (1950) or Thyer (1966) when compared to observations due to the lapse rate parameter. They find that as lapse rate increases, slope decreases or friction decreases the time to equilibrium increases. Equilibrium velocity is proportional to cooling rate, independent of friction and inversely proportional to steepness. Comparisons by Streten et al. (1974) to Petkovsek and Hocevar's (1971) model were moderately successful. McNider (1982) also solved Fleagle's (1950) problem but added the effects of an ambient lapse rate finding that increasing the lapse rate decreased katabatic flow velocity.

In an application to the intense katabatic flows of the Antarctic ice sheet, Ball (1956) used the hydraulic approach without entrainment. Despite this approximation, his model was successfully able to reproduce many aspects of observed Antarctic katabatic flows (Ball 1957), including the strength of the observed pressure rise during katabatic flow lulls. The theory and observations suggest that the lulls and associated pressure rises are due to a hydraulic jump phenomena as shooting katabatic flows exceed a critical depth. As part of a physical modeling study (also described in Section 2.5.1), Ellison and Turner (1959) extended Ball's (1956) approach to include entrainment as dependent on Richardson number. Briggs (1979) further investigated Ellison and Turner's (1959) model and found he could predict drainage flow depth as approximately $5 \%$ of the vertical drop of the katabatic flow as also found by Doran and Horst (1983). Later, Briggs (1981) expanded the model to parameterize vegetative canopy effects in a bulk sense. The incorporation of canopy effects, compared to a model where roughness is prescribed as an attribute of the ground surface, was found to reduce drainage flow speed and volume while increasing cooling as a function of downslope distance. Comparisons with measurements from 
ASCOT's 1980 study near Anderson Creek, California were moderately successful.

Among the more well known recent hydraulic model extensions is that of Manins and Sawford (1979a) who subject a katabatic layer to cooling, surface stress and interfacial entrainment. They claim that katabatic flows are supercritical (or 'shooting flows') in most cases and, in some conflict with Jeffreys (1922), Prandtl (1942) and McNider (1982), that surface stress and ambient stratification are relatively unimportant. Their model uses a slope parallel coordinate system and assumes that the acceleration of flow is due to advection, pressure gradient, buoyancy. The hydrostatic approximation restricts this model to slopes of less than $10^{\circ}$ and most appropriately to those less than $5^{\circ}$ (Pielke 1984). The katabatic flow layer potential temperature is described as a balance between advection, turbulent diffusion and radiative flux divergence. The equations are then put in integral form following Ellison and Turner (1959) where entrainment is included through the continuity equation as a fraction of the downslope velocity and is further modified by Richardson number. Based on a successful comparison to bulk katabatic slope flow observations they conclude that ambient air mixing is responsible for 1) katabatic flow growth with distance downslope and 2) much of the retardation stress on the flow, as opposed to surface generated deceleration.

A more recent and quite revealing application of the hydraulic approach to katabatic flow can be found in van den Broeke et al. (1994, see also the observational description in Section 2.3.2). Here the application is to the katabatic flows of the Greenland ice sheet which form most strongly during summer when the underlying glacial surface is much cooler than surrounding ice free regions. One advantage of this location is the long slope (150 km of ice sloping to the sea) over which the katabatic flow has to reach equilibrium. 
Observational evidence indicates that in this case the advection term can be suitably ignored. Similar to Manins and Sawford (1979a) the temperature of the katabatic layer is found to be a balance between surface sensible heat flux and radiative flux divergence (where advection can be neglected due to equilibrium). Differently, though, they find that the lapse rate of potential temperature is important to the heat budget.

\subsection{KATABATIC FLOW MODELING}

The recognition that katabatic flows are caused by an increase in density of air due to cooling made them a natural candidate for exploration by the laboratory tank modeling community of the early 20 th century. By introducing a more dense fluid under a less dense fluid within the idealized conditions of the laboratory, researchers found that they could gain insight into the dynamics of these density currents. As always with physical modeling the question of applicability to the real atmosphere lingers, but the realistic aspects of the experimental results makes a sufficient argument for their consideration. Beyond physical modeling, with the advent of numerical modeling techniques their has been a large number of publications, ranging from idealized to realistic simulations of katabatic flows. In the following, an attempt is made to reconcile the findings of the numerical modeling community with that of their diligent observational co-workers of the past and present.

\subsubsection{Physical modeling}

In experimental fluid modeling tanks, the pursuit of information relevant to katabatic flows has been limited, but successful. In these studies the lower (upper) surface of a fluid tank is given a certain downward (upward) inclination to the horizontal and the tank is filled with a uniform density fluid (often water based). To initiate a flow similar to katabatic flow in some respects, a second fluid of greater (lesser) density is introduced at 
the upper (lower) portion of the slope.

This technique was utilized by Ellison and Turner (1959), in perhaps the classic paper on physical modeling of katabatic/gravity flows, where they released salinated water into a fresh water fluid tank. They find that entrainment coefficients are generally dependent on Richardson number and that the depth of flow is unchanged downslope for strong overlying stability. In weak overlying stability the flow deepens with distance downslope. Their velocity jet max appears in the lower portion of the inversion (around 0.3 ) as defined by density distribution, not far from the analytical prediction of 0.25 from Prandtl (1942). Britter and Linden (1980) in a similar experimental set up, analyze the entrainment and gravity head growth of a gravity current moving down slopes from $0-90^{\circ}$. Salt brine is introduced into a fresh water container at a regular rate as in Ellison and Turner (1959) emulating a dense flow into a neutral atmosphere. In this configuration, entrainment increases with increasing slope steepness and the gravity head moves at a velocity that is $60 \%$ as large as that of the following flow. This leads to head growth due to velocity convergence into the head. Below $0.5^{\circ}$ steepness the head and gravity current gradually decreases because frictional forces overcome the gravitational force.

\subsubsection{Numerical modeling}

Although the physical modeling of katabatic flow never became a dominant form of analysis, the same cannot be said for its numerical counterpart. The rapid increase in the use of numerical techniques to represent the atmosphere has essentially kept pace with the growth of computing power since the 1950's. Modeling of katabatic flow, however, started with Thyer in 1966, and grew slowly until the latter part of the 1970's when the most intense U.S. investigation of katabatic flows began with the Atmospheric Studies in Com- 
plex Terrain (ASCOT) program. This, combined with the rapid advances being made with computer technology and numerical methods for fluids, seems to have caused a blossoming of computer application to katabatic flow. Since that time simulations of katabatic flow have increased in resolution, realism and magnitude from sources world wide. Interestingly, although U.S. and British participation in the investigation of katabatic flow until the 1950's could be characterized as marginal (Hawkes, 1947), recent U.S. technological advances in computing have (both in power and application to meteorological instrumentation) allowed its role in the study of katabatic flow to increase dramatically.

\section{Thyer 1966}

From the vantage point of current computing capability the efforts of Thyer (1966) to model katabatic flow numerically seem incredibly bold. As the first attempt it is a further surprise to read that he modelled in both 2- and 3-dimensions. His computer model was hydrostatic and used horizontal grid spacing of $100 \mathrm{~m}$ and vertical grid spacing of $100 \mathrm{~m}$. Unfortunately, he was only able to integrate for two minutes (4 timesteps!) of simulation time due to what was then a very large computing problem. His primary domain represents a 'half-valley' where symmetry is assumed, and has $8 \times 4 \times 10$ grid points $(1000 \mathrm{~m}$ high and $800 \mathrm{~m}$ long by $400 \mathrm{~m}$ wide). Another run was $10 \times 5 \times 12$. In this short execution time he was able to reproduce some basic radiatively driven flow features; up and downslope flow for heating and cooling, respectively, as reproduced in Figure 2.8. Notably the model produces a jet structure although wind speeds were only able to reach $0.20 \mathrm{~m} \mathrm{~s}^{-1}$. The Courant-Friedrichs-Lewy (CFL) criteria for numerical model time integration, for a grid spacing of $100 \mathrm{~m}$ and a timestep of 30 seconds, gives a maximum allowable wind speed of $3.33 \mathrm{~m} \mathrm{~s}^{-1}$. Given this limitation, the size of Thyer's domain and the minimal 


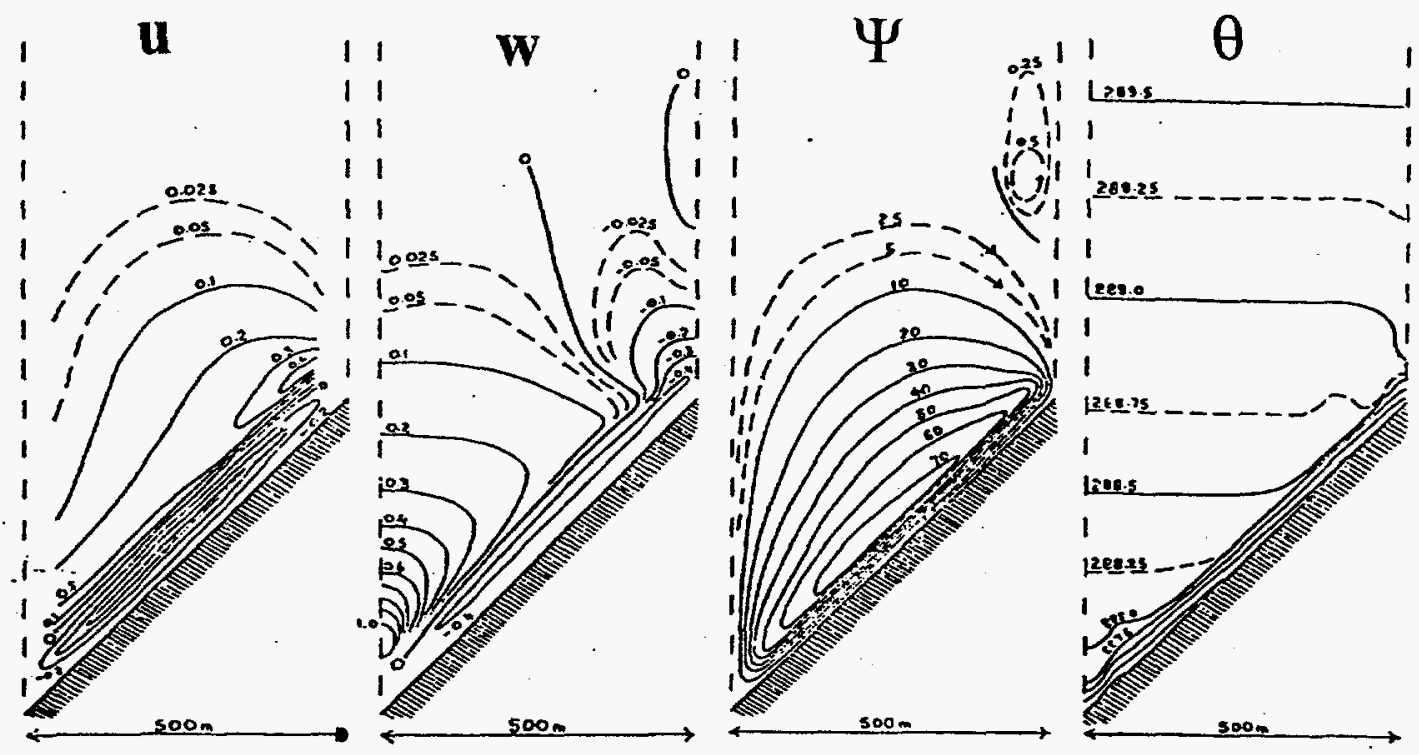

Figure 2.8 Results from the first attempt at numerically modeling katabatic flows from Thyer (1966). Contours of the north-south (v) component of wind speed in $0.05 \mathrm{~m} \mathrm{~s}^{-1}$ intervals are shown. Though small in magnitude the qualitatively expected jet structure of katabatic flow is reproduced.

power of computers at that time, it is something of a feat that he even attempted these sim-

ulations whatsoever.

\section{6-1980}

Compared to the frequency of katabatic flow modeling studies from 1981 on, the rate of publication in the 15 years following Thyer in 1966 was very low. Despite the reasonable success of Thyer's simulations, limited computing resources prevented most subsequent modeling investigations from simulating the atmosphere with as complex a set of equations as Thyer (1966) until the late 1970's. For example, Shieh (1971) reverted to a simple one-dimensional formulation to investigate the transition from down valley katabatic flow to up valley katabatic flow. Assuming a balance between pressure gradient and diffusion with various heat source/sink configurations he found that the down valley wind decays 
from the top downward as it progresses toward a full up valley flow, qualitatively confirming that mechanism as proposed by Davidson and Rao (1963). Another interesting approach to katabatic flow modeling was introduced by Macomber and Ledbetter (1975) who given topographic data and roughness calculate 'velocity factors'. Then, for a given surface cooling, the profile of velocity with height at various locations along the slope can be determined. Although this method showed reasonable success in comparison to observation, the difficulty of its application to real three-dimensional cases limits its applicability.

The transition to somewhat more complicated models of katabatic flow is made by Mahrer and Pielke (1977) who, by transforming their experience with modeling sea breezes (another thermally forced phenomena subject to Bjerknes circulation theorem) to thermal contrasts over sloping terrain, successfully reproduce crude katabatic flows with a two-dimensional hydrostatic model. This model, written in terrain following coordinates, became an integral part of the Regional Atmospheric Modeling System (RAMS) which is used in this study, and was an improvement upon the original formulation coded by Pielke (1974). This model also contained parameterizations for radiation, soil, turbulence and the surface layer. Horizontal grid spacing was 7.5 kilometers for 30 grid points whereas the vertical grid spacing began at a 5 meter depth and expanded vertically to $1000 \mathrm{~m}$ over 12 grid points to $6000 \mathrm{~m}$. We can gain even further appreciation for how ambitious Thyer's (1966) modeling attempts were for the computer power at the time when we consider that some of his simulations contained 600 nodes $(10 \times 12 \times 5)$ a full 10 years before Mahrer and Pielke's study which used 324 nodes $(30 \times 12$, but with far more complicated parame- 


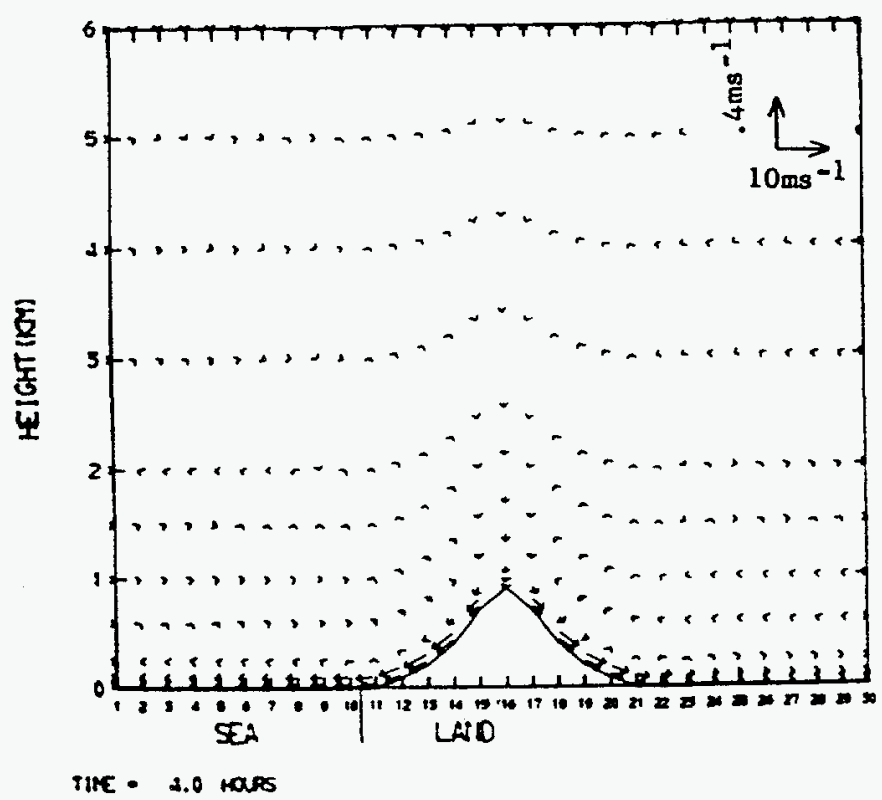

Figure 2.9 A realistic katabatic flow simulation by a hydrostatic model as displayed through wind vectors. Note that air over the peak of the mountain seems to supply the katabatic flow that is evident on the mountainside. From Mahrer and Pielke (1977).

terizations and run for 24 hours).

Mahrer and Pielke's (1977) results for katabatic flow 5 hours after a 1900 LST start are shown in Figure 2.9 (the authors incorrectly use the terminology 'mountain wind' to describe this flow; the phenomena described by the term 'mountain wind' requires a valley configuration which is impossible in two dimensions, though the terminology was more ambiguous at that time). The figure indicates many realistic features of katabatic slope flow predicted by theory and observed in nature, 1) a near surface jet of $4.0 \mathrm{~m} \mathrm{~s}^{-1}$ and 2) a much weaker, deeper return flow aloft. One feature inconsistent with theory, is the generally subsident motion for a great distance above hill top which appears to 'supply' additional mass to the upper slopes as they cool. In contrast, the theory of Wagner (1938) from sequential modifications of theory from Fournet (1840), Wenger (1923) and Wagner (1932a) suggests a horizontal supply source for katabatic flow air from the valley center. Mahrer and Pielke (1977) conclude that sea breeze and thermally forced flows can act in 
concert to produce a more intense circulation as consistent with observations. This verifies the capability of their model for realistic applications.

\section{1-present}

The two-dimensional application of numerical modeling to katabatic flow is also used by Yamada $(1981,1983)$ in his simulations of observations taken over a nearly twodimensional ridge, Rattlesnake Mountain, near Richland, Washington. These observations were taken as part of the ASCOT program and were reported by Horst and Doran (1981) for cases with ambient winds opposing and following the drainage flow. The results compare well with tower observations indicating that with opposing ambient wind the katabatic slope flow is of greater depth and speed than predicted by analytical models (the analytical models do not contain any ambient flow influence). An upstream recirculation zone was also predicted for opposing flow. For following flow the katabatic flow appears to approximate a linear superposition of the ambient flow speed and the katabatic flow analytical solution and has limited turbulence. This author notes that these findings are consistent with the lowering of Richardson number and subsequent increased chance for turbulence when shear is high (opposing flow).

A number of somewhat idealized model results follow the Mahrer and Pielke (1977) and Yamada (1981) contributions. Rao and Snodgrass (1981) present a one-dimensional drainage flow model where a height dependent diffusion coefficient is used in an effort to be more realistic. Although their results match certain observations and the theoretical predictions of Prandtl (1942), they suggest it is applicable mostly to equilibrium states on long, isolated slopes. Also, using a 1-dimensional model Fitzjarrald (1984) finds that an opposing flow retards the development of katabatic flow with some significant simplifica- 
tions. The one-dimensional tactic is also used by Heilman and Dobosy (1985) to simulate a portion of the ASCOT Geysers observations. Using fine resolution, parameterizations of the forested canopy, soil, radiation and turbulence (Mellor-Yamada 1974, classification level 3) they find that ambient conditions are very important to katabatic flow simulations. In particular, they find that horizontal flow structure assumptions strongly effect the results through the vertical advection terms. They show that the effect of flow over surrounding terrain prevents their model from simulating anything but the most quiescent cases accurately.

The first venture into 3-dimensional katabatic flow modeling, since the pioneering study of Thyer (1966) that is, is found in McNider and Pielke (1984) who used an early hydrostatic version of RAMS to simulate valley drainage flows ideally and realistically. Consistent with theory, they find that cooling in the valley center is largely due to upward motion in the valley center and that slope flows decrease as the night wears on. As in observations, valley flows filled the valley to ridge top and developed later than the katabatic slope flows. Despite these successes and qualitatively matching observations in the GoreEagle Valley of Colorado by Whiteman (1981), the predicted wind speed was too low, and the depth was too large. Further, the combination of flows caused shear and thermal advection near the top of the slope flows resulting in continuous turbulence.

Returning to two-dimensions but applying the first non-hydrostatic model to katabatic flow was Bader et al. (1987) who used yet another early version of RAMS to simulate the boundary layer over complex terrain for yet another ASCOT location, the Brush Creek Valley, Colorado studies of 1982 and 1984. Due to the use of a non-hydrostatic version, these investigators were, for the first time, able to simulate the entire diurnal cycle of the 
valley atmosphere. A low-level weak drainage flow develops and grows throughout the night. Shear is maintained between the drainage and the ambient flow. In the morning the $\mathrm{CBL}$ grows under the drainage and eventually destroys it, mixing upper-level winds through the shear zone. This mixing happens first at high elevations where stability is weaker and the stable layer more shallow. These results quantitatively agree with observations in many respects, but are limited by the use of two-dimensions, and perhaps relatively coarse vertical grid spacing, in simulating the higher katabatic down valley flow speeds observed in Brush Creek during those experiments. Bader and McKee (1992) expand on the earlier study by utilizing a wind component into a barrier subject to nocturnal cooling. They find that a two-layer structure develops in which a $75-175 \mathrm{~m}$ deep inversion layer with drainage flow resides under a less stable layer 200-300 $\mathrm{m}$ deep. The shear between the drainage flow and flow aloft appears to create the less stable zone (transition layer) and its depth is proportional to wind speed. The two-layer structure forms regardless of daytime mixed layer depth and the persistent katabatic flow in the lower layer forms due to mechanical and thermodynamic sheltering. Mechanical sheltering is the reduction in wind speed at a particular location caused by surrounding topographic obstacles. Thermodynamic sheltering is the effect of reducing ambient wind effects at a particular location due to strong vertical thermal stability.

Nappo and Rao (1987) use a series of 2-d inclined slope simulations in a very revealing study of katabatic slope flows developing within initially still atmospheric conditions. Their model solves the governing equations for slope parallel flow and the results are framed within the hydraulic approach of Ellison and Turner (1959), Manins and Sawford (1979a) and Briggs (1981) for comparison purposes. Being 2-dimensional the authors are 
able to use the highest resolution thus far in katabatic flow simulation history; covering a domain of $1500 \times 300 \mathrm{~m}$ with $31 \times 31$ grid points $(\Delta x=50 \mathrm{~m}$ and $\Delta z=10 \mathrm{~m})$. Notably, the model is initialized with the Prandtl (1942) steady state profile of wind speed and temperature deficit (see Section 2.4.1), which is then allowed to balance with the governing equations before steadily cooling to a ground surface temperature deficit of $4^{\circ} \mathrm{C}$. The latter configuration is allowed to evolve to a steady state for slope angles from $2.5^{\circ}, 5^{\circ}, 10^{\circ}, 20^{\circ}$, and $40^{\circ}$ at stratifications of $0.1 \mathrm{~K} \mathrm{~km}^{-1}$ (nearly neutral) and $10 \mathrm{~K} \mathrm{~km}^{-1}$ (essentially isothermal) before analysis. Among their conclusions were, 1) increasing ambient stratification decreases the katabatic slope flow speed, 2) for near-neutral stratification the flow speed, depth and entrainment increase with downslope distance, 3 ) for very long or very steep slopes, greater stratification impedes motion so that a balance is reached between turbulent heat transfer and the downslope-warming with respect to the ambient stratification, 4) the buoyancy deficit is created along the slope as the flow loses heat to the radiatively-cooling ground surface causing the net buoyancy deficit to increase with downslope distance and 5) downslope advection and the turbulent entrainment of warmer ambient air aloft act to reduce the layer temperature deficit so that the greater the stratification the quicker the above balance in point (3) can be achieved.

A number of studies have shown the importance of having an accurate topographic representation of a region to simulate thermally forced flows. Doran and Skyllingstad (1992) use a hydrostatic model to simulate local winds in the Cascade Mountains. They find that slight alterations of the synoptic flow structure over local topography can change the local flow prediction significantly. Capturing local terrain features was very important for forecasting surface winds. Even trying to simulate katabatic winds in a V-shaped canyon with 
smoothed topography can cause an underestimation of flow speed (Atkinson 1995). Based on a tracer study near Athens, Greece, Asimakopoulos et al. (1992) conclude that the large variability in katabatic flows due to terrain features makes pollutant transport problems in those regions very difficult. It is necessary to successfully model the static stability and dynamical development due to these features for good dispersion estimates. Along similar lines, Luhar and Rao (1993), in a dispersion modeling study relevant to the ASCOT 1980 program in Anderson Creek Valley, California, found that a 3-dimensional atmospheric model including topography is necessary to adequately capture the meteorological phenomena affecting dispersion in this valley. Bossert and Poulos (1995) have found that topographic variability greatly affects katabatic flow evolution. Even as katabatic flow encountered a relatively small topographic transition from a $2.2^{\circ}$ slope to a $0.4^{\circ}$ slope its character changed significantly. Somewhat more cold and stable layers on the lesser slope were unable to be penetrated by the incoming katabatic flow causing it to elevate (see also Atkinson 1995). Emphasizing the importance of capturing local slope changes are Brost and Wyngaard (1978) who found that even a 1/1000 slope caused large differences in the simulated nocturnal boundary layer.

A large number of sensitivity studies regarding the effects of orography and stability on the slopeside drainage flows within valleys has recently been reported by Atkinson (1995). Using a $21 \times 21 \times 16$ domain, drainage flows into an inverted Witch-of-Agnesi valley of depths $0-2500 \mathrm{~m}$ were analyzed. Grid spacing was large for this kind of study, $\Delta \mathrm{x}$ and $\Delta \mathrm{y}$ were $4000 \mathrm{~m}$ while $\Delta \mathrm{z}$ was stretched with height from $2 \mathrm{~m}$ to $1000 \mathrm{~m}$ and a timestep of $40 \mathrm{~s}$ was used. An interesting limitation that needs to be placed on these results is that the valley thus formed is atypical because it is open ended - at both ends. Since valleys are most 
often closed at one end and open into either a plain or another valley Atkinson's (1995)

results are primarily applicable to valley side katabatic flows as the paper's title states. His main conclusions are:

1. Katabatic slope flow jet altitude, horizontal and vertical velocity increase with increasing inclination from the horizontal.

2. For a given slope angle katabatic flow speed is generally not a function of stability although in some cases higher stability produces slightly lower flow speeds.

3. The depth of the cool katabatic layer increases with increasing slope.

4. For a constant slope the katabatic layer depth decreases with increasing stability.

5. As distance downslope increases the cooling at the surface increases although the inversion strength decreases due to turbulent mixing.

Result \#2 is in conflict with the results of 1) McNider (1982) who finds that increasing stability will reduce katabatic flow speeds in a modification of the Fleagle (1950) analytical model and 2) Nappo and Rao (1987) who model reduced katabatic flow speed as initial thermal stability rises. Atkinson (1995) also finds that as the valley atmosphere cools the horizontal pressure gradient from slope to mid-valley decreases causing a reduction in slope flow velocity, an effect that was suggested by Wagner (1938).

\section{Physiographic effects}

Since the observational analysis in Section 2.3.4 indicated that thermally forced flows were sensitive to the radiative effects of passing clouds, one might also expect that any factor influencing slope cooling rate, such as clouds, vegetation and soil type, soil moisture, albedo or snow cover, might also influence katabatic flow. Investigations into all of these have been completed in some form, except the influence of soil type. Also, snow cover effects have mostly been studied in reference to glacier winds and dispersion.

The first numerical modeling study of vegetative canopy effects, as opposed to the bulk approach taken by Briggs $(1979,1981)$, was that by Watanabe (1994) who discusses the influence of a sparsely vegetated slope versus a fully vegetated slope on drainage flow 
using a $1-D$ model and a multi-source vegetation parameterization. While admittedly a simple representation of katabatic flow he finds that a sparsely vegetated slope will reduce the katabatic flow relative to either a unvegetated or fully vegetated slope. Therefore, using a single-source (instead of double-source or multi-source) vegetation model will overestimate katabatic flow speeds in a sparsely vegetated region. The wind and temperature profiles in fully covered vegetation for all vegetation parameterization types are very similar but with sparse cover the katabatic flow speeds are $25 \%$ less than full vegetation simulations and cooling is about $20 \%$ less. This reduction is ascribed to the cold air being trapped in the sub-canopy layer due to vegetation-reduced turbulence (and subsequent very stable layers), which prevents turbulent exchange of cold surface air into what would otherwise become a katabatic flow layer. Although the sparse vegetation has a larger $z_{0}$ than full vegetation, the author does not differentiate the effect of the increased $z_{o}$ on flow speed from that of the cold air trapping mechanism. These conclusions essentially agree with Briggs (1981) results once it is noted that Briggs (1981) assumes that the katabatic flow is within the canopy whereas Watanabe (1994) parameterizes the effect of the canopy on katabatic flow above the canopy so far as the numerical model is concerned. Briggs (1981) argues that a katabatic flow layer can exist within the canopy layer such as observed by Cornfeld (1938).

The effect of soil moisture on katabatic flows had not been directly investigated until recent work by Banta and Gannon (1995), although the strength of the effect (as opposed to what the effect would be) could be surmised from Ookouchi et al. (1984), Pielke and Uliasz (1992) and Ek and Cuenca (1994). Banta and Gannon (1995) found that increased soil moisture had a much different effect on local flows during the night than compared to 
during the day. During the day increased soil moisture has the effect of reducing anabatic flows because a larger fraction of the energy input to the atmosphere is used for the latent heat of evaporation. At night however, the higher evaporative cooling as soil moisture increased was offset by two effects, 1) the greater heat conductivity of more moist soil allowed heat within the ground to more easily transmit to the ground surface from below offsetting the cooling and 2) the greater moisture in the atmosphere enhanced the longwave radiation returned to the ground. The result from these effects, primarily the former, was to reduce the cooling rate of the near surface atmosphere and therefore the primary forcing mechanism of katabatic flow. The reduction in katabatic flow speed varied with soil type from $18 \%$ (sandy soil) to $28 \%$ (silty clay loam) and typically affected approximately the first 5 hours of katabatic flow development.

Clouds will alter the radiative characteristics of the atmosphere over a surface so one would expect katabatic flows to be substantially affected by them. The effect of clouds on local flows has been treated generally by Segal et al. (1986) and specific to katabatic flow by Ye et al. (1989). Using both analytical analysis and numerical modeling, Ye et al. (1989) were able to make some strong conclusions for slopes less than $1^{\circ}$ and length less than $100 \mathrm{~km}$ as summarized succinctly in this excerpt from their paper (where $\mathrm{C}$ is the fraction of sky covered by cloud):

"(ii) The depth and the intensity of thermally-induced downslope flows decrease non-linearly as the fraction of cloud cover increases for a given cloud height. Likewise, the flow increases non-linearly with increasing cloud base height for a given cloud fraction coverage. The impact of high clouds on the intensity and depth of downslope flow can be nearly neglected, even under overcast conditions. However, the impact of low clouds on the depth and the intensity of downslope flow under overcast conditions is pronounced.

(iii) A change from clear to cloudy conditions before midnight will alter the development process of the nighttime thermally-induced downslope flow. The downslope flow will cease to develop or even will decay (slowly or rapidly, 
depending on the fraction of cloud cover for a given cloud height). For the low cloud conditions which were simulated, following the appearance of the clouds, with $\mathrm{C}=0.5$ and $\mathrm{C}=1.0$, reduction of the downslope flow by about a factor of $\sim$ 0.2 and $\sim 0.5$ were found, respectively.

(iv) A change from cloudy to clear skies before midnight causes an intensification of the downslope flow. For low clouds, for example, when the clearing of the sky is from $\mathrm{C}=0.5$ and 1.0, respectively, the downslope peak flow values several hours after the clearing were increased by factor of $\sim 2$ and $\sim 5$, respectively, as compared to the corresponding time when no clearing took place."

Clearly, clouds can have a significant effect on katabatic flows although only limited observations of this effect (i.e. Barr and Orgill 1989 observed katabatic canyon flows decreasing to $25 \%$ of their typical depth during low cloud ceiling conditions), usually by happenstance, have been documented (see Ye et al. 1989 and references therein). The case studies selected for investigation in this dissertation do not have any cloud cover or limited high altitude cloudiness, and are therefore do not have significant cloud induced variability.

\subsection{Mountain Waves}

A brief summary of the characteristics of gravity waves caused by the movement of air over an obstacle, often called mountain waves, follows. Since katabatic flows are a surface based phenomena this work is primarily interested in those aspects of mountain waves that have the potential to influence the surface properties in which katabatic flows develop.

\subsubsection{Basic aspects}

Mountain waves are a subset of a much larger regime of atmospheric waves called gravity waves, so called because the fundamental restoring force for them is the gravitational or buoyancy force. As with many concepts that appear quite complex in their various extensions, the roots of gravity wave theory rely on the teachings of traditional physics. Much like the vertical displacement of a mass attached to a vertically oriented spring in 


\section{Gregory S. Poulos}

the classic harmonic oscillator problem, if a mass of air is displaced either downward or upward in a stable atmosphere (to be defined), it will oscillate. The initial displacement will determine the amplitude of the oscillation and the strength of stability (analogous to the stiffness of the spring or the size of the spring constant) will uniquely determine the frequency of oscillation, $N$. If one then imagines that same oscillating mass of air moving at a constant horizontal phase velocity, $c_{p}$, throughout the process, the path of the mass of air will form a wave-like shape of wavelength, $\lambda=c_{p} N^{-1}$. These simple ideas lay a foundation for the understanding of gravity waves in the atmosphere. One unique quality about gravity waves is that they incite atmospheric fluctuations in vertical and horizontal velocity and pressure that are $90^{\circ}$ out of phase with oscillations of temperature or buoyancy. One consequence of this quality is that the energy flux of a gravity wave is perpendicular to its direction of motion. Thus, a horizontally propagating gravity wave will transport energy vertically. A large body of literature in sections of various books (Holton 1979, Gill 1982, Dutton 1987 among others) and in peer-reviewed literature (Finnigan and Einaudi 1987, Durran 1990) have discussed the basic aspects of gravity waves.

The fundamental frequency of oscillation, $\mathrm{N}$, in the atmosphere is called the Brunt-Vaisala frequency and is defined by the vertical potential temperature structure as,

$$
N=\left(\frac{g}{\theta_{o}} \frac{\partial \theta}{\partial z}\right)^{\frac{1}{2}}
$$

where $\theta_{0}$ is the base state potential temperature and is on the order of $300 \mathrm{~K}$. For a U.S. Standard Atmosphere potential temperature lapse rate of $3.4 \mathrm{~K} \mathrm{~km}^{-1}$ and gravitational acceleration of $9.8 \mathrm{~m} \mathrm{~s}^{-2}, \mathrm{~N}=0.001 \mathrm{~s}^{-1}$. Oscillations of any frequency less than $\mathrm{N}$ are sta- 
ble; those of frequency greater than $\mathrm{N}$ are unstable and will decay.

Quite often a dimensionless quantity called the Froude number, Fr, is defined for mountain wave flows. The Froude number uses Brunt-Vaisala frequency in its definition,

$$
F r=\frac{U}{N H}
$$

where $\mathrm{U}$ is the mean wind speed impacting a barrier of height $\mathrm{H}$. This formulation can therefore be thought of as the ratio of kinetic energy to potential energy of the atmosphere. It has been found that linear theory of mountain waves can be applied successfully where Fr $<0.1$ and $\mathrm{Fr}>0.9$ (Queney et al., 1960). In this dissertation various Froude numbers in the range $0.0-1.2$ will be used in attempts to classify MKI behavior relative to Froude number. Unfortunately it has been found that the majority of the days over the Rocky Mountains can be classified in the non-linear regime where $F$ falls between 0.1 and $\sim 0.8$ (see Section 3.4).

The linear theory of mountain waves has been severely limited in comparisons with real atmospheric data, particularly with the most noteworthy mountain wave effect, severe downslope windstorms, by the use of Fr-type dependence in its formulations. This is because there seldom exists a day where atmospheric stability and windspeed are reasonably constant from the near surface up to mountain top. With this in mind, Scorer (1949) extended the linear theory, using the Boussinesq approximation, to include wind shear and $\mathrm{N}$ variations with height in what is now called the Scorer parameter, $l$,

$$
l=\left(\frac{N^{2}}{u_{o}}-\frac{1}{u_{o d z}} \frac{d^{2} u_{o}}{{ }^{2}}\right)^{\frac{1}{2}}
$$

where $u_{o}$ is the horizontal wind which is a function of $z$. The introduction of this more 
realistically varying atmospheric structure allowed Scorer (1949) to predict the presence of trapped lee waves when the Scorer parameter of a lower atmospheric layer of depth $\mathrm{H}$ is sufficiently larger than the $l$ of the layer above. Note that for an unsheared atmosphere, as either stability goes up or wind speed goes down, the Scorer parameter increases while the Froude number decreases.

\subsubsection{Atmospheric perturbations due to mountain waves}

The Scorer parameter has been used to relate mountain wave behavior to the underlying topography (Smith 1979) in the linear theory of flow over two-dimensional sinusoidal ridges. For the case where the topographic wavelength, $\lambda=2 \pi / \mathrm{k}$, is smaller than the Scorer parameter $\left(\right.$ or $\mathrm{k}^{2}>l^{2}$ ) the disturbance caused by the topography will have a frequency greater than can be supported by the intrinsic stability, N. In this case, which is also analogous to a narrow valley, the wave is evanescent with height and its phase lines have little tilt from the vertical. This is a non-hydrostatic regime and its requirements are more easily met for a given valley as $l$ decreases $\left(l\right.$ decreases for smaller $\mathrm{N}$, larger wind speed $\mathrm{u}_{0}$, and greater vertical shear). As the topographic wavelength increases (or the valley widens) for the same atmospheric conditions, and $\mathrm{k}$ decreases toward $l$, the mountain waves are less and less evanescent with height. When $\mathrm{k}^{2}<l^{2}$ (the topographic wavelength is greater than l) the perturbation by the topography is of a frequency less than, and therefore supported by, the Brunt-Vaisala frequency. This allows topographically-induced gravity waves to propagate vertically without decay, transporting energy upwards. Wave motions will tend toward hydrostatic and there is a drag on the atmosphere induced by low wind/high pressure on the upwind side of the barrier versus high wind/low pressure in the lee. Mountain wave drag has become known to be an important forcing on the general circulation and is 
often parameterized in global circulation models.

The pressure perturbation associated with mountain waves has been addressed with both observations and analytical solutions. Queney et al. (1960) discuss linear solutions and shows the surface perturbation of atmospheric fields associated with various mountain sizes and waves at constant $\mathrm{u}=10 \mathrm{~m} \mathrm{~s}^{-1}, \mathrm{~N}=0.01 \mathrm{~s}^{-1}$ and $\mathrm{H}=1 \mathrm{~km}(\mathrm{Fr}=1.0)$. Figure 2.10 (also shown in Chapter 1) shows two such solutions. The mountain wave solution for a barrier of $10 \mathrm{~km}$ half-width $\left(\mathrm{k}^{2}<l^{2}\right)$ shown in Figure 2.10a has a strong vertical signature that neither damps with height nor propagates downstream. In contrast, for the same initial atmospheric conditions but a mountain of $1 \mathrm{~km}$ half- width $(\mathrm{k} \sim l)$ the mountain wave perturbation propagates both vertically and downstream (although the reader should note the horizontal scale change between the figures). In each case the ground level pressure and
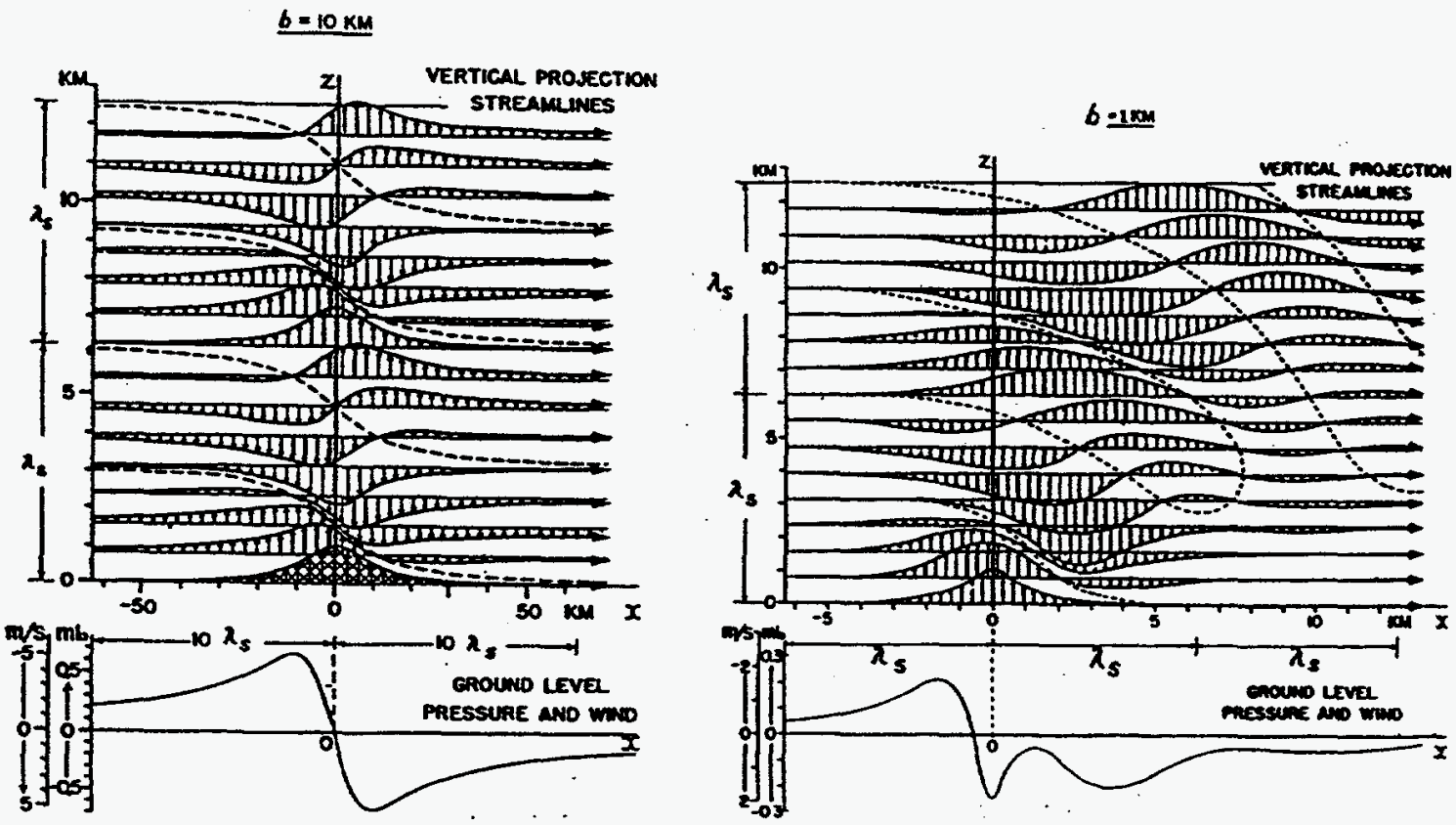

Figure 2.10 Froude number $=1.0$ flow over a $1 \mathrm{~km}$ high obstacle whose width is (a) much larger relative to the lee wavelength and (b) close to the lee wavelength. Note that various patterns of surface perturbation arise for different mountain size. (Queney et al. 1960). 
velocity perturbations behave oppositely as expected for gravity waves (see also Durran 1990). The magnitudes of the velocity and pressure perturbations are significant; approximately $5 \mathrm{~m} \mathrm{~s}^{-1}$ and $0.5 \mathrm{mb}$ respectively, for these conditions. Since typical values of these atmospheric variables in katabatic slope and valley flows are about $5 \mathrm{~m} \mathrm{~s}^{-1}$ for wind speed and up to $2.0 \mathrm{mb}$ for pressure perturbation relative to the free atmosphere, one can see that mountain wave flow superimposed on katabatic flow will have a significant effect. The perturbation high pressure on the upwind side is caused by adiabatic cooling of upward flowing air above the surface. Furthermore, since buoyancy perturbations are out of phase with the velocity and pressure perturbations one would expect potential modulation of the buoyancy field of a developed katabatic flow by overlying mountain waves. Although the description of Queney (1960) emphasizes barrier width relative to mountain wave wavelength, Baines (1987) points out that for cases relevant to the atmosphere - continuously stratified flow and infinite depth - the important parameter is Froude number and the length and shape of the obstacle are of lesser importance (Pierrehumbert 1984, Pierrehumbert and Wyman 1985, Peltier and Clark 1984).

The atmospheric response to a barrier in relatively high Fr flow as just described differs considerably from that response at low Fr. If the barrier is essentially a 2-dimensional ridge and $\mathrm{Fr}$ is below approximately 0.8 some fraction of the lowest levels of flow is unable to surmount the barrier (discussed as 'blocking' below). The more three-dimensional a barrier is, the more opportunity there is for flow that cannot rise over the barrier to flow around the barrier. For lower $\mathrm{Fr}$, a greater portion of the flow must flow around rather than over the barrier. The pressure perturbation patterns around a 3-d obstacle differ in many respects from the pressure perturbations for higher Fr flow and can vary greatly with 
topographical shape and the actual Fr (Drazin 1961). In general for 3-d obstacles, there is an upwind perturbation high pressure, lower pressure at the sides and a somewhat higher pressure downwind (Smith 1979). Low Fr flow is essentially non-linear and has been found to cause vortex shedding downwind which has been investigated by Chopra (1973), Smolarkiewicz and Rotunno $(1989,1990)$ and Rotunno and Smolarkiewicz (1992). The large 2-d ridge represented by the Rocky Mountains west of the ASCOT 1993 study area is expected to produce mostly blocking type atmospheric responses with fewer 3-dimensional effects.

Blocking, a low Fr flow regime where some portion of the flow does not surmount the obstacle, has a different effect on katabatic flow than those cases where the flow is not blocked. In the blocking case, where the barrier is approximately 2-dimensional, flow cannot easily go around the barrier and a mesohigh pressure forms on the upwind side. Blocking has been generally found for $F<0.70$, although according to Sheppard (1956) any flow with $F<1.0$ is partially blocked (Baines 1987). However, Smith (1990) shows that Sheppard's argument is erroneous. His reasoning primarily relies on the fact that Sheppard's (1956) concept requires the pressure of the rising air parcel to be equal to that of free air at an unperturbed location away from the mountain throughout ascent and descent over the barrier. However, from the previous discussion and figures, it is clear that mountain waves do, indeed, induce pressure perturbations at the surface. In further support of Smith's (1990) argument, it is convincing to note that Sheppard's (1956) formula predicts the lowest wind speeds at mountain top and slower speeds in general so long as the parcel is at a higher altitude (i.e. more potential, less kinetic energy) than its original level - an effect that has never been observed. The recirculatory/return flow zone that develops in a 
region of blocked flow on the upstream side of a barrier, will not oppose katabatic flow direction suggesting that a different turbulent interaction is likely compared to unblocked flow. Blocking for the Rocky Mountains has been found to occur $50-85 \%$ of the time in winter during westerly flow by Mayr (1993) and it is therefore significant to the problem at hand.

\subsection{KaTABatiC FLOW-MOUNTAIN WAVE INTERACTION}

Of primary interest in this dissertation is the interaction of mountain waves with katabatic flow. While little previous work of this kind has directly been attempted before, there have been numerous studies of the effects of ambient or overlying winds on katabatic flow where reference to mountain waves has occurred. In some sense, since most katabatic flow investigations occur in topographically complex regions, most overlying flow is inherently affected by mountain wave dynamics. The majority of studies below fall into the category of studying the effects of so-called ambient flow with katabatic flow.

\subsubsection{Strong ambient winds}

As described by Wagner (1938), the Ph. D. dissertation of Bondy (1935) investigated conditions of strong gradient winds and katabatic flow for the Inn Valley. Bondy (1935) found that during foehn conditions, katabatic flow was non-existent but that katabatic forcing could be found in a nighttime wind direction shift to more down-valley. During the day a wind shift in the opposite sense occurred. Other studies verify this effect (Kanitscheider 1936) but even suggest that a katabatic westerly flow will develop in the Inn Valley underneath a westerly foehn. In agreement with this concept, Banta and Cotton (1981) have shown that drainage winds can develop underneath overlying westerlies (at least in

westerlies less than $10 \mathrm{~m} \mathrm{~s}^{-1}$ ) as temperatures drop and an inversion develops in South 
Park, Colorado. Wagner (1938) suggests that it is conceivable that the pressure distribution forced by the foehn enhances the down valley pressure gradient. Here we have one of the first indications that a mountain wave phenomena could be reinforcing a katabatic flow. Regardless of the wind phenomena in the Inn Valley, a valley-plain pressure difference that affects the flow can be detected. In deep valleys, Wagner (1938) finds that, with the exception of strong winds, the gradient wind will not generally influence the katabatic down valley wind. He argues that the pressure gradients causing the katabatic flow are so large over such relatively short distances that they overwhelm the synoptic pressure gradient. A climatological study of downslope katabatic flow in Denmark (Mahrt and Larsen 1990) confirms that katabatic flows can develop underneath ambient winds from any direction, even for a slope as small as $1^{\circ}$. Regardless of whether the ambient flow is strong enough to prevent the downslope flow from occurring, the effect of katabatic forcing on the flow is still evident.

\subsubsection{Ambient wind opposing katabatic flow direction}

Balloon soundings indicate that when the gradient wind opposes the down valley katabatic wind a wind minima near zero occurs at the height where the katabatic pressure forcing decreases to that of the synoptic pressure gradient (Ekhart 1944). As described by Hawkes (1947), an investigation over small hills in England by Cornfeld (1938, see Figure 2.11a and b) showed that an opposing wind in the lee could create a calm zone along the hillside, where, without ambient flow, a katabatic flow would otherwise reside. Katabatic flow was found to exist below this calm zone but ambient flow dominated to the top of the hill. Although the discussion by Wagner (1983) did not discuss the weakening or strengthening of katabatic flow by ambient winds, Ellison and Turner's (1959) physical modeling 
study showed that opposing overlying flow reduced downslope wind speed. If strong enough, opposing flow prevented downslope flow from occurring. Observations of katabatic flow in opposing flow by Neff and King (1989) showed a reduction in valley drainage wind depth, an effect that is corroborated by Mursch-Radlgruber (1995, see Figure $2.11 \mathrm{c})$

A layer average modeling study of both shooting and tranquil katabatic slope winds in opposing flow was completed by Fitzjarrald (1984). In agreement with Ellison and Turner (1959) he finds that katabatic slope flows can be either slowed appreciably or negated by opposing flow. Depending on the cooling rate of the slope the start up time for tranquil flows can be significantly delayed (until buoyancy forces are able to overcome ambient forcing. Furthermore, in cases where katabatic flow developed underneath opposing flow, they were found to steadily entrain and deepen although their flow speed did not increase in time. From this study we find that ambient flow opposing katabatic slope flow can severely impact katabatic flow development although, as found by Wagner (1938), eventually katabatic forcing due to surface cooling dominates.

Fitzjarrald's (1984) results were expanded upon by Arritt and Pielke (1986) who used a one-dimensional model with vertical structure and a turbulence parameterization to investigate the interaction of ambient winds with katabatic flow. They conclude that the turbulent interaction at the katabatic flow boundary is a key element in determining the reaction of katabatic flow. For a given opposing ambient flow the katabatic thermal forcing is found to be stronger due to reduced shear below the jet although, in agreement with Fitzjarrald (1984), the actual katabatic flow speed was weaker. An approximation of this effect by linear superposition of ambient wind speed onto the quiescent katabatic flow speed was 

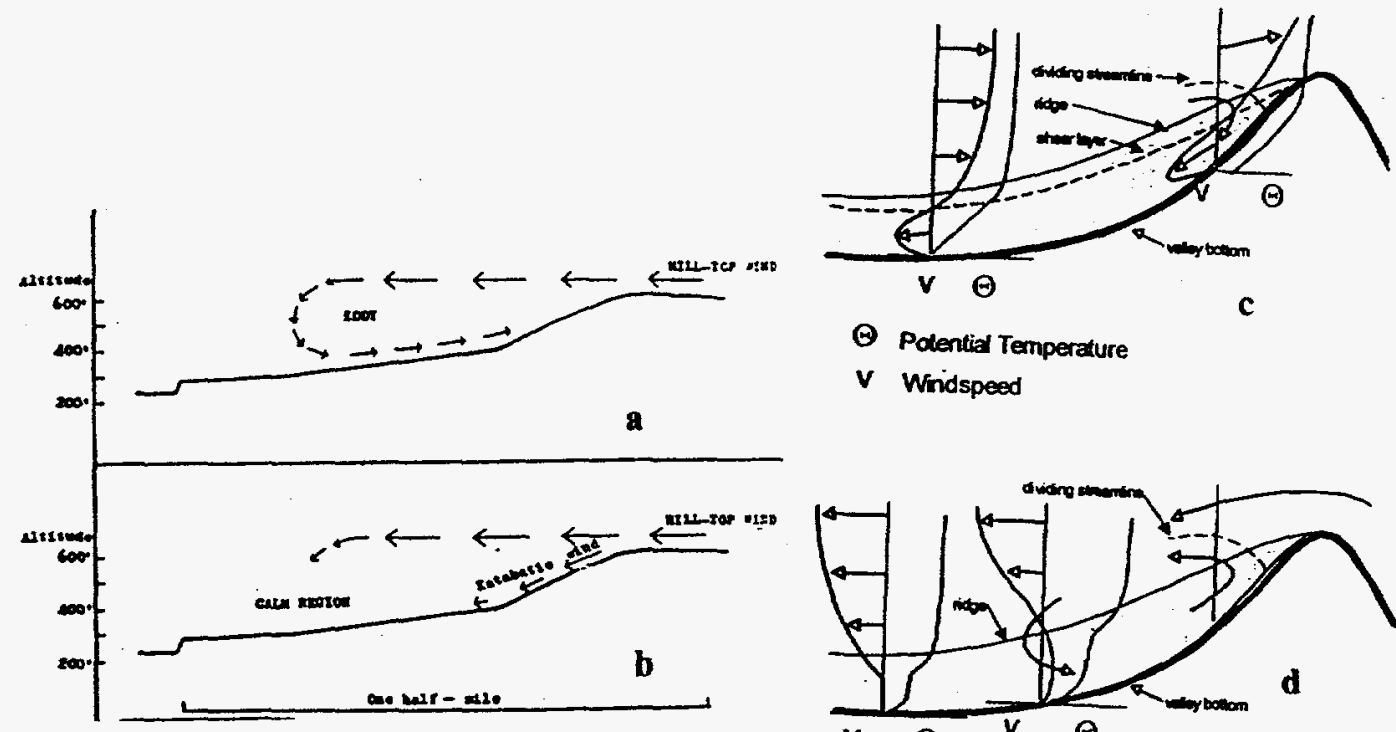

$\Theta$ Potential Temperature

$\checkmark$ Wridspeed

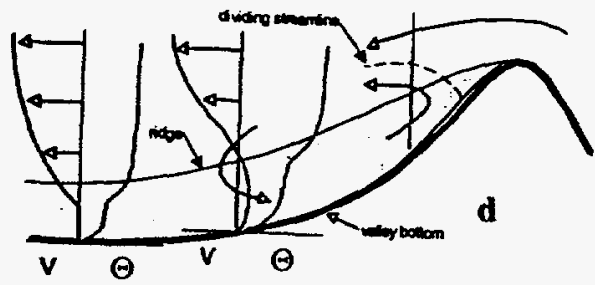

Figure 2.11 A comparison of figures a) and b) from Cornfeld (1938) for two ways aiding flow can impact katabatic slope flow and for c) opposing and d) aiding ambient winds overlying katabatic flow from Mursch-Radlgruber (1995). In c) and d) the solid line labeled 'ridge' is the valley side wall height or ridgeline, and it delineates the depth of local flows in the undisturbed case.

found to be inaccurate indicating a non-linear response. Retaining a prognostic equation

for turbulent kinetic energy allowed the model to contain turbulent transfer across the

katabatic flow jet) whereas typical Richardson number dependent eddy viscosity formula-

tions would not. Arritt and Pielke's (1986) modeling results have been verified observa-

tionally in a recent tethersonde analysis of katabatic flow by Mursch-Radlgruber (1995).

In opposing flow, though katabatic flow is shallower, its speed is not significantly reduced

compared to the undisturbed case, indicating that katabatic forcing has actually increased

relative to quiescent conditions (see Figure 2.11 ).

The effect of opposing flow on vertical thermal structure has also been investigated by

Bader and McKee (1992) who study the development of the nocturnal boundary layer in 
2-dimensions over a slope under conditions of opposing ambient flow. They find that a two-layer structure develops: a 75-175m deep inversion layer containing drainage flow of a few meters per second develops under a transition layer of less stable layer 200-300 m deep. The shear between the drainage flow and flow aloft appears to create the less stable transition layer and its depth increases proportionally with increasing opposing wind speed. This two-layer nighttime structure develops regardless of daytime mixed layer depth and is due to mechanical sheltering and the strong temperature inversion.

Orgill et al. (1992) use observations in Brush Creek Valley, Colorado from the 1984 ASCOT experiment to conclude that the main factors contributing to the erosion of valley drainage depth were the magnitude of the opposing wind, valley stability and the acceleration of above valley opposing flow. An intensifying opposing ambient flow of at least 1.44 $\mathrm{m} \mathrm{s}^{-1}$ hour ${ }^{-1}$ was found to be very conducive to turbulent erosion of drainage to at least half its undisturbed depth. Otherwise ambient opposing flow of $5 \mathrm{~m} \mathrm{~s}^{-1}$ were required to significantly decrease drainage depth. A $5 \mathrm{~m} \mathrm{~s}^{-1}$ wind threshold was also found by Gudiksen et al. (1992) to strongly interfere with the development of katabatic flows at Grand Mesa, Colorado. They found that at this threshold turbulent mixing of near surface temperatures did not allow enough cooling for the development of katabatic flows.

\subsubsection{Ambient wind aiding or following katabatic flow direction}

For a gradient wind in the same direction as the katabatic wind Wagner (1938) finds that a minima still occurs in the vertical profile of horizontal wind, but it is located where the sum of the ambient and katabatic pressure gradients minimizes. Hawkes (1947) claims that the strong stability of katabatic flow prevents aiding ambient flow from having a very deep effect. Cornfeld's (1938) observations of katabatic flow in aided flow indicated that a 
recirculatory eddy on the lee side of a hill (this was discussed earlier as opposing flow as well) could manifest itself either immediately near the hill top preventing katabatic flow there or further down the hill side creating a calm at that point (where katabatic slope flow exists between hilltop and the calm). Such an effect has been observed in a much more sophisticated observational fashion by Mursch-Radlgruber (1995, see Figure $2.11 \mathrm{c}$ and d). Mursch-Radlgruber's observations suggest that the dynamic pressure gradient caused by flow over topography in a high mountain Austrian valley opposes the katabatic flow producing a calm or even flow reversal, though pressure measurements were not made. Differently, for an aiding ambient wind without any topographic wave effects Arritt and Pielke (1986) found that the total near-surface wind speed was higher (representing the effect of katabatic forcing plus aiding flow) giving stronger mixing across the katabatic jet and reducing the katabatic forcing.

In valleys the effect of aiding winds on katabatic flows can be variable. For Coal Creek Canyon Colorado (see Figure 2.12), Coulter and Gudiksen (1995) report that the katabatic

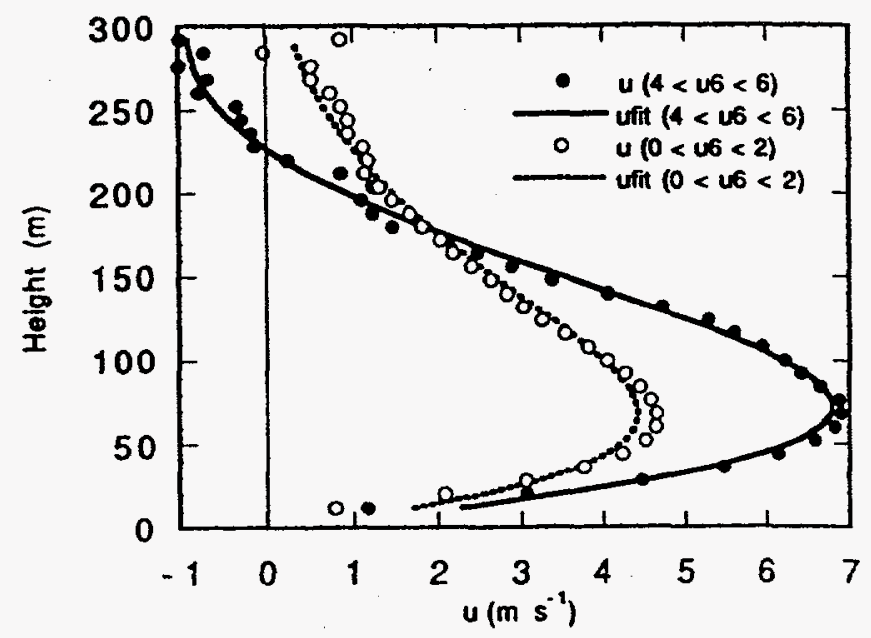

Figure 2.12.Drainage flow speed versus height for Coal Creek Canyon, Colorado based on 3 year averages of sodar data. The dark curve and filled dots represent the profile with an aiding wind between 4.0 and $6.0 \mathrm{~m} \mathrm{~s}^{-1}$ and the dashed curve with open dots shows the profile for external wind (u6) less than $2 \mathrm{~m} \mathrm{~s}^{-1}$ (Coulter and Gudiksen 1995). $u 6$ is the wind speed measured by a high elevation (mountain top) tower used to indicate external forcing strength 
flow depth $(250 \mathrm{~m})$ and jet height $(70 \mathrm{~m})$ maximize with an external aiding wind of $3 \mathrm{~m} \mathrm{~s}^{-}$ ${ }^{1}$, but the katabatic flow depth decreases below this external wind strength. The katabatic flow speed maximum increased as external aiding flow increased above $3 \mathrm{~m} \mathrm{~s}^{-1}$. Three years of observed sodar data from the mouth of Coal Creek Canyon was analyzed to produce these conclusions. Neff and King $(1987,1989)$ show that ambient winds can have varying effects when intruding into a tributary versus the main canyon depending on orientation. In this case, as aiding upper level winds increased to $7 \mathrm{~m} \mathrm{~s}^{-1}$, there was a halving of the katabatic flow speed in the main valley, similar to the Mursch-Radlgruber (1995) observations. In a side valley perpendicular to the overlying flow, drainage was nearly eliminated.

\subsubsection{Ambient wind perpendicular to katabatic flow direction}

Wagner (1938) further argues that a cross valley ambient wind will be detected suddenly as one measures vertically through a down valley katabatic fiow. As a consequence of adiabatic warming as a mountain wave descends into the stably stratified valley a sharp inversion aloft will be created through which the gradient wind cannot penetrate thereby creating a distinct shear layer between itself and the valley flow. Obviously the strength of dynamic forcing influences penetration depth and Hawkes (1947) suggests this penetration is generally minimal. In contrast, the physical and numerical modeling study by Bell and Thompson (1980) found that a stably stratified valley may be swept out ('ventilated') if the Froude number exceeds 1.3 for valley perpendicular flow. Ventilation or flushing is found to occur when the inertial forces dominate the buoyancy forces independent of valley side slope. Observational evidence acquired by Neff and King (1989) agrees with the Bell and Thompson (1980) result. For Anderson Valley, California cross valley flows were 

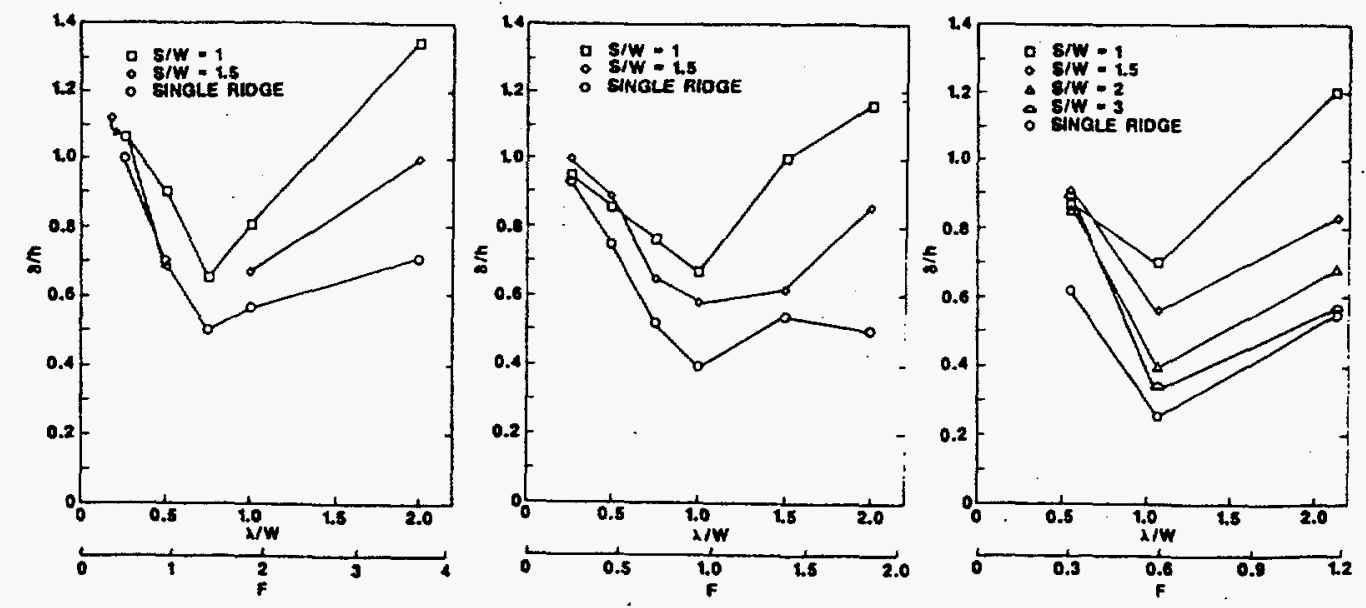

Figure 2.13 Variation in depth of penetration normalized by valley depth for different Froude numbers (Lee et al. 1987). a) for gentle ridges, b) for moderately steep ridges and c) for steep ridges (that approximate the steepness of Brush Creek Valley, Colorado).

found to significantly reduce drainage flow depth as they penetrated into the valley,

although no Froude number analysis was completed.

Lee et al. (1987) use the EPA Fluid Modeling Facility to investigate the flow over 3-

dimensional elongated ridges in stable stratification. They found a distinct separation zone that varied in behavior for differing slope angles or pairs of ridges forming a valley. For varying Froude number flow the cross valley flow was able to penetrate different fractions of valley depth as shown in Figure 2.13. S/W is the ratio of ridge separation to ridge width (larger values of $\mathrm{S} / \mathrm{W}$ indicate a greater distance between ridges) and $\lambda / \mathrm{W}$ is a measure of stability similar to Froude number. These figures show that a lone 2-dimensional ridge.(open circles) allows the lowest penetration of ambient flow into a lee-side stable zone regardless of ridge steepness. Furthermore, the closer two ridges become the less the penetration becomes because the mechanical forcing of the other ridge is felt over a shorter distance. This in effect shows that narrower valleys mechanically protect the flows 
within them more strongly than wider valleys. Interestingly, for $\mathrm{S} / \mathrm{W}=1.0$ the deepest penetration of ambient flow was the same, about 0.7 , regardless of ridge steepness.

\subsubsection{For off valley axis ambient flow directions}

Doran (1991) uses the Clark (1977) non-hydrostatic mesoscale model to investigate valley katabatic flows with overlying winds from varying directions. His approach is to model the ASCOT Brush Creek 1984 experiment with an idealized valley shape. Winds were initialized as a logarithmic profile to valley depth $(700 \mathrm{~m})$, and were allowed to spinup to the topography for 2 hours, before commencing with surface heat flux induced cooling based on the Brush Creek experiments. For simulations where ambient flow was systematically increased from $0.5 \mathrm{~m} \mathrm{~s}^{-1}$ to $6.0 \mathrm{~m} \mathrm{~s}^{-1}$ oriented $60^{\circ}$ left of directly up-valley, katabatic flow depth and strength reduces significantly with increased wind speed as shown in Figure 2.14a. Doran (1991) explains that this behavior is consistent with turbulent mixing and subsequent weakening of both the inversion and the katabatic forcing. Similar behavior was found for directly opposing wind direction although this was only tested for 2 and $4 \mathrm{~m} \mathrm{~s}^{-1}$ windspeeds. When ambient flow speed is kept constant but orientation relative to the up valley direction is changed from $0^{\circ}$ to $60^{\circ}$, as in Figure $2.14 \mathrm{~b}$, the sensitivity of katabatic flow depth is minimal while katabatic flow speed is not well behaved. Directly up-valley winds led to deeper stronger katabatic flow which Doran (1991) ascribes to lesser turbulent interaction (flow 'rides over' the katabatic flow) allowing greater cooling and therefore stronger winds, although a mountain wave dynamic effect similar to that of Mursch-Radlgruber (1995) could well be operating in this case. This result is partly at odds with the results of Arritt and Pielke (1986) and Fitzjarrald (1984) who find that for opposing flow katabatic flow depth and forcing increase while the 

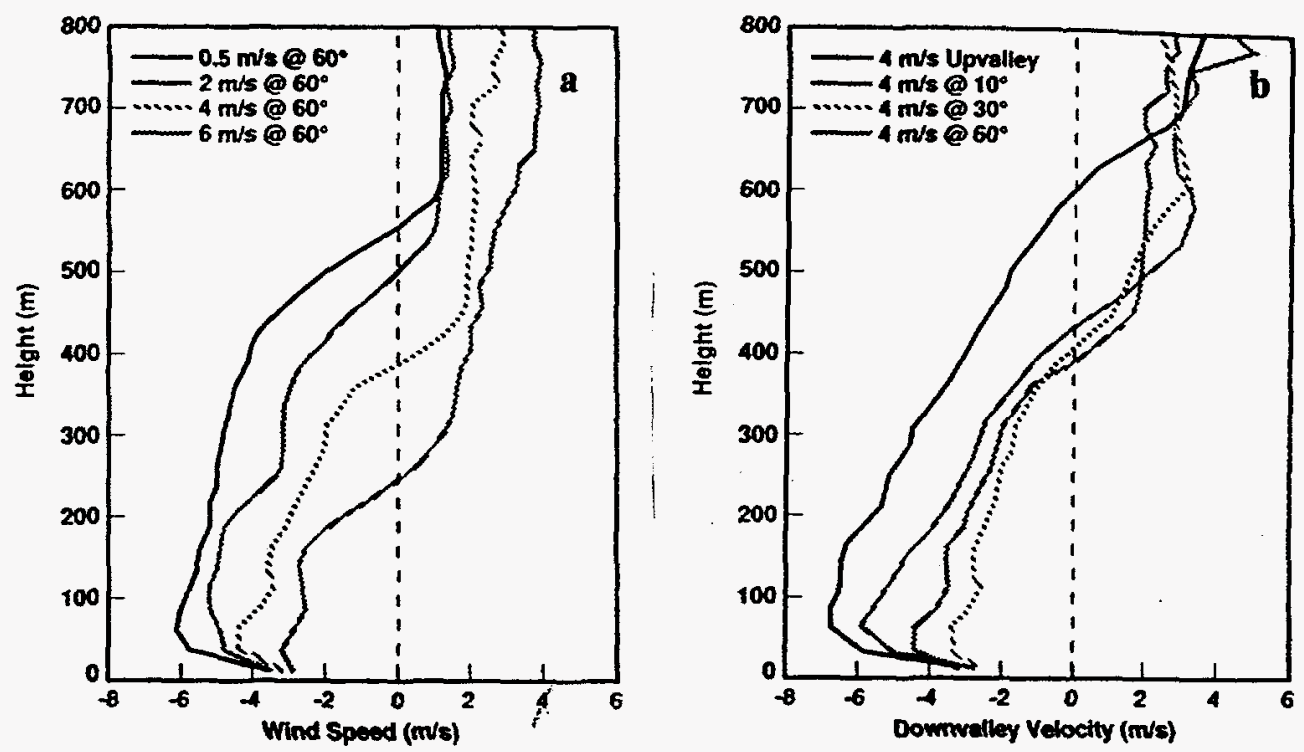

Figure 2.14 The change in katabatic valley flow structure for a) fixed orientation, $60^{\circ}$ left of directly up-valley at various speeds and, b) at $4 \mathrm{~m} \mathrm{~s}^{-1}$ for various orientations relative to the up valley axis. These modeling results from Doran (1991) clearly show ambient wind effects.

actual katabatic flow speed reduces. Referring back to Figure $2.14 \mathrm{~b}$, when the ambient

wind is at greater angles to the valley greater mixing occurs as flow descends into the valley (mountain wave effect) and erodes it somewhat (an increase in turbulent exchange).

That valley katabatic wind depth varies with flow aloft has been observationally shown by Davidson and Rao (1963). They find that about $50 \%$ of the statistical variability in the depth of the katabatic valley flow can be explained by variations in the $1000 \mathrm{~m}$ wind for an $800 \mathrm{~m}$ deep valley near Manchester Center, Vermont.

Orgill and Schreck (1985) describe the Geysers area experiments and the interactions of overlying winds with katabatic flow. In particular, they find that occasional synoptic easterlies would interact with the low levels and disrupt the drainage in Anderson Creek.

Wind flow, stability and temperature were all affected. They found that mountain waves caused by flow over local topography were fully capable of eroding or flushing out katabatic flows over a period of one to two hours. At times overlying flow was found to 
create shear conditions sufficient to cause turbulent erosion from above. This erosion process was less severe than the mountain wave flushing mechanism, and could take place over several hours.

The katabatic winds in the more topographically sheltered Brush Creek Valley, Colorado were also found to be influenced strongly by overlying flow. An in-depth study by Barr and Orgill (1989) describes many of the potential interactions as ambient flow overrides valley drainage flow. With light ambient winds the drainage depth fills the valley to ridgeline depth $\mathrm{H}_{\mathrm{o}}$. However, as the strength of the ridgetop wind, $\mathrm{v}$, increases, the depth, $\mathrm{H}$, of valley drainage is found to decrease according to the empirical formula,

$$
H=H_{o}(1-0.068 \mathrm{~V}) \text {. }
$$

showing that external winds are of considerably greater influence on Brush Creek's

katabatic flow than those of the Vermont valleys studied by Jaffe (1958) and Davidson and Rao (1963). Barr and Orgill (1989) use measurements of the height of the valley drainage to graphically correlate this height to ambient Froude number (shown in Figure 2.15). We see in the figure that as Froude number increases toward 1.0 the drainage depth steadily decreases regardless of wind direction. Although this represents a limited data set, it is a unique and thorough use of experimental resources that shows a variety of mountain wave types critically impacting drainage. Barr and Orgill (1989) go on to show that their analysis of the importance of mountain wave penetration on valley katabatic flow also applies to the Anderson-Putah Creek Valley observed during the 1980 ASCOT experiments. They further find that cross valley ambient winds can induce helical circulations that severely complicate wind fields compared to standard models of undisturbed down canyon flow. These circulations prevent simple dispersion calculations from being successful even in 


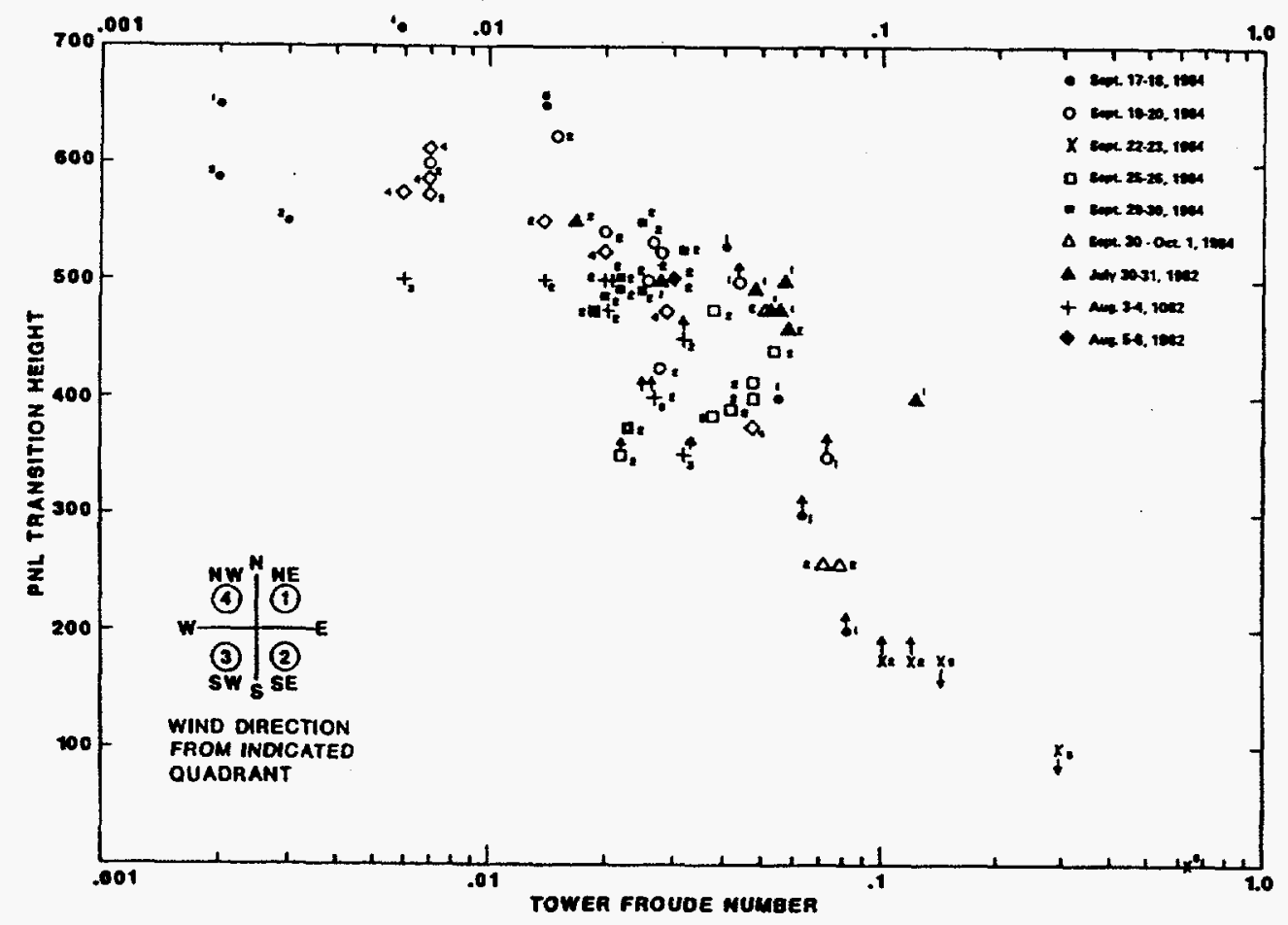

Figure 2.15 Variation in depth of drainage flow in Brush Creek Valley for different Froude numbers as calculated from observations (Barr and Orgill 1989).

deep confined canyons such as Brush Creek, as also found by Start et al. (1975).

\subsubsection{Variability induced by overlying flow}

Davidson (1963b) observed rapid variability in katabatic flow along a lee slope during varying ambient flow conditions, presumably with some mountain wave effects. Considerably greater katabatic flow variability has also been found in numerical simulations of slope flow (Bossert and Poulos 1995) when an overlying ambient wind is included. Davidson (1963b) also observed that as the flow stabilizes overnight the downwind separation zone moves uphill and turbulence reduces significantly - a result consistent with stabilization sequentially impeding the flow of air over the hill. Some results using a two-dimen- 
sional mesoscale model (Ying and Baopu 1993) have shown that the nocturnal phase of cooling may indeed alter the overlying mountain wave. Ying and Baopu (1993) find that at night a mountain wave which during the day was aided by the development of a convective boundary layer (CBL) in rising over the mountain may not be able to surmount the obstacle due to developing stability. There results appear to show that there is a non-linear coupling between mountain wave and katabatic flow phenomena, although this is not described in detail.

\subsection{SUMMARY}

In this chapter a review of previous work was presented. From the description of katabatic flow research it is clear that these flows are pervasive worldwide and have a long history of investigation. Dynamically, katabatic flows can be explained by the Circulation

Theorem; a result that supports the original explanation by Fournet (1840). Because a horizontal pressure gradient directed away from the slope or out of a valley, caused by the cooling of near surface air, is the forcing mechanism, katabatic flows are subject to alteration by external influence on the local pressure gradient force. External influences such as mesoscale pressure gradients, ambient winds, clouds, roughness, vegetation and mountain waves have been considered, to varying degrees of scrutiny. This review also gave background and a history of mountain waves, though to a lesser depth than the review of katabatic flow. Finally, a review of studies that give insight on mountain wave - katabatic flow interaction $(\mathrm{MKI})$ was presented, revealing that it is not clear how these two complex terrain phenomena evolve. The main goal of this work is to extend the limited knowledge of how mountain waves and katabatic flow interact using observations and modeling. 


\section{CHAPTER 3}

\section{ObSERVATIONAL ANALYSIS}

This chapter reviews the observations taken near the Colorado Front Range in the period July 1993 - April 1994. A number of experiments and existing networks were operational during this period in addition to the primary experiment, the ASCOT 1993 Rocky Flats campaign. Information from some of these data sources can be used for phenomenological analysis or model result evaluation. After an introduction to the data sources, the case days of interest for this study are reviewed and summarized. An analysis of upstream conditions for the Colorado Rockies is presented in climatological form to evaluate potential mountain wave conditions for these case studies.

\subsection{Primary Data Source: ASCOT (7/93-3/94)}

The majority of data used in this study comes from the 1993 ASCOT field program held near Rocky Flats and Boulder, Colorado. The observational network is shown in Figure 3.1 and, although slated to continuously operate only from August-November, 1993, was left in place until the spring of 1994 (with a smaller portion of the instruments operating during the extended period). There were 3 primary observing platforms: sodars, wind profilers and towers. These instruments were placed strategically in and around the two Front Range canyons of interest to ASCOT investigators, Coal Creek Canyon and Eldorado Canyon. The wind profilers were oriented in a roughly east-west line from the 


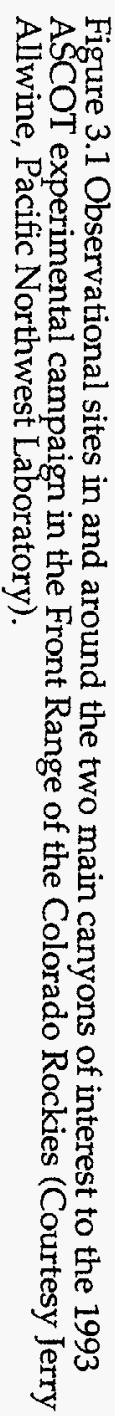

\section{Front Range Instrument Locations}

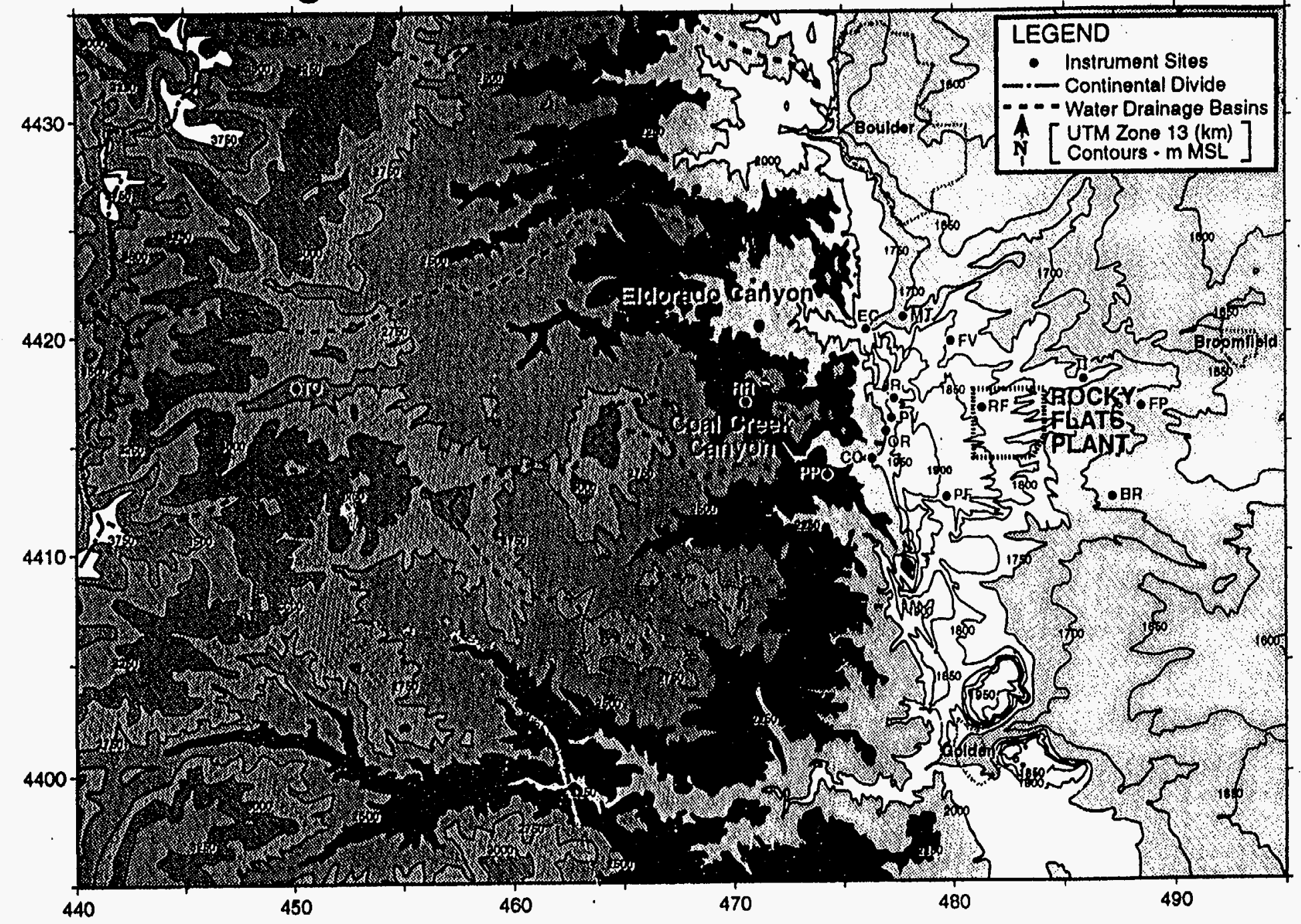


Continental Divide east (originally the profiler line was intended to cross the Divide, but operational problems prevented this) to capture larger-scale flow features.

\subsubsection{Tower data}

Data from the 10 towers (see Table 3.1 ) were collected in hourly averages over the observational period. The locations of the towers was such that flow in less accessible canyon regions (near canyon heads, along slopes, etc.) could be determined. Towers were mostly instrumented at two levels (each $<25 \mathrm{~m} \mathrm{AGL}$, but typically $6 \mathrm{~m}$ and $17 \mathrm{~m}$ ) allowing the determination of low level inversion strength between the two heights. In addition to wind speed, direction, temperature and relative humidity, most towers also recorded net radiation - a key factor in the development of katabatic flows.

\subsubsection{Sodar data}

The sodars were the primary observational tool for the katabatic flows that developed in the Coal Creek and Eldorado Canyons. For this reason a sodar was placed at the mouth of Coal Creek Canyon as indicated in Figure 3.1. Additionally, three sodars were placed radially about $2 \mathrm{~km}$ from the mouth of Eldorado Canyon in approximate $30^{\circ}$ increments in order to capture the non-stationary movement of the drainage flow as it exited the canyon. By recording wind direction, wind speed and vertical motion with height and time, drainage evolution (over 15 minute averages) was captured. This allowed a continuous monitoring of katabatic flow structure, intrusions from overlying flows and the important twice-daily transitions between up and downslope flow.

\subsubsection{Wind Profilers/RASS}

The line of profilers generally produced wind direction and speed data for $500 \mathrm{~m}-4000$ m AGL, with occasions where a greater range was possible. The RASS associated with 
some of the profilers covered a somewhat smaller range from $500 \mathrm{~m}$ to $2000 \mathrm{~m}$ AGL. This range inconsistency unfortunately did not allow for local Richardson number calculations which would have been very useful for assessing the potential for turbulent interactions.

The profiler array does allow for the plotting of useful wind time series with height, although vertical cross-sections were not possible due to inconsistent performance. More complete model comparisons to observations can be made using this data.

\section{2 additional Data Sources}

In addition to the ASCOT 1993 data source, a number of additional coincident data sets were taken by other organizations. A number of these are used in supplementary fashion.

\subsubsection{NCAR Mesonet}

The long running NCAR mesonetwork of surface based towers was operational during the ASCOT field program. This network provides approximately 30 surface observing stations on the plains, along the foothills and occasionally in the mountains. The data recorded is standard (wind speed, direction, temperature, relative humidity and pressure).

\subsubsection{NWS Soundings and Surface Observations}

The National Weather Service operates a number of useful instruments in the Colorado region. Of most use is the twice daily rawinsonde launches ( $00 \mathrm{Z}$ and $12 \mathrm{Z})$ from Denver and Grand Junction. These soundings are used in this dissertation to provide an important basis for evaluating mountain wave conditions in the region of interest. A climatology of Froude numbers based on this consistent data set for 28 years is discussed in Section 3.4 below and further detailed in Appendix B.

\subsubsection{NOAA Aeronomy Laboratory Photochemistry Experiment (8-9/93)}

NOAA's Aeronomy Laboratory (NOAA/AL) was conducting some high altitude instru- 
ment verification experiments in August and September of 1993 (Olson et al. 1996, Baumann et al. 1996), overlapping the main observational period of ASCOT 1993.

Measurements of standard atmospheric variables as well as chemical constituents $\mathrm{O}_{3}, \mathrm{NO}$ and $\mathrm{NO}_{2}$ were recorded for three stations at approximately $3000 \mathrm{~m} \mathrm{MSL}$ just east of the Continental Divide near Boulder, Colorado. The chemistry information could be used to verify source regions for air at high elevations for a number of katabatic flow nights. The wind data is useful to determine when high altitude scouring of cold air source regions by westerly component flow is occurring and potential effects on katabatic flow strength.

\subsubsection{Colorado AIRS Sites (6/92-3/94)}

Combined with the NOAA/AL data set, the chemistry information contained in the AIRS data set can be used to evaluate stagnation days and detect the diurnal variation of pollutants along the Front Range. The numerous meteorological towers, although not as routinely quality controlled, are useful to determine the existence of katabatic flows on the plains and to provide an even denser observational network than exists with NWS, NCAR Mesonet and ASCOT measurement sites alone.

\subsubsection{NOAA/Forecast Systems Laboratory (7-10/93)}

NOAA's Forecast Systems Laboratory (NOAA/FSL), through Dr. Jerome Schmidt, has provided 6 hourly gridded data sets based on the numerous Front Range observations. These 3-d volumes of atmospheric data are suitable for using in the RAMS model in fourdimensional data assimilation mode. They are generated by a constantly running system at FSL called the Mesoscale Analysis and Prediction System (MAPS) which interpolates the observations onto the 3-d grid (Bleck and Benjamin 1993, Pan et al. 1994). The observations may come from any number of sources including the NCAR Mesonet, radar, wind 
profilers, aircraft observations or standard NWS soundings. The MAPS data is used for four-dimensional data assimilation (4DDA) in this study when a simulation of an actual case day is made, in order to obtain the most accurate initial and external boundary condition (which will then inherently contain changing large-scale conditions) during the simulation. The horizontal grid increment of the MAPS data is $60 \mathrm{~km}$ horizontally and varies vertically, covering the entire U.S.

\subsubsection{Winter Icing and Storms Project (1-3/94)}

The Winter Icing and Storms Project (WISP), which has been investigating the potential for aircraft icing due to the microphysics of Front Range cold season storms since 1990, was in operation coincidentally with the latter portion of the ASCOT observations in early 1994. The WISP data set includes a number of radars, additional towers, the Wyoming Highway network and aircraft. Some days during WISP investigated mountain wave conditions along the Front Range with aircraft and allow additional insight into the potential for mountain wave/katabatic flow interaction on a limited set of nights. This is an excellent, quality-assured data set that supplements the ASCOT data during the 1994 period. although it is not used here because we focus on the earlier ASCOT period.

\subsection{Case Study Days}

For the purposes of this dissertation, overnight periods where katabatic flow was evident for some portion of the night are of interest. As noted in Chapter 2, there exist nights where synoptic or mountain wave forcing flushes katabatic winds completely from a slope or canyon, but since katabatic forces are not dominant in this case, such instances are not investigated here. In particular, cases of relatively undisturbed katabatic flow and those where the likelihood of mountain wave interaction with katabatic flow was high, but not 
dominant, are sought. An objective and subjective hunt was made through the many days of the observational campaign (ASCOT only) to identify days on which katabatic flow existed. Katabatic flow was evident on over $40 \%$ of all case nights. As regards mountain wave information, time-varying wind and temperature information via a wind profiler located at Gross Reservoir (approximately $10 \mathrm{~km}$ west of the mouth of Eldorado Canyon in Figure 1) or Tolland (the 'TO' in Figure 1) were scheduled to be used. Unfortunately the wind profiler data is wrought with bad information and is unusable. For this reason upstream soundings from Grand Junction were used to calculate the Froude number of flow over the Continental Divide.Three different case nights from the many available nights of the August to November period were selected. They were chosen to represent the condition where, 1) there was little or no interaction with weak ambient flow aloft as a baseline case, 2) where ambient, upstream flow was significant and causing a mountain wave, but katabatic flow dominated low levels and 3) there were periodic breaks in the drainage condition due to mountain wave erosion but drainage generally dominated. By investigating these cases some documented ambient flow influence on drainage flow was found.

\subsubsection{Drainage Flow Characterization}

The data primarily used in choosing katabatic flow case nights is mini-sodar data from the mouth of Coal Creek Canyon (the ' $\mathrm{CC}$ ' in Figure 3.1), the tower instrumented at two levels at Rim Rock (6.10 m and $17.68 \mathrm{~m}$, the 'RR' in Figure 3.1) and, to provide upper level information, synoptic charts from 850 to $200 \mathrm{mb}$ and Grand Junction soundings. An objective and subjective selection methodology was employed because objective predictors of good drainage flow nights have been found to be imperfect (Gudiksen 1989). The 
subjective analysis uses time-height cross sections of wind speed, wind direction, vertical motion at the Coal Creek mini-sodar and the author's impressions of the likelihood of a particular night being drainage flow. The objective ranking scheme evaluates higher elevation meteorological characteristics from the Rim Rock tower site to determine how conducive they are to the production of good katabatic canyon flows. The characteristics of the tower data calculated for the objective drainage flow evaluation in Coal Creek Canyon were; 1) 6pm to 6am LST average net radiation, 2) 6pm to 6am LST average lapse rate between the $6 \mathrm{~m}$ and $17 \mathrm{~m}$ tower levels, 3) temperature range, 4) 6pm to $6 \mathrm{am}$ LST standard deviation of the wind direction, $\sigma_{\theta}$ and 5) the $6 \mathrm{pm}$ to 6am LST average wind speed. The above values were then sorted for all nights from August through November 1993 to find a range of values. Then the values were sorted from most conducive to least conducive to drainage flow. Once sorted a particular case night was ranked relative to the scale of values and given a average ranking on a scale from 1.0 to 10.0 , where 1.0 indicates the best drainage conditions. Oppositely, the subjective ranking scheme, described below, used a ranking from 10 to 1 , where a ' 10 ' represents the best drainage night. While this difference can be confusing, please be aware of it in the following discussion.

For the subjective selections a minisodar height-time plot such as depicted in Figure 1.2 on Page 4 was judged for its quality of features which indicate drainage flow. In this figure midnight is at the ' $24^{\prime}$ ' on the $\mathrm{x}$-axis and the $270.0^{\circ}$ contour in Panel $b$ is roughly indicative of down Coal Creek Canyon direction (actually $278^{\circ}$ ). This particular night, 02-03 Oct 1993, was judged highly (a ' 9 ') by the author. The following features were considered indicative of a good drainage night, 1) the existence of a consistent surface-based flow with a jet from transition to transition (top panel of Figure 1.2), 2) the existence of an 
afternoon transition from upslope flow and a morning transition to upslope flow (middle panel of Figure 1.2),3) the direction of winds during the overnight period were consistently from the expected drainage direction based on canyon orientation (middle panel of Figure 1.2), 4) the existence of significant downward vertical motion overnight (lower panel of Figure 1.2) and 5) that the features in numbers 1, 3 and 4 above occurred simultaneously. These criteria are all met, to varying degrees, for the three cases described below.

Figure 1.2 shows that the wind direction changes quickly during the afternoon transition to the full depth of the drainage almost simultaneously as has been observed (Wagner 1938, Buettner and Thyer 1965), but the drainage accelerates for some three hours before reaching approximately steady state (with the exception of the acceleration later in the night). By 1830 LST, the depth of the drainage flow, the westerly winds and downward vertical motion became essentially the same; and their depths continue to be similar throughout the night indicating how strongly the thermally driven flow dominates the canyon atmosphere. Toward morning the drainage flow speed weakens with height just before a complete transition to upslope flow at 0715 . At transition there is a lull in the wind speed down to a minimum of less than $1 \mathrm{~ms}^{-1}$, and upward vertical motion associated with upslope flow quickly dominates the daytime canyon atmosphere. As shown below this case night was chosen as representative of an excellent, undisturbed drainage night.

Table 3.1 is a list of the score, rank and meteorological attributes of the top 30 objective selections from all available tower data. For drainage to be objectively likely (a low score) a particular night would have high negative net radiation, high average lapse rate, high daily temperature range, low $\sigma_{\theta}$ and low wind speed from the Rim Rock tower data. One might alter the variables chosen as more is learned about what determines the best 
Table 3.1: The top 30 likely katabatic flow nights based on an objective ranking scheme of data from the Rim Rock meteorological tower. Boldface indicates those nights described in this chapter. The average lapse rate was calculated as the temperature difference between the $17 \mathrm{~m}$ and $6 \mathrm{~m}$ heights. Columns 7 and 8 used the $6 \mathrm{~m} \mathrm{AGL}$ observational level. The Froude number was calculated from the Grand Junction, CO daily balloon sounding as described in Section 3.4.

\begin{tabular}{|c|c|c|c|c|c|c|c|c|}
\hline & & & Average & Average & Daily & & & $\nabla$ \\
\hline & & & Net & Lapse & Temp & $\operatorname{Avg}(6 \mathrm{~m})$ & Avg & Froude \\
\hline \multirow[t]{2}{*}{ Rank } & Objective & Date & Radiation & Rate & Range & $\sigma_{\mathrm{q}}$ & Wind Spd & Number \\
\hline & Score & mmdd & $\mathrm{W} \mathrm{m} \mathrm{m}^{-2}$ & $\left({ }^{\circ} \mathrm{C} \mathrm{km}^{-1}\right)$ & $\left({ }^{\circ} \mathrm{C}\right)$ & $\left({ }^{\circ}\right)$ & $\mathrm{ms}^{-1}$ & \\
\hline 1 & 1.73 & 1004 & -62.9 & 88.5 & 19.1 & 15.1 & 1.0 & 0.28 \\
\hline 2 & 1.82 & 0927 & -75.5 & 128.1 & 18.2 & 34.8 & 2.0 & 0.00 \\
\hline 3 & $\overline{1.94}$ & 0903 & -66.9 & 96.4 & 19.3 & 29.4 & 1.0 & 0.00 \\
\hline 4 & 2.02 & 1005 & -58.0 & 79.9 & 18.6 & 15.1 & 1.0 & 0.63 \\
\hline 5 & 2.05 & 0911 & -71.2 & 77.7 & 20.4 & 29.9 & 1.1 & 1.20 \\
\hline 6 & 2.13 & 0929 & -70.4 & 115.9 & 17.7 & 37.8 & 1.5 & 0.34 \\
\hline 7 & 2.29 & 0823 & -72.5 & 100.0 & 17.5 & 43.5 & 0.8 & 0.72 \\
\hline 8 & 2.36 & 0822 & -66.3 & 77.0 & 16.1 & 27.3 & 0.8 & 0.68 \\
\hline 9 & 2.38 & 1003 & -64.4 & 116.6 & 16.8 & 43.7 & 1.0 & 0.08 \\
\hline 10 & 2.45 & 0920 & -64.2 & 96.4 & 17.6 & 38.9 & 1.0 & 0.32 \\
\hline 11 & 2.48 & 1023 & -62.8 & 68.4 & 17.1 & 24.8 & 1.0 & 0.00 \\
\hline 12 & 2.51 & 0824 & -56.2 & 87.1 & 16.4 & 27.7 & 0.9 & 0.25 \\
\hline 13 & 2.64 & 1107 & -67.8 & 75.6 & 16.5 & 34.7 & 1.1 & 0.45 \\
\hline 14 & 2.74 & 1002 & -54.4 & 36.7 & 21.1 & 22.5 & 0.9 & 0.00 \\
\hline 15 & 2.76 & 1116 & -34.1 & 79.9 & 21.2 & 23.9 & 1.0 & 0.09 \\
\hline 16 & 2.77 & 0915 & -65.1 & 93.6 & 16.1 & 48.9 & 0.5 & 0.00 \\
\hline 17 & 2.84 & 0928 & -60.3 & 26.6 & 16.8 & 13.8 & 1.1 & 0.13 \\
\hline 18 & 2.86 & 0806 & -56.2 & 63.3 & 17.6 & 26.7 & 1.4 & 0.00 \\
\hline 19 & 2.87 & 0828 & -55.0 & 78.4 & 13.9 & 28.1 & 0.8 & 0.00 \\
\hline 20 & 3.03 & 1022 & -55.3 & 44.6 & 15.6 & 20.6 & 1.1 & 0.00 \\
\hline 21 & 3.03 & 1006 & -44.8 & 49.7 & 16.9 & 16.7 & 1.1 & 0.00 \\
\hline 22 & 3.08 & 1110 & -53.5 & 52.5 & 17.6 & 28.0 & 1.2 & 0.21 \\
\hline 23 & 3.14 & 1109 & -69.2 & 84.9 & 16.8 & 55.1 & 1.3 & 0.63 \\
\hline 24 & 3.15 & 1121 & -49.1 & 67.6 & 17.2 & 34.3 & 1.0 & 0.22 \\
\hline 25 & 3.24 & 0919 & -64.4 & 119.5 & 13.7 & 67.2 & 0.6 & 0.42 \\
\hline 26 & 3.27 & 0914 & -68.9 & 78.4 & 17.2 & 60.9 & 0.9 & 0.00 \\
\hline 27 & 3.36 & 0930 & -68.2 & 29.5 & 21.5 & 42.0 & 2.0 & 0.86 \\
\hline 28 & 3.44 & 0807 & -52.2 & 43.9 & 15.0 & 29.1 & 1.1 & 0.38 \\
\hline 29 & 3.49 & 1021 & -52.9 & 111.5 & 15.9 & 66.1 & $\overline{0.7}$ & 0.00 \\
\hline 30 & 3.50 & 0801 & -51.5 & 18.0 & 13.9 & 16.1 & 1.1 & 0.33 \\
\hline
\end{tabular}


drainage. As mentioned above, unlike the objective rankings by computer, the author's subjective process ranked katabatic flow nights by whole numbers, from 10 to 1 , where a ranking of ' 10 ' was applied to an excellent drainage night. A comparison (not shown) of the top 30 subjectively ranked katabatic flow nights with Table 3.1 shows considerable agreement with a number of exceptions. 20 of the top 30 nights subjectively chosen are in the top 30 nights selected objectively. Four of the ten differences can be accounted for by data incompatibility (i.e. data for analysis does not exist at Rim Rock but does for Coal Creek or vice-versa) between the data sets.

There are then six case nights in each table that are not in the other and that have no immediate explanation. While some are not particularly good drainage nights in any case, some nights that were subjectively excellent drainage nights do not rank well objectively. Perhaps the most intriguing differences lies in the two subjectively excellent drainage nights of 02 Sep and 07 Sep 1993 which do not rank in the top thirty of the objective analysis. This difference may imply an overemphasis on the importance of lapse rate versus the importance of low wind speed aloft (see Table 3.1) but will not be investigated further here.

\subsubsection{Case 1 - Undisturbed katabatic flow: October 02-03, 1993}

This case night represents what might be called a 'classic' drainage night and was described somewhat in the previous section. Weather conditions during 02-03 October 1993 were quiescent over the study area. Surface synoptic maps (not shown) indicated that near surface winds were light and variable up to $5 \mathrm{~m} \mathrm{~s}^{-1}$. The area wide sea level adjusted pressure averaged near $1023 \mathrm{mb}$ reflecting the high pressure that had moved in after an earlier weak frontal passage. The slight cloud coverage that existed in late after- 
noon dissipated overnight as surface temperatures on the plains dropped from around $18^{\circ} \mathrm{C}$ to near $6^{\circ} \mathrm{C}$ overnight. Around the ASCOT study area on 03 October conditions were clear with variable winds up to $3 \mathrm{~m} \mathrm{~s}^{-1}$ and temperatures nearing $26^{\circ} \mathrm{C}$. Conditions are further described in Appendix A. These quiescent conditions were ripe for drainage flows and the description of sodar observations in the previous section bears this out.

Local conditions were also conducive to drainage flow, so it is natural that this night ranked a ' 9 ' in my subjective category and is 9 th in the objective rankings ( see boldface in Table 3.1). Radiatively, this night was characterized by 12 hours of $55.3 \mathrm{~W} \mathrm{~m}^{-2}$ average net negative radiation indicative of strong cooling which generates a high average lapse rate of $44.6^{\circ} \mathrm{C} \mathrm{km}^{-1}$ in the lowest $20 \mathrm{~m} \mathrm{AGL}$. (Note that this indicates the rate of increase in temperature with height between the two levels on the Rim Rock tower, $T_{17.68 m^{-}}$ $\left.\mathrm{T}_{6.10 \mathrm{~m}}\right)$. Tower winds at $6 \mathrm{~m}$ AGL for the 12 hour period had low variability $\left(\sigma_{\theta}=20.6^{\circ}\right)$ and light average speed of $1 \mathrm{~m} \mathrm{~s}^{-1}$ indicating quiescent well directed (i.e. by local terrain) winds. Synoptic charts from 850 to $200 \mathrm{mb}$ indicated much lighter than average wind speeds, from 3 to $17 \mathrm{~m} \mathrm{~s}^{-1}$, respectively. These light winds are verified by the low Froude number calculated for this night from the Grand Junction sounding, 0.08 , in the last column of Table 3.1 (Froude number calculations are described in detail in Section 3.4). The diurnal range of $15.6^{\circ} \mathrm{C}$ indicates mostly clear skies dominated the Coal Creek airshed.

\subsubsection{Case 2 - Katabatic flow with 320 ambient flow: 03-04 September 1993}

This case had a strong mountain wave embedded in northwesterly flow, as well as very consistent katabatic flows within the ASCOT observational network. Their was just one observed intrusion of external air into the katabatic flow overnight, which differentiates this case from the undisturbed case described in the previous section. On the other hand, 
there were far fewer intrusions than in the disturbed case described in the next section.

This case was chosen as the case study for modeling purposes as will be described further in Chapters 6 \& 7 .

The large scale flow at $500 \mathrm{mb}$ and $700 \mathrm{mb}$ from the National Weather Service facsimile products are shown in Figures 3.2 and 3.3, respectively, for 00Z and 12Z 4 Sep 1993. From these figures it is clear that the synoptic situation relevant to the ASCOT study area - a deep low pressure system to the northeast near the Arctic Circle and a high pressure system to the southwest - caused geostrophic northwesterly flow at $500 \mathrm{mb}$ and $700 \mathrm{mb}$. This pattern was very consistent from $00 \mathrm{Z}$ to $12 \mathrm{Z}$, with some slight cold advection $(700$ $\mathrm{mb}$ Denver temperatures dropped from $13^{\circ} \mathrm{C}$ to $10^{\circ} \mathrm{C}$ and those at $500 \mathrm{mb}$ dropped from $8^{\circ} \mathrm{C}$ to $-10^{\circ} \mathrm{C}$ ). $850 \mathrm{mb}$ and surface level plots (not shown) indicated a slight lee trough over eastern Colorado as might be expected for this northwesterly flow. An analysis of the Grand Junction sounding at $00 \mathrm{Z}$ and $12 \mathrm{Z}$ found average flow from $320^{\circ}$ at $\sim 7.5 \mathrm{~m} \mathrm{~s}^{-1}$ in the layer from $2000-4000 \mathrm{~m}$ MSL. Static stability in this layer was near $1.3 \mathrm{~K} \mathrm{~km}^{-1}$ overnight such that a mountain wave of $\mathrm{Fr}=\sim 0.45$ existed, on average during the period. Since the vertical thermal and wind structure was not constant through this layer, and the Grand Junction sounding does not perfectly represent upstream conditions for the Rocky Mountains, one cannot expect exact $\mathrm{Fr}=0.45$ behaviour, but rather generally non-linear conditions with wave breaking likely at times.

During the day prior to this night time case, skies were clear and temperatures reached approximately $30^{\circ} \mathrm{C}$ with very dry (dewpoints $\sim 0^{\circ} \mathrm{C}$ ) conditions. Generally light, southeast winds were indicative of gentle upslope winds. Overnight, skies remained clear and winds were light and variable at the near surface, according to surface observations. 


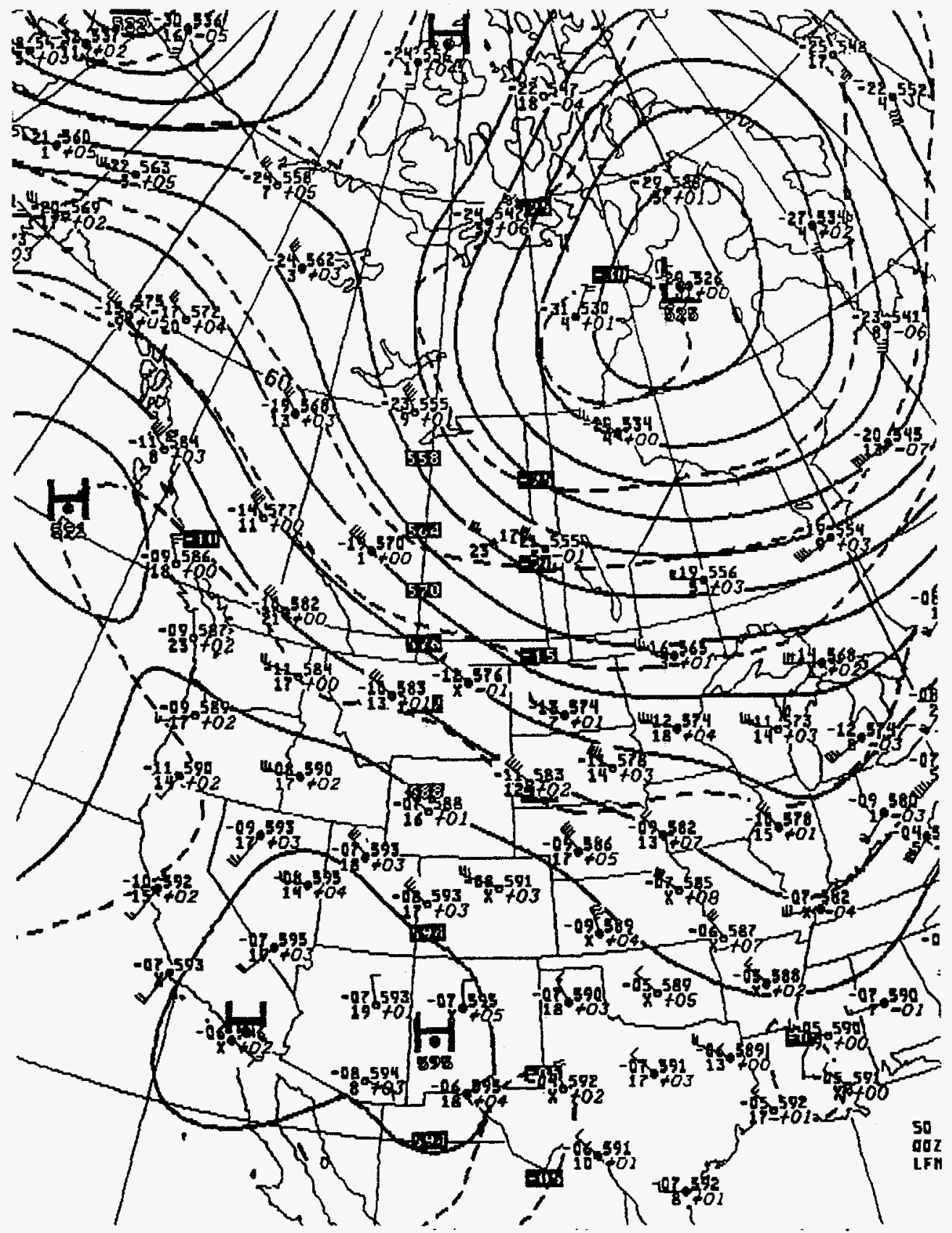

Figure 3.2 Synoptic conditions at $500 \mathrm{mb}$ for a) $00 \mathrm{Z}$, and b) 12Z, 4 Sep 1993. A high to the southwest and low to the northeast of the ASCOT study area create a northwesterly geostrophic flow over the Rocky Mountains. 
The Interaction of Katabatic Winds and Mountain Waves

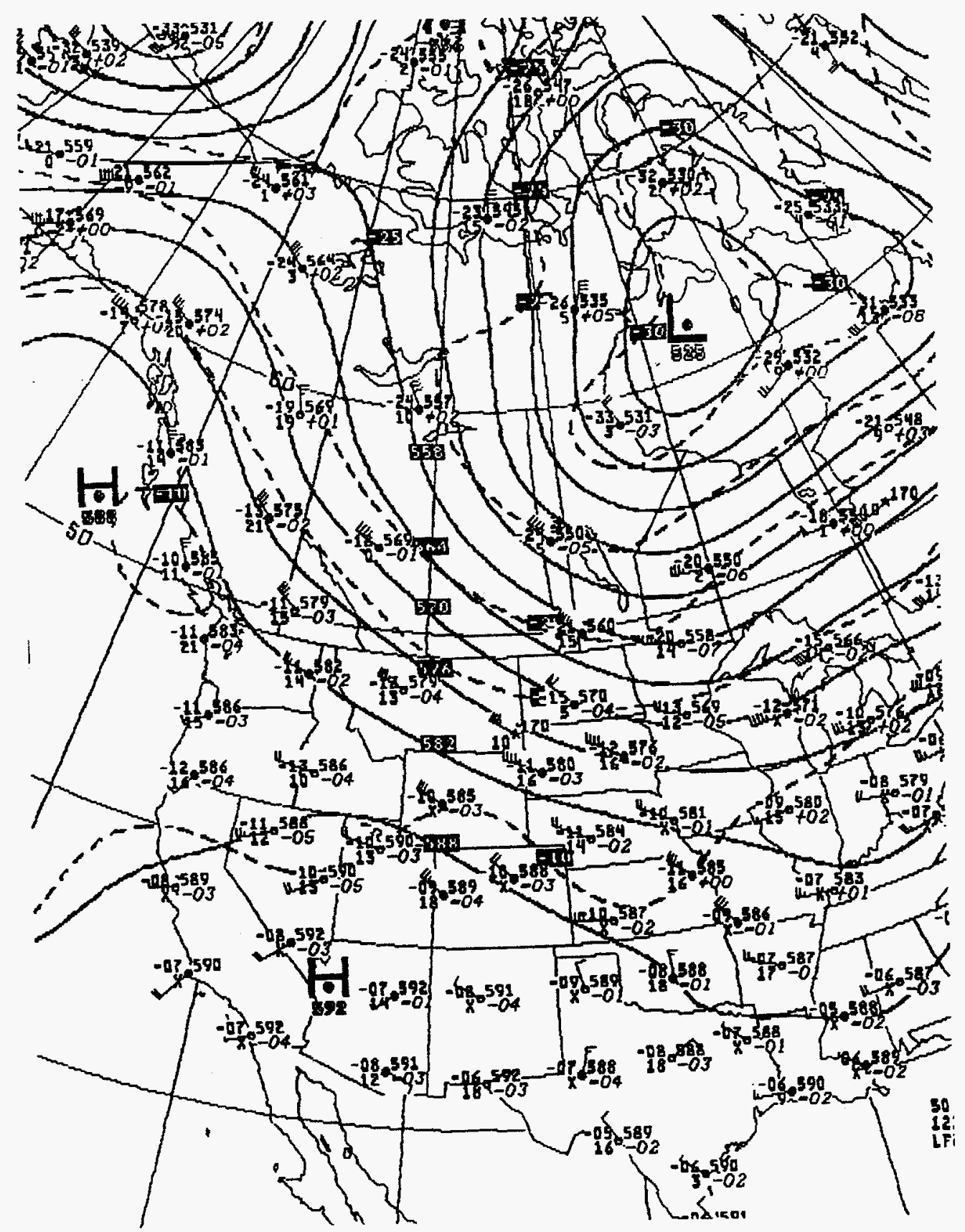

Figure 3.2 Continued. 


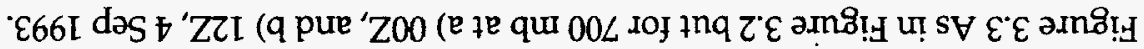

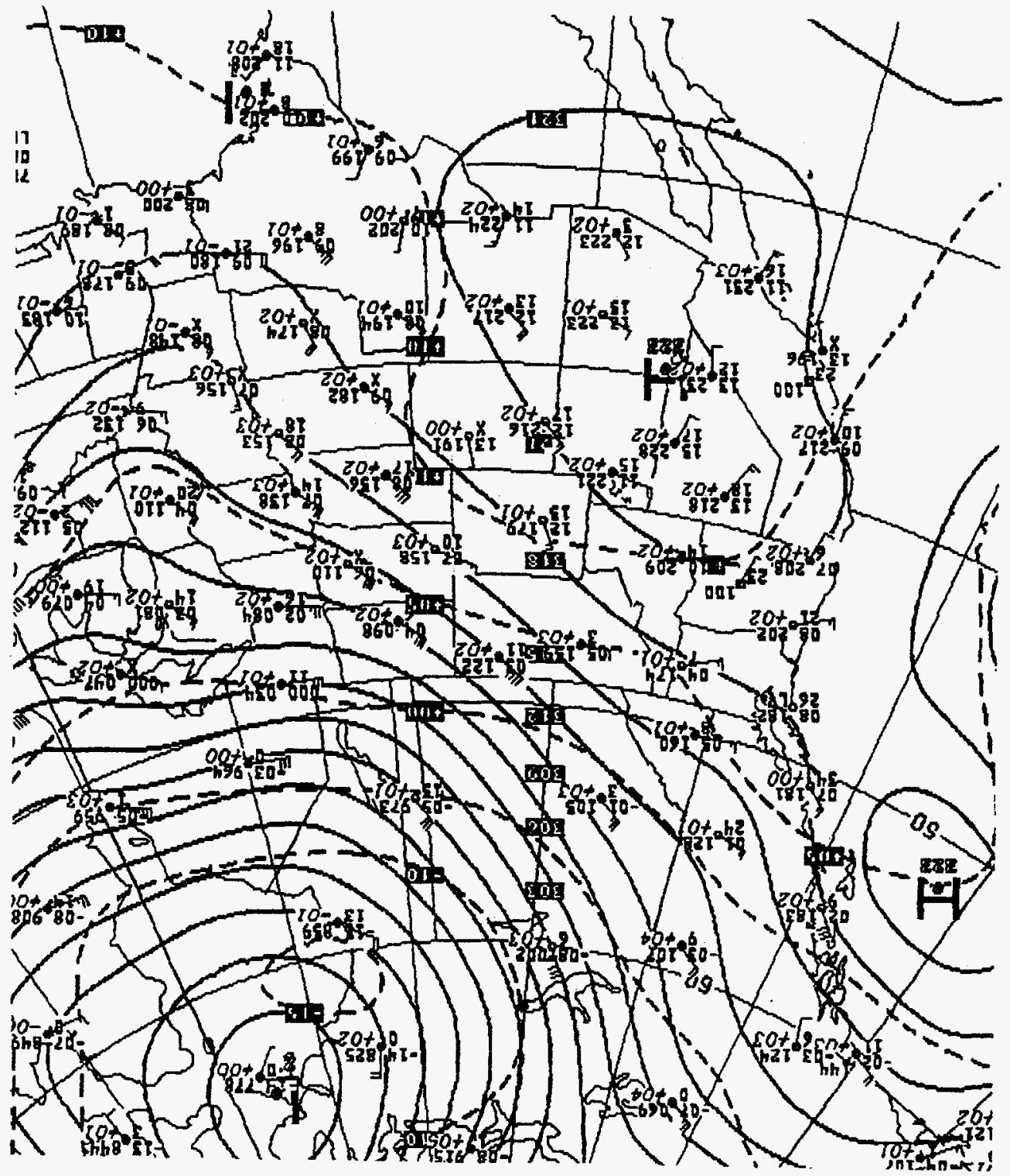




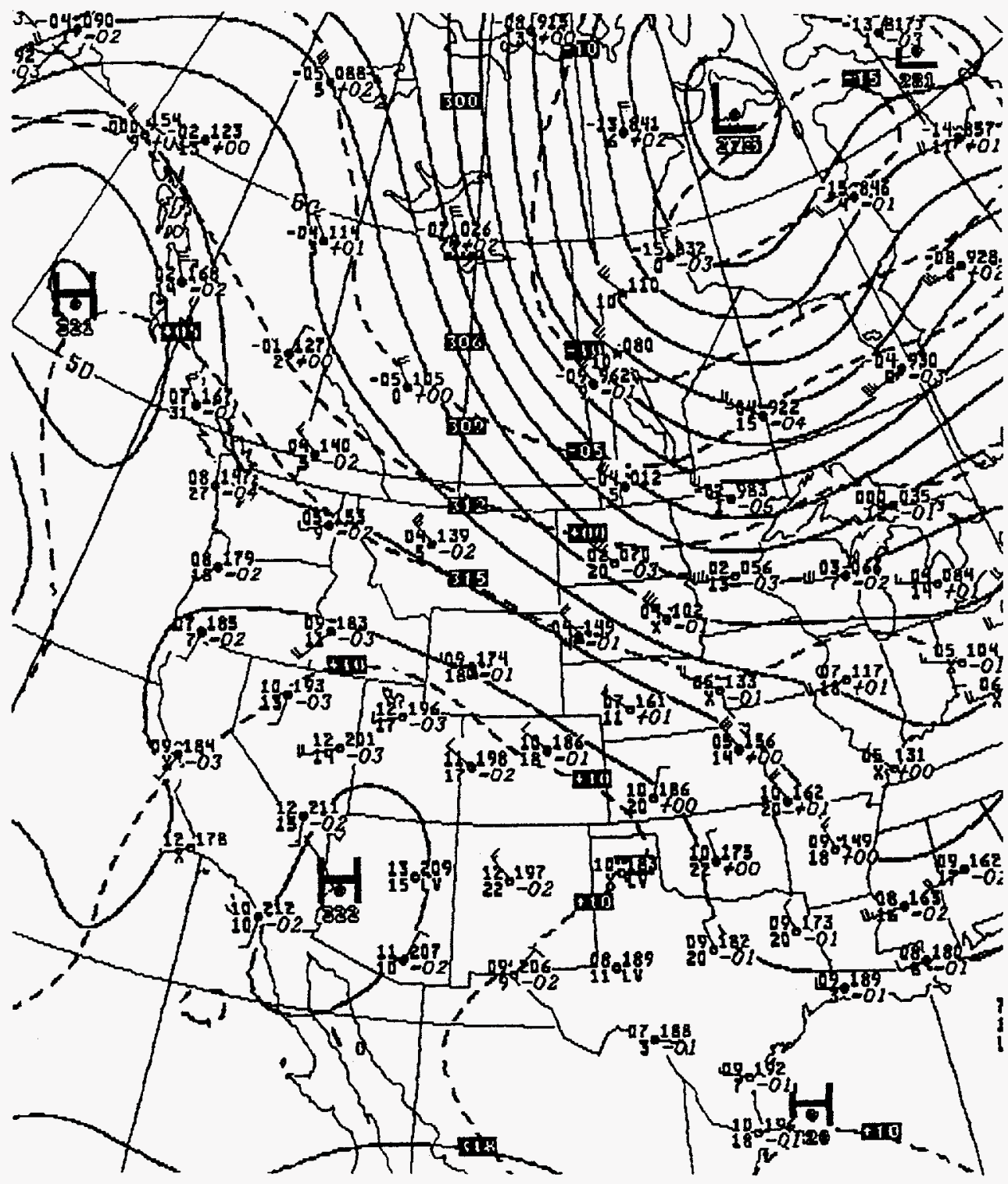

Figure 3.3 Continued. 
Temperatures dropped $\sim 19^{\circ} \mathrm{C}$ to near $11^{\circ} \mathrm{C}$ by morning, indicative of the strong radiative cooling overnight and development conducive to katabatic flow development along the Front Range in areas sheltered from ambient flow. The tower at Rim Rock, in the upper portion of Coal Creek Canyon, indicated that net radiation averaged $-67 \mathrm{~W} \mathrm{~m}^{-2}$ from 1800 - 0600 LST, which was one of the factors causing this night to be ranked 3 rd in the objective rankings; higher than the undisturbed night described in the previous section. Additional details of the overnight development can be found in Appendix A.

The overnight sodar record from the mouth of Coal Creek Canyon verified katabatic flow development, as shown in Figure 3.4, with many of the same features as described for the undisturbed katabatic flow case in the previous two sections. From panel $a$, we note a wind lull both before and after the transition to a katabatic flow of $\sim 165 \mathrm{~m}$ depth with jet structure. A sudden reduction in the $5-7 \mathrm{~m} \mathrm{~s}-1$ wind speed occurs at 0400 LST associated with the intrusion of ambient flow. Given the existence of non-linear mountain wave flow aloft in flow from the northwest, this intrusion could be an indication of particularly strong, temporarily penetrative, rotor activity. From panel $b$, the transition from upslope easterly flow, to downslope, out-canyon flow near $270^{\circ}$, is evident, as well as the transition back to upslope flow toward morning. During the short mountain wave flow intrusion at 0400 LST, winds suddenly shift to an easterly direction, and then return to out-canyon flow with the resumption of katabatic flow. The vertical motion, shown in panel $c$ of Figure 3.4 , corresponds well to the patterns described above, with positive w during upslope and intrusion periods, and subsidence during katabatic flow periods.

\subsubsection{Case 3 - Katabatic flow perturbed by $225^{\circ}$ flow: 14-15 September 1993}

Unlike Case 1, the night of 14-15 September 1993 was chosen for its drainage and what 
appears to be periodic intrusions of upper level air into the drainage flow, presumably a manifestation of mountain wave activity. Though disturbed, ambient conditions were good for katabatic flow development on this night as described in Appendix A. Figure 3.5 shows the height-time profile for this night from the Coal Creek minisodar. In comparison to the relatively undisturbed Case 1 (see Figure 1.2), this case is less well behaved. There appears to be three preferred wind directions; easterly before and after the evening and

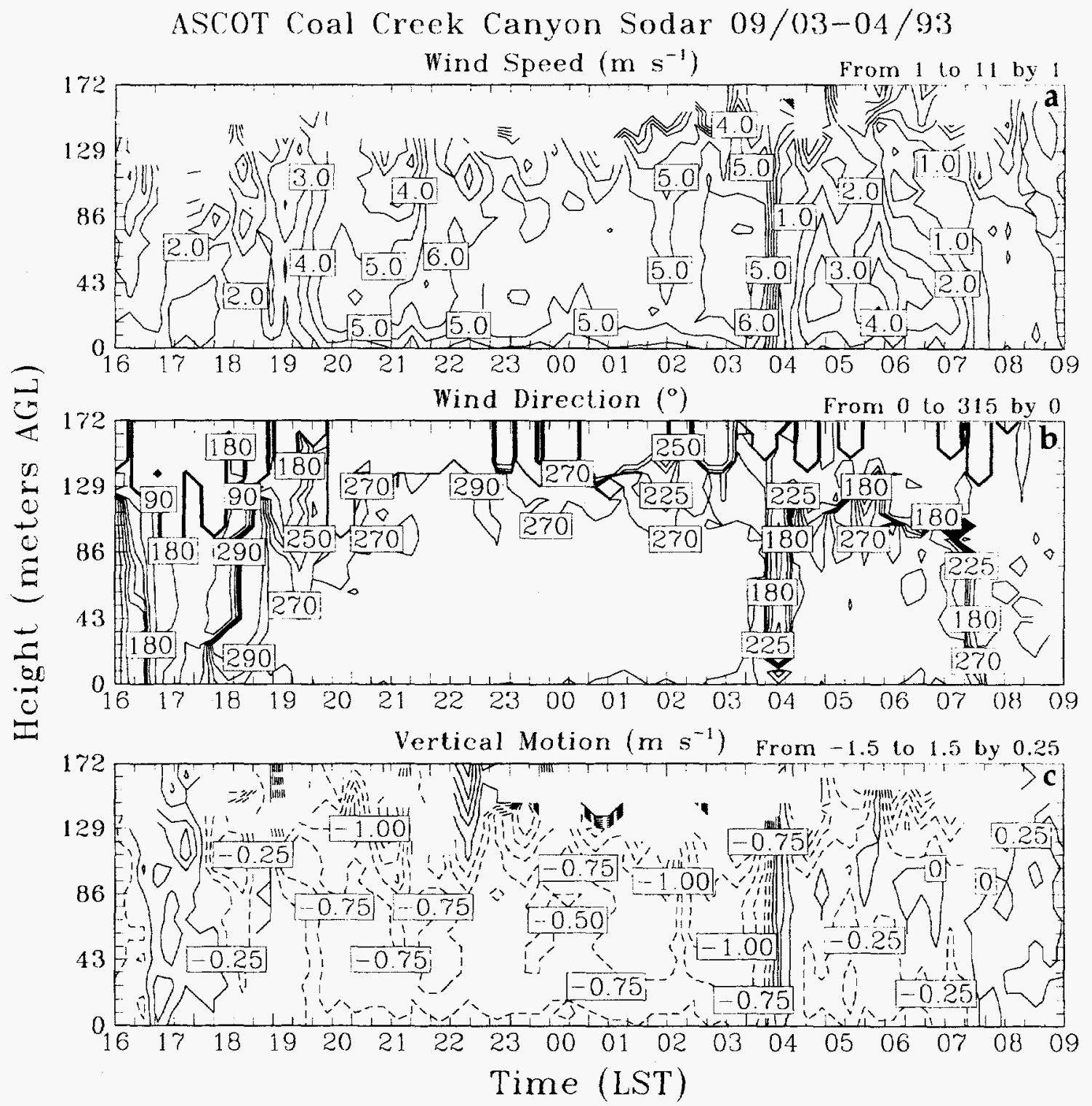

Figure 3.4 Coal Creek Canyon sodar observations from 1600 - 0900 LST for a) wind speed, b) wind direction and c) vertical motion, from the ASCOT 1993 field program for the 3-4 Sep case. Katabatic flow characteristics are clearly shown from the period $1830-0700$ LST. 
morning transitions respectively, westerly when drainage flow dominates and occasional intrusions from southwesterly flow aloft. The two strongest intrusions, indicated by the $240.0^{\circ}$ contour in panel $b$, occur from 2100-2300 LST and 0400-0600 LST and coincide with the abrupt changes to upward vertical motion in the third panel of Figure 3.5. These intrusions are verified by the changes to wind direction for this case night at the $6 \mathrm{~m}$ Rim Rock tower level shown in Table 3.2 (and discussed below)

The occasional intrusions are explained by upper level winds. Analysis of the $2000 \mathrm{~m}$ -
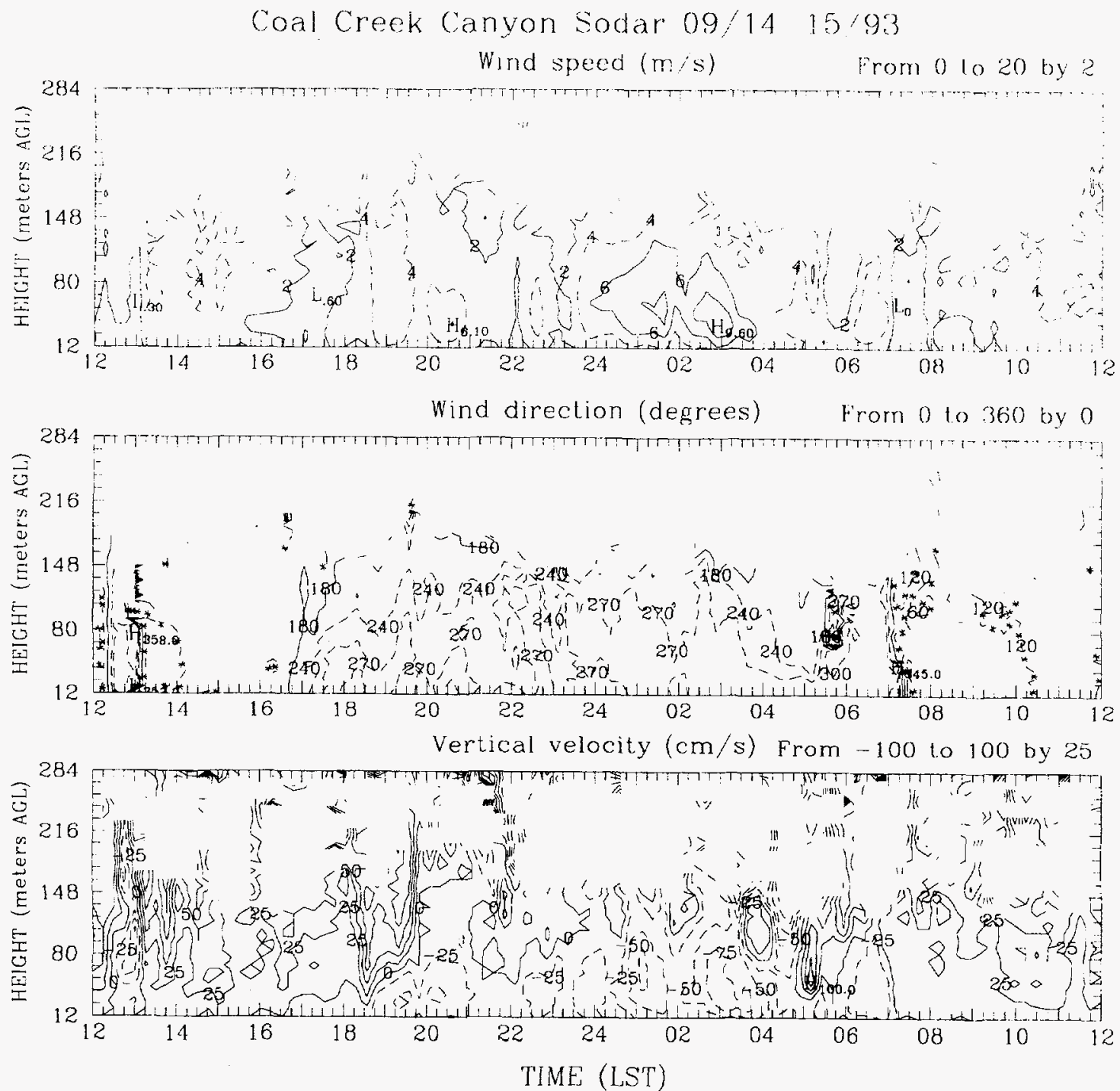

Figure 3.5 ASCOT time-height observations of katabatic flow with mountain wave interaction at the head of Coal Creek Canyon on 14-15 September 1993 by sodar. Panels are a) horizontal wind speed and $b$ ) wind direction and c) vertical motion. Occasional erosion by overlying mountain wave flow is indicated by the periodic changes in wind direction. 
$4000 \mathrm{~m} \mathrm{MSL}$ wind layer based on the Grand Junction sounding indicates an overnight wind direction of $229^{\circ}$. Upper level charts (not shown) indicate that the ambient geostrophic flow direction varied from the WSW to the SW direction by the morning of 15 September (1200Z). Only the analyzed $850 \mathrm{mb}$ geostrophic wind direction was $\mathrm{SW}$ at both $0000 \mathrm{Z}$ and $1200 \mathrm{Z}$. For pressure levels of $850,700,500,300$ and $200 \mathrm{mb}$ the geostrophic gradient would force wind speeds of $10,8,17,22$ and $27 \mathrm{~m} \mathrm{~s}^{-1}$, respectively, which are considerably higher wind speeds than found aloft in Case 1. Given the evidence of increased geostrophic wind speed here compared to Case 1 and the occasional erosion of Case 3 katabatic winds by sodar measured southwesterlies, we can conclude that it was very likely that periodic influence of upper level winds was occurring. Such erosion and its effects on the Coal Creek drainage flow is consistent with ideas documented for Brush Creek Valley, Colorado by Barr and Orgill (1989). It is also consistent that the deeper of the two intrusions is the second one, because winds at all levels had likely turned to the SW direction by this time. Between the two southwesterly intrusions drainage flow is reestablished quite strongly with all the characteristics of undisturbed drainage (albeit only for 6 hours). Thus ambient flow intrusion on draining valleys is supported by both the upper air charts and Coal Creek Canyon sodar measurements.

It is interesting to note that while this night did not make the authors' subjective ranking of the best drainage nights (and should not have because of the intrusions), it is not surprising that such a night appeared in the objective rankings (see boldface in Table 3.1) at number 26. This is because the intrusions did not strongly effect the $6.10 \mathrm{~m}$ tower level on which the objective rankings are based. The reader should note that during the two intrusions ( 2100 LST and $\sim 0500$ LST) that there is a shift in the hour average wind direction 
from westerly component winds to easterly component winds based on the tower data in Table 3.2. This verifies that such intrusions occurred throughout the canyon, but the hour averages of the Rim Rock tower data prevent the intrusions from affecting the objective ranking significantly. The intrusions are, based on the overnight averages in Table 3.1, evident in the high $\sigma_{\theta}$ value of $60.9^{\circ}$ (showing greater wind direction variability relative to the quiescent, top ranked nights). This relatively high value helped prevent this night from ranking objectively higher and supports the use of $\sigma_{\theta}$ as a ranking variable.

Table 3.2: Hour average Rim Rock tower readings for 1800 LST 14 September to 0600 LST 15 September 1993 at $6.10 \mathrm{~m} \mathrm{AGL}$

\begin{tabular}{|c|c|c|c|c|}
\hline Time & Wind & Wind & & \\
\hline Start & Speed & Direction & $\sigma_{\theta}$ & Temperature \\
\hline$(\mathrm{MST})$ & $\left(\mathrm{m} \mathrm{s}^{-1}\right)$ & $\left({ }^{\circ}\right)$ & $\left({ }^{\circ}\right)$ & $\left({ }^{\circ} \mathrm{C}\right)$ \\
\hline \hline 1800 & 0.6 & 328.0 & 86.6 & 5.1 \\
\hline 1900 & 0.7 & 349.0 & 55.5 & 2.9 \\
\hline 2000 & 0.6 & 347.0 & 35.1 & 1.3 \\
\hline 2100 & 0.5 & 16.0 & 46.5 & 0.8 \\
\hline 2200 & 0.3 & 325.0 & 54.3 & 1.0 \\
\hline 2300 & 0.8 & 353.0 & 52.9 & 0.9 \\
\hline 0000 & 1.1 & 352.0 & 23.4 & 0.3 \\
\hline 0100 & 1.0 & 351.0 & 31.4 & -0.7 \\
\hline 0200 & 0.7 & 342.0 & 69.2 & -1.0 \\
\hline 0300 & 0.9 & 314.0 & 95.6 & -0.3 \\
\hline 0400 & 0.7 & 52.0 & 103.2 & -0.6 \\
\hline 0500 & 2.6 & 114.0 & 76.5 & 6.9 \\
\hline 0600 & 2.8 & 31.0 & 76.2 & 9.7 \\
\hline
\end{tabular}

\subsection{Froude Number Climatology, 1966-1993}

Before pursuing the interaction of mountain waves with katabatic flows along the Front Range it is useful to understand the type of flow that typically occurs in the region. Mountain waves have historically been classified by the Froude number or the vertical distribu- 
tion of Scorer parameter, as described in Section 2.6. For this analysis we choose to use the Froude number as it is a more generic representation of flow over an obstacle with which to classify mountain waves. Since the observational data used to represent upstream flow, the Grand Junction twice daily rawinsonde, is only generally representative of flow conditions, the choice of Froude number is appropriate.

Since Froude number is represented by mean thermal and momentum structure with height, as well as barrier height, it is important to select the averaging layer carefully. For these calculations the most appropriate layer to analyze is determined by the local topography east of Grand Junction which rises approximately $2500 \mathrm{~m}$ to the highest peaks along the Continental Divide before descending to approximately $2500 \mathrm{~m}$ to the plains further east. The flow in the lowest $500 \mathrm{~m} \mathrm{AGL}$ around Grand Junction is often stagnant in winter, and strongly influenced by heating in the summer, so that the $2000 \mathrm{~m}$ above this $500 \mathrm{~m}$ deep near surface layer was selected for averaging into the Froude number $(\mathrm{H}=$ barrier height $=2000 \mathrm{~m}$ ). This corresponds to the vertical zone $2000 \mathrm{~m}$ to $4000 \mathrm{~m} \mathrm{MSL}$ for the Grand Junction sounding. A further consideration is that for the canyons on the east side of the Continental Divide that were investigated during ASCOT 1993 only those flows impacting the barrier with a significant westerly component will produce lee side effects. With this in mind, the average wind speed portion of the Froude number calculations were done in two ways, 1) with the total wind, $\mathrm{V}$, but restricted to average wind direction between $235^{\circ}$ and $315^{\circ}$ (a significant westerly component) and 2) with the westerly component only (where easterly flow will give a negative Froude number). Froude numbers calculated using the first method will from now on be termed $\mathrm{Fr}_{\mathrm{v}}$ and those calculated via the second method, $\mathrm{Fr}_{\mathrm{u}}$. Some Froude numbers, calculated with the total wind, $\mathrm{V}$, have 
been already presented in Table 3.1 for each drainage flow night in the objective ranking. A value of zero was assigned to the $\mathrm{Fr}_{\mathrm{v}}$ when it deviated from the given criteria.

\subsubsection{General characteristics}

It was found that $\mathrm{Fr}_{\mathrm{v}}$ was more useful and realistic due to its calculation method. The average value of $\mathrm{Fr}_{u}, 0.31$, was less than half the average $\mathrm{Fr}_{v}$ of 0.63 , because of a low bias inherent in the calculation of $\mathrm{Fr}_{\mathrm{u}}$. This bias is caused by northerly and southerly flow cases whose $\mathrm{u}$ component is typically small leading to a small value of $\mathrm{Fr}_{\mathrm{u}}$. Because of the wind direction sorting used in the calculation of $\mathrm{Fr}_{\mathrm{v}}$ there was no similar effect, particularly because the total wind speed was used. Because of the low bias in $\mathrm{Fr}_{\mathrm{u}}$, it will have a smaller role in the following discussion.

Based on the average Fr values being below 0.8 , it is clear that regardless of which Fr one uses it is clear that near surface air west of the Rocky Mountains from the westerly quadrant is typically unable to surmount the barrier. This is consistent with the study of blocking by Mayr (1993) who found that westerly flow toward the barrier was blocked 50$85 \%$ of the time. It can also be noted that either $\mathrm{Fr}_{\mathrm{u}}$ or $\mathrm{Fr}_{\mathrm{v}}$ indicate that this flow is typically non-linear and will evade analytical solution (Smith 1979). Mayr (1993) also found that blocking was caused by a mesoscale high pressure that forms on the upwind side of the barrier; this pressure may well influence katabatic flow speeds.

Differences between $\mathrm{Fr}_{\mathrm{v}}$ for all the $00 \mathrm{Z}$ soundings and $\mathrm{Fr}_{\mathrm{v}}$ for all the $12 \mathrm{Z}$ soundings were also interesting. The averaged $\mathrm{Fr}_{\mathrm{v}}$ at $00 \mathrm{Z}(0.69)$ was higher than that at $12 \mathrm{Z}(0.53)$ by a considerable margin, although the standard deviation of these values was approximately two-thirds of their magnitude. Analysis of the components of the Froude number, $\theta$ lapse rate $\left(\gamma_{\theta}\right)$ and $\mathrm{V}$, indicate that both factors contribute to an increased $\mathrm{Fr}_{\mathrm{v}}$ at $00 \mathrm{Z}$ (1700 LST); 
$\gamma_{\theta}$ was lower and $\mathrm{V}$ was higher. The explanation for this behavior is simple, during the day surface heating creates a less stable atmosphere that, in turn, allows stronger upper level momentum to mix downward into the sub-barrier height upstream atmosphere. The implication is that a diurnal cycle of mountain wave effects is likely over the mountain barrier, including the canyons of interest to the ASCOT 1993 study.

Overnight it is expected that the mesoscale high pressure on the upwind side of the barrier must strengthen, as would the downwind mesoscale low pressure in response to increasing atmospheric stratification (Queney 1948, Smith 1979). Although the increasing stratification effectively 'shields' developing lee side katabatic flows from shear interaction with mountain wave flow, it also acts to increase the surface pressure perturbation due to the overlying mountain wave as felt at the surface. One can reasonably surmise that at some point in a lee side canyon, depending on actual Fr, a local, mountain wave induced mesolow could at least partially offset local katabatic forcing (cooling induced higher surface pressure). Since the location of the mesolow would change as mountain wave wavelength responded to the evolving atmospheric stratification the location of this effect would also evolve. Such a scenario would certainly induce some changes in katabatic flow with time overnight, perhaps explaining some of the observed variability in these flows. If the lee side mesolow increases in strength (just as the upwind mesohigh increases in strength) in theory this could offset katabatic forcing to a greater extent as the night wears on causing a gradual reduction in katabatic flow speed. The above discussion assumes that upstream flow speed and katabatic forcing are unchanging as atmospheric stratification evolves. The climatological result that the $00 \mathrm{Z}$ sounding has a higher Fr than that at $12 \mathrm{Z}$ occurs regardless of month, season or method of Fr calculation (see Appendix B), 
although this difference minimizes in the winter months.

\subsubsection{Comparison with ASCOT 1993 Froude numbers}

Since the ASCOT experiment focused on August - November of 1993, we are primarily interested in typical Froude number behavior for the Fall period. The Froude number climatology for all months, seasons and years is presented in Appendix B. The Froude number behavior during the ASCOT experiment relative to an average autumn and a typical year gives a useful basis with which to frame our case studies. The last column in Table 3.1 gives the average overnight $\mathrm{Fr}_{\mathrm{v}}$ for each objectively selected case night. Froude numbers here show no particular pattern (note that where $\mathrm{Fr}_{\mathrm{v}}=0.0$, the average wind direction was outside the range $235^{\circ}-315^{\circ}$ ) in magnitude or wind direction and are well within the $\mathrm{Fr}_{\mathrm{v}}$ distribution for all months (Figure B.6) and for the autumn season (Figure B.9). Case night 1 (relatively undisturbed drainage) has a particularly low average $\mathrm{Fr}_{v}$ due to the low average wind speed and high average stability during 02-03 Oct 1993 as might be expected. In contrast the more perturbed case night of 14-15 Sep 1993 had an average wind direction just outside the allowed range at $229^{\circ}$ (giving a $\mathrm{Fr}_{\mathrm{v}}=0.0$ ) and an average wind speed of $6.05 \mathrm{~m} \mathrm{~s}^{-1}$. An average $\mathrm{Fr}_{\mathrm{v}}$ based on these values is 0.51 so that mountain wave scouring of katabatic flow and non-linear effects are more likely on this night than for case night 1 (not considering the dynamic pressure effects of the previous section). Similarly, the case night 3-4 Sep 1993 had a $\mathrm{Fr}_{\mathrm{V}}$ of $\sim 0.45$ overnight, which is also in the non-linear regime.

\subsection{ObServational Contribution to InTERACTION CONCEPT}

From the above case studies it is clear that on certain nights the drainage flows in Coal Creek Canyon are subject to influence by ambient flows. Whereas the relatively undis- 
turbed night of Case 1 developed features typical of 'classic' drainage flows, Cases $2 \& 3$ less consistently showed these features. Case 3 had multiple indications of synoptic scale winds impinging on and mixing with the drainage flow in the canyon, whereas Case 2 showed only one. Intrusions would be manifested as breaks in the strength of the drainage flow or altered wind direction. Although drainage flow was partially eroded, it would regenerate after each intrusion and upper level influence did not prevent the evening and morning transition periods from occurring. This is an indication that while the synoptic flow aloft was sufficient to influence the complex terrain canyon drainage flows of the region, its influence was not strong enough to significantly affect the surface diurnal cycle. Of course, stronger synoptic flow could completely eliminate drainage flows in this canyon for the whole overnight period and reach the surface on the plains as well. Numerous examples of Coal Creek Canyon nighttime flow being completely dominated by ambient flow exist in the sodar data (not shown).

Based on the typical Froude numbers of flow into the Continental Divide it is likely that, in addition to the turbulent interaction of mountain wave flow with katabatic flow, that a dynamic pressure effect occurs. From the review in Chapter 2, we know that a mountain waves causes a surface pressure perturbation that will vary with terrain shape. Thus, pressure changes over a barrier caused by a mountain wave can locally change the pressure gradient force and thereby influence katabatic flow speeds. Also, evolving stratification overnight can cause an evolution of the strength and location of these effects. Modeling studies in upcoming chapters seek to replicate the dynamic pressure interaction mechanism and determine the likelihood of its occurrence. 


\section{CHAPTER 4}

\section{MODEL DESCRIPTION AND VALIDATION}

\subsection{OVERVIEW}

To overcome many of the shortcomings of the observational data set in determining the type of interactions occurring between katabatic and mountain wave flows, the Regional Atmospheric Modeling System (RAMS) was employed. Among the suite of existing mesoscale models in existence in the atmospheric science community RAMS can be argued to be the most versatile; a consequence of over 15 years of development. Its versatility is well-suited to research in atmospheric science on many different scales and in both idealized and realistic modes. The pursuit of information about the interaction of two atmospheric phenomena that operate on vastly different scales further stretches the boundaries of application of RAMS.

Historically, RAMS represents the combined programming efforts of its progenitors Drs. Roger A. Pielke and William R. Cotton of Colorado State University in the early 1970's and innumerable graduate students and research assistants since then. In the early 1980's when Drs. Pielke and Cotton joined their modeling efforts, some of the features of Pielke's hydrostatic models (Pielke, 1974; Mahrer and Pielke, 1977; McNider and Pielke, 1981; McCumber and Pielke, 1981) and Cotton's elastic, non-hydrostatic cloud model (Cotton et al., 1982; Tripoli and Cotton, 1982, 1989) were joined. The modeling effort at 
this time was primarily organized by Dr. Greg Tripoli, much of whose original code remains the backbone of the existing RAMS. When the Pielke and Cotton camps joined together a number of additional features were added to the model, the most notable of which was the terrain-following coordinate system (TFCS). Conceptually, the TFCS is a subset of the generalized coordinate as outlined in Dutton (1976) and Pielke (1984), and with the most specific application to atmospheric modeling, by Gal-Chen and Somerville (1975a, b). The TFCS collapses to the more well-known Cartesian grid over flat terrain but retains many desirable characteristics even over rugged terrain as its name suggests.

Since the early 1980's the sequential addition of numerous features, primarily organized by Dr. Craig Tremback and more recently by Dr. Robert Walko, have made the current version of RAMS, alphanumerically labeled 3a, what it is today. These features include two-way interactive horizontal grid nesting, vertical grid nesting and stretching, data assimilation, a soil model (including moisture and varying soils), a vegetative model (including transpiration for numerous biota) and numerous boundary condition options. Through the appropriate use of these main features RAMS can be adapted to both realistic or idealized simulations with reasonable ease. Note that the most difficult aspect of the modeling is determining what 'appropriate' means for the specific application; selecting inappropriate model options can give poor model results, or worse yet, acceptable model results that are wrong.

In addition to the main mesoscale model, the output from RAMS (called 'analysis files'), can be used further for 1) evaluation of model results in the Analysis Package, or 2) driving a Lagrangian Particle Dispersion Model (LPDM). Analysis files contain standard atmospheric variables, $\mathrm{u}, \mathrm{v}, \mathrm{w}, \theta, \mathrm{r}_{\mathrm{t}}$ and $\pi$, as well as terrain, model grid, microphysical 
(when used), soil and vegetative information, allowing the user to formulate essentially any information he might need to understand the phenomena of interest. The existing analysis package uses NCAR Graphics to produce east-west, north-south, plan view and height-time cross sections, with varying levels of sophistication. A number of features have been added to this package by the author to make its output more useful and understandable by the reader. In addition to improved labeling and contouring information, the package now includes over 70 variables that can be plotted far more quickly than the standard package. Furthermore, in addition to typical contouring, plots can be made in color or grey shade, atmospheric sounding style or as a pixel-fill. The Lagrangian Particle Dispersion Model (LPDM) uses the information in RAMS output analysis files to ascertain the movement of any number of released neutrally buoyant particles and has been described in depth in Poulos and Bossert (1995).

\subsection{RAMS DESCRIPTION}

The model's formulation, as with most atmospheric numerical models, is based on timedependent conservation equations. RAMS can be used in either hydrostatic mode (similar to the Mahrer and Pielke 1977 formulation) or non-hydrostatic mode. The finite difference form of the terms of the various equations can vary greatly depending on the boundary conditions appropriate for the user's particular application and choice of numerical formulation. For the simulations described herein the three-dimensional primitive equations were used in non-hydrostatic, compressible form to ensure adequate physics for the development of mesoscale features.

In tensor notation the compressible form of the three coordinate equations of motion in 
RAMS are (following Tripoli and Cotton, 1982):

$$
\begin{aligned}
\frac{\partial}{\partial t}\left(\rho_{o} \bar{u}_{i}\right)+\frac{1}{a} \frac{\partial}{\partial x_{j}^{*}}\left(a b^{i j} \bar{p}^{\prime}\right)+\rho_{a}^{\prime} g \delta_{i 3} & =-\frac{1}{a}\left[\frac{\partial}{\partial x_{k}^{*}}\left(a b^{j k} \rho_{o} \overline{u_{j} u_{i}}\right)-\bar{u}_{i} \frac{\partial}{\partial x_{k}^{*}}\left(a b^{j k} \rho_{o} \bar{u}_{j}\right)\right] \\
& -\frac{\rho_{o}}{a} \frac{\partial}{\partial x_{k}^{*}}\left(a b^{j k} \overline{u_{i}^{\prime \prime} u_{j}^{\prime \prime}}\right)-\bar{r}_{T} g \delta_{i 3}+\varepsilon_{i j k} f_{i} \bar{u}_{k}^{\prime}
\end{aligned}
$$

where $\mathrm{i}, \mathrm{j}, \mathrm{k}=1,2,3$ and $\mathrm{a}$ and $\mathrm{b}$ are coordinate transformation coefficients inherent in the use of terrain-following coordinates (Clark 1977). In (4.1), $\rho_{0}$ is reference state density, $\bar{u}$ is mean velocity, $\mathrm{p}$ is pressure, $\mathrm{x}^{*}$ is terrain-following coordinate, $\rho_{\mathrm{a}}$ is the density of dry air, $\mathrm{g}$ is gravity, $\delta_{\mathrm{ij}}$ is the Kronecker delta function, $u^{\prime \prime}$ is the sub-grid scale velocity, $\mathrm{r}_{\mathrm{T}}$ is the total water mixing ratio (comprised of vapor, liquid and ice mixing ratio, depending on the complexity of cloud microphysics used), $\varepsilon_{\mathrm{ijk}}$ is the permutation symbol or alternating unit tensor and $\mathrm{f}$ is the Coriolis acceleration. The terms in (4.1) from left-to-right are:

1. local time rate of change of momentum

2. the pressure gradient acceleration

3. gravitational acceleration of dry air

4. the advection of momentum, written as the difference between a flux divergence and momentum divergence term to improve numerical accuracy

5. turbulent momentum flux contribution, parameterized using $\mathrm{K}$ - or eddy viscosity closure (in K-closure turbulent flux is equal to an exchange coefficient, $\mathrm{K}$, multiplied by the gradient of the mean variable)

6. gravitational acceleration due to the density of suspended total water

7. the Coriolis acceleration

In terrain-following coordinates the compressible continuity equation is:

$$
\frac{\partial}{\partial t} \bar{\rho}_{a}{ }^{\prime}+\frac{1}{a} \frac{\partial}{\partial x_{j}^{*}}\left(a b^{i j} \rho_{o} u_{j}\right)=0
$$

Four mass conservation equations for all water variables (not shown) for use in simula- 
tions with extensive cloud microphysics are also formulated. The ninth and final prognostic equation used in RAMS is that for thermodynamic energy:

$$
\begin{array}{r}
\frac{\partial \bar{\theta}_{i l}}{\partial t}=-\frac{1}{\rho_{o} a}\left[\frac{\partial}{\partial x_{k}^{*}}\left(a b^{j k} \rho_{o} \overline{u_{j}} \overline{\theta_{i l}}\right)-\bar{\theta}_{i l} \frac{\partial}{\partial x_{k}^{*}}\left(a b^{j k} \rho_{o} \overline{u_{j}}\right)\right]-\frac{1}{a} \frac{\partial}{\partial x_{k}^{*}}\left(a b^{j k} \overline{\theta_{i l}{ }^{\prime \prime} u_{j}^{\prime \prime}}\right) \\
-\frac{\bar{\theta}_{i l}^{2}}{\bar{\theta}} \frac{\left[L_{l k} P r_{r}+L_{i l^{\prime}}\left(P r_{i}+P r_{g}\right)\right]}{c_{p} T}
\end{array}
$$

where $\theta$ is potential temperature, $\theta_{\mathrm{il}}$ is ice-liquid water potential temperature, $\mathrm{T}$ is temperature, $C_{p}$ is the heat capacity of dry air, $L_{l v}$ and $L_{i v}$ are the latent heats of vaporization and sublimation respectively, and $\operatorname{Pr}_{\mathfrak{r}} \operatorname{Pr}_{\mathfrak{i}}$, and $\operatorname{Prg}$ are the precipitation tendencies of rain water, ice, and graupel, respectively.

The equations described above form a well-posed, closed mathematical set when used with diagnostic relations (not shown, see Tripoli and Cotton, 1982). Numerical integration of this formulation to forecast atmospheric conditions is achieved by the specification of boundary and initial conditions, the use of numerous parameterizations (see Pielke et al., 1992) and time- and space-differencing schemes. For these simulations the upper boundary was specified as a rigid lid where a few of the uppermost model grid levels are used as a Rayleigh friction layer to absorb vertically propagating gravity waves (Clark, 1977). The lateral boundaries were the radiative type of Klemp and Wilhelmson (1978a,b). Initial conditions are described below. Because these model equations are integrated within a grid structure, stable, numerical, time-differencing schemes are used to integrate the equations. The differencing schemes used vary considerably depending on the term differenced. Advective and source terms are time differenced using a hybrid leap frog (for momentum) -forward (for scalars) formulation and Asselin filter; turbulent quantities are 
time differenced using a forward scheme and acoustic terms are time differenced using a forward-backward semi-implicit scheme over a smaller timestep than used for the other terms (due to the high speed of sound waves relative to typical atmospheric motions). In space, derivative terms are differenced with 4th order accuracy in flux conservative form. More complete overviews of RAMS and verification of its accuracy for various meteorological conditions can be found in Tremback et al.(1986), Cotton et al.(1988), Tremback and Walko (1992), Nicholls et al. (1995) and Pielke et al.(1992).

\subsection{The APPlication Of RAMS IN STEEP TOPOGRAPHY}

As expected when modeling mountain waves and katabatic flows in the Rocky Mountains, the possibility of resolving steep slopes arises. Given that RAMS is formulated in a TFCS one would expect this topography to present few problems to the model solutions. However, experience has shown that truncation errors in regions of steep topography due to numerical difficulties at this boundary can cause degraded model results. This then creates a limitation on the slope allowed in a particular model domain with a given $\Delta x, \Delta y$ and $\Delta z$. This and other limits on mesoscale modeling with RAMS in steep topography are discussed below. Note that other non-hydrostatic, limited-area models that use the TFCS but are anelastic claim to be able to simulate slopes that would exceed the limits on RAMS (Gal-Chen and Somerville 1975, Schlunzen 1990). It is unclear at this time whether these claims are true, particularly for simulations of greater than a couple hours in length, since steep slope simulations are poorly documented. Sections below outline the authors attempt to investigate this problem in RAMS.

\subsubsection{Rule of thumb slope maximum criteria}

From the RAMS User's Guide (Tremback and Walko, 1993) one can find clues indicat- 
ing that the completion of simulations containing steep terrain slope(s) could be difficult. Personal communication with Drs. Robert Walko, Craig Tremback and Gregory Tripoli have verified that these limitations exist, with no solution available at this time. The ruleof-thumb that governs this constraint is that the terrain slope for a given $\Delta x$ and $\Delta z$ (or $\Delta y$ and $\Delta z$ ) should not rise more than 3-5 $\Delta z$ across one $\Delta x$. This constraint can be mathematically expressed as,

$$
\alpha_{\max }=\operatorname{atan}\left(\frac{5 \Delta z}{\Delta x}\right)
$$

This formula was developed based on modeling experiences that have shown that as terrain slope increases beyond this steepness the truncation errors in the pressure gradient force calculation become too large and dominate the model solution.

This constraint does not prevent one from simulating extremely steep slope angles, however. At steep slopes, say of $45^{\circ}$, one simply must have horizontal grid spacing approaching that of the vertical grid spacing. In the case where $\Delta z=\Delta x$ a $45^{\circ}$ is easily simulated because the limiting criteria is not violated. However, cases where $\Delta z=\Delta x$ near the ground surface are generally limited to the realm of large-eddy simulation because typical atmospheric calculations with a regional/limited-area model are of much greater horizontal extent than vertical. While a large-eddy simulation may encompass $5 \mathrm{~km}$ in all three coordinate directions with cyclic boundary conditions, typical limited-area domains cannot be cyclic and must encompass domains of 100's - 1000's of kilometers while extending $20 \mathrm{~km}$ vertically. Given that the maximum number of nodes able to be processed on current high-performance workstations is about $10^{6}$ (and this gives very slow performance characteristics), a limited-area domain where $\Delta x=\Delta y=\Delta z=100 \mathrm{~m}$ can be a maximum of an approximately $10 \mathrm{~km}^{3}$ domain. The limiting factor in the above example 
is $\Delta z$ because there is far greater need for resolving atmospheric vertical structure due to the strong gradients in the $\mathrm{z}$-coordinate direction.

Typically then, atmospheric models are run with large aspect ratios (the ratio of $\Delta x$ to $\Delta z$ ). In this way a large horizontal domain can be encompassed while still retaining vertical resolution sufficient to define the atmospheric phenomena of interest. This convenience, however, then puts severe limits on the allowable slope in RAMS, as defined in (4.4). For example, in the case of katabatic flows of $\mathrm{O}(100 \mathrm{~m})$ in depth, resolving vertical structure to a sufficient degree requires $\Delta \mathrm{z} \sim 10 \mathrm{~m}$. In this case, features of $40 \mathrm{~m}$ in size will be sufficiently well handled numerically (Pielke 1984). Further, the horizontal grid spacing must be able to resolve terrain features of significance. In the Front Range the mountain/canyon structure requires a minimum of $500 \mathrm{~m} \Delta \mathrm{x}=\Delta \mathrm{y}$. In this instance, (4.4) suggests a maximum slope of $5.7^{\circ}$ in the terrain $(\tan (\alpha)=0.1)$.

Note that the closer one is to this maximum the more intolerable the noise generated may become. An example of the restrictions (4.4) puts on the modeling domain is found in Poulos and Bossert (1995). They showed that using $\Delta x, \Delta y=500 \mathrm{~m}$ and terrain data from a standard 30 arc-second data set to represent the Front Range will often yield slopes up to $15^{\circ}$. Assuming then that $\Delta \mathrm{z}=10 \mathrm{~m}$ is inviolable to sufficiently capture the katabatic flow of interest, a $15^{\circ}$ slope forces $\Delta x, \Delta y$ to become $187 \mathrm{~m}$ (assuming that you keep terrain slope the same as $\Delta x, \Delta y$ decreases and that maximum terrain slope does not change). For the sake of discussion, let us further assume that numerical errors are tolerable at $\Delta x, \Delta y=$ $200 \mathrm{~m}$ with $\Delta z=10 \mathrm{~m}$ in the Front Range area. In this configuration one then must consider the size of horizontal domain that must be covered in by the grid cells of this size. Ideally for this study that domain must be sufficient to encompass all of Ralston Creek 
Canyon, Coal Creek Canyon and Eldorado Canyon in the north-south direction and from approximately $5 \mathrm{~km}$ east of Rocky Flats to the opposite side of the Continental Divide. This domain size (see Figure 3.1 ) covers $55 \times 40 \mathrm{~km}$, requiring an unreasonable $275 \mathrm{X}$ grid points by $200 \mathrm{Y}$ grid points. No workstation can effectively execute a domain of that size, particularly when one considers that $80-100$ vertical grid points will be needed to reach the stratosphere while simultaneously resolving vertically propagating topographically-induced internal gravity waves. Furthermore, to encompass the scale of the mountain waves accurately within a nudged domain requires an outermost grid scale of over $1000 \mathrm{~km}^{2}$. Obviously, either some sacrifices, model improvements or parallel processing are needed to tackle such a problem, unless a solution is found.

How serious do the improvements to RAMS need to be in order to alleviate this problem? In nature, it is rare for terrain to rise at a $30^{\circ}$ slope for over $500 \mathrm{~m}$ horizontally; this is especially so since the model must smooth out $2 \Delta \mathrm{x}$ terrain features (forcing the $30^{\circ}$ slope to maintain itself for $1000 \mathrm{~m}$ horizontally, a vertical rise of $500 \mathrm{~m}$ ). For real world simulations with $500 \mathrm{~m}$ grid spacing then we can assume that being able to capture a $20^{\circ}$ slope will be sufficient for many complex terrain applications in the Rocky Mountains. This is likely inadequate in the Himalaya or Andes Mountains. In this case the desired allowable terrain rise per $\Delta \mathrm{x}$ can be calculated to be

$$
n=\frac{\tan \left(\alpha_{\text {max }}\right) \Delta x}{\Delta z}=\frac{\tan \left(20^{\circ}\right) \cdot 500 m}{10 m}=18.20
$$

Compared to the desired ratio of $n=5$ from (4.4), a value of $n=18$ seems nearly impossible to achieve. However, a paper by Mahrer (1984) showed a technique that gave improvements in model accuracy of an order of magnitude. An attempt was made at insti- 
tuting Mahrer's (1984) fix into RAMS.

Implementation of the Mahrer technique and subsequent comparison for both zero flow and mountain wave cases indicated a $\sim 20 \%$ improvement over the existing formulation in RAMS regardless of slope, but with a less robust treatment of the lower boundary. This unexpected result suggests that the pressure gradient formulation as it currently exists in RAMS is reasonable in most cases and perhaps the errors arise elsewhere in the numerical formulation. Indeed, since the transform from cartesian to terrain-following coordinates is mathematically exact (Pielke 1984) there should be no difference between the Mahrer (1984) formulation, which simply reverses the TFCS transformation, and the standard TFCS pressure gradient. Unfortunately this conclusion was not reached without considerable coding and evaluation effort. Based on a number of model tests, it was found that if the dynamics of the meteorological phenomenon of interest (i.e. katabatic flow) can be sufficiently resolved by the given grid spacing for the given terrain slope then (4.4) can be violated without serious repercussions for solution quality. The rule-of-thumb in (4.4) typically works because few meteorological phenomena, with some exceptions, can be simulated with a large grid spacing aspect ratio. Successful simulations are shown that violate the rule-of-thumb in upcoming chapters.

\subsection{MODEL PERFormanCE}

The complex phenomenon this dissertation seeks to understand, MKI, is actually the combination of two other phenomena, each of which should be shown to be reproducible in accordance with existing dynamical mechanisms. Therefore, before modeling case studies of katabatic flow/mountain wave interaction, a model verification exercise was undertaken. Since RAMS has shown its basic capability to reproduce basic katabatic flows 
in previous studies (i.e. Mahrer and Pielke 1977 (hydrostatic), Bossert and Poulos 1995) a check on model performance in a mountain wave case was pursued. Toward this purpose a comparison with two-dimensional linear wave theory, similar to that in Clark (1977) was completed. If successful, this comparison lends considerable weight to conclusions made based on later simulations where both mountain waves and katabatic flows are occurring simultaneously.

\subsubsection{Mountain waves: Comparison to linear theory}

Verification of model behavior with respect to linear theory of mountain waves as summarized by Queney (1960) in two-dimensions was used. Should the model fail to reproduce important aspects of linear solutions one may reasonably assume there is 1) an error in the Fortran code, 2) a dynamical mechanism missing in the model or 3) a misrepresentation of the boundary conditions. On the other hand, success in reproducing linear theory solutions does not guarantee future performance during non-linear, idealized or realistic cases, but it is strong proof that the basic formulation of RAMS is sound. In later chapters verification of realistic case studies are compared with actual meteorological data from the ASCOT 1993 program.

For this comparison we use the model configuration tested by Clark (1977) on his nonhydrostatic but incompressible (thereby requiring the solution of a diagnostic pressure equation) terrain-following coordinate model. RAMS was configured as closely as possible to the Clark (1977) experiment with model parameters shown in Table 4.1. Although there are no major differences with the exception of the number of vertical grid points, undoubtedly numerous differences between the formulations of these models, not the least of which are numerical differencing, pressure solver and turbulence parameterization, 
exist. This comparison makes no effort to rectify, quantify or tabulate these differences.

The expectation from linear theory for the above configuration is a simple vertically propagating wave of constant intensity. Figure 4 . I a shows the w field for this solution where in lower regions vertical motion alternates between minima and maxima of approximate magnitude $0.12 \mathrm{~m} \mathrm{~s}^{-1}$. The corresponding solution using RAMS appears in Figure $4.1 \mathrm{~b}$ at $13500 \mathrm{~s}$ into the model simulation where an approximate steady-state had been reached (no true steady-state existed because constant momentum boundary conditions were not enforced). RAMS has reproduced the magnitude and location of the minima and Table 4.1: Model parameters used by Clark (1977) and in this study for comparison with two-dimensional linear theory.

\begin{tabular}{|c|c|c|}
\hline Parameter & Clark & RAMS \\
\hline \hline Horizontal grid spacing $(\Delta \mathrm{x})$ and number & $600 \mathrm{~m}, 62$ & $600 \mathrm{~m}, 62$ \\
\hline Vertical grid spacing $(\Delta \mathrm{z})$ and number & $100 \mathrm{~m}, 82$ & $100 \mathrm{~m}, 122$ \\
\hline Timestep & Variable & $1.0 \mathrm{~s}$ \\
\hline Upper boundary condition (5 level) & Rayleigh friction & Rayleigh friction \\
\hline Lateral boundary condition & Radiative. Kreiss 1966 & $\begin{array}{c}\text { Radiative, Klemp and Wil- } \\
\text { helmson, 1978a }\end{array}$ \\
\hline Witch-of-Agnesi Mountain height & $100 \mathrm{~m}$ & $100 \mathrm{~m}$ \\
\hline Mountain half-width & $3000 \mathrm{~m}$ & $3000 \mathrm{~m}$ \\
\hline $\mathrm{d} \theta / \mathrm{dz}$ & $3 \mathrm{~K} \mathrm{~km}^{-1}$ & $3 \mathrm{~K} \mathrm{~km}^{-1}$ \\
\hline Mean u flow & $4 \mathrm{~m} \mathrm{~s}^{-1}$ & $4 \mathrm{~m} \mathrm{~s}^{-1}$ \\
\hline Smagorinsky Constant, $\mathrm{C}_{\mathrm{s}}$ & 0.25 & 0.25 \\
\hline $\mathrm{K}_{\mathrm{h}} / \mathrm{K}_{\mathrm{m}}$ & 1.0 & 1.0 \\
\hline
\end{tabular}

maxima of vertical velocity quite well compared to the linear solution. The primary differences lie in the greater negative tilt of the $0.0 \mathrm{~m} \mathrm{~s}^{-1}$ contour interval in the RAMS solution and the shape of the vertical velocity lobes which extend beyond the location of those in the linear solution. These differences, though minor, are caused by imperfections in the boundary conditions, non-steady upstream flow and non-linearities inherent in the RAMS 
solution. That is, although the model set-up was chosen such that the linear approximation is valid, i.e. non-linear effects should be minor, there still remains a small non-linear part of the solution.

It is notable that Clark (1977) displays the field of vertical velocity using his model at $4000 \mathrm{~s}$ into the model integration, with considerably less success in reproducing the linear solution than RAMS shows here. It is likely that had Clark analyzed a later time the com-
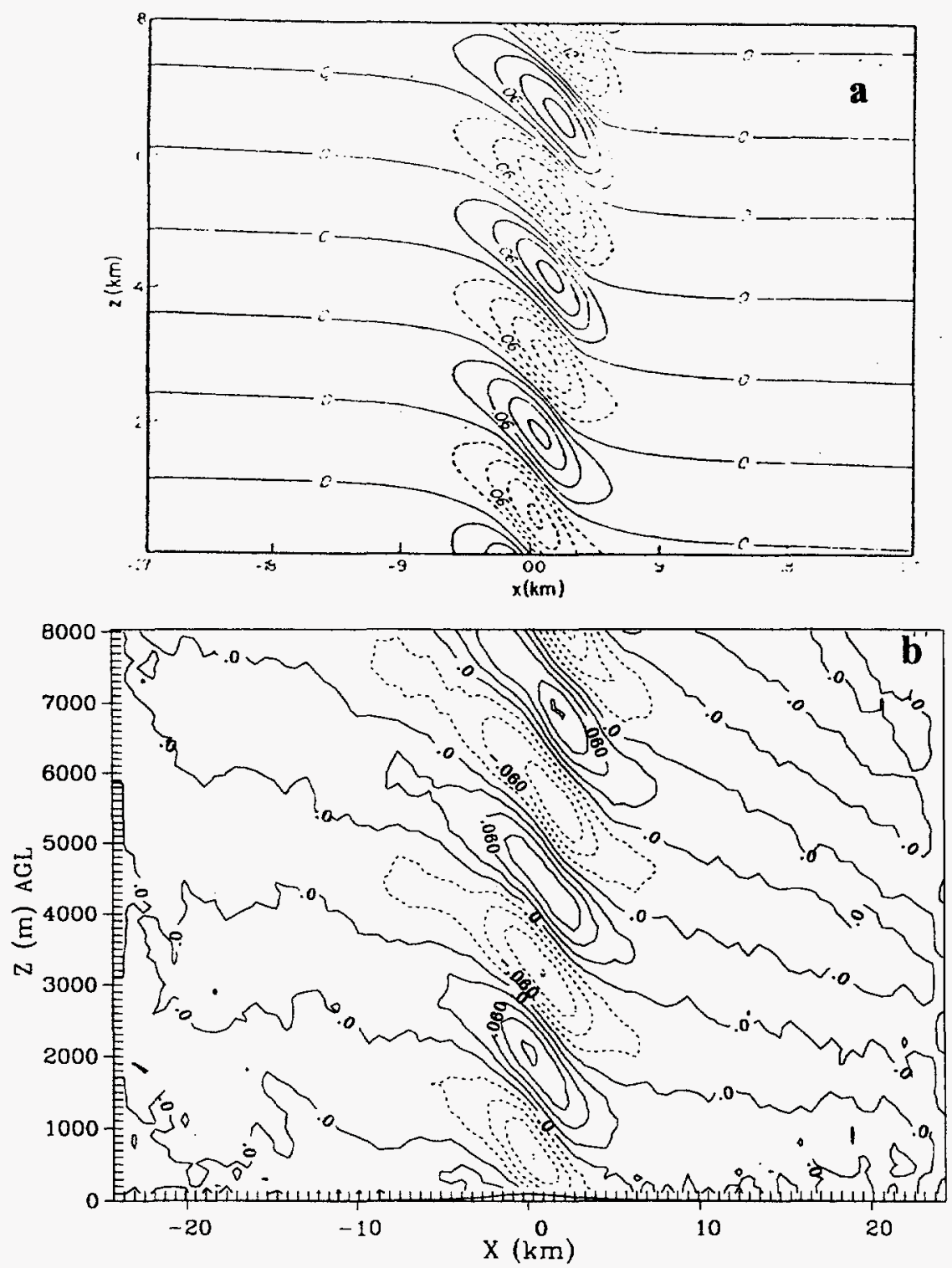

Figure 4.1 Vertical velocity for, a) the steady-state solution of linear mountain wave theory from Clark (1977) and, b) using RAMS at $0.03 \mathrm{~m} \mathrm{~s}^{-1}$ intervals, using the configuration shown in Table 4.1. 
parison would have yielded a closer match. He subscribes his errors to vertical resolution, non-linearities, differences in internal physics between the linear model and his model, the non-linear lower boundary condition and strong eddy mixing. It cannot be ascertained whether these reasons are valid since they are only mentioned briefly by Clark (1977). Although not shown here, a comparison of the RAMS solution at approximately $4000 \mathrm{sec}$ onds to that of Clark (1977, his Figure 1a) indicated that RAMS was able to more accurately reproduce the vertical propagation of the wave as well as its magnitude, but as above had a significantly greater downward tilt of the wave structure than the linear solution. Given sufficient adjustment time RAMS does reproduce expected mountain wave behavior.

A number of different sensitivity studies were also completed. First, the horizontal grid spacing of the model was decreased from $600 \mathrm{~m}$ to $300 \mathrm{~m}$. This alteration garnered little improvement in the quantitative wave structure, but was qualitatively more noisy than the lower resolution solution implying that parameterized turbulent diffusion needed to be higher. Similarly, a second test which decreased the vertical grid spacing to $50 \mathrm{~m}$ from 100 $\mathrm{m}$ simultaneously with the decrease in horizontal resolution as used in the sensitivity test above, showed little quantitative change in the wave structure. This test, too, was more noisy than the original comparison. Two other sensitivity tests which altered the lateral boundary condition showed that the Orlanski boundary condition produces an undesirable noise feature at the lower, outflow boundary, and that the Klemp-Lilly lateral boundary condition performs as well as the Klemp-Wilhelmson boundary condition for this case. The success of the original simulation, which extends to $12 \mathrm{~km}$ (versus $8 \mathrm{~km}$ used by Clark 1977), led the author to select the Klemp-Wilhelmson lateral boundary condition 


\section{Gregory S. Poulos}

and at least a $10 \mathrm{~km}$ deep domain for all future mountain wave simulations with topography and initial flow speed. In cases of zero initial flow or flat terrain the model depth constraint is not as crucial. 


\section{CHAPTER 5}

\section{IDEALIZED Modeling RESUlts}

\subsection{OVERVIEW}

To extract the influence of mountain waves on existing katabatic flows a series of 2-and 3-dimensional simulations were executed. In this idealized 'numerical laboratory' systematic changes in flow characteristics can be most easily extracted, particularly as compared to a rapidly changing realistic situation. Three types of simulations were used to determine the significance of $\mathrm{MKI}, 1$ ) katabatic flow only (referred to as 'KFO', where winds on topography were caused by radiative cooling from an initially zero wind state), 2) mountain wave only (referred to as 'MWO', radiative effects not active) for various Froude number flows and 3) realistic simulations (the combination of 1 and 2 above). Difference fields and direct comparisons of these types of simulations, despite the unavoidable nonlinearities in the problem, give considerable insight into the influence of mountain waves on katabatic flows (Stein and Alpert 1993, also see Section 6.3 on page 161). In all cases the topographic relief was chosen to be $2000 \mathrm{~m}$ and topography was an equilateral barrier in 2- or 3-dimensions. The east and west barrier slopes are $2.5^{\circ}$ which approximates the average slope of the Front Range from the Continental Divide to the plains near Eldorado Canyon. All simulations were run for the overnight period with radiative conditions for 
the autumnal equinox.

\subsection{TWO-DIMENSIONAL SIMULATIONS}

Table 5.1 shows the 9 simulations of varying configuration that were completed. In all cases the horizontal grid spacing was $500 \mathrm{~m}$ while the vertical grid spacing was constant at $20 \mathrm{~m}$ for $500 \mathrm{~m} \mathrm{AGL}$ (to resolve katabatic flows). Above this height the vertical grid spacing sequentially increased by a factor of 1.1 until reaching a fixed size of $400 \mathrm{~m}$ (to resolve the vertical wavelength of mountain waves, see also discussion in Section 6.2). Domain depth was $10.5 \mathrm{~km}$ in the katabatic flow only simulations and $17.7 \mathrm{~km}$ in the mountain wave only and MKI combination runs. The higher domain was necessary for runs with flow over the barrier to adequately capture the vertical propagation of mountain waves.

Table 5.1: A summary of two-dimensional simulations with a $2000 \mathrm{~m}$ mountain.

\begin{tabular}{|c|l|c|}
\hline \multicolumn{1}{|c|}{ Simulation } & \multicolumn{1}{|c|}{ Variations } & Total \# \\
\hline \hline $2.5^{\circ}$ slope & & 1 \\
\hline Katabatic flow only & None & 5 \\
\hline Mountain wave only & Froude \#s $0.25,0.50,0.75,1.00$ & 9 \\
\hline MKI combination & $\begin{array}{l}\text { Froude\# } 0.25,0.50,0.75,1.00 \\
\text { with radiation }\end{array}$ & \\
\hline
\end{tabular}

\subsubsection{Undisturbed katabatic flow}

This simulation investigates the basic katabatic flow that will develop on a slope, $\alpha=$ $2.5^{\circ}$. The domain is oriented in the east-west direction and the only terrain attribute is a mountain centered in the domain of $2000 \mathrm{~m}$ height. The mountain therefore approximates an infinite north-south ridge in this 2-d domain, where its east and west slopes descend at slope $\alpha$ to the flat plains on either side and are mirror images of each other. No nesting techniques were used in these simulations and special care was taken to provide a sufficiently large flat domain on either side of the mountain $(\sim 50 \mathrm{~km})$. The reader should note 
that the initially pointed mountain was smoothed once to ease the severity of the transition from mountain slope to flat plain and round out the mountain peak. Initial winds were zero over the mountainside while the vertical thermal structure was set to $2.5 \mathrm{~K} \mathrm{~km}^{-1}$ in the lowest $3000 \mathrm{~m}$ and $3.4 \mathrm{~K} \mathrm{~km}^{-1}$ (U.S. Standard Atmosphere) above that. The following discussion is sufficient to describe the characteristics of the 3 -d katabatic flow only simulation as well, because it was nearly identical to the 2-d katabatic flow only simulation. In the 3 - $\mathrm{d}$ katabatic flow only simulation there were 21 points in the north-south direction with cyclic boundary conditions at the north-south boundaries, all other qualities being the same as that in the $2-\mathrm{d}$ simulation. Through a deeper understanding of katabatic slope flows a fundamental basis for looking at MKI is established.

From Table 5.2 we see that, as expected, a slopeside katabatic flow grows in strength on each side of the mountain over time. For all but the initial stages, the flow increases in Table 5.2: Katabatic flow details at 8 hours into the 2-d and 3-d, $2.5^{\circ}$ slope, $2000 \mathrm{~m}$ mountain simulations as a function of vertical drop.

\begin{tabular}{|c|c|c|c|c|}
\hline $\begin{array}{c}\text { Vertical Drop } \\
(\mathrm{m})\end{array}$ & $\begin{array}{c}\text { Flow Depth } \\
2-\mathrm{d} / 3-\mathrm{d} \\
(\mathrm{m})\end{array}$ & $\begin{array}{c}\text { Depth } \\
\text { Vertical Drop } \\
2-\mathrm{d} / 3-\mathrm{d}\end{array}$ & $\begin{array}{c}\text { Jet Speed } \\
2-\mathrm{d} / 3-\mathrm{d} \\
\left(\mathrm{m} \mathrm{s}^{-1}\right)\end{array}$ & $\begin{array}{c}\text { Jet Height } \\
2-\mathrm{d} / 3-\mathrm{d} \\
(\mathrm{m})\end{array}$ \\
\hline 250 & $80 / 80$ & $0.32 / 0.32$ & $2.78 / 2.80$ & $30 / 30$ \\
500 & $120 / 120$ & $0.24 / 0.24$ & $6.26 / 6.27$ & $30 / 30$ \\
750 & $120 / 120$ & $0.16 / 0.16$ & $10.51 / 10.52$ & $30 / 30$ \\
1000 & $120 / 120$ & $0.12 / 0.12$ & $15.01 / 15.02$ & $30 / 30$ \\
\hline 1250 & $140 / 140$ & $0.11 / 0.11$ & $19.13 / 19.13$ & $30 / 30$ \\
1500 & $160 / 160$ & $0.11 / 0.11$ & $18.70 / 18.75$ & $30 / 30$ \\
1750 & $220 / 220$ & $0.13 / 0.13$ & $15.90 / 15.87$ & $70 / 70$ \\
2000 & $320 / 320$ & $0.16 / 0.16$ & $14.19 / 14.18$ & $90 / 90$ \\
\hline
\end{tabular}

depth and speed over the upper $70 \%$ of the slope, as shown (Table 5.2) for 8 hours into the simulation. The peak jet speed, $20.5 \mathrm{~m} \mathrm{~s}^{-1}$, occurs at $1454 \mathrm{~m}$ of vertical drop. Within the lowest $\sim 30 \%$ of the slope (last 3 rows of Table 5.2 ), however, jet speed reduces and jet height increases due to upstream effects of the plain. The katabatic flow depth reaches a 


\section{Gregory S. Poulos}

nearly constant 0.11 of vertical drop before the deepening below a vertical drop of 1500 $\mathrm{m}$. As the slope meets the plain (last row of Table 5.2), the jet speed has reduced by $\sim 25 \%$ of its peak value, the jet height has tripled and the total flow depth has doubled. Table 5.2 also contains the values for the 3-d katabatic flow only simulation side-by-side with the data from the 2-d simulation. As small as the differences are, the results from the 2-d simulation will be considered sufficient for describing the 3 -d simulation as well. The flow structure of both the 2-d and 3-d katabatic flow only simulations does not change much after 8 hours.

The katabatic flow behaviour described above is better understood from the vertical cross sections of Figure 5.1, which shows $\theta$ and wind vectors, vertical motion and pressure perturbation for the bottom portion of the east side of the $2.5^{\circ}$ mountain at 8 hours. Strong katabatic winds with a maximum jet over $20 \mathrm{~m} \mathrm{~s}^{-1}$ exist within nearly slope-parallel stable layers. A 1-3 $\mathrm{m} \mathrm{s}^{-1}$ return flow develops aloft, confirming its existence in quiescent, real atmospheric flows as found by Buettner and Thyer (1965) among others. A pool of cold air begins to accumulate on the flat plain, elevating the jet and reducing the wind maximum (see also Bossert and Poulos 1995). The elevation of the jet on the plains results in positive vertical motion over the plain in Figure 5.1b. Internal gravity waves have formed in the middle of the slope pictured, producing alternating upward and downward vertical motion in panel $b$ between $X=29-42 \mathrm{~km}$ (see also the potential temperature undulations in panel $a$ ). From panel $c$, we note that this katabatic flow was caused by the mechanism described in Chapter 2, a horizontal pressure gradient away from the barrier induced by cooling. Further, panel $c$ shows that katabatic flow speed reduction approaching the plain is caused by the pressure gradient forcing from the plain towards the moun- 


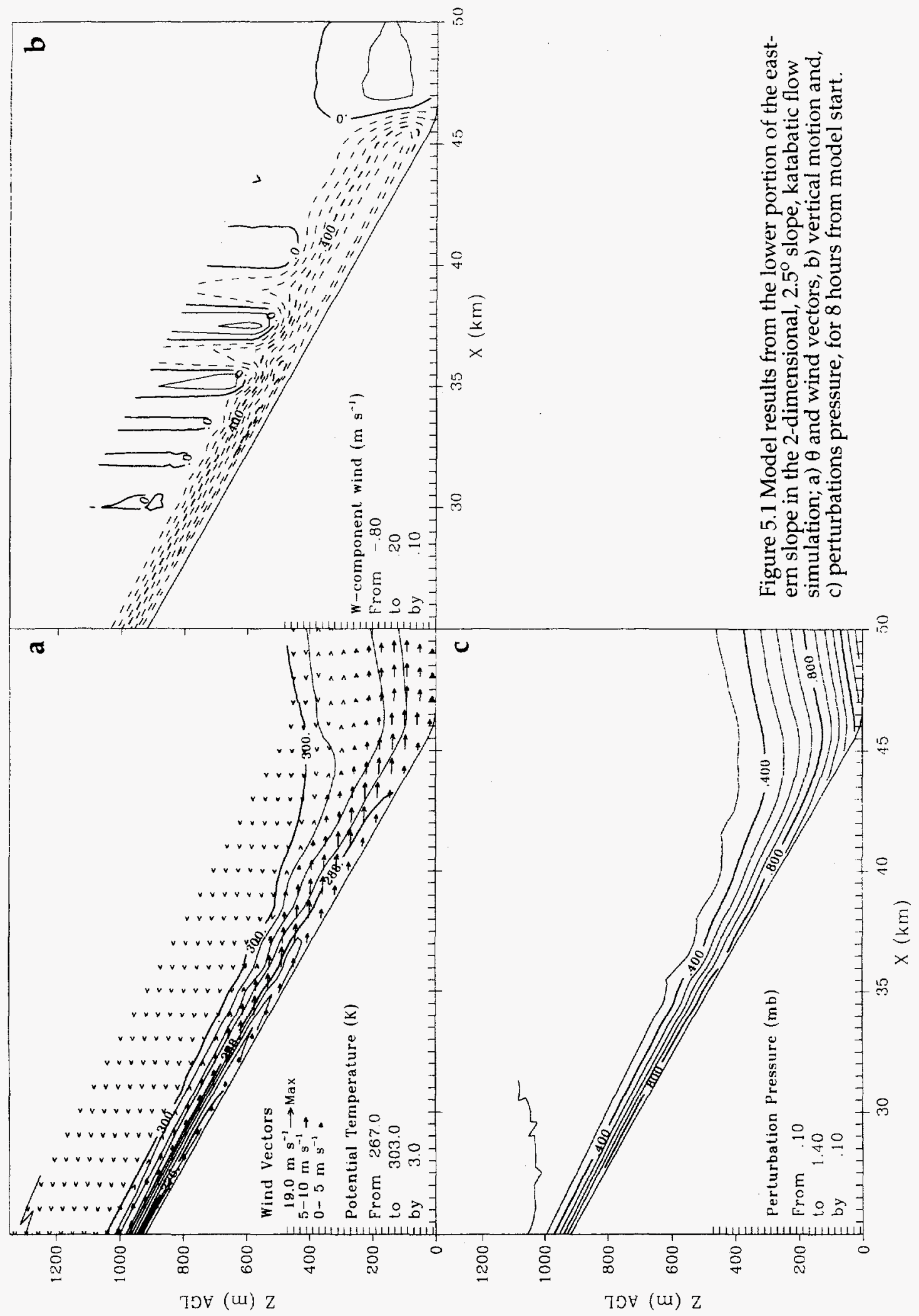


tain. The low-level inversion strength is $\sim 80 \mathrm{~K} \mathrm{~km}^{-1}$, which, although it seems high, was regularly observed by meteorological towers across a vertical distance of tens of meters during the ASCOT experimental program.

A detailed perspective on the dynamics operating in this modeled katabatic slope flow can be developed from high time resolution data, such as the two minute interval data shown in Figures 5.2 and 5.3. These Figures are for a point mid-slope in the center of the north-south domain on the east side of the $2.5^{\circ}, 3$-d north-south ridge. Furthermore, whereas Figure 5.2 shows data for a continuous number of vertical grid points on each panel, Figure 5.3 only contains data for the 3 lowest vertical grid points, $10 \mathrm{~m}$ AGL, $30 \mathrm{~m}$ AGL (jet height) and $50 \mathrm{~m} \mathrm{AGL}$, at this horizontal location. Three stages of katabatic flow development are labeled in the Figures. The reader should note that the Coriolis force was active, but insignificant, throughout these simulations, and therefore is not plotted directly (although it would show up in the residual if significant).

From panels $a$ and $d$ of Figure 5.2 we note that, although longwave radiational cooling commences immediately, it is not until $\sim 30$ minutes that a 120 m layer of katabatic flow, without jet structure, begins to develop in response to the pressure gradient force (PGF). This PGF is of such small magnitude that it is hardly noticeable on Figure 5.3. Panel $d$ shows that, with sufficiently high vertical resolution, radiative flux divergence of the magnitude observed by Manins (1992, $15-20 \mathrm{~K} \mathrm{day}^{-1}$ ) can be simulated. During Stage 1 advection is insignificant (Figure $5.3 a-c$ ) but some negative turbulent forcing on $\mathrm{u}$ exists late in the stage (see arrow labeled ' $T$ '). Simultaneous with the onset of minor turbulent forcing, stable layers begin to grow in earnest as can be seen in Figure5.2c, as cool surface air is transferred to higher altitudes. The PGF increases rapidly throughout the latter part 


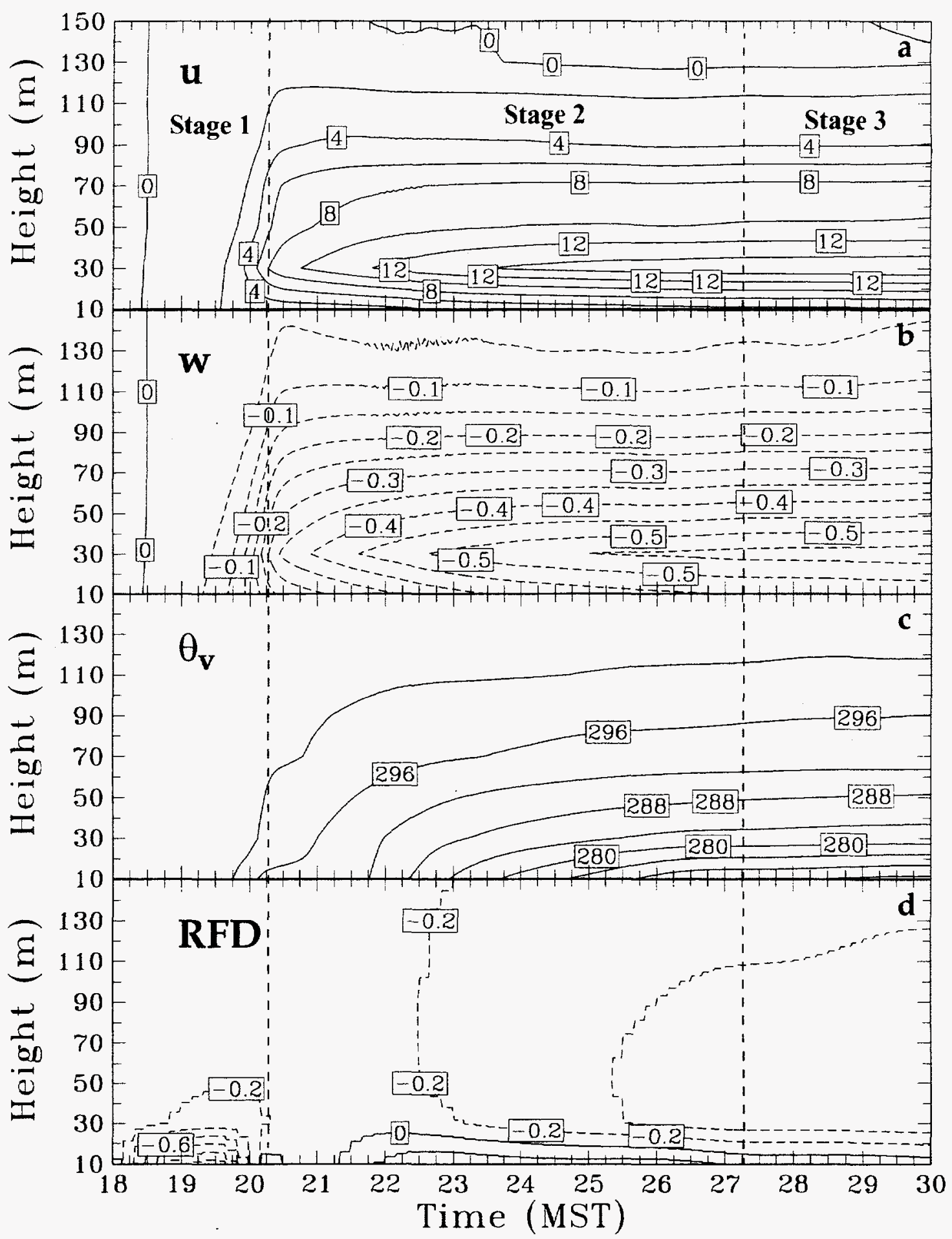

Figure 5.2 Time-height plots halfway down the eastern slope of the 3-dimensional, $2.5^{\circ}$ slope katabatic flow only simulation for a) $\mathrm{u}$-component wind speed in $\mathrm{m} \mathrm{s}^{-1}$, b) vertical motion in $\mathrm{m}$ $\mathrm{s}^{-1}, \mathrm{c}$ ) virtual potential temperature in $\mathrm{K}$ and, $\mathrm{d}$ ) radiative flux divergence in ${ }^{\circ} \mathrm{C} \mathrm{hr}{ }^{-1}$. Data is plotted with 2 minute intervals. 
of Stage 1, despite negative turbulent forcing that mixes upward the cold air responsible for the PGF. Throughout the lowest $150 \mathrm{~m}$ vertical motion is negative, as expected for this katabatic flow (Figure 5.2b).

Entering Stage 2, a number of changes occur. First and foremost, katabatic flow jet structure develops (see Figure 5.2a). Although cooling and therefore PGF is greater at the lower grid level (10 m AGL, see Figure 5.3c), wind speed is weaker than at $30 \mathrm{~m} \mathrm{AGL}$ due to greater frictional retardation. Note in Figure $5.3 \mathrm{a}$, that at $50 \mathrm{~m}$ AGL the turbulent forcing is even further reduced from that of $30 \mathrm{~m} \mathrm{AGL}$ but the wind speed there is not stronger than that at $30 \mathrm{~m} \mathrm{AGL}$. This is because cooling at $50 \mathrm{~m}$ AGL is sufficiently less than that at $30 \mathrm{~m} \mathrm{AGL}$ so as to reduce the PGF, and create an ultimate summation of positive $u$ tendency less than that at $30 \mathrm{~m} \mathrm{AGL}$. Also, advective forcing begins in earnest at the start of Stage 2, just as the total acceleration peaks (dashed arrow labeled ' $A$ ' in Figure $5.3 b)$ at jet height. A clear dynamic effect occurs when advection becomes significant deceleration. Therefore, advection plays a key role in keeping katabatic flow speeds bounded above the surface layer, where turbulent effects are reduced. Note that prior to the onset of deceleration due to advection, that turbulent forcing alone was not sufficient to stop the growth of the $u$ acceleration (surging) in Figure $5.3 \mathrm{a}$ and $\mathrm{b}$, whereas for the $\mathrm{u}$ component in Figure 5.3c, which feels the effect of the surface layer parameterization, pressure gradient is balanced by turbulent forcing alone (categorized as equilibrium flow by Mahrt 1982).

At the same time as negative advective forcing starts in Stage 2, the negative potential temperature tendency reaches it maximum cooling rate of $4.0 \times 10^{-3} \mathrm{~K} \mathrm{~s}^{-1}\left(-14.4 \mathrm{~K} \mathrm{hr}^{-1} !\right)$, although only for a few minutes (not shown). This cooling at jet height is primarily forced 
by turbulent mixing of cold surface air upwards while RFD accounts for $\sim 2 \%$ of the cooling and is partially offset by advective warming (potentially warmer air at higher alongslope mountain altitudes is advected along-slope). The atmosphere responds to this rapid cooling by increasing katabatic flow and advecting the cold air downslope. We have therefore found a mechanism by which the characteristic structure of katabatic flow - an increase in katabatic flow speed with distance downslope - can develop. The cold air traveling down slope, rather than reaching equilibrium through adiabatic warming, is continually cooled by RFD and turbulent mixing. Thus, a positive PGF force is maintained and flow speed continues to increase in the downslope direction. Eventually, however, flow speeds could become great enough that adiabatic warming is sufficient to overcome RFD and turbulent cooling so that a true thermal balance is achieved and downslope acceleration ceases. Throughout the remainder of Stage 2, katabatic flow speed and stability increase while temperatures decrease. It should also be noted that RFD near the surface actually becomes slightly positive in the lowest layer, compared with the strong negative RFD in Stage 1.

Stage 3 delineates the period of time where conditions are very close to steady state. We can see from Figure 5.2 that fields of $\mathrm{u}, \mathrm{w}, \theta_{\mathrm{v}}$ and RFD are relatively steady compared with either Stage 1 or Stage 2. This relative steadiness is best verified from Figure 5.3 for the 3 levels 10,30 and $50 \mathrm{~m} \mathrm{AGL,} \mathrm{where} \mathrm{the} \mathrm{total} \mathrm{tendency} \mathrm{approaches} \mathrm{zero} \mathrm{and} \mathrm{the} \mathrm{mag-}$ nitude of the various tendencies pictured is nearly unchanged. By the beginning of Stage 3 a near balance of temperature forcing is established (not shown) so that cooling occurs at a slow pace (governed primarily by near surface RFD rates of -0.2 to $-0.4 \mathrm{~K} \mathrm{hr}^{-1}$ similar to the temperature balance found by van den Broeke et al. (1994). The slight cooling that 


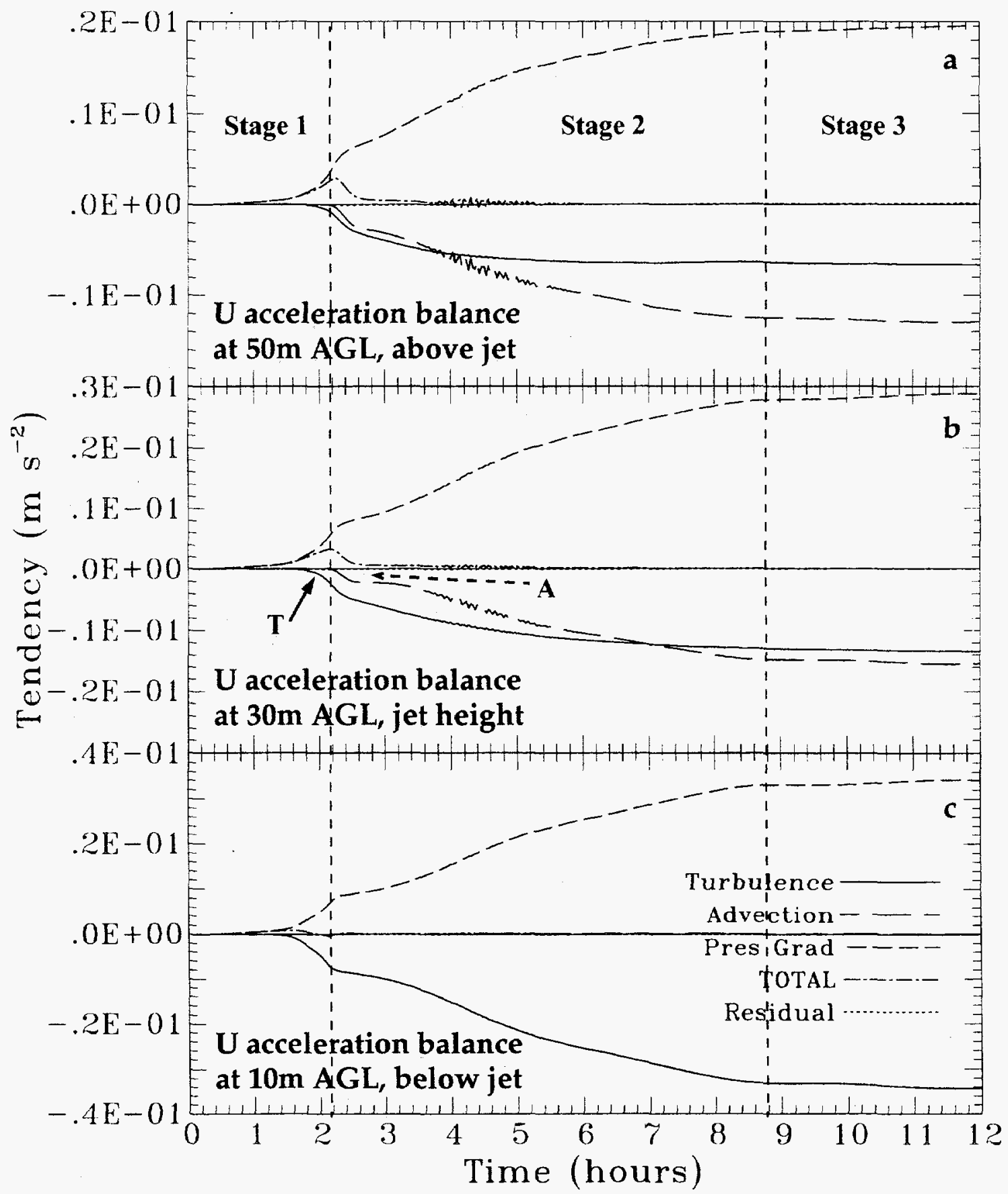

Figure 5.3 The acceleration balance of $u$ component wind for the 3-d, $2.5^{\circ}$, katabatic flow only simulation at a) $50 \mathrm{~m}$ AGL, above the jet, b) $30 \mathrm{~m} \mathrm{AGL}$, jet height and, c) $10 \mathrm{~m}$ AGL, below jet height. This location is halfway down the eastern slope of the $2000 \mathrm{~m}$ tall mountain. Three stages of katabatic flow development are labeled as are the onset of negative turbulent (' $T$ ') and advective forcing (' $\mathrm{A}$ ') at jet height. Data is in 2 minute intervals. 
does occur causes a small $\mathbf{u}$ acceleration throughout the rest of Stage 3 to the end of the simulation. Using the nomenclature of Mahrt (1982) as described in Chapter 2, the flow shown in Figure 5.3c at $10 \mathrm{~m}$ AGL is 'equilibrium flow' and that of the other two panels at higher altitudes are 'shooting flow'. Consequently, the katabatic flow in this simulation cannot be generally classified as a single type. In the bulk form used by Mahrt (1982), the flow at this point is a shooting flow, however, because pressure gradient is balanced by turbulence and advective forcing on average through the katabatic layer.

There is another feature in Figures 5.1-5.3 that deserves mention. Figure 5.1 contains obvious gravity waves in all of its panels within and near the top of the katabatic flow layer (as distinct from the return flow). These waves are shown by wave-like undulations in the potential temperature field, alternating upward and downward motion and waves of perturbation pressure in panels $a-c$, respectively. The cause for this behaviour is that simultaneous with the first appearance of these waves at $\sim 3.5$ hours into the simulation, is the development of significant (i.e. $1-2 \mathrm{~m} \mathrm{~s}^{-1}$ ) return flow above the strengthening katabatic flow. Thus, the strong shear between the return flow and katabatic flow may, just then, become sufficient to generate a dynamically unstable atmosphere. In such a case, we expect gravity waves to form as described by Thorpe (1969, 1973a, b) and Woods (1969). These gravity waves then exist throughout the remainder of the simulation though they are most favored at the bottom $1 / 3$ of the mountain slope. Though the timing of the development of return flow aloft coincides with the wave development, return flow is not a requirement for dynamic instability. The gravity waves are the cause of the high time resolution variability seen in Figures 5.2 (panels $a$ and $b$ ) and 5.3 (panels $a$ and $b$ ) between 3.5 and 5.5 hours into the simulation. In Figure $5.3 \mathrm{a}$ and $\mathrm{b}$, this variability is generated 
within advective forcing, and reflected in the total forcing, though it does not appear in panel $c$. The period over which these fine scale undulations occur, corresponds to that 2 hour period during which gravity wave structure can be noted over the horizontal position of the data shown in these Figures (halfway down the eastern slope of the mountain).

Simulations of katabatic flow with slopes greater/(less) than $2.5^{\circ}$ (i.e. $1^{\circ}, 5^{\circ}$ and $10^{\circ}$ ), not discussed in depth here, went through the 3 stages of katabatic flow development described above at a faster/(slower) pace. Changing the initial thermal stability to greater or less than that described here, also does not eliminate any of these stages, although it does alter their timing. At steeper slope angles the gravity waves described in the previous paragraph were sufficiently unstable so as to break into Kelvin-Helmholtz waves. One of the primary internal mechanisms that generates katabatic flow variability is therefore selfgenerated gravity waves.

\subsubsection{Mountain wave only}

A series of simulations with varying Froude number, but without radiative cooling, were also completed. The purpose of these simulations was to define the atmospheric response to a variety of idealized, but realistic, flows over topography. In all cases, static stability was initialized to $2.5 \mathrm{~K} \mathrm{~km}^{-1}$, as in the katabatic flow only simulations, to $1000 \mathrm{~m}$ above mountain top. Using this stratification to calculate Brunt-Vaisala frequency, and a barrier height of $1500 \mathrm{~m}$, Fr was defined by setting the wind speed to the appropriate value. A value of $1500 \mathrm{~m}$ for barrier height was used, rather than $2000 \mathrm{~m}$, because wind speed was prescribed to be 0.0 at the surface with a linear increase to the selected speed at $500 \mathrm{~m}$ above the lowest topography, and constant above that. The reason this was done was to be consistent in the comparisons with the MKI runs. In the MKI idealized simula- 
tions the katabatic flow developed to a depth of $500 \mathrm{~m}$, and, so as not to unduly effect the katabatic flow, winds were not-spun-up in the lowest $500 \mathrm{~m}$ above the lowest topography. Each simulation lasted 12 hours.

Each simulation behaved as expected, with strongly accelerated flow on the eastern slope, as depicted in Figure 5.4. Compared with their initialized wind speeds of $3.39 \mathrm{~m} \mathrm{~s}^{-1}, 6.78$ $\mathrm{m} \mathrm{s}^{-1}, 10.17 \mathrm{~m} \mathrm{~s}^{-1}$ and $13.55 \mathrm{~m} \mathrm{~s}^{-1}$, respectively, the $\mathrm{Fr}=0.25,0.50,0.75$ and 1.0 simulations accelerate by a maximum factor of $3.24,2.65,2.55$ and 2.21 , respectively, on the eastern slope by 3 hours. Due to their weaker forcing, successively smaller Fr mountain waves descend less far down the lee side of the mountain (or separate from the mountainside nearer the top). In addition to strong vertical perturbations, the $\mathrm{Fr}=0.25$ simulation is seen to have strong low-level return flow and an elevated wave breaking region with little lee wave structure. Also in the non-linear regime, the $\mathrm{Fr}=0.50$ simulation contains breaking waves and lee waves downstream of the barrier. The $\mathrm{Fr}=0.75$ simulation contains weak wave breaking aloft but the $\mathrm{Fr}=1.00$ does not, whereas strong lee waves exist in both. The $\mathrm{Fr}=0.75$ simulation has return flow at the lowest levels, but the $\mathrm{Fr}=1.00$ simulation, where the only negative contours on the eastern side are under the lee waves, instead shows low-level rotor activity. On the western side of the barrier, negative u contours in the $\mathrm{Fr}=0.25$ and $\mathrm{Fr}=0.50$ plots show that blocked flow has developed at the lowest levels, as expected. Blocking is also very weakly occurring in the $\mathrm{Fr}=0.75$ simulation beneath the 0.0 contour, though no negative contours are present on the western slope. Blocking does not occur in the $\mathrm{Fr}=1.00$ simulation as expected from theory (see Figure $5.4 d)$

The deformation of the initially horizontal potential temperature profile is shown for 
Gregory S. Poulos

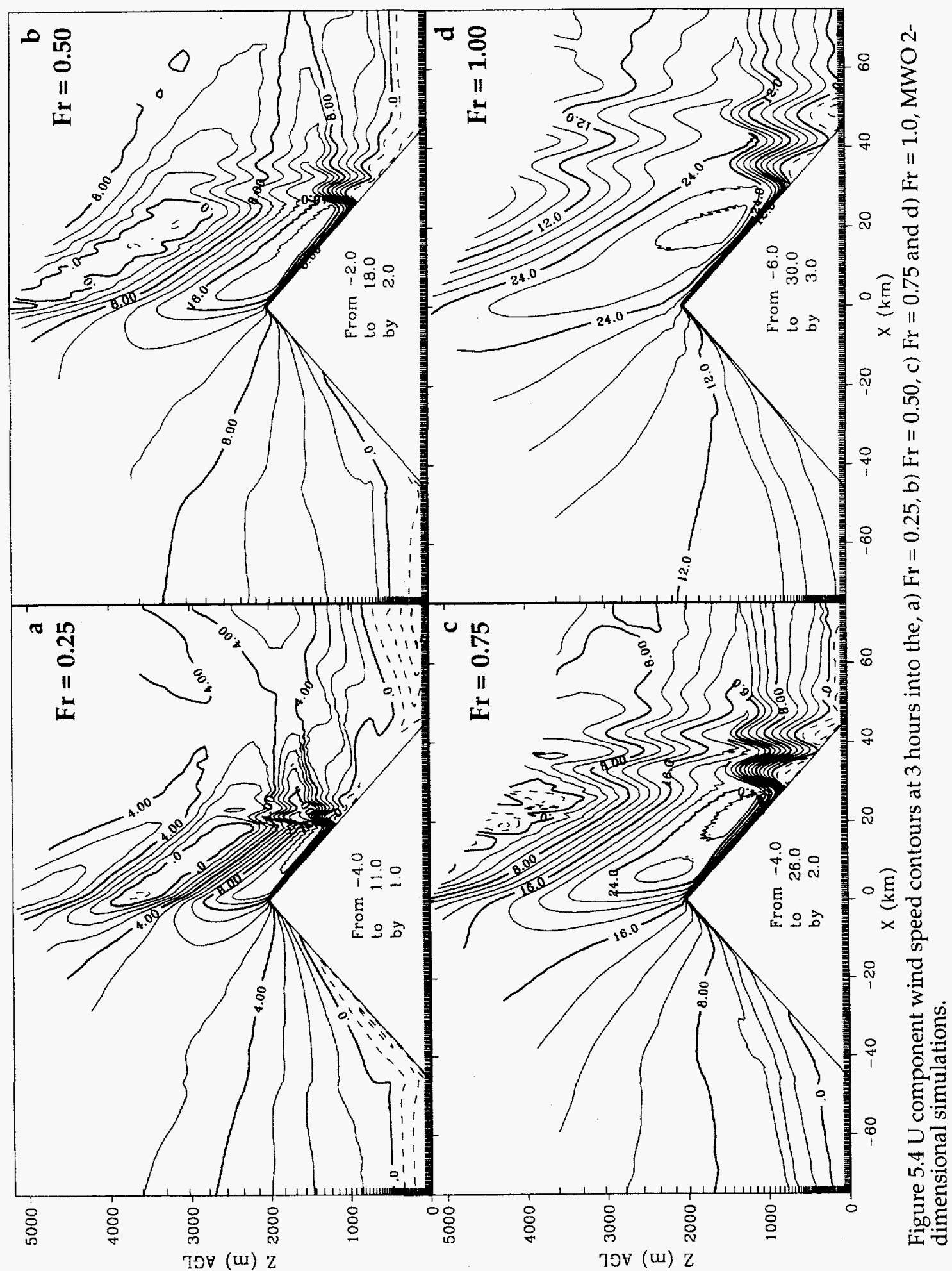




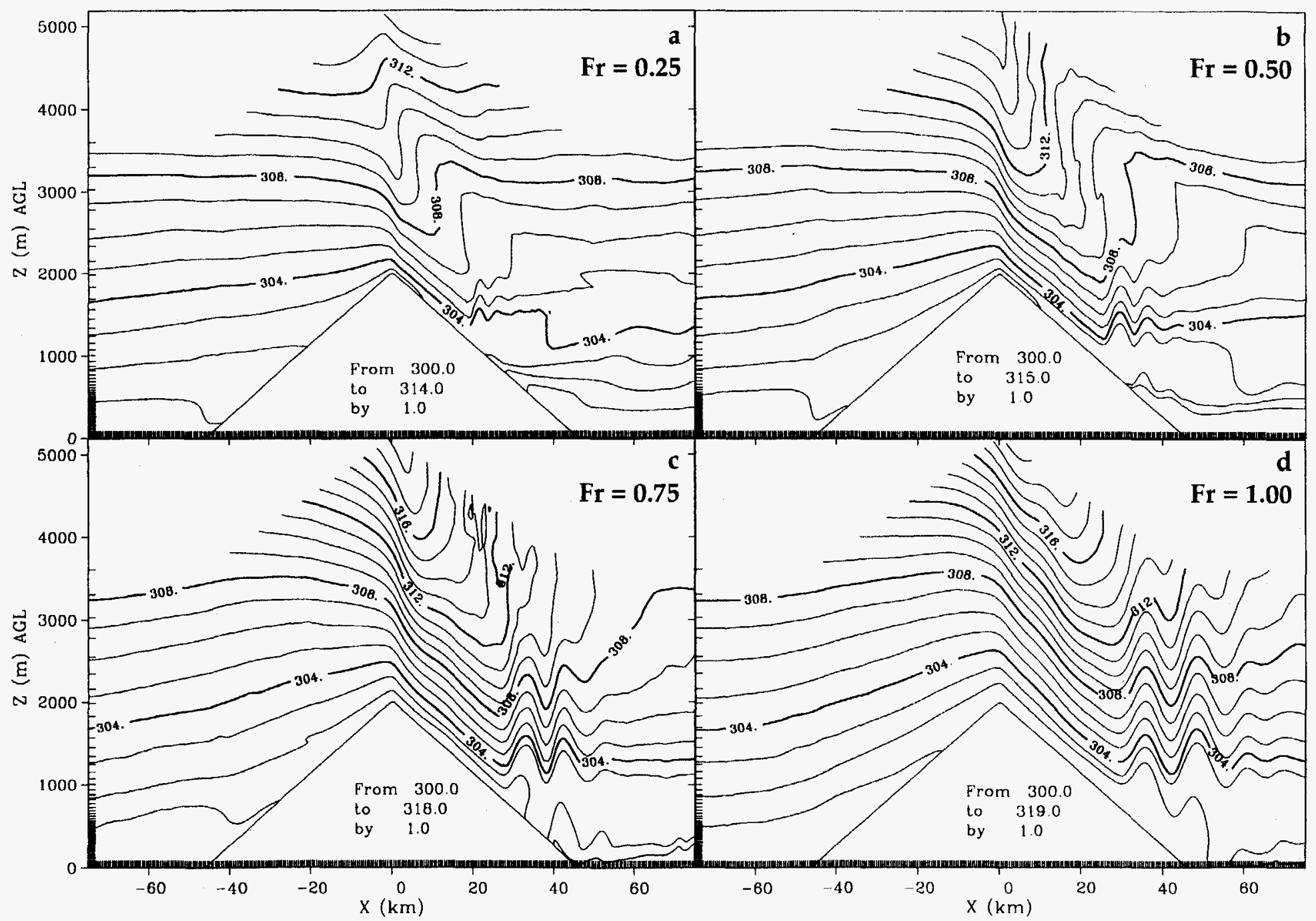

Figure 5.5 Potential temperature contours at 3 hours into the, a) $\mathrm{Fr}=0.25, \mathrm{~b}$ ) $\mathrm{Fr}=0.50, \mathrm{c}) \mathrm{Fr}=0.75$ and d) $\mathrm{Fr}=1.0, \mathrm{MWO} 2$ dimensional simulations. 
each simulation in Figure 5.5. The vertical potential temperature contours in panels $a-c$ verify that wave breaking occurs downstream of the barrier for $\mathrm{Fr}=0.25$ and 0.50 and at higher levels for $\mathrm{Fr}=0.75$. For $\mathrm{Fr}=1.0$ potential temperature contours are nearly vertical in places, but never become completely unstable. Wave structure, as in Figure 5.5, is seen to descend a smaller distance downslope as Fr decreases. From this behaviour it is logical to conclude that higher Fr flow will be more likely to scour katabatic flow than lower $\mathrm{Fr}$ flow and that high Fr flow will scour a greater distance downslope relative to low Fr flow. Additionally, for lower Fr, weak flow will exist on both the up and downstream sides of the barrier at low levels, where katabatic flow is more likely to form. The strong pressure perturbations within the topographically anchored portion of the wave and those lee waves downstream, are also likely to impact the surface flow variability. Since the evolution of these waves is non-steady, their evolution is expected to inherently effect those flows, such as katabatic flows, it interacts with.

\subsubsection{Katabatic flow with mountain wave}

To investigate the interaction of mountain waves with katabatic flow, the same simulations as completed in the previous subsection were repeated but with the radiation parameterization active. Katabatic flow was allowed to develop completely ( 6 hours) before ambient flow was linearly increased over 30 minutes to the wind speed appropriate to the Fr of interest. This wind 'spin-up' occurred at altitudes above $500 \mathrm{~m}$ above the lowest topography. It was therefore ensured that katabatic flow was well-developed prior to the existence of the mountain wave forcing, and their interaction would be obvious. This spinup configuration also was the reason for the use of $\mathrm{H}=1500 \mathrm{~m}$ in the $\mathrm{Fr}$ calculations.

Figures 5.6 -5.9 show u component winds and potential temperature for each of the four 


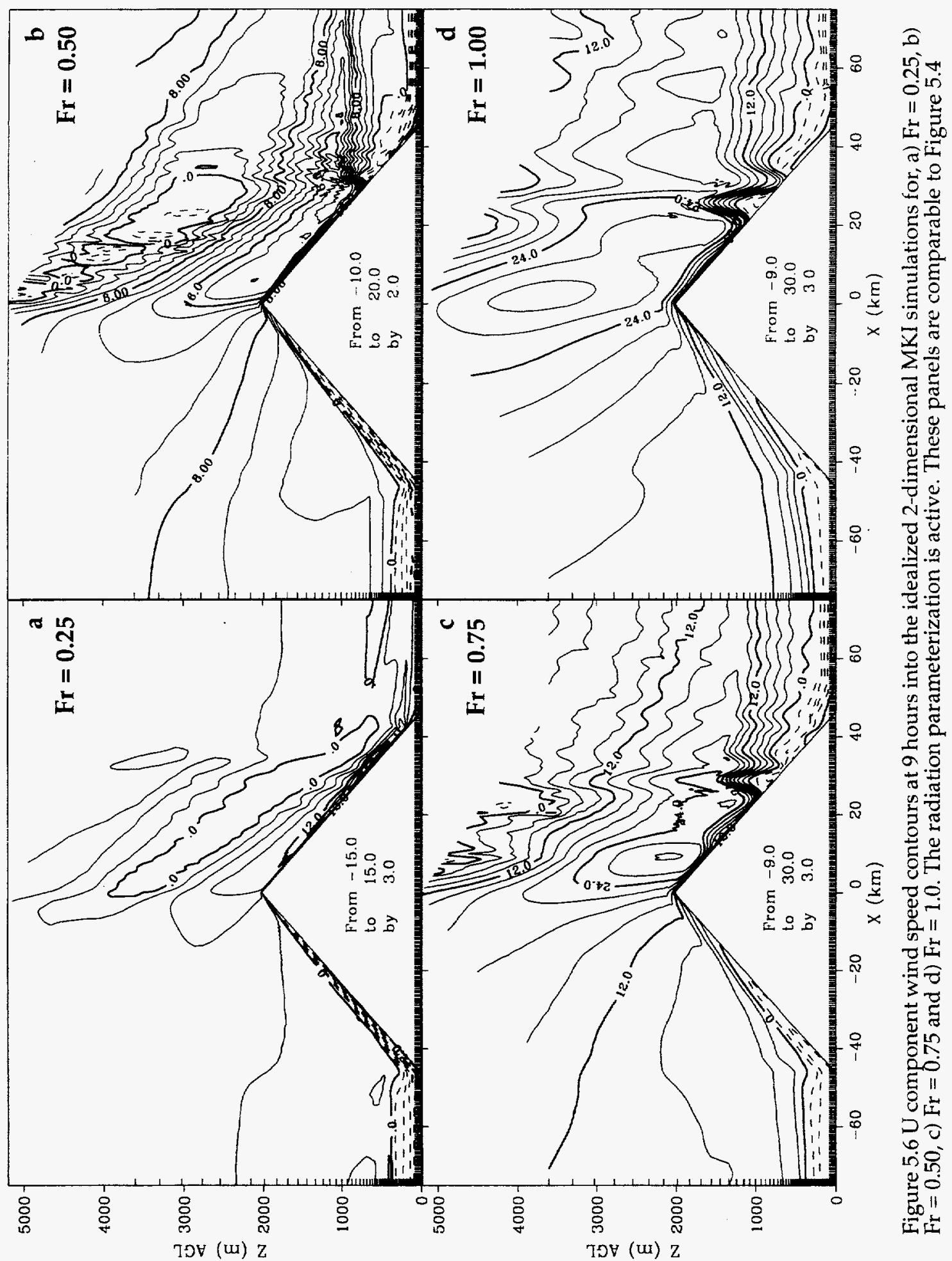




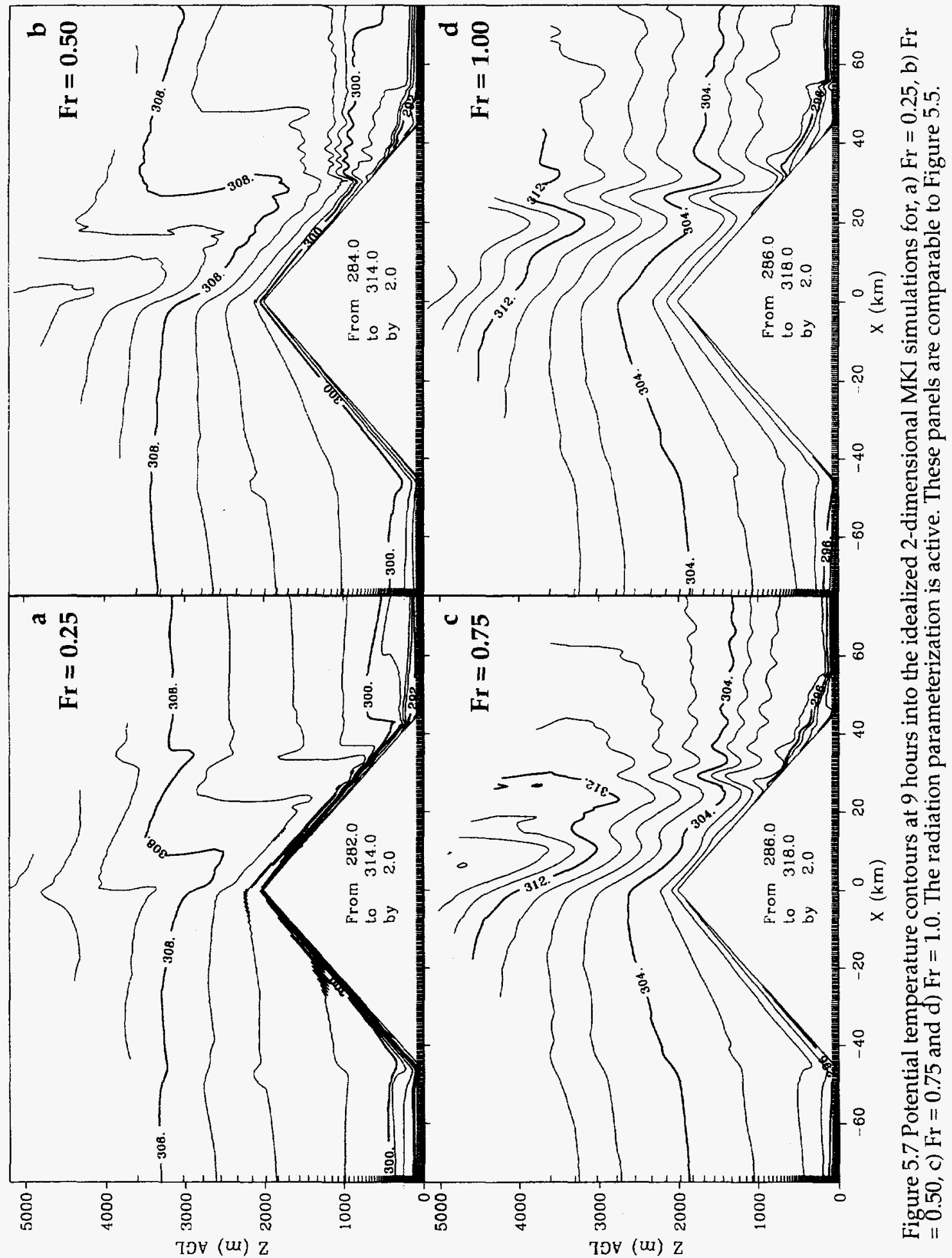




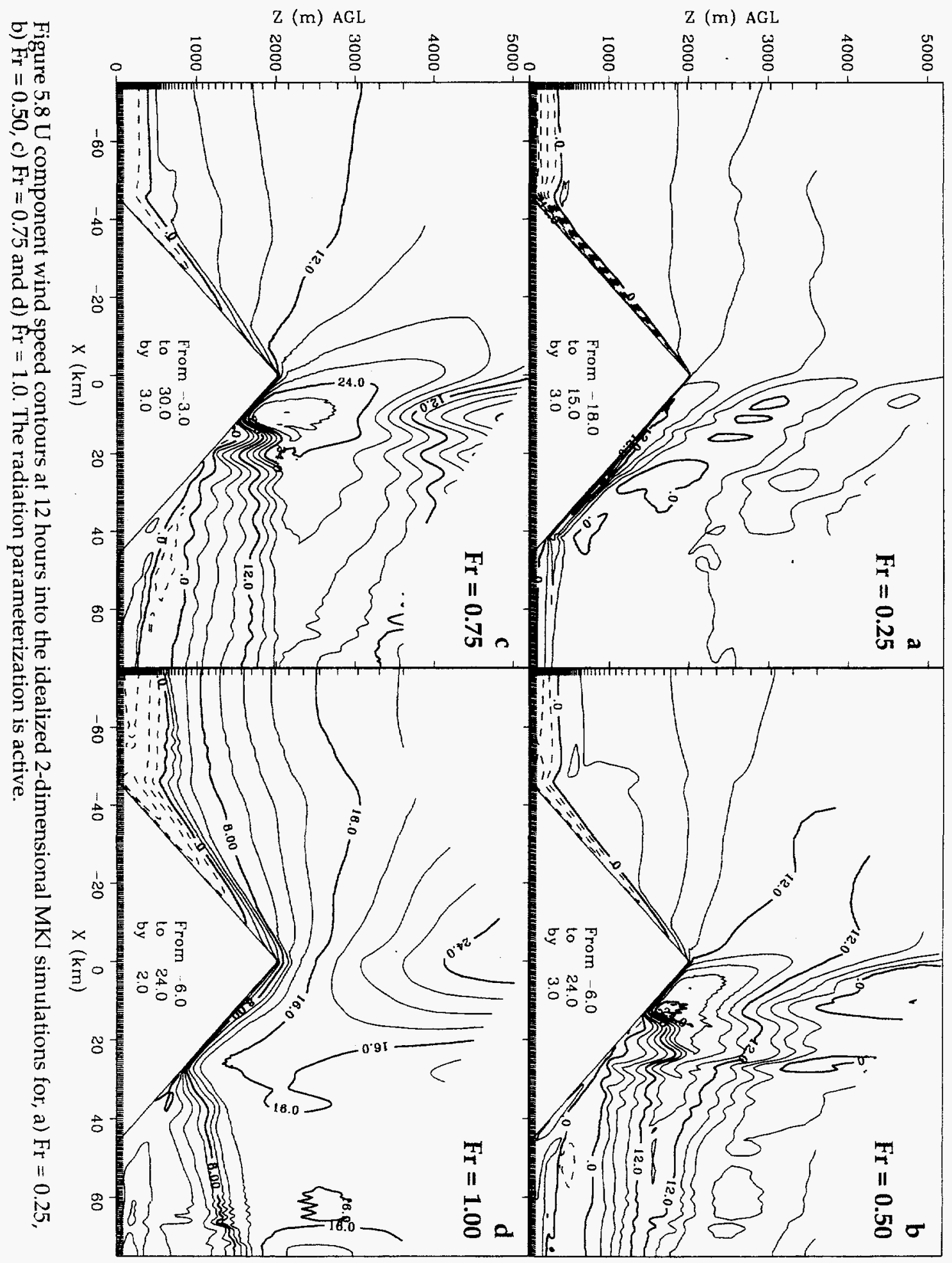




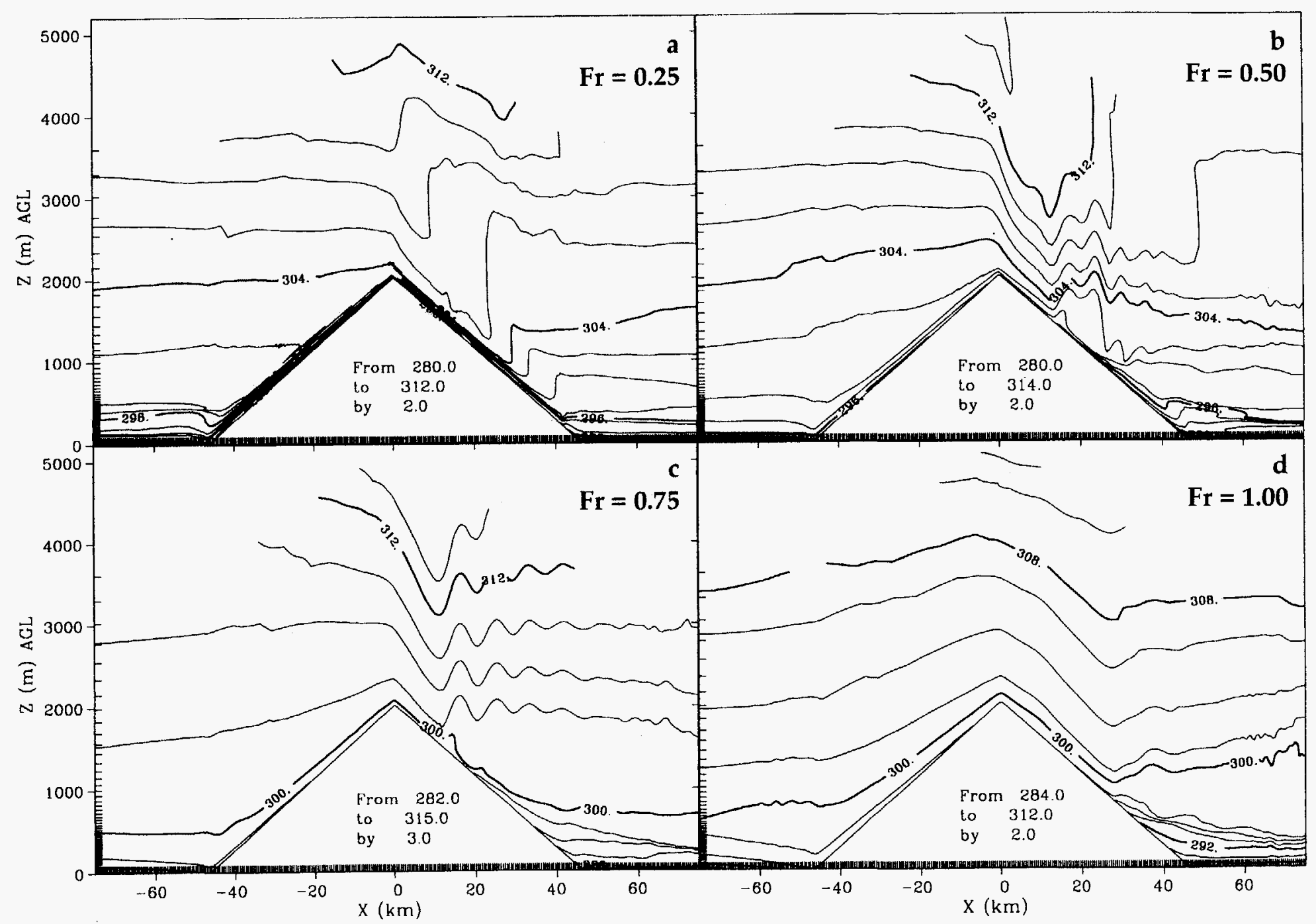

Figure 5.9 Potential temperature contours at 12 hours into the idealized 2-dimensional MKI simulations for, a) $\mathrm{Fr}=0.25, \mathrm{~b}$ ) $\mathrm{Fr}$ $=0.50, \mathrm{c}) \mathrm{Fr}=0.75$ and $\mathrm{d}$ ) $\mathrm{Fr}=1.0$. The radiation parameterization is active. 
simulated Froude numbers $(0.25,0.50,0.75$ and 1.00$)$ at 9 hours and 12 hours into the simulation. However, since winds were spun up to the respective Fr at 6 hours into the simulation, 9 hours in these MKI simulations is equivalent to 3 hours with regard to mountain wave evolution in the mountain wave only simulations described in the previous subsection. Therefore, Figures 5.6 and 5.7 are comparable to Figures 5.4 and 5.5, respectively. The reader should note that symmetric katabatic flows existed on the mountainside prior to the onset of mountain wave forcing. These flows increased in depth with distance downslope, and were like those in the undisturbed katabatic flow, idealized case of Section 5.2.1 and Figure 5.1, until the 6 hour point.

At 9 hours into the simulation (or 3 hours from the onset of mountain wave forcing), mountain wave structure is evident in all panels of Figures 5.6 and 5.7. At each $\mathrm{Fr}$ from $0.25-1.0$ there are significant differences between the wave under the influence of radiative cooling and that without (Figures 5.4 and 5.5). Generally, katabatic flow is dramatically influenced by any of the mountain waves, least so by $\mathrm{Fr}=0.25$ (panel $a$ ). But stratification on the west side of the barrier, combined with a favorable pressure gradient, protects katabatic flow from complete erosion there. In fact, easterly u winds are stronger in all 4 cases compared to the mountain wave only simulations. On the whole it is difficult to proportion how much of this flow is forced by mountain wave dynamics and how much is forced by katabatic-type dynamics, but it is certainly some combination as found by Arritt and Pielke (1986) in a modeling study and in the observations of Mursch-Radlgruber (1995). The stronger near surface thermal stratification on the western slopes of all panels in Figure 5.7, weighs considerably in favor of katabatic forcing being the primary driver. Furthermore, a comparison of the flow for, say, $\mathrm{Fr}=0.50$, shows that without radi- 
ative cooling the blocked flow measures $\sim 2 \mathrm{~m} \mathrm{~s}^{-1}$ (see Figure 5.4b), but with this effect the return flow is as high as $\sim 10 \mathrm{~m} \mathrm{~s}^{-1}$. By the same token, with only radiative forcing the western side flow reaches $13.5 \mathrm{~m} \mathrm{~s}^{-1}$ for the given cooling on Figure $5.7 \mathrm{~b}$, but with mountain wave forcing the katabatic flow only reaches $\sim 10 \mathrm{~m} \mathrm{~s}^{-1}$. Similar statements can be made for each of the other Froude number flows. Generally the existence of mountain wave flow on the western side reduces the strength of the katabatic flow than would otherwise exist, and this reduction is greater as the strength of flow increases (or Fr increases). This occurs because a weaker pressure gradient force exists due to the lessened cooling at the surface.

On the eastern side of the barrier at 9 hours large changes in wave, and especially katabatic flow, behaviour can be seen in Figures 5.6 and 5.7. For $\mathrm{Fr}=0.25$ the mountain wave adheres to the mountain side for a greater downslope distance than without radiative cooling active (compare panel $a$ of Figures 5.6 and 5.7 to panel $a$ of Figures 5.4 and 5.5. This effect, which has been noted by Scorer (1967), also occurs for $\mathrm{Fr}=0.50$, but not for $\mathrm{Fr}=0.75$ or 1.0 . For a given stability and barrier height it appears that lower wind speed mountain waves are 'captured' by the additional thermal stability caused by radiative cooling, but at higher wind speeds the mountain wave detaches from the slope somewhat sooner than without radiative cooling. The latter effect is more intuitively understood than the former, because one would expect a lowering of Froude number with increasing $\mathrm{N}$ due to radiative cooling. Returning to our analysis of the $\mathrm{Fr}=0.25$ case, we see from Figure 5.6a that the downward phase of the mountain wave on the eastern side of the mountain has essentially combined with the katabatic flow there. Flow speed along the mountainside is actually stronger than in the mountain wave only case or the katabatic flow case, 
showing that the momentum of the wave has augmented that of the katabatic flow and the two phenomena seem indistinguishable. This is verified by the continuity of stratification on the eastern side seen in Figure 5.7a. Figure 5:7a also shows that vertical isentropes exist very near the lowest topography, whereas in Figure 5.5a wave breaking only occurred approximately halfway down the mountainside. Clearly, though both mountain wave and katabatic flow exist in these simulations, their behaviour and evolution is drastically changed from what it was as independent entities. In fact, the term 'katabatic flow' no longer seems appropriate terminology to describe the flows on the eastern side, because katabatic-type forcing is only a portion of the dynamical reason for this flow.

For higher Fr than 0.25 , a return flow toward the barrier occurs on the eastern side of the barrier at low levels. In each case, however, this return flow lies above a very shallow layer of weak $\left(<3.0 \mathrm{~m} \mathrm{~s}^{-1}\right)$ katabatic flow. Thus, strong near surface thermal stability, has allowed the development of katabatic flows at low elevations despite the destructive influence of mountain wave momentum. In contrast, along the upper slopes katabatic flow has been completely scoured, and flow there is dominated by mountain wave momentum. As expected, thermal stability in these upper altitudes, is increasingly less as the strength of the scouring momentum or size of $\mathrm{Fr}$, increases (see near surface potential temperature on Figure $5.7 \mathrm{a}-\mathrm{c})$. It is intriguing to note that for $\mathrm{Fr}=0.50$ the scouring of katabatic flow occurs further downslope than for its higher Fr brethren, at least for this time. At altitudes less than the wave separation point, weaker flow and stronger thermal stability develop. The number of strong downstream lee waves in the $\mathrm{Fr}=0.75$ and $\mathrm{Fr}=1.00$ cases has also been reduced by the inclusion of radiative cooling.

At 12 hours, or 6 hours after the beginning of full mountain wave forcing, the flow has 
evolved to a considerably different form, as shown in Figures 5.8 and 5.9. For Fr $=0.25$ (panel $a$ in either Figure), continued radiative cooling has driven stronger katabatic flows on the western side than at 9 hours, but those on the eastern side are approximately the same. The mountain wave structure is still embedded within the stable layers that also generate the katabatic flow. Waves continue to break on the eastern side, but, due to increased stratification, not as close to the lowest topography. Stronger stratification has brought the separation point of the $F r=0.5$ and 0.75 waves upstream significantly from their 9 hour position, though the wave for $\mathrm{Fr}=0.5$ still separates at a lower altitude than for $\mathrm{Fr}=0.75$. Accordingly, deeper, stronger katabatic flow develops in the deeper, relatively quiescent zone underlying this separation point for $\mathrm{Fr}=0.75$. Return flow remains elevated above the katabatic flow, but is weaker and less continuous than at 9 hours. For Fr $=1.0$ in panel $d$ of either Figure, lee waves have completely disappeared from the mountain wave structure but the separation point remains nearly halfway down the mountain. Low level stable layers on the eastern side of the barrier remain strong with a nearly neutral layer above, though return flow has disappeared. On the western side, katabatic flow twice as strong as that at 9 hours has developed and has penetrated to higher altitudes near mountaintop.

\subsection{THREE-DIMENSIONAL IDEALIZED SIMULATIONS}

In addition to a 3-d katabatic flow only simulation, simulations with mountain wave forcing were also completed, but only for the $\mathrm{Fr}=0.5$ case. It can be argued that 2 -dimensional simulations are insufficient to describe the complicated interaction of mountain waves with katabatic flow. This argument states that in 2-d, turbulence can transfer energy upscale, whereas in 3-dimensions upscale transfer is typically small, with the overall 
effect being downscale energy transfer (e.g. Kraichnan 1976). Since, even in our 2-d simulations, turbulent dissipation is primarily introduced via a parameterization - a parameterization that regardless of dimension removes energy - we expect that they will perform reasonably well. For completeness, 3-d simulations are briefly described here. We have already seen in Section 5.2.1 that the 3-d katabatic flow only simulation is very similar to the 2-d katabatic flow only simulation, but katabatic flow is typically less turbulent than mountain wave flow. Thus, we may expect more significant differences between 2- and 3d simulations, as mountain wave forcing, with a greater tendency to create turbulence, is added. The configuration of the 3 dimensional simulations is exactly like its $2-\mathrm{d}, \mathrm{Fr}=0.50$ counterpart, except that 21 grid points have been added in the north-south direction with cyclic boundary conditions $(\Delta y=500 \mathrm{~m})$.

The results of these simulations were very similar to their two-dimensional counterparts. This similarity was described in Section 5.2.1 for the katabatic flow only simulation. Proof of the similarity for the MWO and MKI simulations is found in Figure 5.10, where panels $a-d$ are comparable to panel $b$ of Figures $5.4-5.7$, respectively. The reader can see that, indeed, these model results compare very closely to the 2-dimensional simulations, even in minute details. Temporally the similarities remain, so that the descriptions of the $2-d$ simulations in the prior two sections, also adequately describe the interaction of mountain waves and katabatic flow in 3-d.

\subsection{SUMMARY}

From the above described idealized simulations we have found that mountain waves and katabatic flow interact in a complicated, non-linear, fashion. Specifically, as the mountain wave structure evolves in response to changing stratification, the katabatic flow 


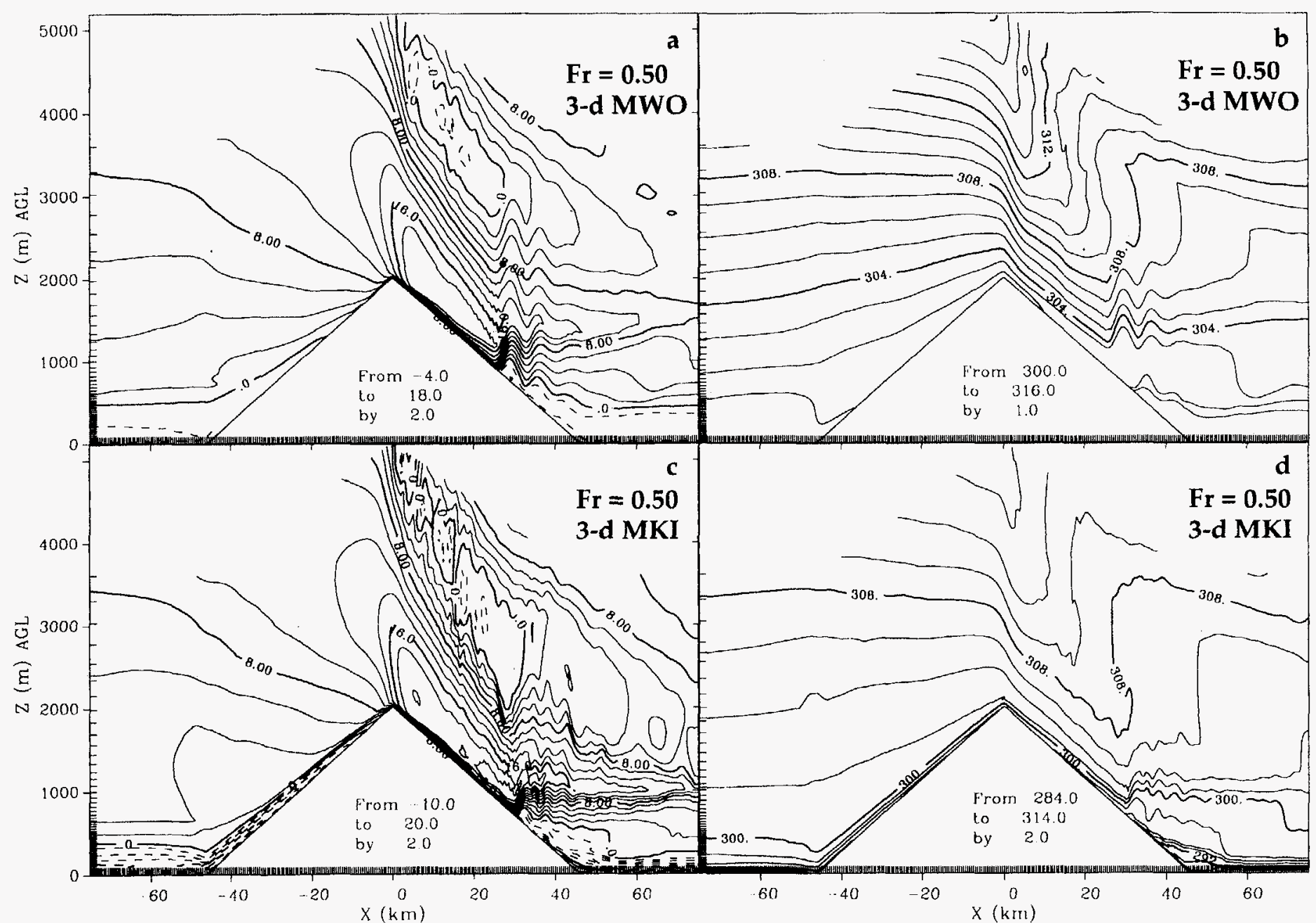

Figure 5.10 East-west vertical cross sections through the center of the 3-dimensional idealized, $\mathrm{Fr}=0.50$, mountain wave only simulations. a) $\mathrm{u}$ component winds for the mountain wave only simulation at 3 hours comparable to Figure $5.4 \mathrm{~b}$, b) Potential temperature contours for the mountain wave only simulation at 3 hours comparable to Figure $5.5 b, c) u$ component winds for the MKI simulation at 9 hours comparable to Figure 5.6b, and d) Potential temperature contours for the MKI simulation at 9 hours comparable to Figure $5.7 \mathrm{~b}$. 
underneath it must also evolve. Strong nocturnal stratification is found to be able to create katabatic forcing quite easily on the upstream side of the barrier (as enhanced by the near surface, dynamic pressure increase as a consequence of blocking), though generally weaker than would exist without wave forcing due to better mixing of cold air. This mechanism may have been measured by Mursch-Radlgruber (1995). On the downstream side of the barrier, upstream of the mountain wave separation point, katabatic flow is completely scoured for high $\mathrm{Fr}$ (high wind speed). For smaller Fr, such as 0.25 , the downward mountain wave phase is found to couple with the katabatic flow. and even enhance its speed. Also, at $\mathrm{Fr}<\sim 0.75$, the mountain wave under the influence of radiative cooling, is found to adhere to the topography for a larger distance downslope, as suggested may happen by Scorer (1967), but for $\mathrm{Fr}>\sim 0.75$, the mountain wave adheres to topography less than it would if radiative cooling were not occurring. The latter effect is logical as increasing stratification will in turn increase $\mathrm{N}$, which will decrease $\mathrm{Fr}$, such that the separation point should move uphill. Overall, a highly variable, evolutionary state is found where mountain waves and katabatic flow interact. Since the Froude number climatology in Chapter 4 showed that Fr is generally between 0.2 and 0.7 , the likelihood of mountain wave katabatic flow interaction along the Front Range is high and should be significant. 


\section{CHAPTER 6}

\section{REAListic TOPOGRAPHY MOdEL RESUltS}

\subsection{OVERVIEW}

The idealized model results of the previous chapter give us significant insight into how mountain wave - katabatic flow interaction might manifest itself under ideal conditions. To take our understanding of MKI to a higher level however, requires analysis of a realistic situation. This chapter describes the results of realistic topography 3-d modeling of a case night from the ASCOT 1993 experiment where MKI was likely. Of the possible nights during the experiment, examples of which were described in Chapter 3, the night of 03-04 Sep 1993 was selected (see Section 3.3.3).

\subsection{MODEL SET-UP}

The following series of steps were followed to set-up the RAMS configuration in an appropriate way:

1. Determine relevant scales.

2. Determine required model grid spacing to capture relevant scales.

3. Determine sufficient horizontal and vertical coverage for phenomena and study area.

4. Determine locations and time periods of ASCOT 1993 data sites that will used for comparison with model results.

5. Reconfigure grids such that a considerable number of observational sites are easily 
compared with model output.

6. Select appropriate grid points for high time resolution output of model data (every 2.0 minutes).

7. Of the possible MKI case nights from the ASCOT observations determine which ones have fewest data gaps and sufficient MAPS initialization data available.

8. Select case night of most interest.

9. Set the simulation length so as to encompass night of interest, including time for model adjustment and considering differential slope heating effects.

The scale of the katabatic flow, mountain wave and topography were determined to define the relevant scales to the problem. The topographic scale relevant to the katabatic flow for the Front Range is considered to be the width of the canyon of interest and the distance from the Continental Divide to the plains $(\sim 45 \mathrm{~km})$. Since numerical modeling is most efficiently completed over larger scales, the largest canyon whose atmosphere was observed during the ASCOT 1993 field program, Eldorado Canyon, was chosen as the topographic focus of the simulations. Eldorado Canyon is clearly larger in length $(\sim 40$ $\mathrm{km}$ ), average ridge-to-ridge width $(\sim 8 \mathrm{~km})$ and depth (up to $700 \mathrm{~m})$ than Coal Creek Canyon (see Figure 3.1). The scale of the katabatic flow can thus be considered to have approximately the same dimensions. The mountain wave scales are much larger, however. Mountain waves can propagate into the stratosphere, which requires a domain depth of over $10 \mathrm{~km}$. The vertical wavelength of mountain waves depends on conditions but typically ranges from $4-10 \mathrm{~km}$. The horizontal scale of mountain waves for a real case study is considerably larger than for idealized cases where the upstream flow can be specified. For real cases the distance a synoptic disturbance would travel during the overnight period ( 12 hours) is minimally required on either side of the domain. From Figure 3.2 we note 
that the $500 \mathrm{mb}$ wind near the study area on the case night 3 - 4 Sep 1993 was no larger than $15 \mathrm{~m} \mathrm{~s}^{-1}$. If we assume the $500 \mathrm{mb}$ wind speed represents the typical progress of a synoptic disturbance, we find that it would travel $650 \mathrm{~km}$ in 12 hours (12 hours is the simulation length). This, in turn, requires at least a $1300 \mathrm{~km}$ domain centered over the study area. The mountain wave phenomena also requires that the barrier over which it resides be adequately resolved. For this value, the relevant scale is the distance from the Continental Divide to the plains, or $\sim 45 \mathrm{~km}$.

Using the above scales, and the fact that topographic features and atmospheric phenomena are well resolved when defined by $10 \Delta x$, the required grid spacing for a good simulation of all the scales relevant to MKI in the Front Range can be determined. These requirements are summarized in Table 6.1. From the Table it is evident that a grid configuTable 6.1: Relevant scales, required grid spacing and actual grid spacing for the MKI phenomena in the Front Range of the Rockies.

\begin{tabular}{|c|c|c|c|c|}
\hline Feature of relevant scale & Scale & $\begin{array}{c}\text { Required } \\
\text { Grid Spacing }\end{array}$ & $\begin{array}{c}\text { Grid Spacing } \\
\text { Used }\end{array}$ & Comments \\
\hline & $(\mathrm{m}$ or $\mathrm{km})$ & $(\mathrm{m}$ or $\mathrm{km})$ & (m or $\mathrm{km})$ & \\
\hline \multicolumn{5}{|l|}{ Katabatic Flow: } \\
\hline Horizontal (Eldorado Canyon) & $8000 \mathrm{~m}$ & $800 \mathrm{~m}$ & $400 \mathrm{~m}$ & On Grid 5 only \\
\hline Vertical (flow depth) & $500 \mathrm{~m}$ & $50 \mathrm{~m}$ & $20 \mathrm{~m}$ & On Grid 5 only \\
\hline \multicolumn{5}{|l|}{ Mountain Wave: } \\
\hline Horizontal (Synoptic movement) & $1300 \mathrm{~km}$ & $130 \mathrm{~km}$ & $57.6 \mathrm{~km}$ & On Grid 1 only \\
\hline Vertical (Stratosphere) & $12000 \mathrm{~m}$ & $1200 \mathrm{~m}$ & $400 \mathrm{~m} \mathrm{Max}$ & At high altitudes \\
\hline Vertical Wavelength & $4000 \mathrm{~m}$ & $400 \mathrm{~m}$ & $400 \mathrm{~m} \mathrm{Max}$ & At high altitudes \\
\hline
\end{tabular}

ration sufficient to capture the phenomena of interest will require grid nesting - it is not feasible to cover the synoptic scale domain with the grid spacing minimally required by katabatic flow scales $(\Delta x=800 \mathrm{~m}, \Delta \mathrm{z}=50 \mathrm{~m})$ For these simulations, covering the entire synoptic domain without grid nesting would require a grid configuration of $\sim 1500 \times 1500$ 
$\times 80$ grid points for a total of $180 \times 10^{6}$ nodes!

To this end a RAMS model configuration was devised with the attributes shown in

Table 6.2. These parameters were chosen to be as realistic as possible to the case night of interest. Boundary conditions were chosen based on a series of sensitivity tests with mountain wave and katabatic flow simultaneously occurring (see also Chapter 4). It was found that the Klemp-Wilhelmson lateral boundary condition and the impervious lid with a number of Rayleigh friction absorbing layers for the upper boundary condition worked Table 6.2: Model configuration details for case study simulations (additional details in text).

\begin{tabular}{|l|l|}
\hline \multicolumn{1}{|c|}{ Characteristic } & \multicolumn{1}{|c|}{ Configuration } \\
\hline \hline Number of grids & 5 \\
\hline Simulation length & 12 hours \\
\hline Vertical grid spacing style & $\Delta z$ constant to $720 \mathrm{~m} \mathrm{AGL,} \mathrm{then} \mathrm{stretched} \mathrm{to} \Delta \mathrm{z}=400 \mathrm{~m}$ \\
\hline Domain focus & Rocky Mountain barrier and Eldorado Canyon \\
\hline Output & Meteorological variables and model tendencies \\
\hline Initial time and date & $00 Z, 4$ Sep 1993 (0500 LST, 3 Sep 1993)) \\
\hline Initialization & Horizontally homogeneous or with MAPS data \\
\hline Terrain data resolution & 30 s ( 800 m) on Grids 1-3, 3s ( 80 m) on Grids 4 and 5 \\
\hline Equation set & Non-hydrostatic, compressible \\
\hline Turbulent diffusion & Vertical - Level 2.5, Horizontal - K theory \\
\hline Lateral boundaries & Klemp-Wilhelmson (1978) radiative or nudged (MAPS) \\
\hline Vertical boundaries & Top - Lid with 10 absorbing layers, Bottom - No slip \\
\hline Roughness length & As derived from vegetation data set \\
\hline Filters & None \\
\hline Radiation & Short- and longwave parameterization (updates per 600 s) \\
\hline Soil (parameterized) & 11 layers, Silty clay loam, 30\% of saturation \\
\hline Vegetation (parameterized) & From lat/lon data set \\
\hline
\end{tabular}

best together for the phenomena of interest subject to horizontally homogeneous initialization. For the realistic simulation, with four dimensional data assimilation (4DDA) using MAPS data (see Section 3.2.5), the Davies technique was used. This technique provides 
boundary conditions through dynamic relaxation (Davies 1976, Davies and Turner 1977). A generic soil type of silty clay loam at $30 \%$ saturation was used for lack of a gridded data set containing this information (see McCumber and Pielke 1981, and Tremback and Kessler 1985, for details on the USDA soil types and soil parameterization). Vegetation, however, was reasonably well represented due to the existence of a lat-lon data set of vegetation type at 30s spatial grid increment as described by Eidenshink (1992). A radiative parameterization devised by Mahrer and Pielke (1977) for both long- and shortwave radiation was used. This parameterization is unable to account for cloud radiative effects. This is not a serious limitation, however, because clouds are not a significant factor during this case night, nor are clouds able to develop in this model configuration (no cloud microphysics).

The simulation length was chosen to be 12 hours, beginning near sunset before the case night $(00 \mathrm{Z}$ or $01 \mathrm{Z})$. After repeated tests the author has found that to adequately simulate katabatic flows there are two possible configurations, 1) where the simulation begins at approximate sunrise the day prior and runs for 24 hours or, 2) where the simulation begins at sunset and runs for 12 hours. At either of these two start times the ground and near-surface air temperature are nearly equal and an atmospheric stability change (under clear skies, anyway) is occurring. Simulations that begin midday often contain serious inconsistencies, particularly in complex terrain, because heating by insolation is favored on western and southwestern slopes during afternoon. Without a full day of insolation unrealistic east-west temperature gradients and wind fields develop that contaminate the overnight katabatic flow development. Further, a mid-day simulation start does not allow sufficient CBL development so that katabatic flow descends into an unrealistic atmospheric stratif- 


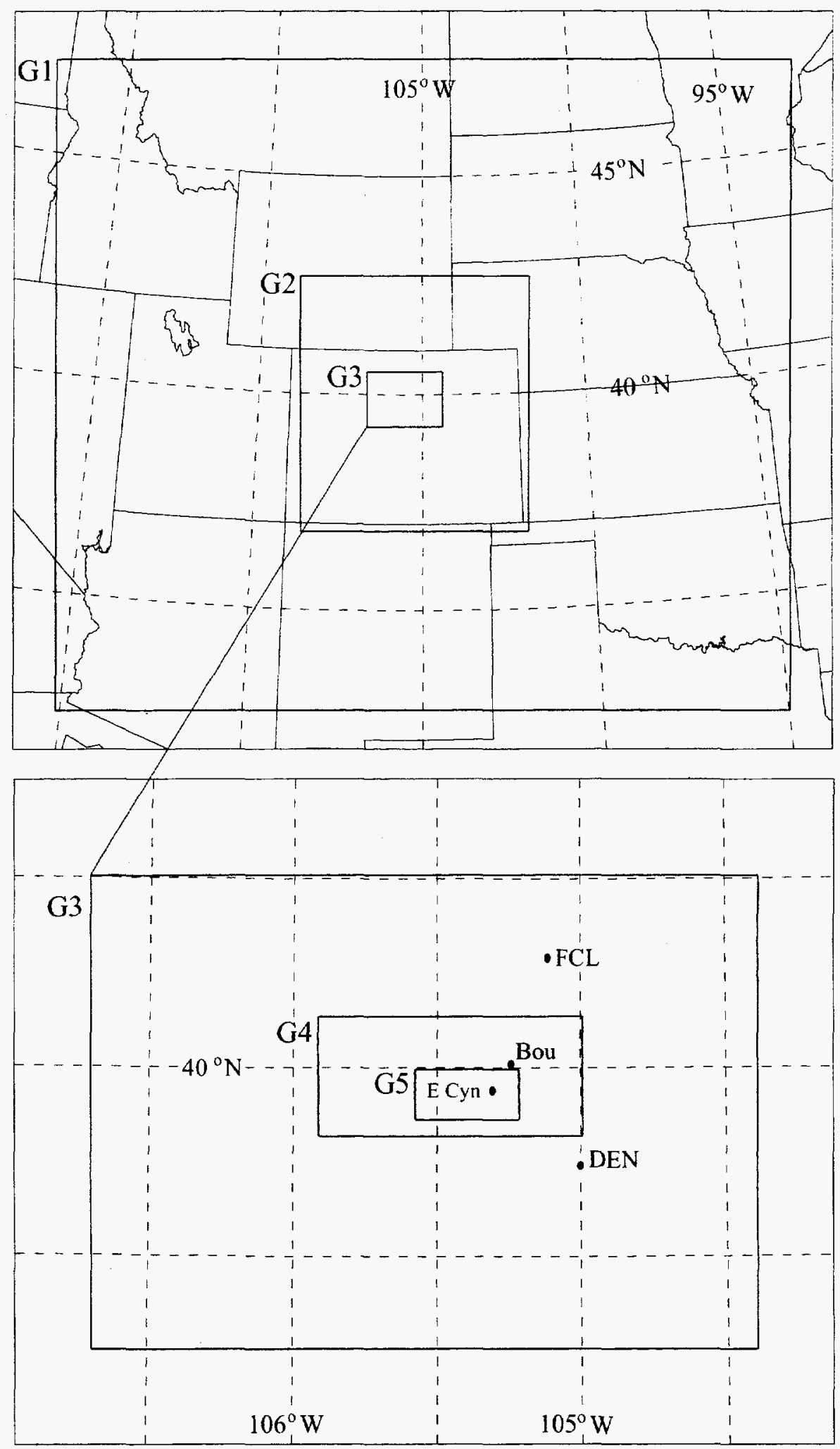

Figure 6.1 The 5 grids used in the realistic simulations. Grid 3 is shown in both the upper and lower portions of the figure for reference purposes. Note that the grids sequentially telescope down to the region of interest on Grid 5, which overlies Eldorado Canyon. 


\section{Gregory S. Poulos}

cation. Even in the case where 4DDA is used, oftentimes initialization and subsequent files do not contain sufficient information on the existing CBL to allow a mid-day initialization. For these simulations a start time near sunset was sufficient, although for the first few hours of simulated time the model is partially adjusting to the initial conditions.

Table 6.3 details the grid spacing and node distribution used. The domain sizes for each grid were selected to sufficiently resolve the required scales. Noting Figures 6.1 and 6.2a, it is clear that the Grid 1 domain is sufficiently large to capture synoptic scale motions as communicated on its outermost grid points from the MAPS data sets. Grids 2 and 3 (Figure $6.2 \mathrm{~b}$ and $\mathrm{c}$ ) encompass large scale terrain features that may have an influence on mountain wave evolution; the entire State of Colorado and the Northcentral Colorado Rockies, respectively. Grid 4 is rectangular and crosses the Continental Divide with a 1.6 $\mathrm{km}$ grid interval while also extending well onto the plains around Rocky Flats (Figure

Table 6.3: Model information by grid for case study simulations

\begin{tabular}{|c|c|c|c|c|c|c|}
\hline Grid & $\Delta \mathrm{x}, \Delta \mathrm{y}$ & $\Delta z_{\min }$ & $\mathrm{nx} \times \mathrm{ny} \times \mathrm{nz}$ & \#Nodes & Domain Size $\times \mathrm{y} \mathrm{z}(\mathrm{km})$ & $\Delta \mathrm{t}(\mathrm{s})$ \\
\hline \hline 1 & $57.6 \mathrm{~km}$ & $60 \mathrm{~m}$ & $34 \times 30 \times 65$ & 66,300 & $1900 \times 1670 \times 17$ & 60.0 \\
\hline 2 & $19.2 \mathrm{~km}$ & $60 \mathrm{~m}$ & $32 \times 35 \times 65$ & 72,800 & $595 \times 653 \times 17$ & 30.0 \\
\hline 3 & $4.8 \mathrm{~km}$ & $60 \mathrm{~m}$ & $42 \times 30 \times 65$ & 81,900 & $197 \times 139 \times 17$ & 10.0 \\
\hline 4 & $1600 \mathrm{~m}$ & $60 \mathrm{~m}$ & $50 \times 23 \times 65$ & 74,750 & $78 \times 35 \times 17$ & 3.3 \\
\hline 5 & $400 \mathrm{~m}$ & $20 \mathrm{~m}$ & $78 \times 38 \times 86$ & 254,904 & $31 \times 15 \times 17$ & 1.1 \\
\hline Total & & & & 550,654 & & \\
\hline
\end{tabular}

6.2d). Grid 4 is intended to capture mountain wave structure with great detail as air passes over the Continental divide. Within Grid 4, Grid 5, shown in Figure 6.2e, encompasses the majority of Eldorado Canyon's drainage area with $400 \mathrm{~m}$ horizontal grid spacing, but does not cross the Continental Divide. Grid 5 utilizes vertical grid nesting in its lowest layers allowing vertical grid spacing of $\sim 20 \mathrm{~m}$ for $500 \mathrm{~m} \mathrm{AGL}$; the same vertical grid 


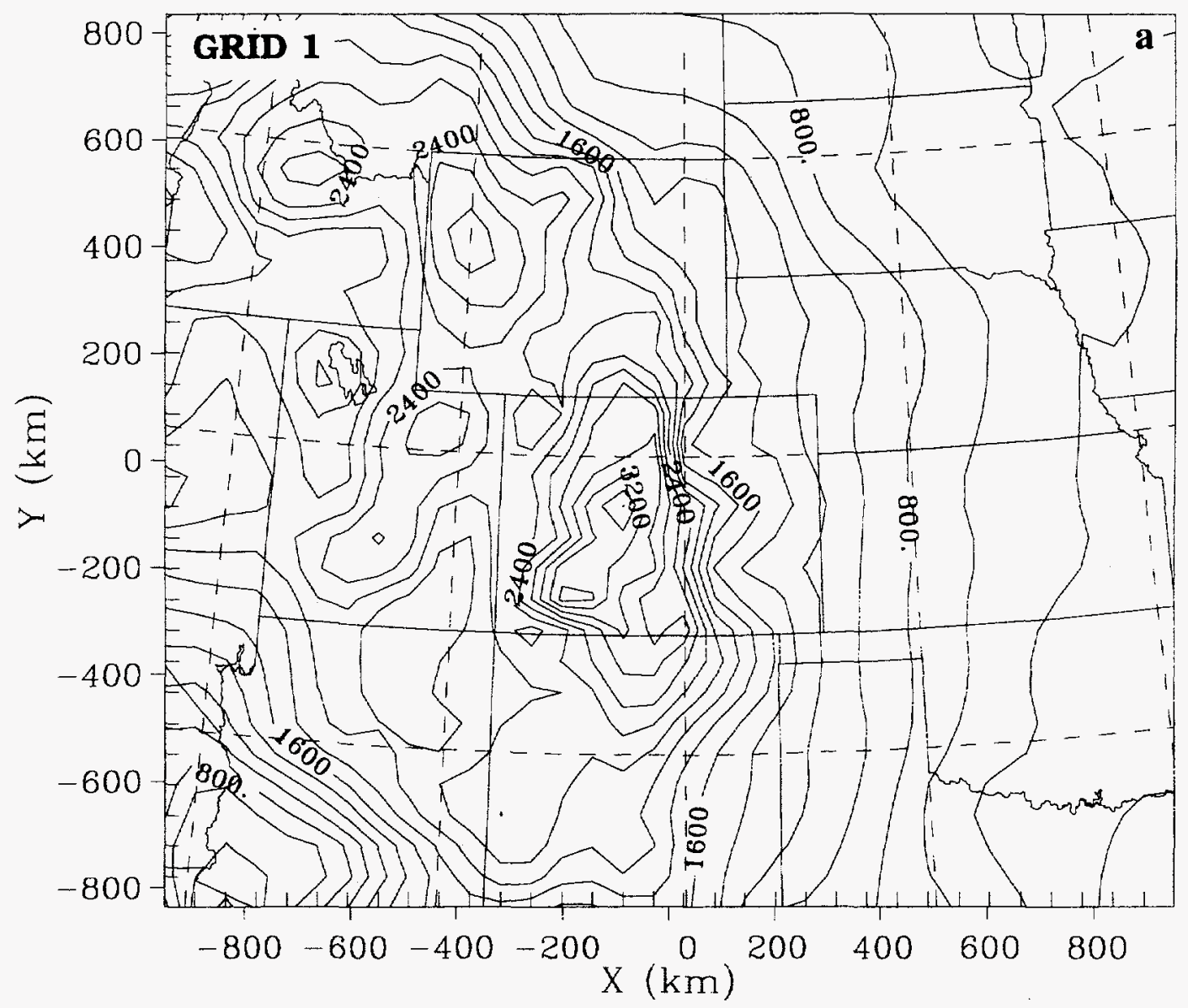

Figure 6.2 RAMS topographical representations for each of the 5 grids (1-5 on panels $a$-e, respectively) used for realistic simulations; a) Grid 1, whose southwest corner is at $32.00^{\circ} \mathrm{N}$, $115.39^{\circ} \mathrm{W}$, lat-lon increments in dashed lines every $5.0^{\circ}$, topographic contours every $200 \mathrm{~m}$. spacing as in the idealized simulations (see Chapter 5). Considerable care was taken to ensure that vertical stretch ratios were close enough to 1.0 to retain accuracy in vertical differencing. Using vertical grid stretching, all grids reach to $17 \mathrm{~km} \mathrm{MSL}$.

With this configuration, for each long time step the smallest scale grid with the most grid points, Grid 5, had to execute 54 times. Correspondingly, these simulations ran slower than real time; about 6 days to completion on a dedicated IBM 590 RISC/6000, or approximately 12 times slower than real time.

\subsection{SENSITIVITY STUdy FraMEWORK}

Since our interest is the interaction of two non-linear features, mountain waves and 


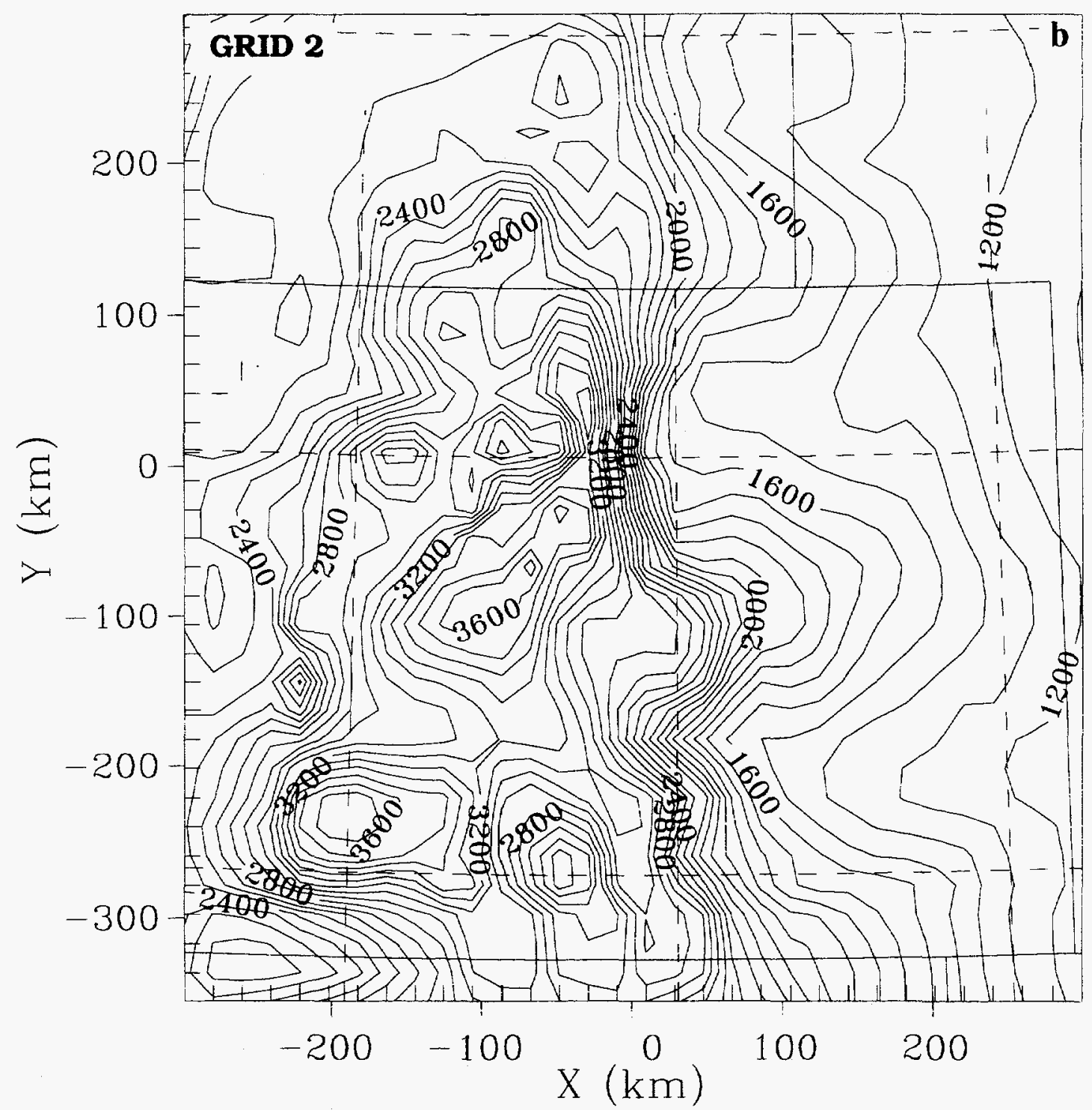

Figure 6.2 Continued. b) Grid 2, whose southwest corner is at $36.71^{\circ} \mathrm{N}, 108.69^{\circ} \mathrm{W}$, lat-lon increments in dashed lines of $2.5^{\circ}$, topographic contours every $100 \mathrm{~m}$.

katabatic flows, it is important to ensure that the sensitivity studies chosen will, indeed, extract that information. Stein and Alpert (1993) present a technique that formalizes the methodology for diagnosing the influence of various factors on a simulation. According to their analysis, when seeking to investigate the interaction of $n$ factors a series of $2^{n}$ simulations must be performed. Since we are interested in two factors, 1) katabatic flow (whose existence is dictated by the longwave radiation parameterization in this case) and, 2) mountain waves (caused by flow over topography as initialized and nudged with MAPS 

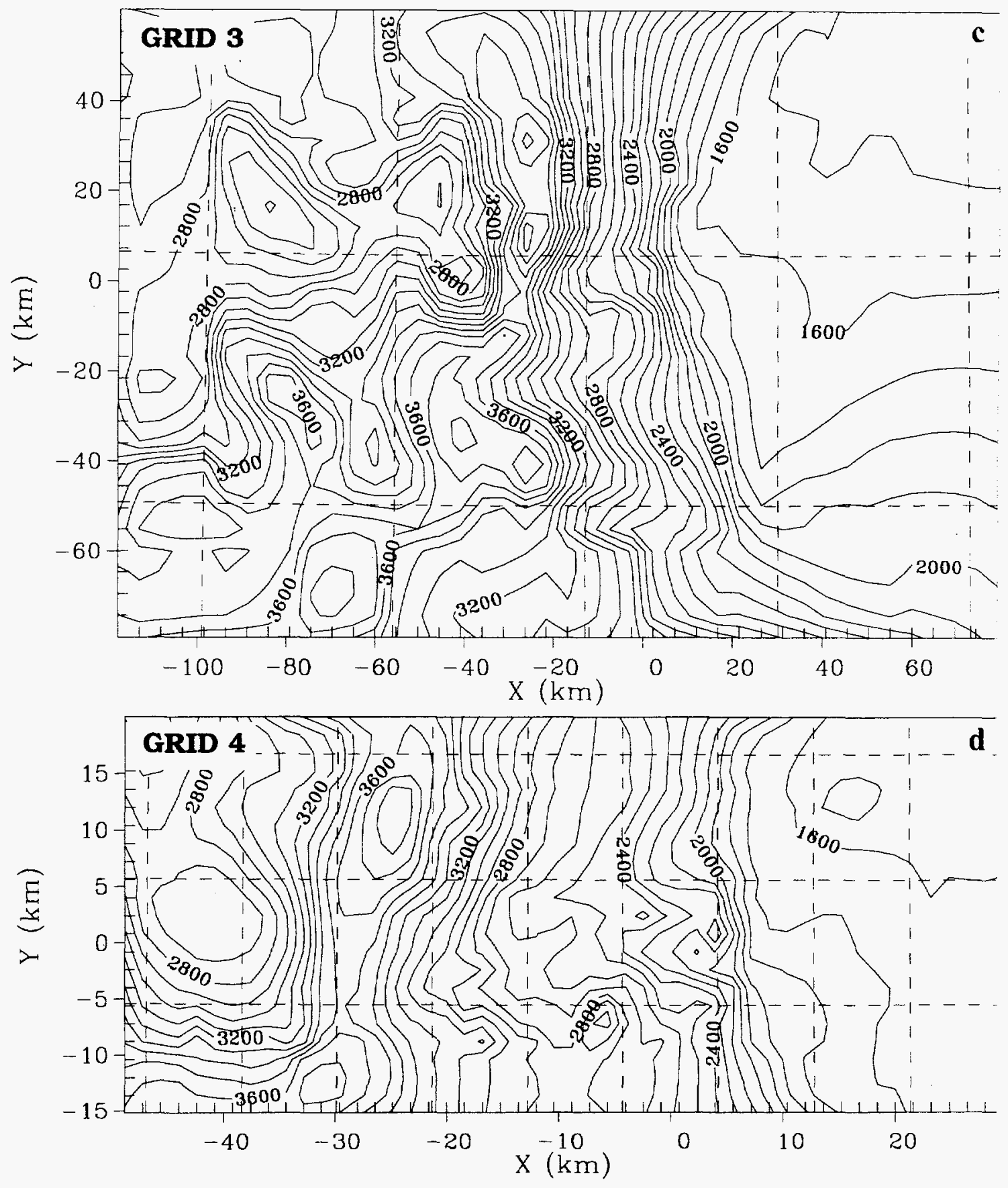

Figure 6.2 Continued. c) Grid 3, whose southwest corner is at $39.23^{\circ} \mathrm{N}, 106.72^{\circ} \mathrm{W}$, lat-lon increments (dashed lines) of $0.5^{\circ}$, d) Grid 4, whose southwest corner is at $39.81^{\circ} \mathrm{N}, 105.92^{\circ} \mathrm{W}$, latlon increment (dashed lines) of $0.1^{\circ}$. Topographic contours every $100 \mathrm{~m}$.

data), 4 simulations $\left(2^{2}\right)$ are required. It is shown below that due to the special nature of the simulations performed only three simulations are necessary for appropriate factor sep- 


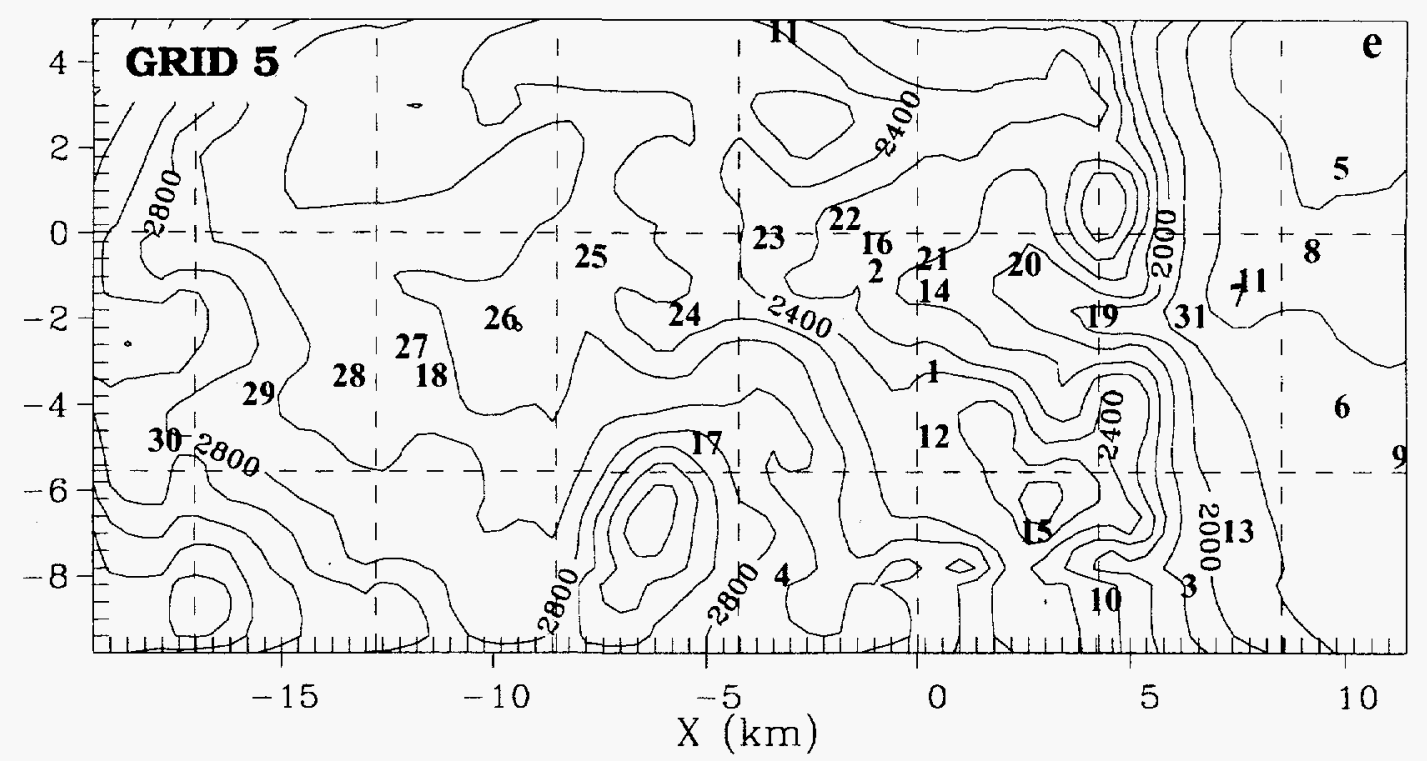

Figure 6.2 Continued. e) Grid 5, whose southwest corner is at $39.86^{\circ} \mathrm{N}, 105.58^{\circ} \mathrm{W}$, lat-lon increments (dashed lines) of $0.05^{\circ}$, topographic contours every $100 \mathrm{~m}$. Numbers 1-31 indicate where high time resolution (every $120 \mathrm{~s}$ ) meteorological variables and their forcings were extracted. aration.

According to Stein and Alpert (1993) the four simulations required are, 1) both MAPS nudging and the radiation parameterization active $\left.\left(f_{R N}\right), 2\right)$ the radiation parameterization active, but not the MAPS nudging ( $f_{\mathrm{R} 0}$, no initial winds), 3) the MAPS nudging only without the radiation parameterization active $\left(f_{0 N}\right)$ and 4$)$ neither MAPS nudging nor the radiation parameterization active $\left(\mathrm{f}_{00}\right)$. By definition then, the influence of these individual or combined factors on the meteorological conditions are:

$$
\begin{gathered}
\hat{f_{00}}=f_{00} \\
\hat{f}_{R 0}=f_{R 0}-f_{00} \\
\hat{f}_{0 N}=f_{0 N}-f_{00} \\
\hat{f}_{R N}=f_{R N^{-}}\left(f_{R 0}+f_{0 N}\right)+f_{00}
\end{gathered}
$$

The above equations are simplified greatly when one considers the meteorological impact of simulation $f_{00}$, without MAPS nudging and without the radiative parameteriza- 
tion active. Without MAPS nudging the winds and the factors that cause them (i.e. pressure gradient acceleration) are initially zero and conditions are initially horizontally homogeneous. The only way for winds to be produced when conditions are initially horizontally homogeneous without winds is by radiative forcing. However, $\mathrm{f}_{00}$, as required by Stein and Alpert (1993) must also have the radiation parameterization inactive. Therefore, $f_{00}$ will have zero winds for all time and the relevant equations become,

$$
\begin{gathered}
\hat{f_{00}}=0 \\
\hat{f}_{R 0}=f_{R 0} \\
\hat{f}_{0 N}=f_{0 N} \\
\hat{f}_{R N}=f_{R N}-\left(f_{R 0}+f_{0 N}\right)
\end{gathered}
$$

and the simulation $\mathrm{f}_{00}$ is irrelevant and unnecessary. We are therefore left with 3 simulations to perform which are, $\mathrm{f}_{\mathrm{R} 0}$, which will be referred to as the 'katabatic flow only' or 'KFO' case, $f_{0 N}$, referred to as the 'mountain wave only' or 'MWO' case, and $f_{R N}$, referred to as the 'realistic' or 'real' case. The discussion of these three cases follows below, beginning with a comparison of the realistic case to observations.

\subsection{COMPARISON OF THE REALISTIC CASE TO OBSERVATIONS}

\subsubsection{Large-scale}

As described in Chapter 3 and Appendix A this case night was dominated by clear skies as a consequence of the influence of a high pressure system over Arizona. A weak cold front was located in the Dakotas and Montana which set up geostrophic northwesterly flow aloft. The wind profiler at Gross Reservoir indicated $W$ to $N W$ winds over $10 \mathrm{~m} \mathrm{~s}^{-1}$ on the east side of the continental divide in the Eldorado Canyon drainage up to $5 \mathrm{~km}$ 
AGL. Based on averages in the $2000 \mathrm{~m}-4000 \mathrm{~m}$ MSL layer above the Grand Junction $00 \mathrm{Z}$ and $12 \mathrm{Z}$ soundings the Froude number for this case hovered around 0.45 overnight. A host of non-linear effects is therefore expected (see Section 2.6). The wind direction was consistently $319^{\circ}$ at both sounding times with an average wind speed of $8.6 \mathrm{~m} \mathrm{~s}^{-1}$ and 6.5 $\mathrm{m} \mathrm{s}^{-1}$ for $00 \mathrm{Z}$ and $12 \mathrm{Z}$, respectively. Within this same layer atmospheric stability dropped from $\partial \theta / \partial z=1.6 \mathrm{~K} \mathrm{~km}^{-1}$ (or $\partial T / \partial z=8.2^{\circ} \mathrm{C} \mathrm{km}^{-1}$ ) to $\partial \theta / \partial z=1.0 \mathrm{~K} \mathrm{~km}^{-1}$ (or $\partial T / \partial z=$ $8.8^{\circ} \mathrm{C} \mathrm{km}^{-1}$ ). Since the U.S. Standard Atmosphere has a stability of $\partial \theta / \partial z=3.4 \mathrm{~K} \mathrm{~km}^{-1}$ (or $\partial T / \partial z=6.5^{\circ} \mathrm{C} \mathrm{km}^{-1}$ ) clearly conditions of below average stability prevailed throughout this case night. One can anticipate that mountain wave flow could relatively easily penetrate very close to the surface, perhaps only being hindered by strong, near surface stable layers driven by nocturnal cooling. That this may well have been the case is indicated by the light winds that prevailed in the very lowest $10 \mathrm{~m}$ of the atmosphere. NCAR Mesonet, ASCOT experimental and NOAA ARL experimental towers all indicated wind speeds in the $2-5 \mathrm{~m} \mathrm{~s}^{-1}$ category (see Chapter 3 ).

Simultaneously the BAO tower, in Erie, Colorado about $20 \mathrm{~km}$ east of Boulder, indicated wind speeds varying around $5 \mathrm{~m} \mathrm{~s}^{-1}$ at higher levels (50-250 $\mathrm{m} \mathrm{AGL)}$, and around 3 $\mathrm{m} \mathrm{s}^{-1}$ at the $10 \mathrm{~m}$ level (Figure 6.3). Wind direction was easterly prior to the onset of katabatic flow, westerly in the early stages of katabatic flow, and gradually turned to the north and northeast by 0400 MST. The late night northward turn of the wind could be due to the slightly eastward shift of the lee trough location, causing the BAO to reside in geostrophic northerly forcing, or possibly a result of coriolis forcing. The timing of wind direction transitions was highly variable within the ASCOT network, however, and some areas, such as low levels in Coal Creek Canyon, experienced continuous westerly winds. 
This variability is seen in a time series of wind direction for the BAO tower for 4 atmospheric levels, shown in Figure 6.3b. Four relatively distinct stages have been delineated in the BAO data, as shown by thick, dashed vertical lines in panels $a$ and $d$. The first stage
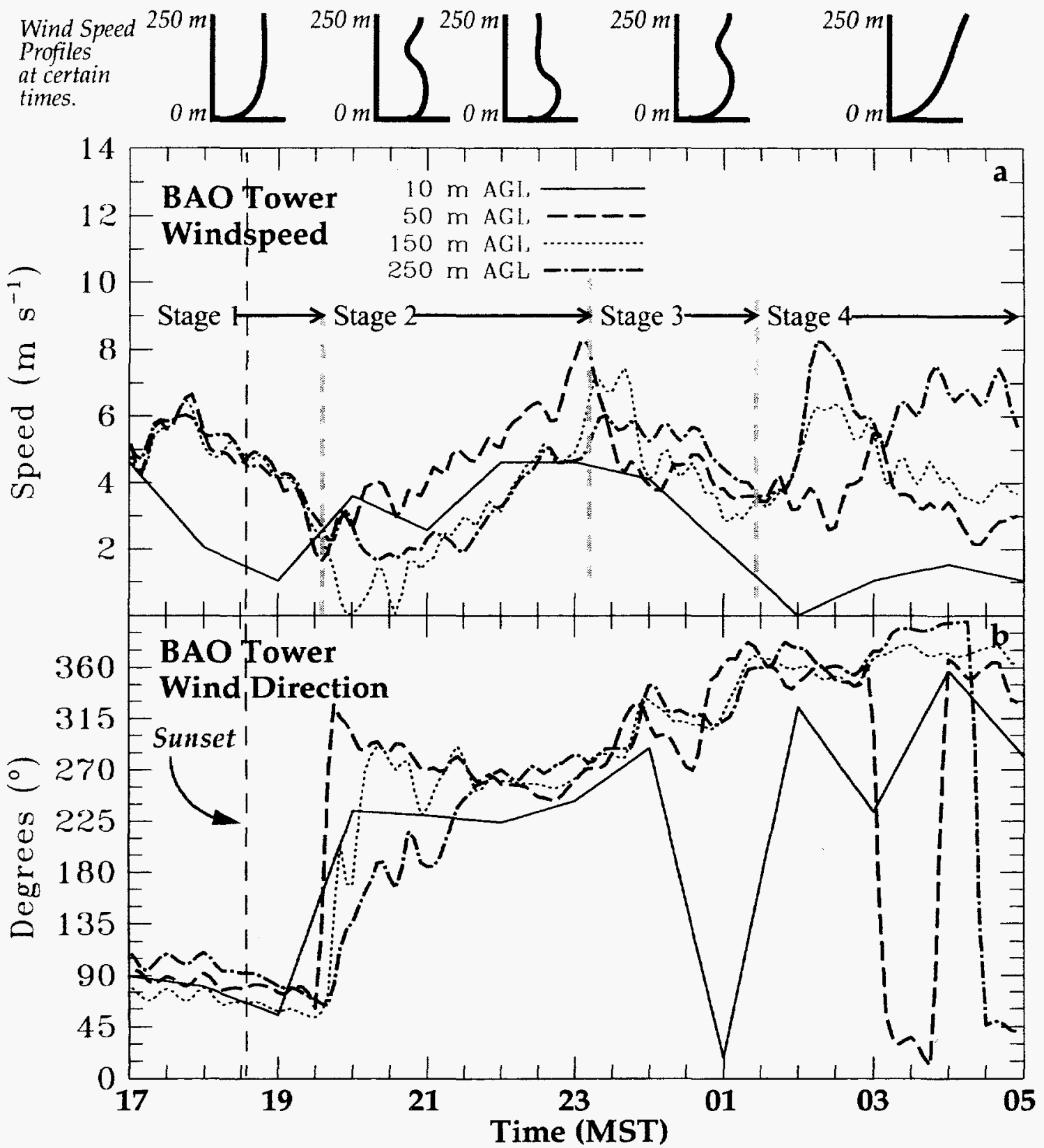

Figure 6.3 a) wind speed and b) direction, c) standard deviation of wind direction, and d) temperature, for the $300 \mathrm{~m}$ Boulder Atmospheric Observatory from 1700 MST 3 Sep 1993 to 0500 MST 4 Sep 1993 at 4 levels (as indicated). The $10 \mathrm{~m}$ level is from the Erie Mesonet site at the base of the tower and in 1 hour intervals. Note the number of different scales of variability and the 4 distinct stages delineated by thick, dashed vertical lines. Sunset is indicated by the thin, dashed line at 1839 MST. Vertical profiles of wind speed, based on the panel $a$ data, are shown at the top of the figure. 

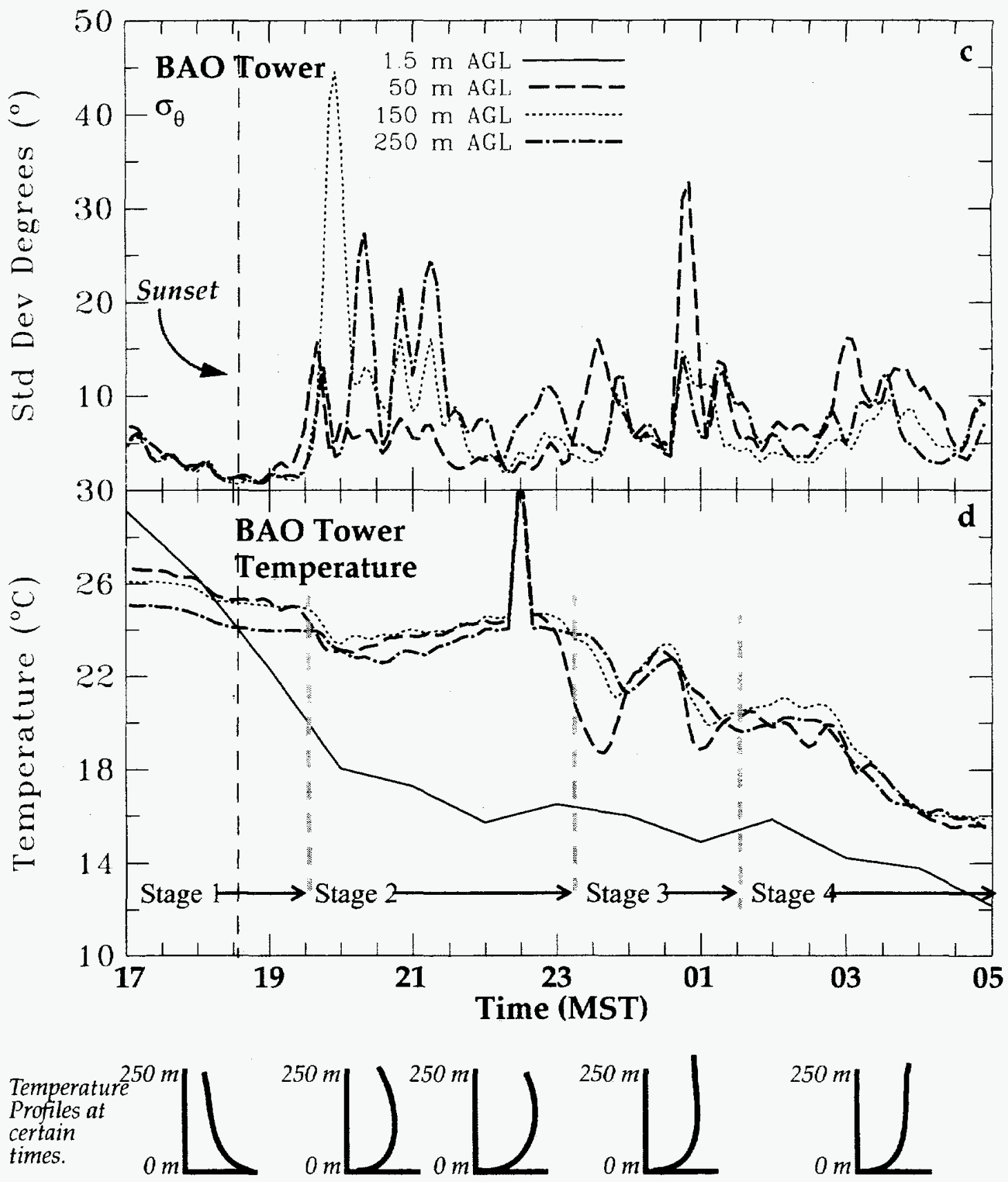

Figure 6.3 Continued. c) standard deviation of wind direction, and d) temperature. Vertical profiles of temperature at certain times are shown at the bottom of panel $d$.

represents a period of upslope flow - a remnant of daytime heating - during which wind speeds and temperatures dropped (panels $a$ and $d$ ), wind direction was consistently from the east (panel $b$ ), and $\sigma_{\theta}$ was low and invariant with height (panel $c$ ). After the typical pre-katabatic flow lull in wind speed at $\sim 1930$ MST, katabatic flow begins with an even- 
tual wind direction shift to the westerly component at all levels during the second stage. This wind direction shift occurs almost immediately at the two lowest levels but more gradually at upper levels, as can be expected for deepening katabatic flow. Directional shear is significant during this phase, which, along with a low level jet in the wind speed profile (see the drawings above panel a), causes heightened wind direction variability (panel $c$ ) until 2130 MST.

After $2130 \mathrm{MST}$, directional shear is reduced and $\sigma_{\theta}$ returns to relatively low values. Note that although $1.5 \mathrm{~m}$ level temperatures drop throughout this period, the temperatures at higher levels do not, so that by the end of the second stage ( 2330 MST) strong static stability resides below 50 $\mathrm{m}$ (see the 3rd temperature profile under Figure 6.3d). During the third stage (2315-0130 MST) wind direction shifts gradually to northerly, wind speeds decrease to half their initial value and $\sigma_{\theta}$ is variable. Interestingly, temperatures at $50 \mathrm{~m} \mathrm{AGL}$ and above decrease by $\sim 4^{\circ} \mathrm{C}$, while at $1.5 \mathrm{~m}$ the temperature drops only slightly. This appears to be caused by low-level shear that mixes temperatures effectively. Consequently, cooler air is gradually supplied to upper levels allowing temperatures to drop at $50 \mathrm{~m}, 150 \mathrm{~m}$ and $250 \mathrm{~m}$ AGL. Surface meteorological plots indicate that cold air advection was not a large cooling mechanism on this night. The cooling trend continues throughout the fourth stage from 0130 - 0500 MST as consistent north and north-northeasterly winds invade the upper levels. The resulting speed shear aloft (see the last wind speed profile above panel a) mixes cool air from lower levels to cause cooling in upper levels. Low-level katabatic flow and upper level ambient forcing appear indistinguishable at this time. Strong stratification at the lowest levels allows cooling to continue at the lowest measurement level during Stage 4.

Clearly, since the outer domain of Grid 1 was nudged with the MAPS data set, which incorporates most of the routinely available meteorological observations (see Section 3.2 .5 ), one expects 
the Grid 1 model solution from the realistic case to well represent the large scale observations for this case night. On Grids 2-5 where 4DDA is not active, however, such a comparison is not assured. Model output for comparison with the upper level synoptic conditions existing on this day corresponds well to the observed conditions aloft. Figures 6.4 and 6.5 , show the model results on a level horizontal surface for approximately the $500 \mathrm{mb}$ and $700 \mathrm{mb}$ levels, respectively, and though at different times, are comparable with Figures 3.2 and 3.3 with regards to synoptic characteristics. A plot such as this is not shown for $850 \mathrm{mb}$ because this level intersects the ground surface over the majority of the domain. At $5500 \mathrm{~m} \mathrm{MSL}$ (Figure 6.4), the model clearly indicates relatively high pressure to the southwest and northwesterly geostrophic flow aloft, as expected from the observations (see Figure 3.2). At both 2100 MST and 0200 MST there is only a small indication of lee trough effects over Colorado. Wind speed and direction are consistent for both times. At $\sim 3000 \mathrm{~m}$ MSL (Figure 6.5), a significant lee trough exists clearly indicated by the southward bend in the isobars over Colorado for both times. Wind speed and direction is more variable at this level. Over the ASCOT study region the wind direction changes $180^{\circ}$ between 2100 MST and 0200 MST due to an eastward shift in the lee trough location later in the overnight period.

\subsubsection{Comparisons with ASCOT observations.}

Table 6.4 shows the ASCOT observational sites and the RAMS model grid point nearest to that data point. Information at these model data points were extracted from either Grid 5 or Grid 4, as appropriate, in 2 minute intervals (every two long timesteps) to facilitate comparisons with observations of varied averaging intervals. Tower data was generally available in hourly intervals, sodar data in 15 minutes averages, and wind profiler data in 

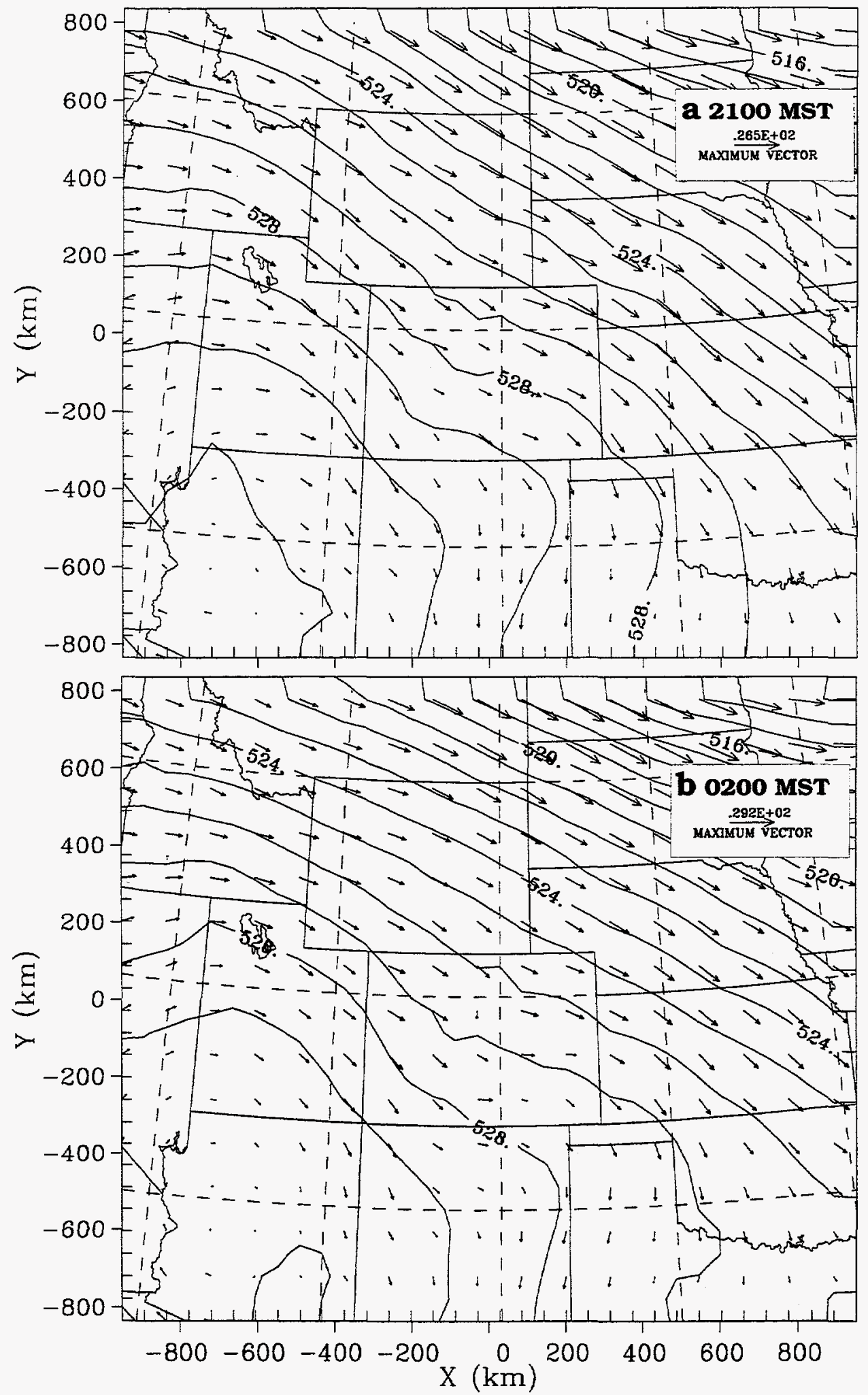

Figure 6.4 Wind vectors and pressure ( $1.0 \mathrm{mb}$ increments) on a horizontal plane $5518 \mathrm{~m}$ MSL for a) 2100 MST, 3 Sep 1993 and b) 0200 MST, 4 Sep 1993. Lee troughing is very weak compared to the $700 \mathrm{mb}$ flow in Figure 6.5 . 

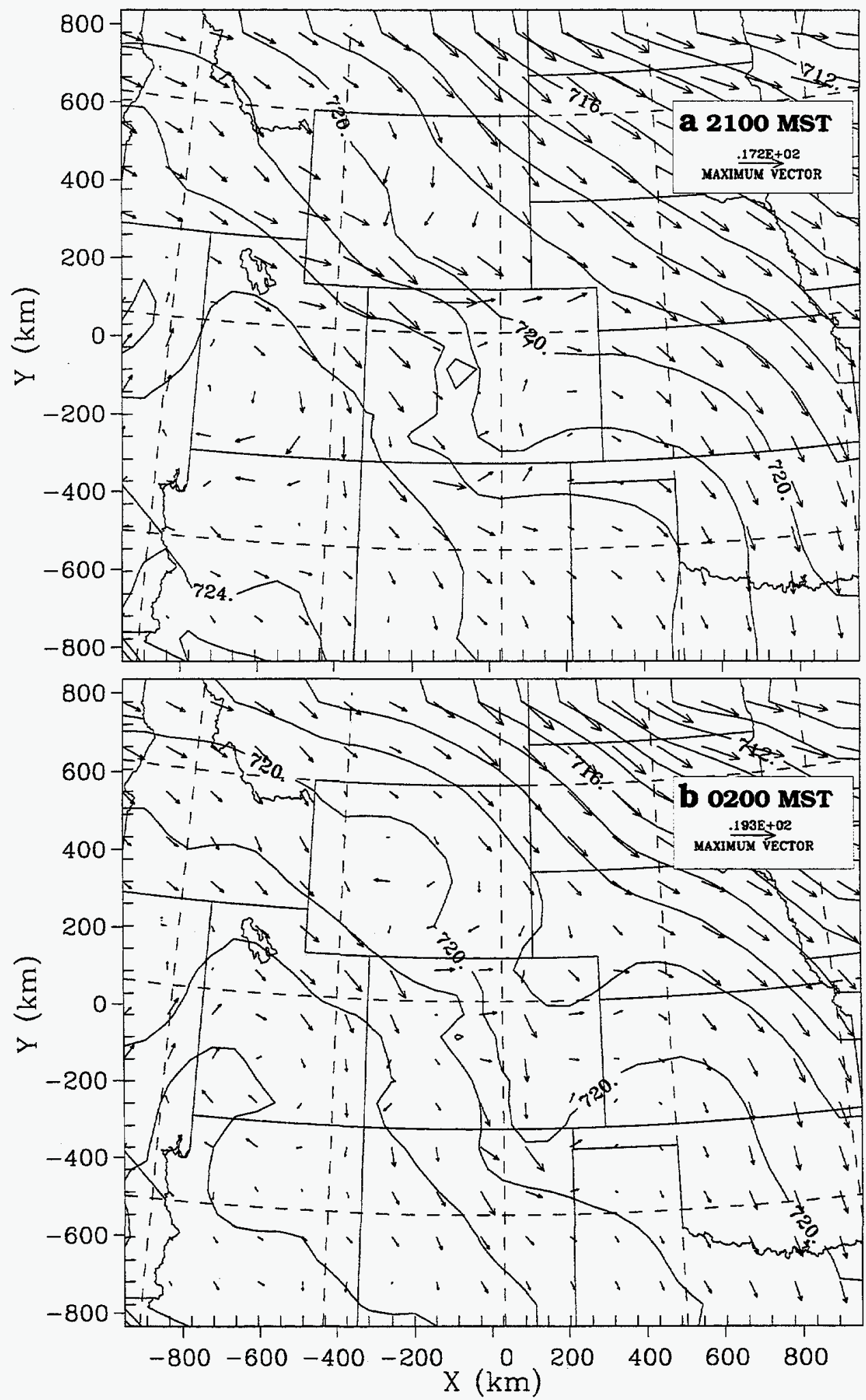

Figure 6.5 Wind vectors and pressure ( $1.0 \mathrm{mb}$ increments) on a horizontal plane $2973 \mathrm{~m}$ MSL for a) 2100 MST, 3 Sep 1993 and b) 0200 MST, 4 Sep 1993. Lee troughing is evident over Colorado indicative of strong mountain forcing on the incoming northwesterly flow. 
Table 6.4: ASCOT 1993 observational sites used for comparisons with RAMS output, nearest grid point and distance to site. ${ }^{*}$ - sites where model terrain height differs significantly from that of observational site. Bold, italicized sites have features most conducive to reliable comparison. gpt $=$ grid point, Elev $=$ elevation and Dist $_{H}=$ horizontal distance.

\begin{tabular}{|c|c|c|c|c|c|c|c|c|c|}
\hline ID & Name of site & Elev & Lon & Lat & Grid & Near & Near & Elev $\left(\Delta_{\text {elev }}\right)$ & Dist $_{H}$ to \\
\hline & & $(\mathrm{m})$ & $\left({ }^{0}\right)$ & $\left(^{\circ}\right)$ & \# & I gpt & $J \mathrm{gpt}$ & $(\mathrm{m})$ & $\mathrm{gpt}(\mathrm{m})$ \\
\hline & \multicolumn{9}{|l|}{ Profilers } \\
\hline $\mathrm{BA}$ & $B A O$ & 1577 & -105.00 & 40.05 & 4 & 50 & 17 & $1576(-1)$ & 619.9 \\
\hline \multirow[t]{2}{*}{ GR } & Gross Reservoir & 2280 & -105.36 & 39.95 & 5 & 47 & 24 & $2270(-10)$ & 147.9 \\
\hline & \multicolumn{9}{|l|}{ Sodars } \\
\hline $\mathrm{CC}$ & Coal Creek* & 1996 & -105.28 & 39.88 & 5 & 65 & 5 & $2115(+119)$ & 201.7 \\
\hline IS & Indiana St. & 1716 & -105.17 & 39.88 & 4 & 41 & 5 & $1764(+48)$ & 296.8 \\
\hline MI & Militzer & 1657 & -105.24 & 39.97 & 5 & 73 & 29 & $1688(+31)$ & 143.2 \\
\hline NR & NREL/WETC & 1847 & -105.23 & 39.91 & 5 & 75 & 15 & $1836(-11)$ & 165.0 \\
\hline $\mathrm{RO}$ & Robinson & 1743 & -105.27 & 39.93 & 5 & 67 & 21 & $1794(+51)$ & 172.7 \\
\hline \multirow[t]{2}{*}{$\mathrm{RU}$} & Ruthrauff & 1713 & -105.24 & 39.94 & 5 & 72 & 23 & $1746(+33)$ & 107.8 \\
\hline & \multicolumn{9}{|l|}{ Towers } \\
\hline $\mathrm{BA}$ & $B A O 300 \mathrm{~m}$ & 1577 & -105.00 & 40.05 & 4 & 50 & 17 & $1576(-1)$ & 619.9 \\
\hline RFP & Rocky Flats $61 \mathrm{~m}$ & 1849 & -105.22 & 39.90 & 5 & 77 & 11 & $1842(-7)$ & 168.2 \\
\hline LRH & Linda's Ranch $10 \mathrm{~m} *$ & 2003 & -105.28 & 39.88 & 5 & 65 & 5 & $2115(+112)$ & 201.7 \\
\hline PPK & Paul's Peak $10 \mathrm{~m}^{*}$ & 2472 & -105.30 & 39.87 & 5 & 60 & 3 & $2365(-107)$ & 126.6 \\
\hline RRH & Rex Ranch $10 \mathrm{~m}$ & 1705 & -105.26 & 39.94 & 5 & 69 & 22 & $1756(+51)$ & 182.4 \\
\hline RRK & Rim Rock $10 \mathrm{~m}^{*}$ & 2330 & -105.35 & 39.90 & 5 & 50 & 12 & $2400(+70)$ & 105.8 \\
\hline PFT & Pat's Flat $10 \mathrm{~m}$ & 1874 & -105.24 & 39.86 & 5 & 73 & 1 & $1925(+51)$ & 286.1 \\
\hline LRH & Lois' Ranch $10 \mathrm{~m}$ & 2015 & -105.27 & 39.89 & 5 & 66 & 8 & $2043(+28)$ & 177.8 \\
\hline GRD & Gross Dam $10 \mathrm{~m}^{*}$ & 2085 & -105.35 & 39.94 & 5 & 50 & 21 & $2220(+135)$ & 210.3 \\
\hline $\mathrm{RO}$ & Robinson $10 \mathrm{~m}$ & 1743 & -105.27 & 39.93 & 5 & 67 & 21 & $1794(+51)$ & 172.7 \\
\hline GR & Gross Reservoir $10 \mathrm{~m}$ & 2280 & -105.36 & 39.95 & 5 & 47 & 24 & $2270(-10)$ & 147.9 \\
\hline NR & NREL/WETC $10 \mathrm{~m}$ & 1847 & -105.23 & 39.91 & 5 & 75 & 15 & $1836(-11)$ & 165.0 \\
\hline $\mathrm{BOU}$ & Boulder Mesonet* & 1612 & -105.25 & 40.01 & 4 & 36 & 14 & $1741(+129)$ & 591.1 \\
\hline ROL & Rollinsville Mesonet & 2750 & -105.48 & 39.92 & 5 & 21 & 17 & $2669(-18)$ & 165.3 \\
\hline ERI & Erie Mesonet & 1585 & -105.00 & 40.05 & 4 & 50 & 17 & $1576(-9)$ & 619.9 \\
\hline
\end{tabular}

half-hourly intervals. At a number of sites in Table 6.4, we find that the elevation differ-

ence between the instrument location and the nearest model grid point is well over $50 \mathrm{~m}$.

These sites have subjectively been deemed less suitable for observational comparison and 
will not be considered in the following discussion. Note also that only those instrument sites whose location fell within $1 \mathrm{~km}$ or less of a model grid point were included in Table 6.4. For instruments in the mountains a far more strict criteria should be used, such as, perhaps, at most a $200 \mathrm{~m}$ distance from instrument to model grid point. As a general rule, model to observational comparisons were best on the innermost grid, Grid 5, due to the fine horizontal grid spacing there. Consequently, the following comparisons concentrate on the region of interest around Eldorado Canyon, which is mostly encompassed by Grid 5.

It is important to consider one's expectations when comparing model results to observations. Ideally, we would like to have exact representation of atmospheric conditions. This expectation is unrealistic, particularly in complex terrain, for a number of reasons. First and foremost model data at grid points represents conditions for a certain grid volume whereas tower data represents a particular atmospheric point. Second, because the model cannot be run with infinite resolution, inherent scale filtering of model data is present, whereas the atmospheric data contains contributions from all ranges of scales. Complex topography presents another difficulty. For a given grid spacing, in this case as small as $400 \mathrm{~m}$ horizontally, and smoothing, the resolved topographic features can vary significantly enough to cause comparison difficulty. In addition to the distance to the instrument location from the nearest grid point (last column in Table 6.4), the actual terrain height in the model may differ significantly from that of the actual instrument site (number in parentheses, next to last column in Table 6.4). In cases where elevation differences are less than $\sim 20$ meters it is likely that most of the terrain features significant to thermally-forced circulations are captured. Where this terrain difference exceeds $\sim 100 \mathrm{~m}$, however, it is 
likely that the terrain representation is locally inadequate to expect reasonable meteorological comparison particularly at low levels AGL (these sites marked with $\mathrm{a}^{*}$ ). Interpolation between the 4 model grid points surrounding a site is one way to mitigate some of the comparison problems, but this method can only overcome certain aspects of misrepresented terrain. In the following comparisons, interpolation was not done because extracting that much model data for each site (versus 1 point per site) exceeded local storage capability. Only those instrument locations reasonably close (subjectively defined based on surrounding topography) were used. The most appropriate sites for model/observational comparisons are shown in bold italics in Table 6.4.

\section{Profilers}

Gross Reservoir profiler observations and realistic case model output for the grid point nearest Gross Reservoir are shown in Figures 6.6 and 6.7, respectively, for comparison purposes (the BAO profiler was not operational until 13 Sep 1996). The observed timeheight profile in Figure 6.6 compares reasonably with the modeled profile although generally the model overestimates wind speeds by $1-5 \mathrm{~m} \mathrm{~s}^{-1}$. In particular, the observed profile contains the following features, strong $12-15 \mathrm{~m} \mathrm{~s}^{-1}$ west to southwesterly flow all the way to the lowest observation level ( $123 \mathrm{~m}$ AGL) except for the $0030-0230$ period of lesser flow from the east and southeast; an intrusion of southwesterly $\sim 15 \mathrm{~m} \mathrm{~s}^{-1}$ winds from 2030 to 2200 MST and again from 2300-0000 MST; and between these intrusions a period of weaker winds under $12 \mathrm{~m} \mathrm{~s}^{-1}$. The model results are in poor comparison during the early 'spin-up' stage $(\sim 1700-1815 \mathrm{MST})$ but indicate two intrusions of strong winds that correspond well to the observed intrusions (the latter intrusion being $>18 \mathrm{~m} \mathrm{~s}^{-1}$ or $\sim 3 \mathrm{~m} \mathrm{~s}^{-1}$ too strong). The period of lighter winds centered on $2130 \mathrm{MST}$ is also captured. The pri- 
ASCOT Gross Reservoir Profiler 09/03-04/93
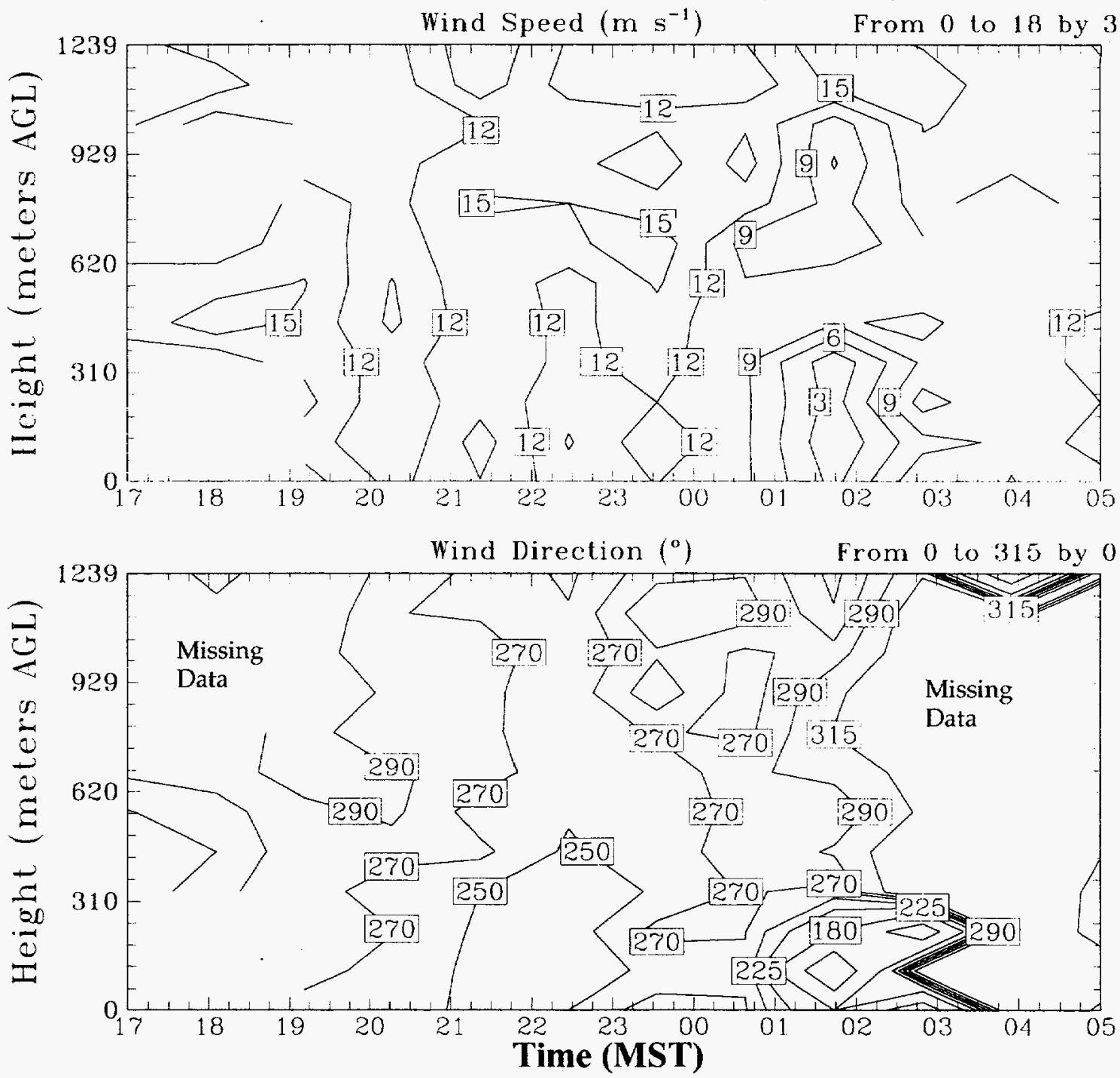

Figure 6.6 Observed wind speed and direction for the Gross Reservoir profiler approximately $15 \mathrm{~km}$ west into Eldorado Canyon. Strong westerly winds mix downward from 2030 to 2200 MST and again from 2300-0000 MST. Compare with Figure 6.7..

mary model failure is in simulating the low-level easterly wind period just prior to the degradation in observational quality (0300-0600 MST)

\section{Sodars}

Observed sodar profiles of winds from this case day are presented in Figure 6.8 (Militzer) and Figure 6.10 (Robinson) whereas the corresponding profiles extracted from the model solution are in Figure 6.9 and Figure 6.11, respectively. It is clear that the sodars 


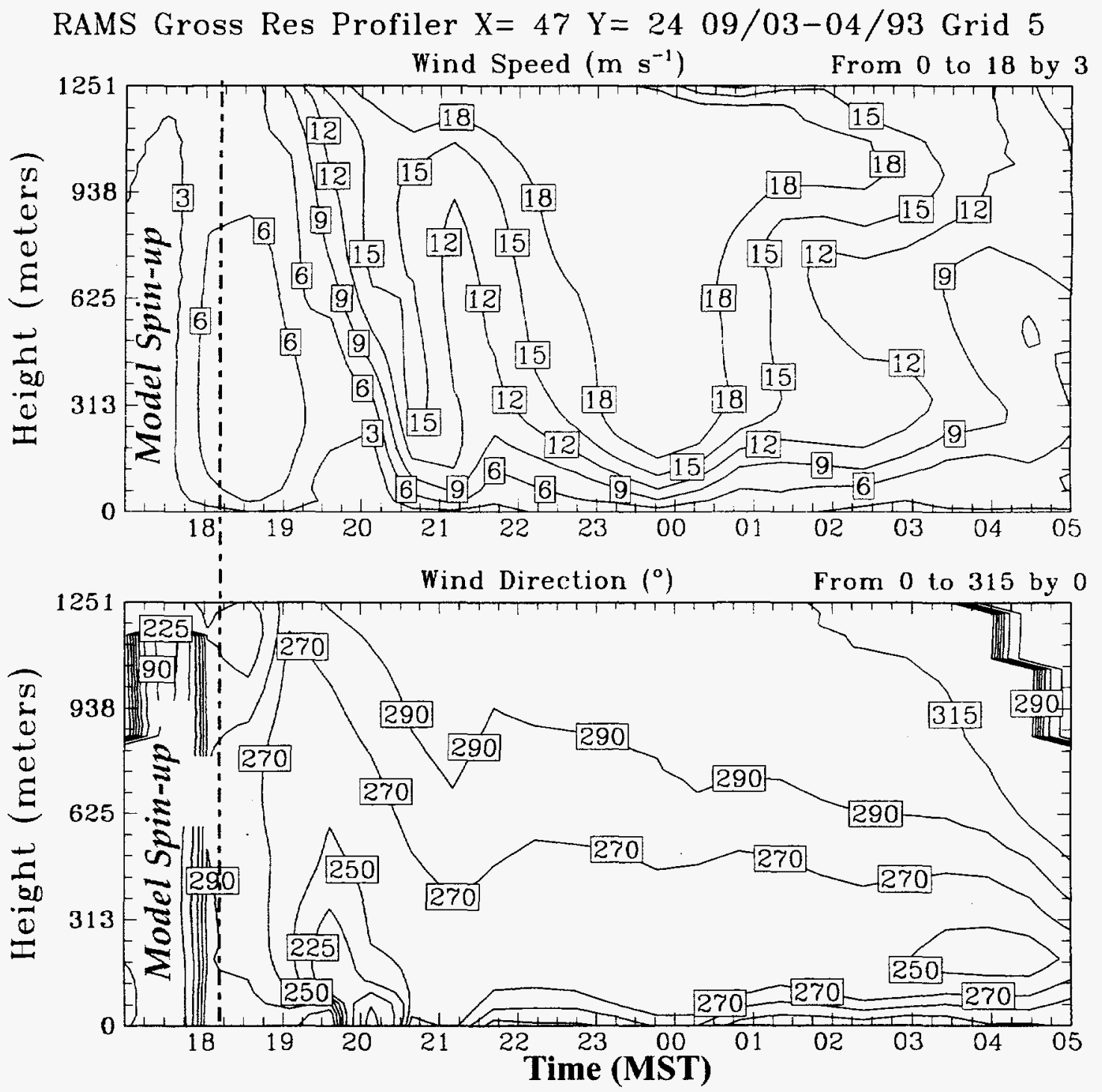

Figure 6.7 RAMS modeled wind speed and direction for the Gross Reservoir profiler location. Strong westerly winds mix downward from 2030 to 2130 MST and again from 2300 to 0100 MST somewhat similar to observations in Figure 6.6.

also suffered from some data acquisition problems. The Coal Creek Canyon sodar was

considerably more reliable than the others, but the narrow confines of that canyon pre-

clude resolving its terrain features sufficiently at the grid spacing chosen. Some useful

comparisons are able to be made with some of the remaining sodars, however.

The Militzer sodar observations show that westerly component flow - as would be expected drainage flow - dominated in the lowest 100 meters AGL until 0200 MST. During this period speeds varied as follows $2-4 \mathrm{~m} \mathrm{~s}^{-1}$ from 1800-2000 LST, $4-6 \mathrm{~m} \mathrm{~s}^{-1}$ from 
ASCOT Militzer Sodar 09/03-04/93

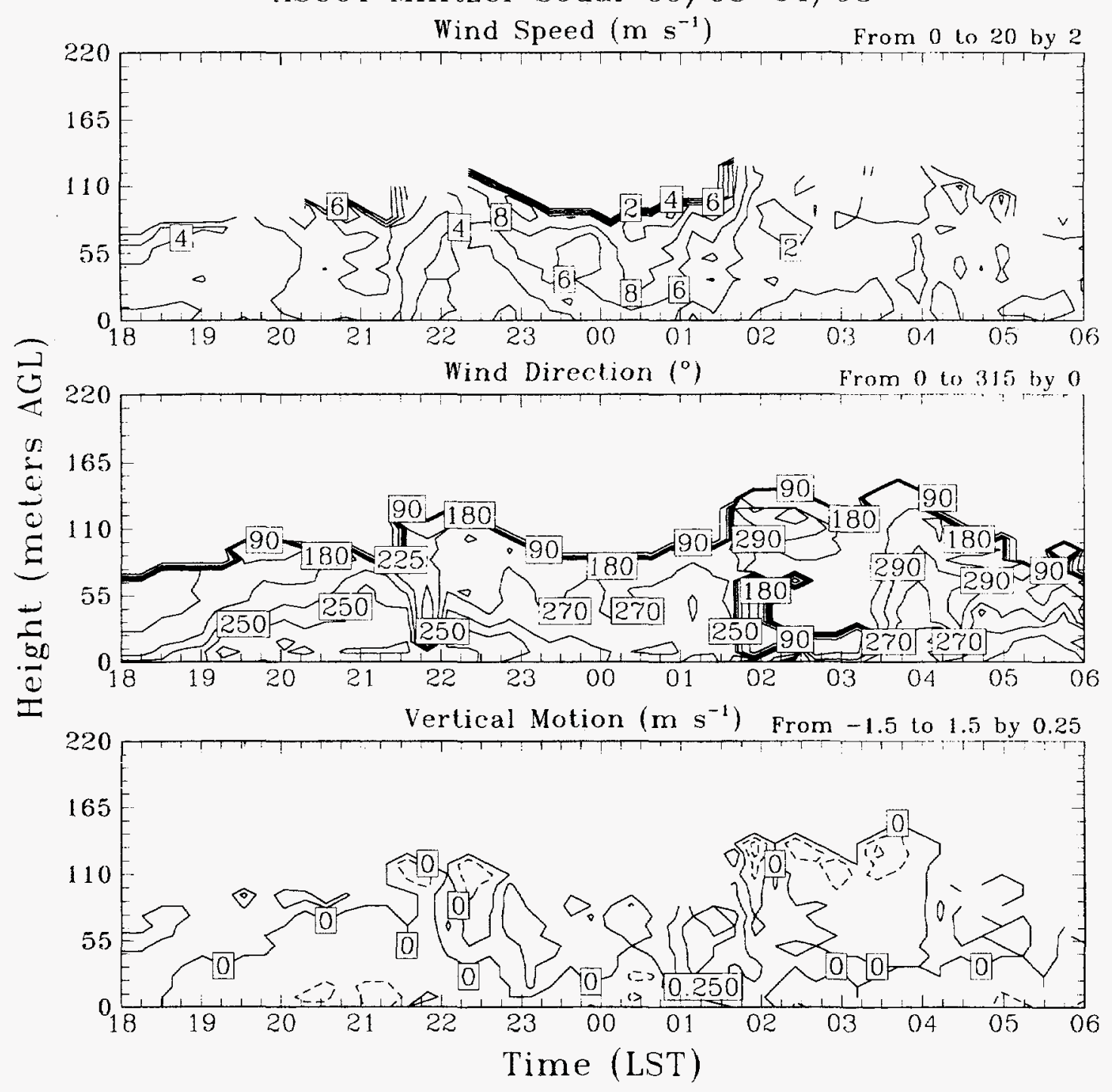

Figure 6.8 a) wind speed, b) wind direction and c) vertical motion as observed by the Militzer sodar for the overnight period 3-4 Sep 1993. Poor data is found above $110 \mathrm{~m}$ AGL. Compare with Figure 6.9.

2000-2130 MST, 2-4 $\mathrm{m} \mathrm{s}^{-1}$ from 2130-2300 MST and 6-8 $\mathrm{m} \mathrm{s}^{-1}$ from 2330-0130 MST.

After $0200 \mathrm{MST}$ a short increase in easterly flow is associated with a local deceleration to 2-3 $\mathrm{m} \mathrm{s}^{-1}$, until westerly flow at $4 \mathrm{~m} \mathrm{~s}^{-1}$ again dominates. Vertical motions were typically weak and variable, but generally negative. During the easterly incursion, a short period of upward vertical motion near $0.25 \mathrm{~m} \mathrm{~s}^{-1}$ occurred. The modeled vertical profile of winds in Figure 6.9 reveals similar features but with complete vertical coverage. The evolution of 


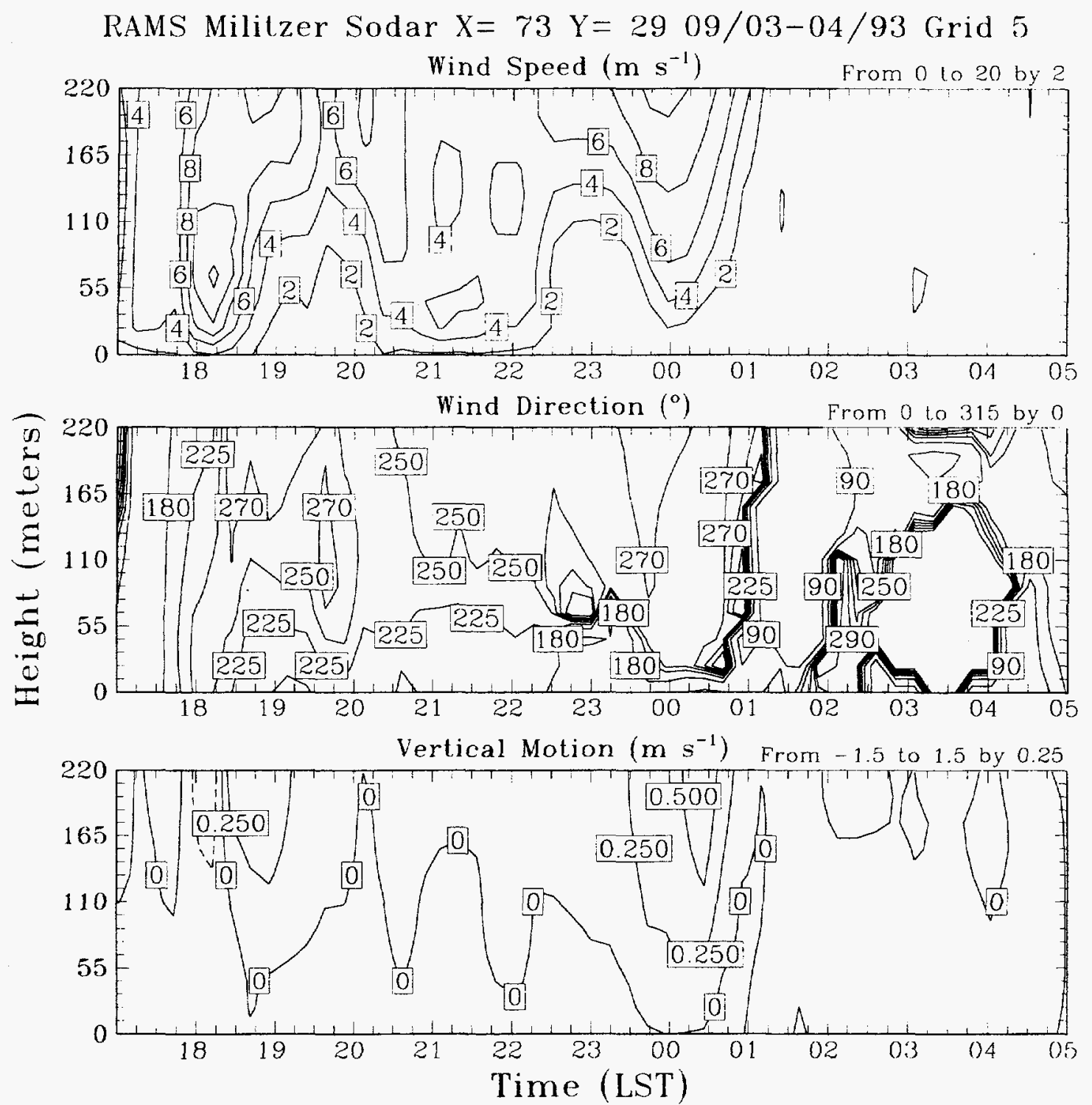

Figure 6.9 a) wind speed, b) wind direction and c) vertical motion as modeled by RAMS for a location near the Militzer sodar for the overnight period 3-4 Sep 1993. Compare with Figure 6.8 .

modeled wind direction indicates southwesterly to westerly flow at 2-4 $\mathrm{m} \mathrm{s}^{-1} 1830-2030$ MST, 4-6 $\mathrm{m} \mathrm{s}^{-1}$ from 2030-2215 MST, $2 \mathrm{~m} \mathrm{~s}^{-1}$ or less $2230-2330$, variable with height up to $8 \mathrm{~m} \mathrm{~s}^{-1}$ around midnight and $2 \mathrm{~m} \mathrm{~s}^{-1}$ thereafter somewhat like the observations. Also like the observations, the weakened flow is associated with an easterly surge of winds (the model begins this surge earlier). Westerly flow does return at the correct time but is weaker than observed. Given the uncertainties mentioned in the introduction to this sec- 


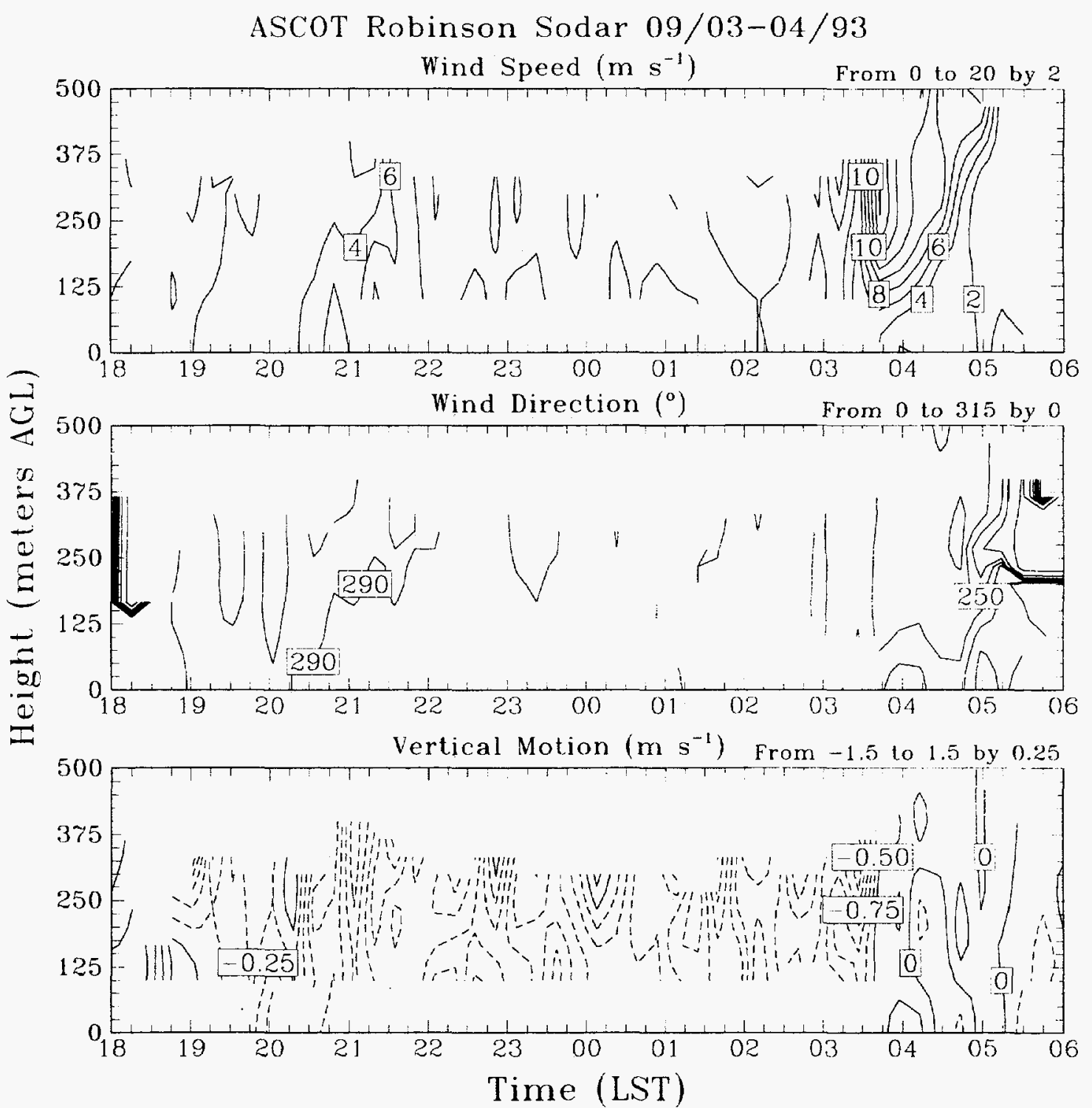

Figure 6.10 a) wind speed, b) wind direction and c) vertical motion as observed by the Robinson sodar for overnight 3-4 Sep 1993. Compare with the model results in Figure 6.11.

tion, the level of correspondence between the model and observations is good.

The observations by the sodar at site Robinson are somewhat more corrupted than those from the Militzer sodar, but some information can be garnered for comparison purposes. As shown in Figure 6.10 winds were generally $\sim 4 \mathrm{~m} \mathrm{~s}^{-1}$ and southwesterly to westerly until 2000 MST when wind turned somewhat northwesterly and accelerated to $7-10 \mathrm{~m} \mathrm{~s}^{-1}$. An intrusion of westerly winds up to $10 \mathrm{~m} \mathrm{~s}^{-1}$ occurred near 0400 MST. The model profile in Figure 6.11 does not show a lower wind speed period prior to 2000 MST but this time 
RAMS Robinson Sodar $X=67 \mathrm{Y}=2109 / 03-04 / 93$ Grid 5
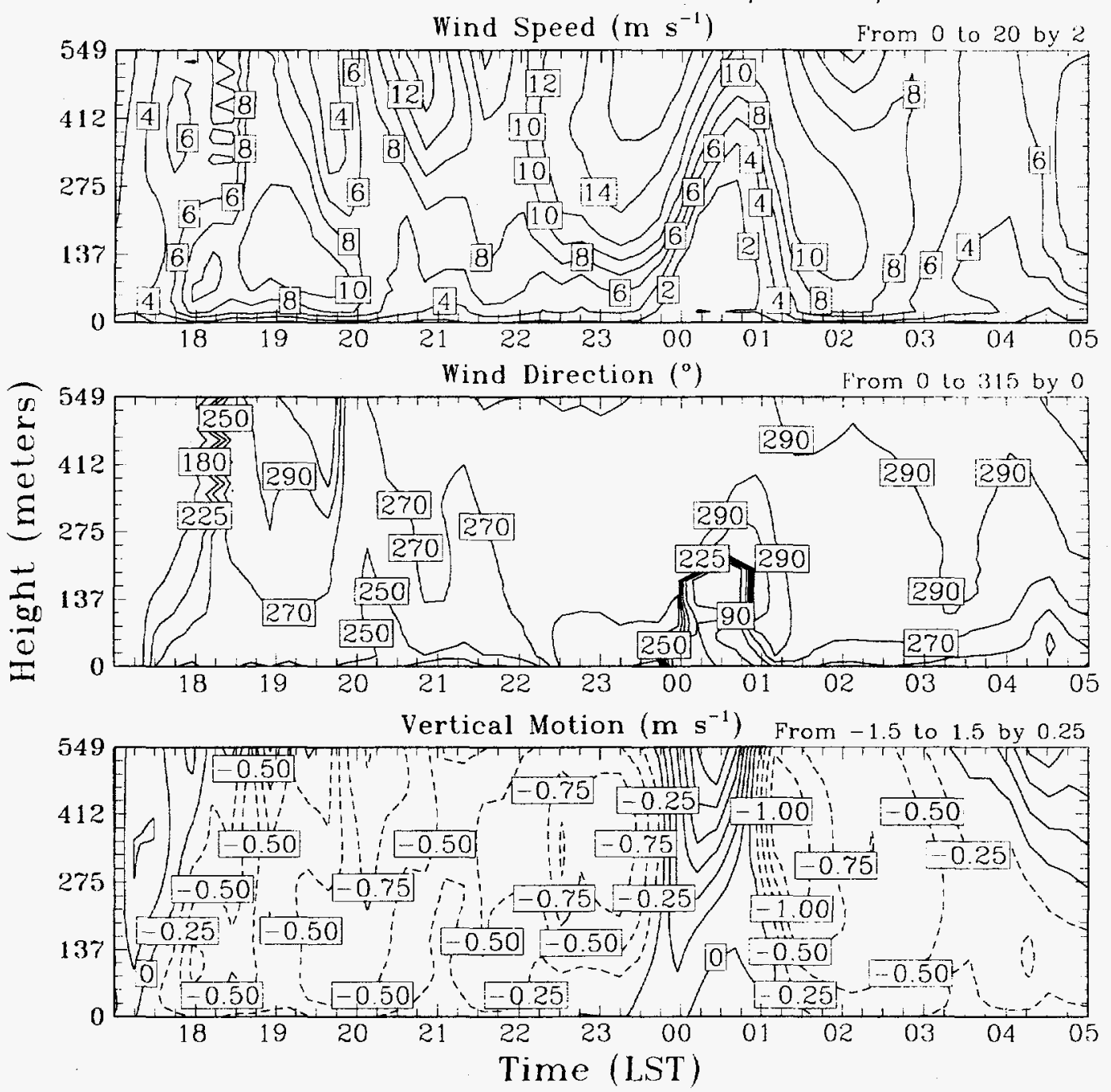

Figure 6.11 a) wind speed, b) wind direction and c) vertical motion as modeled by RAMS for a location near the Robinson sodar for the overnight period 3-4 Sep 1993. Compare with Figure 6.10 .

period is still influenced somewhat by model adjustment. After $2000 \mathrm{MST}$, the modeled winds are similar in strength to the observations, although a relatively strong westerly burst occurs near 2300 MST (sodar data is limited at this time) and the wind direction does not turn northwesterly until 2200 MST. A westerly acceleration of the flow to $10 \mathrm{~m} \mathrm{~s}^{-1}$ is modeled around 0200 MST, approximately 2 hours earlier than observed. Similar to the Militzer model profile, but unlike observations, a calm period of winds associated with a wind shift to easterly occurs just after midnight. Given the strength of the mountain wave 
flow on this case night the easterly flow is likely to have been caused by overturning, or rotor activity, in the lee. In support of this view the vertical flow in panel $c$ indicates a short period of upward vertical motion at this time. Convergence of the easterly flow with dominant westerly flow would cause upward vertical motion. In fact, since westerly flow lies above the easterly flow rotor processes are suggested. Vertical cross-sections of TKE, wind vectors and potential temperature indicate that weak rotor activity was occurring (not shown).

Model data from the NREL and Ruthrauff sodars (Figure 6.12 and Figure 6.13, respectively) verifies the variability observed and modeled for the previously described sodars. In particular the NREL sodar indicates winds with a westerly component at speeds that alternate from $\sim 4 \mathrm{~m} \mathrm{~s}^{-1}$ up to $\sim 10 \mathrm{~m} \mathrm{~s}^{-1}$. Stronger bursts of wind are accompanied by either upward or downward vertical motion and occur with a frequency of approximately every 4 hours. Vertical profiles of potential temperature and TKE from the model clearly indicate the upper level influence of mountain wave flow (not shown), particularly during the period 2000-0100 LST. After 0100 LST consistently building stable layers are found at the lower levels such that katabatic forcing again exists (though never completely free of mountain wave influence). The modeled Ruthrauff sodar data (Figure 6.13) also shows mountain wave induced variability but has a period of low-level katabatic flow from 18002000 MST. During this time a stable atmospheric stratification is able to develop up to 200 $\mathrm{m}$ AGL with radiative flux divergence equal to $-0.1 \mathrm{~K} \mathrm{hr}^{-1}$. After approximately 2100 MST surges of westerly mountain wave flow with high levels of turbulent kinetic energy mix through the stable layers to near the surface. The resulting weaker stratification is conducive to mountain wave flow domination until approximately $0200 \mathrm{MST}$. At this time 


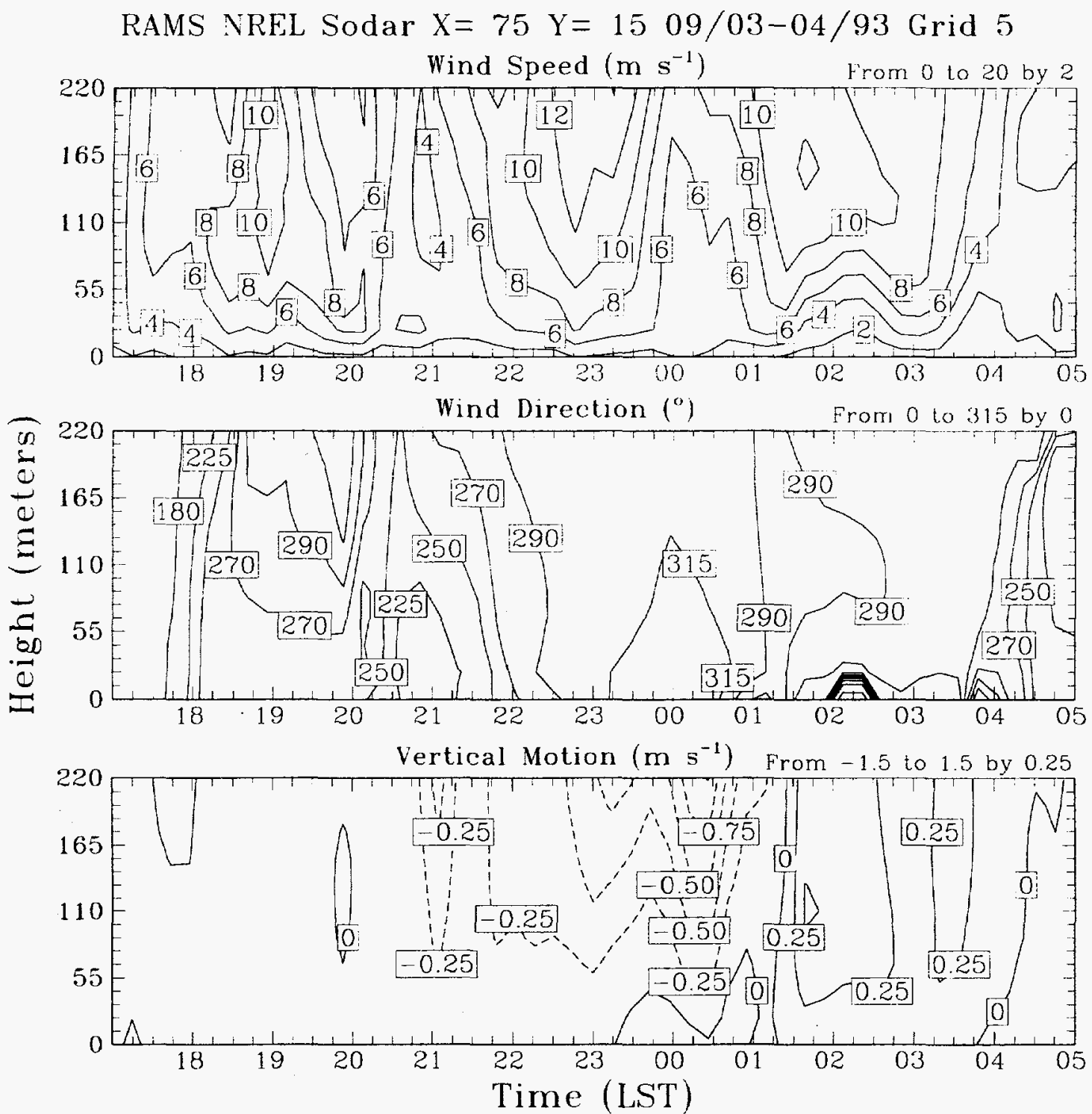

Figure 6.12 a) wind speed, b) wind direction and c) vertical motion as modeled by RAMS for a location near the NREL sodar for the overnight period 3-4 Sep 1993. Observed profiles do not contain sufficient information for comparison.

low level radiative cooling rates of up to $-0.3 \mathrm{~K} \mathrm{hr}^{-1}$ rebuild the stratification and prevent

mountain wave flow from dominating. A second short period of katabatic flow with a

poorly defined jet is able to develop from 0300-0500 MST before the simulation ends.

In summary, the modeled sodar profiles compare reasonably to observations, indicate

mountain wave influence, and are wrought with large horizontal and vertical variability.

Though the model performance is not ideal, confidence in the model capability is gained

by noting that a number of features similar to realistic complex terrain flow, such as rotor 
RAMS Ruthrauff Sodar $X=72 Y=2309 / 03-04 / 93$ Grid 5
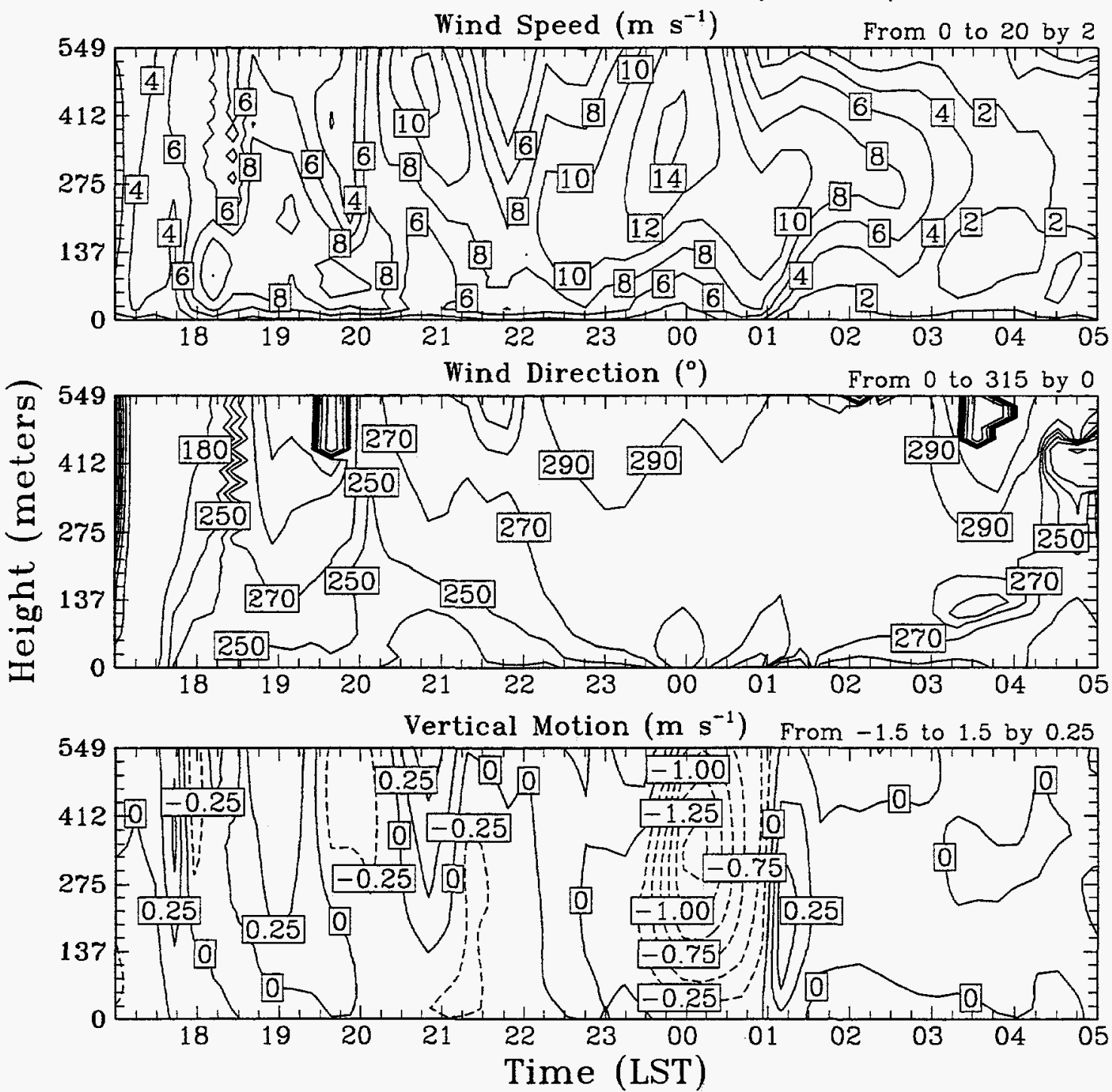

Figure 6.13 a) wind speed, b) wind direction and c) vertical motion as modeled by RAMS for a location near the Ruthrauff sodar for the overnight period 3-4 Sep 1993. Observed profiles do not contain sufficient information for comparison.

activity, katabatic flow jets, occasional flow intrusions and rapid wind direction changes,

occur throughout the simulation.

\section{Towers}

Although not as revealing as time-height cross sections of flow fields, tower data contains otherwise missing temperature data for comparisons. As a general statement, the wind speed and direction behavior of the model was as variable as described earlier for the sodars and profilers (see below). The Lois Ranch site, shown in Figure 6.14, was modeled 
well in some regards and poorly in others. After the first couple hours of model spin-up the wind speed is underestimated by the simulation and never really recovers from the initial errors. Based on the 1 hour value, these errors are not due to faulty initialization.Wind direction is modeled well throughout the night, despite being somewhat more variable than observed. The temperatures are underestimated by $2-5^{\circ} \mathrm{C}$, which may explain the

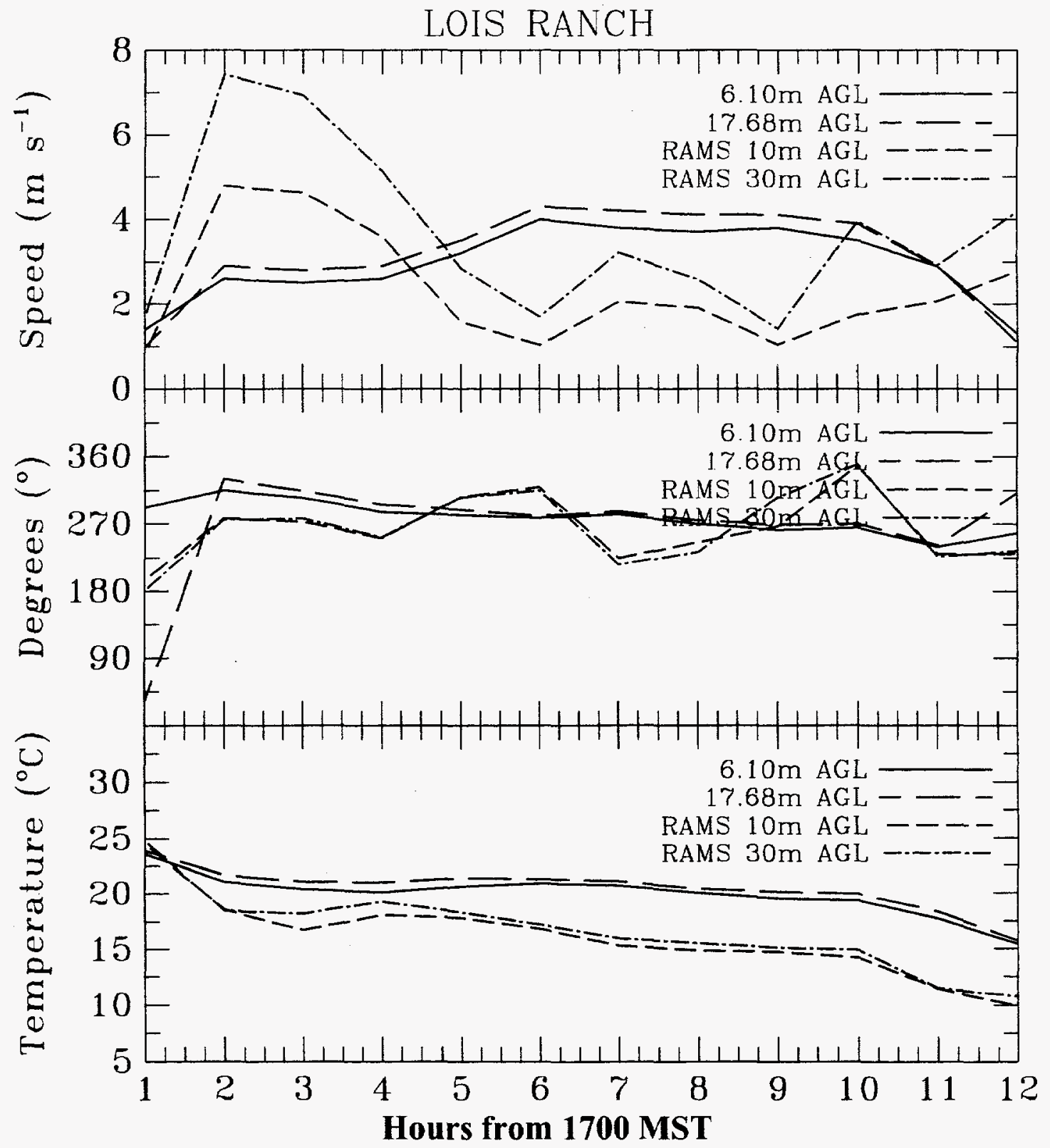

Figure 6.14 Observed and modeled 1-hour average a) wind speed, b) wind direction and c) temperature at the Lois Ranch ASCOT observational site for the overnight period 3-4 Sep 1993. The model heights do not correspond to the observational heights. 
underestimated wind speed during mid-simulation if mixing from aloft is important. The Pat's Flat site (Figure 6.15) comparison exhibits somewhat different features than the Lois' Ranch site. In particular, the wind speeds are captured more correctly, and the temperature prediction is closer In the early hours of the simulation wind speed is evolving correctly until hour 3 where the modeled wind speed reduces to less than observed values.

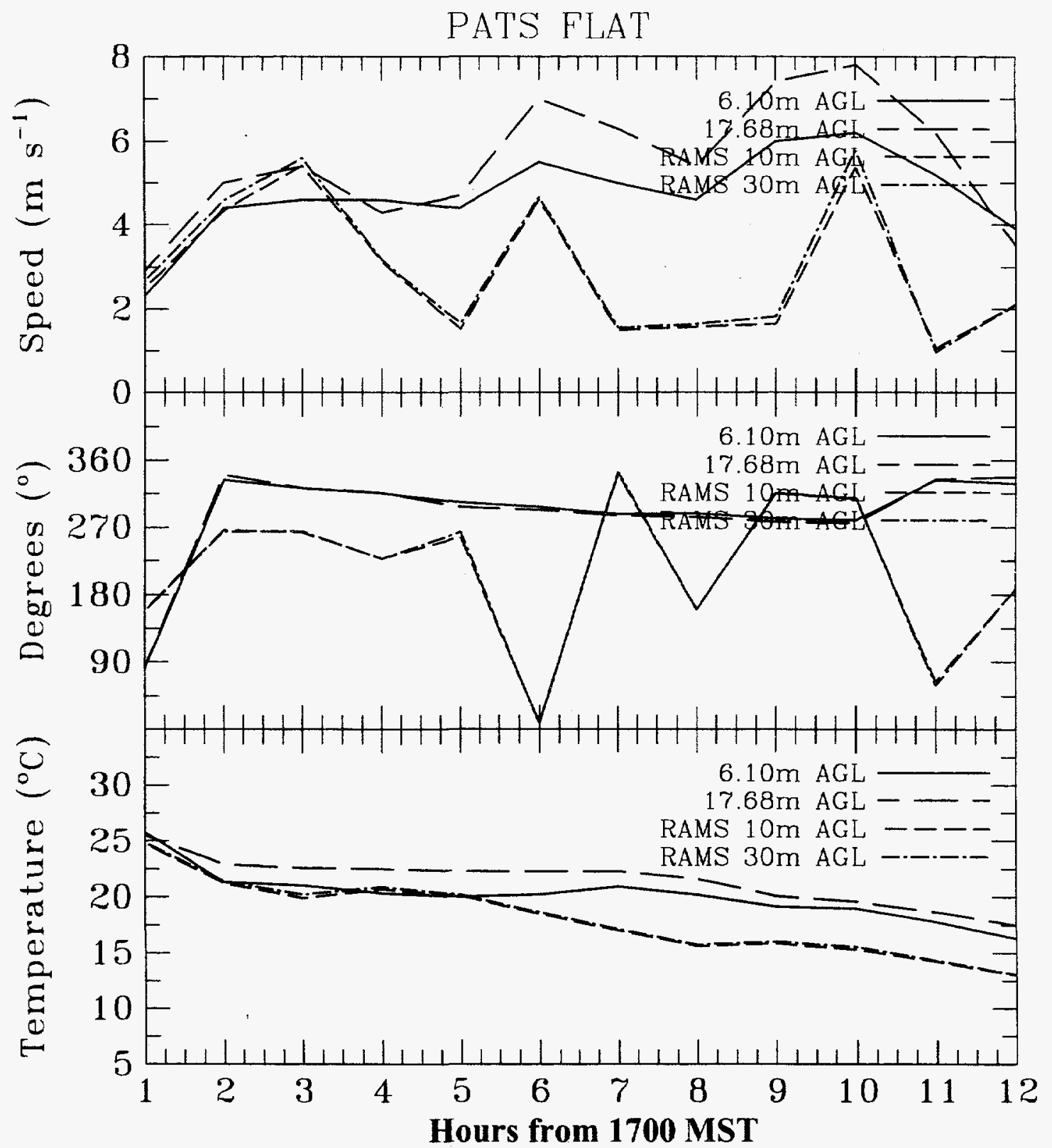

Figure 6.15 As in Figure 6.14 but for the Pats Flat ASCOT observational site. 
However, the two peaks in observed wind speed at hour 6 and hour 10 are partially captured by the simulation. Both of these peaks are associated with northerly flow, although generally the modeled wind direction contains too much westerly component. The temperatures agree well in the early hours but deviates, again too low in the latter 6 hours of the simulation. On a discouraging note, the modeled temperature difference between $30 \mathrm{~m}$ and $10 \mathrm{~m}$ AGL is very small, indicating less stable conditions than actually observed. This

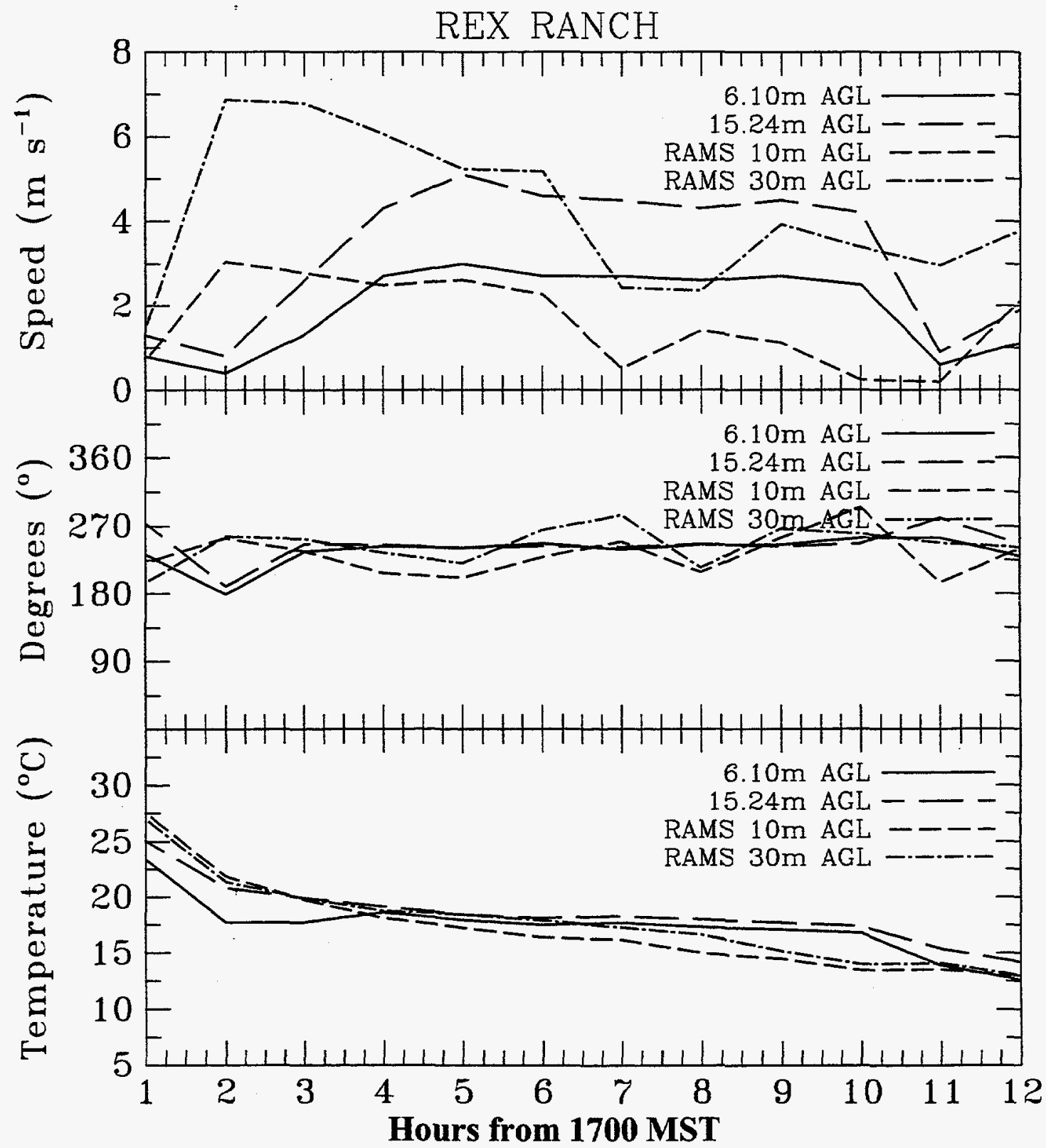

Figure 6.16 As in Figure 6.14 but for the Rex Ranch ASCOT observational site. 
correlates well with the significantly greater wind direction and speed variability at this site.

The modeled behavior at the Rex Ranch site can be described as good (see Figure 6.16), relative to the other sites because some significant features are simulated well. The wind speed plot indicates that throughout the night, the $15.24 \mathrm{~m} \mathrm{AGL}$ level wind was stronger than the $6.10 \mathrm{~m} \mathrm{AGL}$ level. While, the model solution does not capture the actual speeds exactly $\left(\sim 2 \mathrm{~m} \mathrm{~s}^{-1}\right.$ high or low after model spin-up), it consistently predicts a speed shear. Wind direction is correctly predicted to be about $245^{\circ}$, with somewhat greater variability. The modeled temperature is quite close to observations and trends downward. In this case an inversion condition is created between the $30 \mathrm{~m}$ and $10 \mathrm{~m}$ model levels.

\section{Summary}

The model prognosis of actual atmospheric conditions for the 3-4 Sep 1996 case night is reasonably good, based on a detailed comparison with observations. Large-scale aspects were well predicted, although this is to be expected due to the use of boundary nudging on the outermost grid points of Grid 1. Mesoscale features were captured sufficiently on the innermost grids; mountain wave and katabatic flows, in particular. In addition to inadequacies in the initial condition and the necessary use of parameterization, poorly predicted conditions could be explained by the imperfect terrain representation in the model and subsequent misrepresentation of terrain forcing at certain locations. Overall, the model performance is sufficiently good to give confidence that the majority of the relevant physical and dynamic mechanisms have been captured and that analysis of those mechanisms will be valid. That analysis is pursued in the following sections as well as in Chapter 7 , primarily by extracting additional model data. 


\subsection{KATABATIC FLOW ONLY (KFO)}

In addition to the realistic case, another simulation with no initial ambient flow or nudging but with radiative forcing active was completed. In this case, katabatic flows are expected to develop in earnest all over the domain. This simulation was run with the same configuration as the realistic case, except that the initialization was horizontally homogeneous. Thermal stratification was initialized to the actual potential temperature profile (as extracted from the Grand Junction sounding) for the case night, but winds were initially zero. The simulation began at $01 Z 4$ September, one hour later than the realistic case or MWO, to ensure that radiative cooling took effect immediately. The results from this simulation therefore represent the katabatic flows that would have developed on this case night had all winds suddenly become nil near the surface at sunset.

\subsubsection{General characteristics}

The quality of this simulation was generally good, with one main exception described in the next paragraph. There were no problems evident at grid boundaries or at the lateral boundaries of the grid. The complex terrain of the region was handled well by the terrain following coordinate in nearly all cases. The exceptions occurred at certain points on the innermost grid where topography exceeded $\sim 20^{\circ}$. At such points occasional $2 \Delta x$ noise was generated, but such noise is inherently damped by the turbulent parameterization and did not effect the simulation adversely. The steepest terrain resolved was to the north of Eldorado Canyon on the east side of the foothills and within the canyon itself. In these regions (i.e. Figure 6.17 at $X=5.0 \mathrm{~km}, Y=1.0 \mathrm{~km}$ ), the slope was $\sim 25^{\circ}$ and topography rose $9-10$ $\Delta \mathrm{z}$ across one horizontal grid point, whereas $14.04^{\circ}$ is the steepest topography allowed by the rule-of-thumb discussed in Section 4.3.1. In fact, there are 147 locations on Grid 5 that 


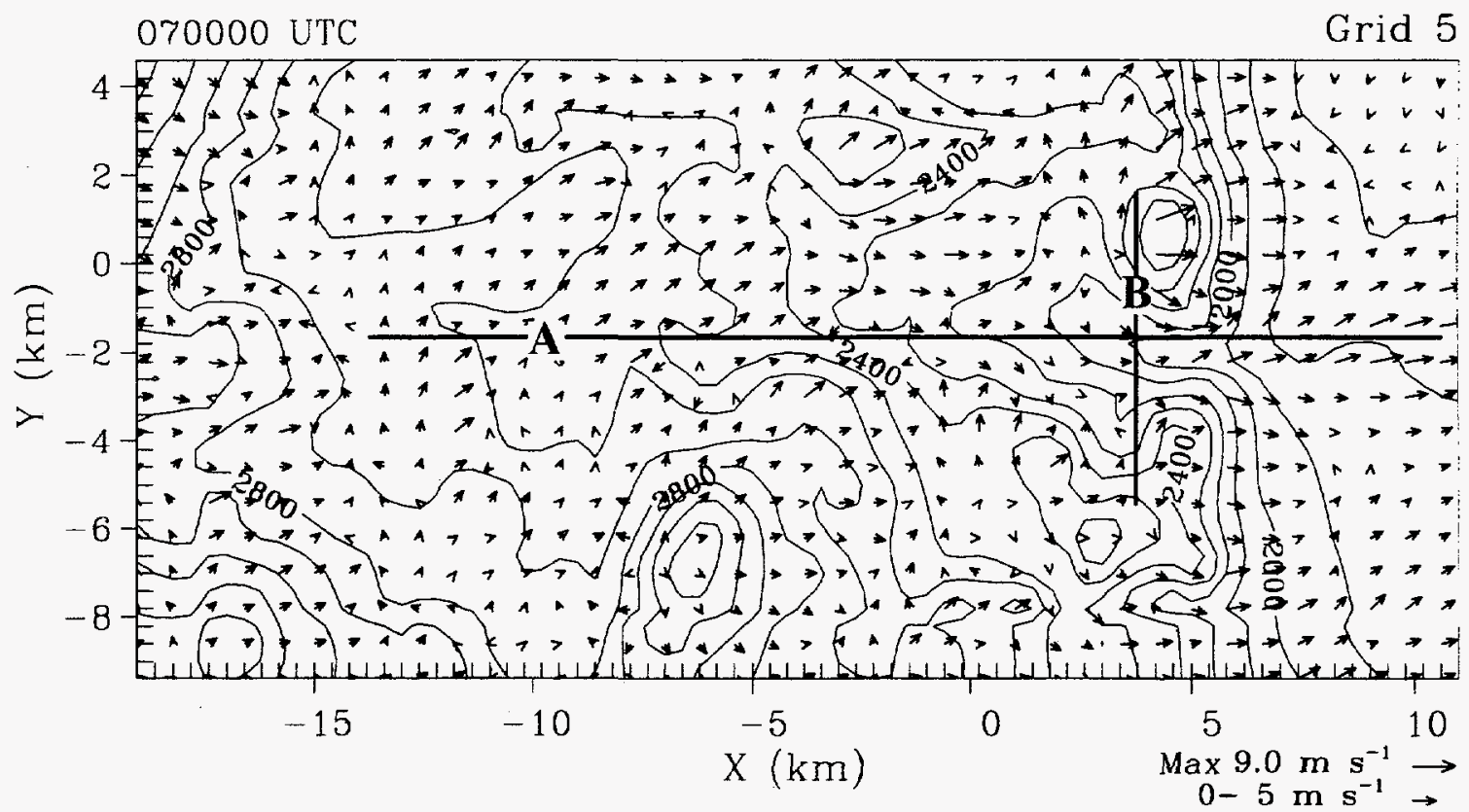

Figure 6.17 Horizontal wind vectors and topography ( $100 \mathrm{~m}$ contours) for $50 \mathrm{~m}$ AGL at midnight of the 3-4 Sep 1993 case night for an idealized, no wind initialization. (katabatic flow only) All flows are katabatic. The line labeled ' $A$ ' and ' $B$ ' indicate the location of the cross sections in Figure 6.19 and Figure 6.20, respectively.

violate the criteria (513 violations over all 5 grids), so the relative success of this simula-

tion and the other realistic topography runs is further proof that the rule-of-thumb is

unnecessary when resolution is sufficient to capture the phenomena of interest.

It should be noted, however, that after approximately 6 hours of simulated time, unrealistic cooling began to develop at certain grid points on Grid 5. Some of these problem areas were in the middle of the east-west extent of Eldorado Canyon, and thus the cold air was advected over other down canyon and plains locations by 8 hours of simulated time.

For the most part the following discussion (and that in Chapter 7) focuses on the first 6 hours of the katabatic flow only simulation, but where later times are mentioned the reader should treat the information with some caution if it involves the overcooled regions. This problem did not arise in either the mountain wave only or realistic simulations, and it is unclear exactly where the model problem originates from. Preliminary analysis has narrowed the error down to an interaction between the turbulent diffusion and surface layer 
parameterizations that causes very high cooling rates (over $50 \mathrm{~K}$ of cooling in 12 hours). Using the high time resolution output capability that the author added to the model code, timestep to timestep oscillations in sensible heat flux, friction velocity, $T^{*}$ and other surface layer quantities were found. These oscillations grow in time but are predominantly negative until they become so large that model execution stops. The turbulent parameterization becomes a factor because the surface layer calculation is applied at model level 2 in the midst of the turbulence parameterization just before scalar diffusion is begun. A test with flat topography did not contain the overcooling error, but the answer is not simply related to steep topography errors because the rapid cooling does not start at the steepest topography points. The most likely scenario is that oscillations, for some unknown reason, begin in the surface layer parameterization, whose overall effect is to cause rapid cooling at a particular grid point. This, in turn, creates strong horizontal gradients that are amplified in the turbulent parameterization by nature of the gradient errors in terrain-following coordinates in regions of high gradient. The root cause of the error is most likely in the surface layer parameterization, but, because of the strong interaction between surface layer calculations and the turbulent parameterization, it is difficult to ascertain the true cause. The problem can be solved if the source of the surface layer parameterization errors can be found, but efforts thus far, even under intense scrutiny, have been unsuccessful. It should also be noted that soil temperatures remain stable even while this error is rapidly growing and that the vegetation parameterization was not active in the tests.

As in the idealized simulations katabatic wind speeds achieved their maximums by $\sim 5$ hours after initialization, but their evolution thereafter was more variable. Figure 6.17 shows the horizontal flow field at midnight ( 6 hours after initialization) for model level 3 
or $50 \mathrm{~m}$ AGL. The winds mostly follow terrain contours as expected for katabatic flow and are relatively light compared to the winds just above level 3 . The maximum wind is evident just east of the exit of Eldorado Canyon. Outer grids (not shown) are also dominated by katabatic flow. Coal Creek Canyon also contains katabatic flow, but it is weaker and shallower than that in Eldorado Canyon, as typically observed. The slower speeds are most likely due to the smaller airshed and depth of Coal Creek Canyon, but may also be partly the result of somewhat worse topographical representation of Coal Creek Canyon by the given data set and chosen grid spacing.

The strong flow over the mountain just north of Eldorado Canyon is an interesting feature, because katabatic flows would not normally be expected to be high at a local topographic maximum. An analysis of the model fields shows that this feature is the result of strong westsouthwest katabatic flow generated in the upper, southwest-northeast oriented portion of Eldorado Canyon impacting the mountain barrier. As the strong katabatic upper canyon flow approaches the clockwise turn in Eldorado Canyon (at $X=5 \mathrm{~km}$ in Figure 6.17) where the topographic gradient becomes east-west oriented, its momentum causes it to continue eastnortheastward despite the changing topography. This phenomena is analogous to when the driver of an automobile approaches a curve with enough velocity that the automobile exceeds the road boundaries, (in effect tracing an arc of radius greater than that defined by the curve). Just as the next likely occurrence for the driver unable to correct his overshoot is to impact an obstacle outside the road boundaries, the katabatic flow remains north of the curve in the topographic gradient long enough to impact the mountain just north of Eldorado Canyon.

This phenomena is illustrated in Figure 6.18 which contains 3 cross sections through the mountain feature; wind vectors overlaying potential temperature, cross canyon winds and 


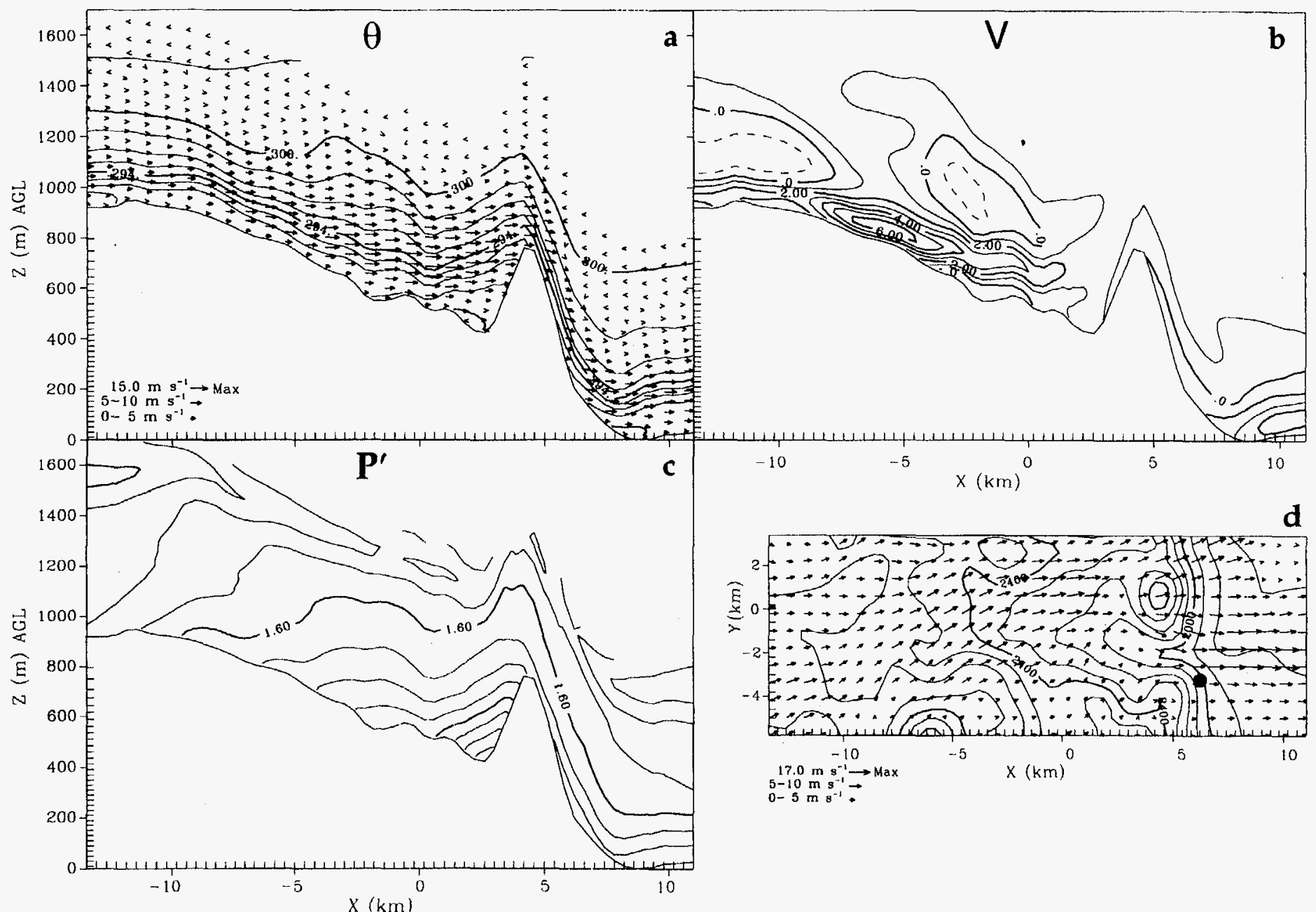

Figure 6.18 Plots of the variables that show the cause of the wind maximum at the peak of the mountain just to the north of Eldorado Canyon at midnight of the KFO simulation (see dot in panel d). a) - c) are east-west cross sections through the peak of a) wind vectors and potential temperature in $1.5 \mathrm{~K}$ intervals, b) cross canyon (or north-south) component winds in $1.0 \mathrm{~m} \mathrm{~s}^{-1}$ intervals and c) perturbation pressure in $0.20 \mathrm{mb}$ intervals. $\mathrm{d}$ ) is a close up of the region showing wind vectors at $110 \mathrm{~m}$ AGL. Note that vectors in d) follow the topographic contours faithfully in the upper canyon, but less so in the mid-lower canyon. 
pressure perturbation, and a $110 \mathrm{~m} \mathrm{AGL}$, terrain-following plan view of wind vectors. The local terrain minima at the westward mountain base is caused by a north-south oriented tributary to Eldorado Canyon. Unlike Poulos and Bossert (1995), where this tributary drained into the main canyon with significant southerly katabatic flows, this simulation shows domination by overrunning katabatic flows from the west. At the lowest levels, where the westerly flow must diverge around the obstacle, the tributary flow is in an opposite sense to the expected katabatic flow direction (see Figure 6.17 and 6.18b).

From Figure $6.18 \mathrm{~d}$ one can see that the strong westerly component shown approaching the mountain is slowed at $110 \mathrm{~m}$ AGL before accelerating as it surmounts the obstacle. The vertical cross sections in Figure $6.18 \mathrm{a}$ and $\mathrm{b}$ clearly show that strong west-southwesterly flow enters the region about $10 \mathrm{~km}$ west of the obstacle $(X=-6.5 \mathrm{~km})$. This flow has katabatic jet structure in both its $\mathrm{u}$ and $\mathrm{v}$ components. The perturbation pressure field in Figure $6.18 \mathrm{c}$ shows that the pressure gradient force at the westward base of the mountain is directed westward and upward, causing both deceleration and lifting of the incoming flow. As the flow rises over the mountain it accelerates slightly, maintaining a jet structure as it descends the steep $\left(25^{\circ}\right)$ eastern foothills. Noting the location of the $294 \mathrm{~K}$ contour at mountain top relative to the $300 \mathrm{~K}$ contour to the east over the plains, this rapid descent is caused by strong negative buoyant forcing. As the broad, deep katabatic flow from the upper canyon impacts the obstacle, some of its momentum is forced into the northern part of Eldorado Canyon, whose flow is discussed further below.

\subsubsection{Lower Eldorado Canyon katabatic flow}

A vertical cross section of wind vectors overlaid with potential temperature (Figure 6.19a)reveals considerable detail about the lower Eldorado Canyon flow. This section is 


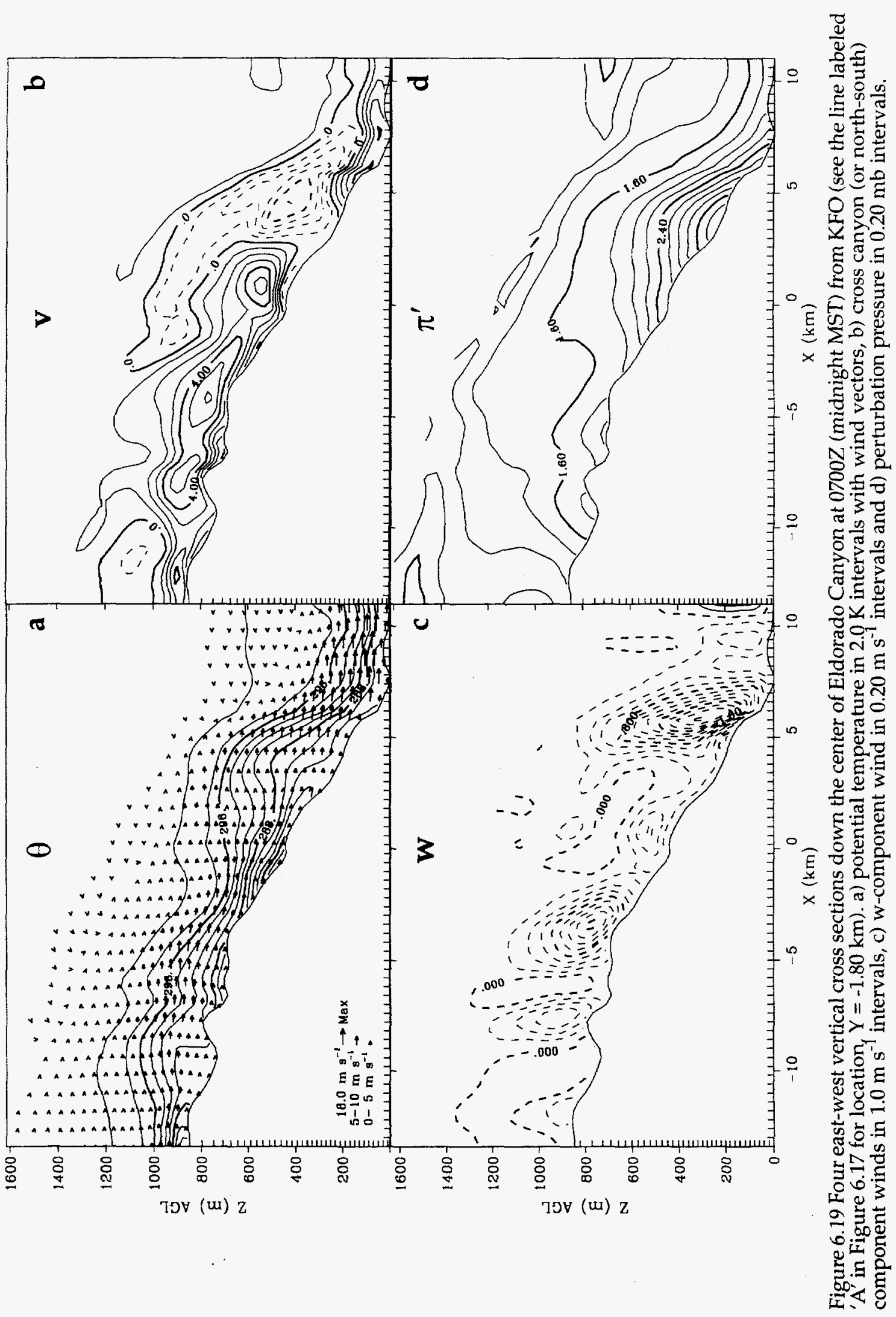


taken at midnight along an east-west line through the mouth of Eldorado Canyon (see line ' $A$ ' in Figure 6.17). Strong stratification has developed in the canyon, with the exception of the lower $200 \mathrm{~m}$ from $\mathrm{X}=2.5-5.5 \mathrm{~km}$, and katabatic flows enter $(X=-3 \mathrm{~km})$ and exit $(X=5 \mathrm{~km})$ the lower portion of the canyon near $15 \mathrm{~m} \mathrm{~s}^{-1}$. While seemingly high based on experience, the idealized simulations indicate that such speeds will form in the model in undisturbed radiatively cooled conditions. If one further considers the converging of several individual slope and tributary flows into this canyon, such speeds are reasonable. Katabatic flow near this magnitude was occasionally observed during the ASCOT campaign; at times, tethersondes at the exit of Eldorado Canyon could not remain flying due to excessive wind speeds and shear out of the canyon mouth. The Eldorado Canyon katabatic flows are the largest in the entire domain at any level.

An interesting high pressure, low wind speed feature develops at $X=3-4 \mathrm{~km}$ in the lower levels as seen in Figure 6.19a and d. There is also a noticeable upward bulge in the potential temperature field and nearly vertical isentropes near the surface at this location. Up and down canyon of this feature, isentropes generally slope downhill (a smaller, similar bulge exists at $X=-10 \mathrm{~km}$ ). To the east of the $X=4 \mathrm{~km}$ point significant katabatic flow jets reappear in earnest as the canyon converges into a more narrow form. Near the surface upstream of the bulge, a pool of air 4-6 K colder than surrounding surface air has formed. What is the likely cause of this upward bulge and what is its significance?

An interesting series of dynamical mechanisms combine to form these features. It is clear from $6.19 \mathrm{~d}$ that a bulge of perturbation pressure coincides with the deviation of isentropes from the surface. A local maximum in $p^{\prime}$ of $3.40 \mathrm{mb}$ is located at $\mathrm{X}=3.5 \mathrm{~km}$ so that the pressure gradient force is oppositely directed away from this area on either side of 
the high pressure. The upstream pressure gradient force ( $p^{\prime}$ contours that slope down and to the west) decelerates the westerly flow from $5-10 \mathrm{~m} \mathrm{~s}^{-1}$ to near calm for $100-200 \mathrm{~m}$ AGL. This calm region, without significant shear in the vertical, allows the development of the cold pool above the high pressure region. If calm winds are conducive to the development of this cold pool, why doesn't the cold pool extend further down canyon into the high pressure region? This is explained by the north-south vertical cross sections through the local high in Figure 6.20. Of particular importance is panel $c$, where a local maximum in TKE is evident at the same location as the local high. An along canyon section of TKE (not shown) verifies that this TKE maximum is localized in the east-west direction as well. As a result, locally stronger mixing occurs and cold air pooling is unable to develop.

So then, what causes the development of the local high if conditions are well-mixed and the typical mechanism of cooling induced pressure increase is inoperative at the surface? First and foremost, the air above the local high has, in fact, cooled significantly. Noting the north-south section of potential temperature in Figure 6.20a, the column of air over the high has reduced significantly from its initial temperature near $300 \mathrm{~K}$. This cooling has occurred throughout the domain however, so another mechanism must be at work. Panel $b$ of Figure 6.20 indicates that strong v-component convergence exists over the canyon above this point. Since the perturbation component of the non-hydrostatic Exner function (or pressure) must increase where convergence occurs, a local high develops (u component divergence was negligible). The terrain and wind field configuration shown in Figure 6.18d shows that local flows converge as 1) katabatic flows from opposing slopes or tributaries meet, and 2) westerly flows diverge around the mountain to the north of Eldorado Canyon (see previous section). The overall effect is the development of a local high and 


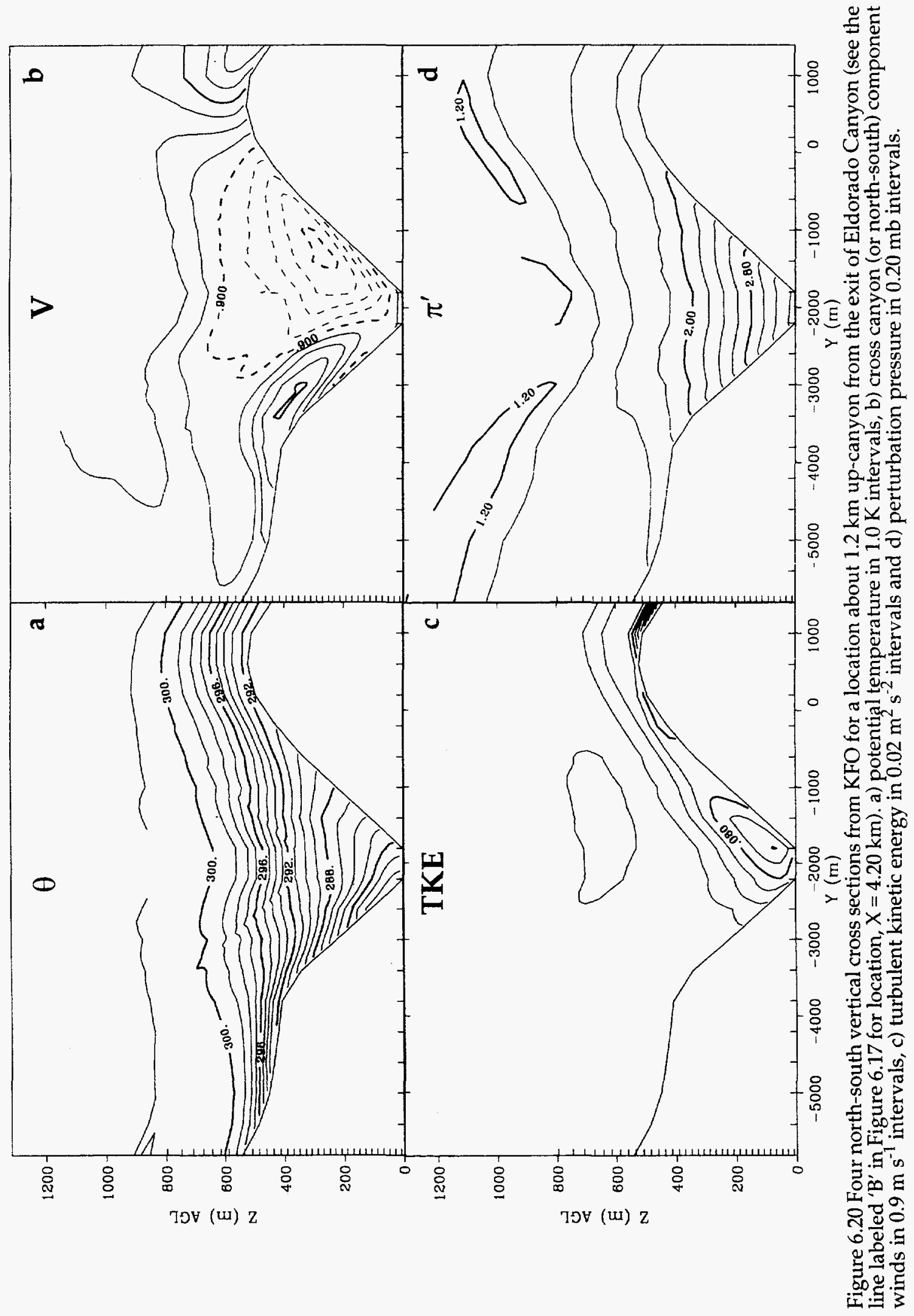


subsequent upward and up canyon pressure gradients, preventing the continued downward progress and acceleration of katabatic flow. In turn, cold air flows over the local high (bulging) before accelerating and descending rapidly on the east side of the feature (a 2 $\mathrm{mb}$ perturbation pressure gradient exists across $7 \mathrm{~km}$ from the local high to the plains!).

The weakened downward vertical motions, and even some upward vertical motion, are shown in Figure 6.19c for the bulged region. Upward or weak vertical motions also coincide with smaller potential temperature bulges up canyon.

\subsubsection{Eldorado Canyon air parcel dispersion}

We have not yet come to understand the trajectory an air parcel would take if released into this katabatic flow environment. By using a Lagrangian Particle Dispersion Model some of the conclusions about flow structure (and trajectories) above can be verified. For this experiment, 5 particle sources were placed in various Eldorado Canyon area locations throughout the Grid 5 model domain (see Figure 6.2e). The LPDM was started at 7 hours into the model simulation (0100 MST) and run for 5 hours using wind information from the RAMS model simulation in 0.5 hours increments. Particle sources were defined as 30 $\mathrm{m}^{3}$ cubes centered on a point $45 \mathrm{~m} \mathrm{AGL}$ at a given horizontal location and are referred to by number. The turbulent parameterization was activated to more realistically portray perturbations to air parcel trajectories. Additional simulations with various source heights below $100 \mathrm{~m} \mathrm{AGL}$, including one with source heights at $2.5 \mathrm{~m} \mathrm{AGL}$ and $5 \mathrm{~m}^{3}$ release volumes, led to the same conclusions as presented below.

The LPDM result is summarized by the plan view of particle position 3 hours after LPDM start in Figure 6.21. As described earlier in the meteorological analysis, particles from the upper canyon, sources 1 and 5, are carried in katabatic flows whose destination is 


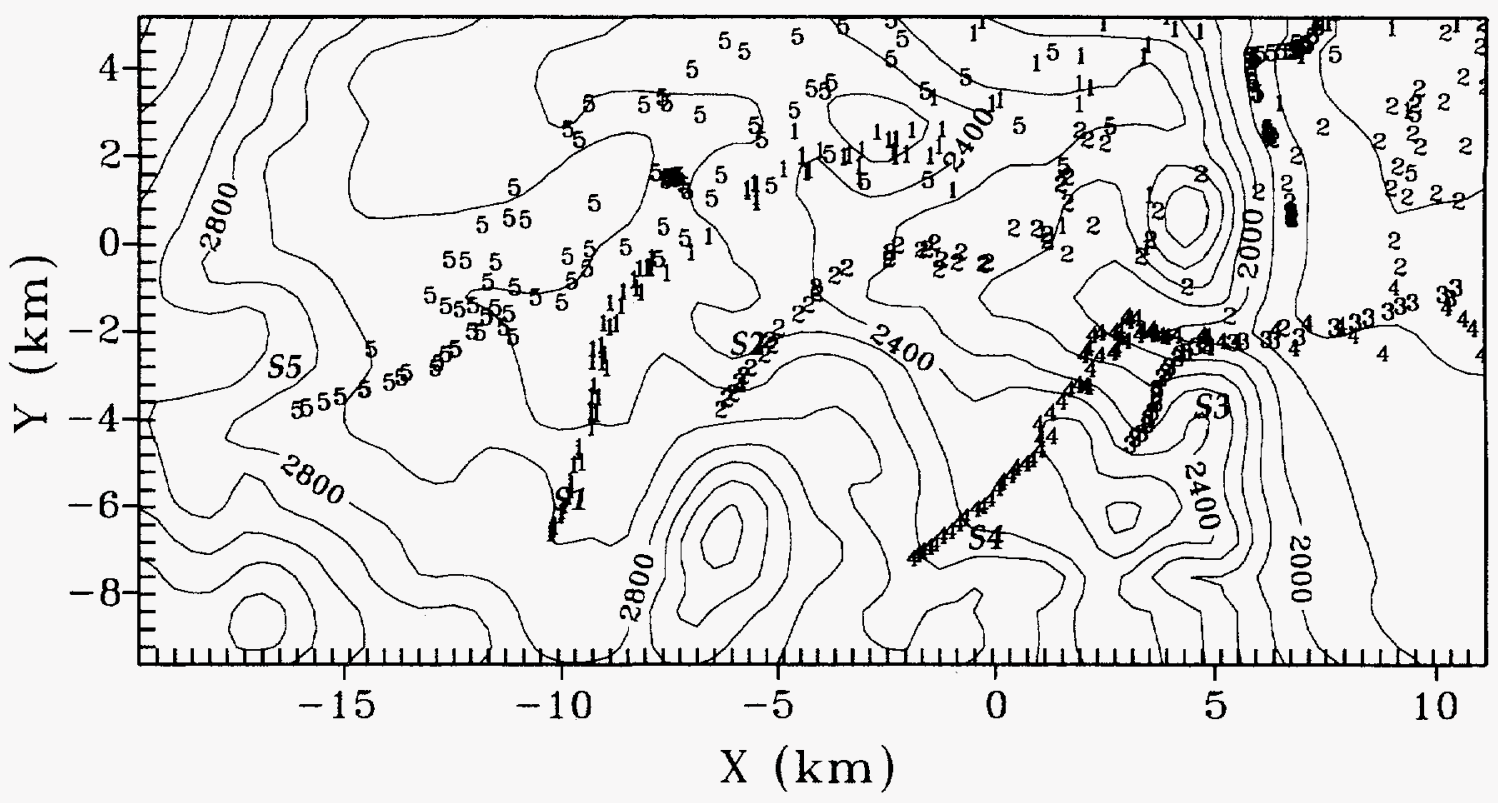

Figure 6.21 Particle trajectories from 5 sources within Eldorado Canyon at 3 hours into a LPDM simulation of the katabatic flow only case. The LPDM was started at model time + 7hrs. Particle release locations are labeled S1 - S5.

not the mouth of Eldorado Canyon. Instead, these particles are carried across the lowest canyon walls (mid-canyon) and into the next canyon north. This result has serious implications for how the term 'airshed' for a canyon should be defined. Unlike water in a watershed, air in an airshed is not necessarily true to its topographic confinements - even in the most undisturbed conditions represented here. In fact, it would appear that katabatic flows from approximately the upper $50 \%$ of the canyon are not confined by the canyon walls.

Unlike sources 1 and 5, the particles released from sources 3 and 4 are confined to Eldorado Canyon only. A steady train of particles from each source travel primarily down the topographic gradient to the northeast and drop into the lower, east-west oriented portion of the canyon. After a $60^{\circ}$ clockwise turn the particles accelerate to the east and onto the plains. This behavior is most easily understood and intuitive. In this portion of the canyon topographic trapping is complete for the simulated flow speeds.

The particles from source 2 take various routes to the plains from their initial position on the south wall approximately $11 \mathrm{~km}$ west of the canyon mouth. At various times during 
the dispersion simulation, the trajectories of source 2 particles vary from a path north of Eldorado Canyon to one that tracks toward the mountain just north of the mouth of Eldorado Canyon. The latter of these two trajectories is shown in Figure 6.21 and particles have three route possibilities once encountering the barrier, around to the north, over the peak or around to the south. Regardless of the route taken, each particle, just as with the other 4 sources, eventually reaches the plains.

\subsubsection{Katabatic only simulation summary}

The katabatic flow only simulation gives us important insights about the dynamics of complex terrain katabatic flows, while also showing the likely maximum katabatic flow scenario for the Eldorado Canyon region for this case night. Upper canyon katabatic flows are not confined to the defined canyon boundaries. The strong flow in Eldorado Canyon is generated primarily by converging katabatic flows in the lower, more confined canyon section. These flows combine to create some unusual pressure-temperature profiles as the canyon narrows. It should be clear that more interesting details could be discussed with regard to this simulation, but the previous discussion forms a basis for comparison with both the mountain wave only and realistic simulations (already discussed somewhat in Section 6.4). That is, because this investigation focuses on influences on katabatic flows by overlying mountain waves, it is important to understand the fundamental katabatic flows that would develop over the region of interest if undisturbed. Discussion of the mountain wave only case follows.

\subsection{Mountain WAVE ONLY (MWo)}

The next logical step before discussing the interaction of mountain waves with the katabatic flows for this case night is to simulate the conditions that would form if no radi- 
ative forcing existed at all. This is the basis of simulation MWO, where all characteristics are the same as the realistic simulation except that the radiation parameterization is not active. We expect that surface cooling will be nearly eliminated, aside from that which is nudged in and/or advected from the nudging boundaries (recall that nudging of conditions was only on the outermost 5 grid points of Grid 1, an upstream distance of $\sim 500 \mathrm{~km}$ ). As described earlier, the overnight meteorological condition was $6-8 \mathrm{~m} \mathrm{~s}^{-1}$ flow from $320^{\circ}$ due to the southwest-northeast oriented synoptic pressure gradient and clear skies. The Froude number averaged near 0.45 .

The upper level features of the mountain wave only simulation were quite similar to those of the realistic simulation already described in Section 6.4. In particular, a lee trough developed more strongly at $700 \mathrm{mb}$ than at $500 \mathrm{mb}$ (not shown), of similar amplitude and location as that in the realistic case (see Figures 6.4 and 6.5 ). The flow upstream of the Continental Divide and Grids 4 and 5 was similar to that of the realistic case at higher levels, but noticeably different in lower levels (below $\sim 500 \mathrm{~m} \mathrm{AGL)}$ ) The differences that are significant to the interaction and evolution of mountain wave and katabatic flow will be discussed in greater detail in the next Chapter.

Figure 6.22 shows a series of vertical cross sections on a line through Eldorado Canyon across the Continental Divide on Grid 4 in three hour increments. The mountain wave structure varies in time as flow of varying strength encounters the Rocky Mountain barrier. At 3 hours of model integration (2000 MST, Figure 6.22a) a nearly neutral boundary layer is advecting into the Grid 4 domain at lower levels. Above this layer more thermally stratified air exists and mountain wave structure is well defined. Individual isentropes, such as the $318 \mathrm{~K}$ contour, descend approximately $900 \mathrm{~m}$ or 0.45 of the Front Range relief 


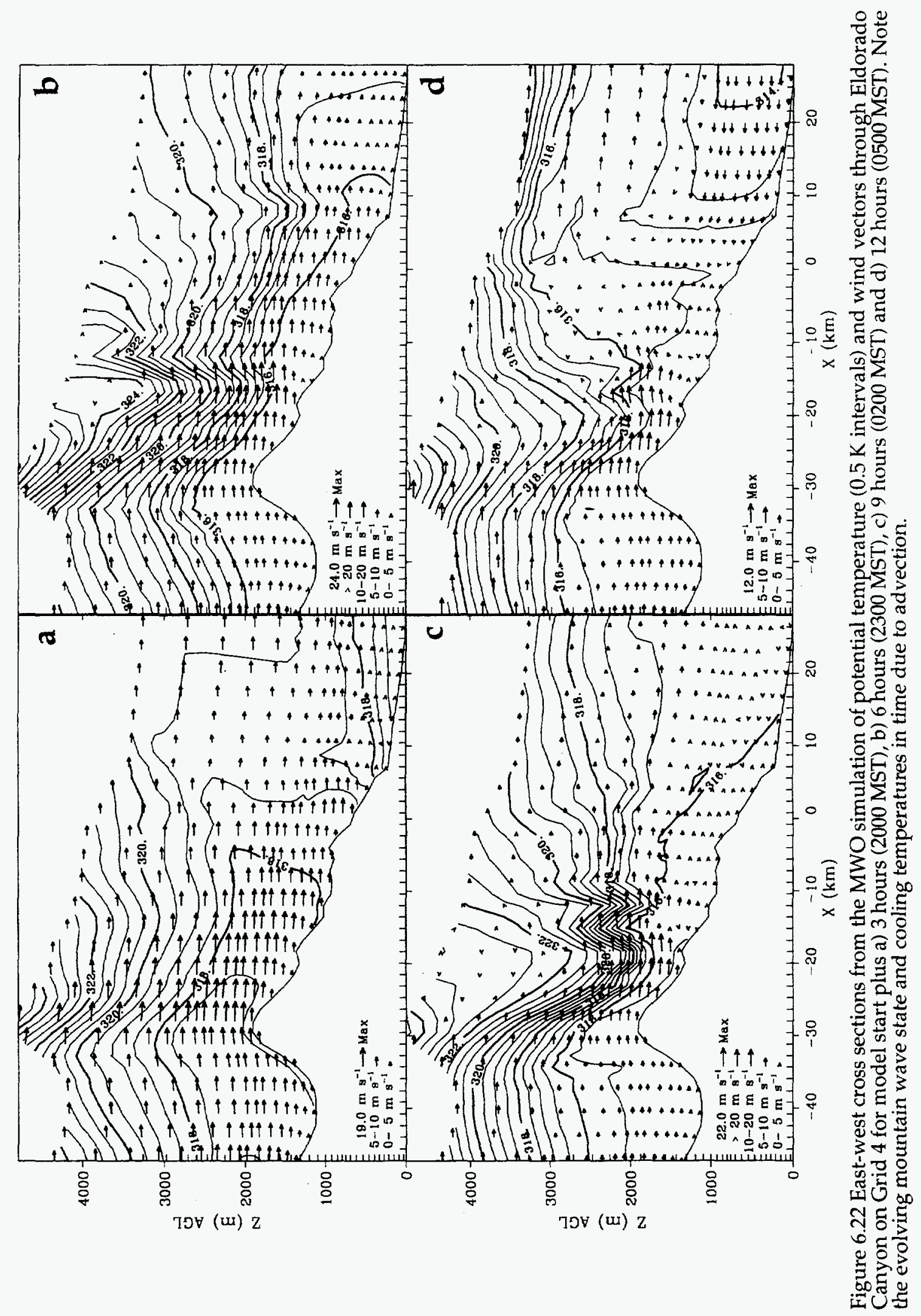


from their peak elevation, which is consistent with the upstream average Froude number of 0.45 . By 2300 MST potentially cooler air has advected into the lower domain and stronger stratification aloft causes wave amplification and wavelength reduction. As the wave again rises at $\mathrm{X}=-15 \mathrm{~km}$, strong shear and a weak rotor form along the mountain side. Stronger flow descends to near the surface just east of the rotor. The flow on the plains remains weak. Mountain wave wavelength shortening continues at 0200 MST and wind maxima remain above $20 \mathrm{~m} \mathrm{~s}^{-1}$ on the east side of the Continental Divide despite weaker upstream flow. The wavelength is sufficiently contracted and upper air flow sufficiently stable that the strongest momentum in the mountain wave remains aloft east of $X=10 \mathrm{~km}$. Weak flow exists below $1 \mathrm{~km}$ east of this point and, if under the influence of radiative cooling, one might expect katabatic flow development to occur despite the overlying mountain wave, as was actually found in the realistic simulation. At 0500 MST (Figure $6.22 \mathrm{~d}$ ), the stratification aloft has again relaxed and the mountain wavelength is broadened. Once again the strongest mountain wave flow impact the near surface region just east of the Continental Divide. An easterly flow has developed over the plains in a neutral layer that may be shear induced. Upward vertical motion and divergence aloft at $X=0 \mathrm{~km}$ indicate that a circulation has developed where the easterly plains flow and westerly mountain wave flow meet. A similar cross section for Eldorado Canyon on Grid 5 (not shown) replicates the descriptions above. Additional information about MWO is given in the following Chapter.

\subsection{SUMMARY}

Three topography simulations have been described in the preceding sections. These simulations were 1) realistic, where the best possible forecast of case study conditions was 
made, 2) katabatic flow only (KFO), where the MAPS nudging of the MWO case was deactivated and replaced with horizontally homogeneous, no wind initial conditions, and 3) mountain wave only (MWO) where the radiation parameterization was not used, although all other aspects of the realistic case were retained. The realistic case study simulation produced atmospheric fields that compared reasonably well to the observations taken by the ASCOT experimental program for the 3-4 Sep 1993 case night. Observations from wind profilers, sodars and towers were used in the comparisons. Generally, the experimental data was not sufficient to extract information on the interaction of katabatic flow and mountain waves, but the model results are good enough to act as a surrogate data set. The katabatic flow only simulation developed katabatic flows up to $20 \mathrm{~m} \mathrm{~s}^{-1}$ in Eldorado Canyon, although not all katabatic flows that developed initially within the traditionally defined airshed remained within the airshed. Because there were no external influences on katabatic flows in KFO, and yet katabatic flows left the airshed confines, the definition of the term 'airshed' must itself be questioned. The mountain wave only simulation. forced by 6 hourly MAPS analysis data sets, showed a highly evolutionary state. The mountain wave amplitude was found to be very sensitive to changes in atmospheric thermal stability and mountain wave momentum at various times could either strongly or weakly influence near surface flows. 


\section{CHAPTER 7}

\section{ThE INTERACTION AND EVOLUTION OF THE Phenomena}

\subsection{OVERVIEW}

In previous Chapters we have developed a comprehensive understanding of katabatic flow and the state of knowledge concerning its interaction with mountain waves/ambient flow (Chapter 2), the conditions of the case night in question (Chapters 3 and 6), the behaviour of simplified simulations of this interaction (Chapter 5) and in Chapter 6, of the three topography simulations. The topography simulations have yet to be analyzed completely with regards to their contribution to the understanding of MKI, however. The main goal of this chapter is to extract this contribution. The approach taken is to analyze a number of different measures of MKI, not unlike what was done in Chapter 5. After first examining absolute differences between the simulations, vertical integrals and alterations to the dynamics of the flow due to the overlying mountain wave via model forcings, will be discussed. To give further order to the analysis, three locations in the domain were chosen for discussion, 1) an upper Eldorado Canyon location close to the Continental Divide, 2) a lower Eldorado Canyon location, and 3) a plains location. These locations can be identified as points 30,19 an 8, respectively, in Figure 6.2e. Still greater simplification was made by choosing 2 vertical levels of the 50 available (except in the case of vertical inte- 
grals) at each of these three points. From Grid 5, model level 3 at $50 \mathrm{~m}$ AGL was selected to represent a near surface point likely to be under the influence of katabatic forcing, and model level 13 at $250 \mathrm{~m}$ AGL was chosen as a vertical elevation where either katabatic forcing or mountain wave dynamics might dominate, depending on their relative strength.

\subsection{SiMULATION DIFFERENCES}

Some insights into MKI can be garnered by looking at differences in meteorological variables as predicted by model. In the following, differences among the three realistic topography simulations described in the previous chapter for 4 model variables, the $u, v$ and $\mathrm{w}$ wind components and potential temperature are examined.

\subsubsection{Wind differences}

The non-linear nature of the interaction of katabatic flow and mountain waves is shown for the $u, v$ and $w$ components of the wind in Figures 7.1-7.3, respectively. Values are for three different horizontal points within the Grid 5 domain, the upper canyon, the lower canyon and east of the canyon mouth on the plains. On these plots are the 3rd model vertical level above ground, plotted with thick lines, and the 13th model vertical level, plotted with thin lines., The 3rd and 13th model levels correspond to $50.0 \mathrm{~m}$ and $250.0 \mathrm{~m}$ AGL, respectively, for $\mathrm{u}$ and $\mathrm{v}$ and $60.0 \mathrm{~m}$ and $260.0 \mathrm{~m} \mathrm{AGL}$, respectively, for $\mathrm{w}$ because of the staggered grid structure (C grid, Winninghoff 1968, Arakawa and Lamb 1977) used in the numerical model.

\section{Upper canyon}

The upper canyon u component information (Figure 7.1a) shows that the realistic simulation (solid lines) tended toward the mountain wave only solution at both upper and lower levels. In the first five hours of the simulations the realistic case had the strongest upper 
level, upper canyon winds, due to intensified stratification from radiative cooling compared to the MWO simulation. While both the realistic and MWO simulations trend downward in $u$ after 9 hours, the realistic simulation does not have the severe flow reduction of the mountain wave only simulation at 9.5 hours. It is interesting to note that whereas upper level $u$ exceeds that of lower levels in Figure 7.1a, the same cannot be said for $\mathrm{v}$ (Figure 7.1a) where strong low-level southerlies underlie weak upper level (thin

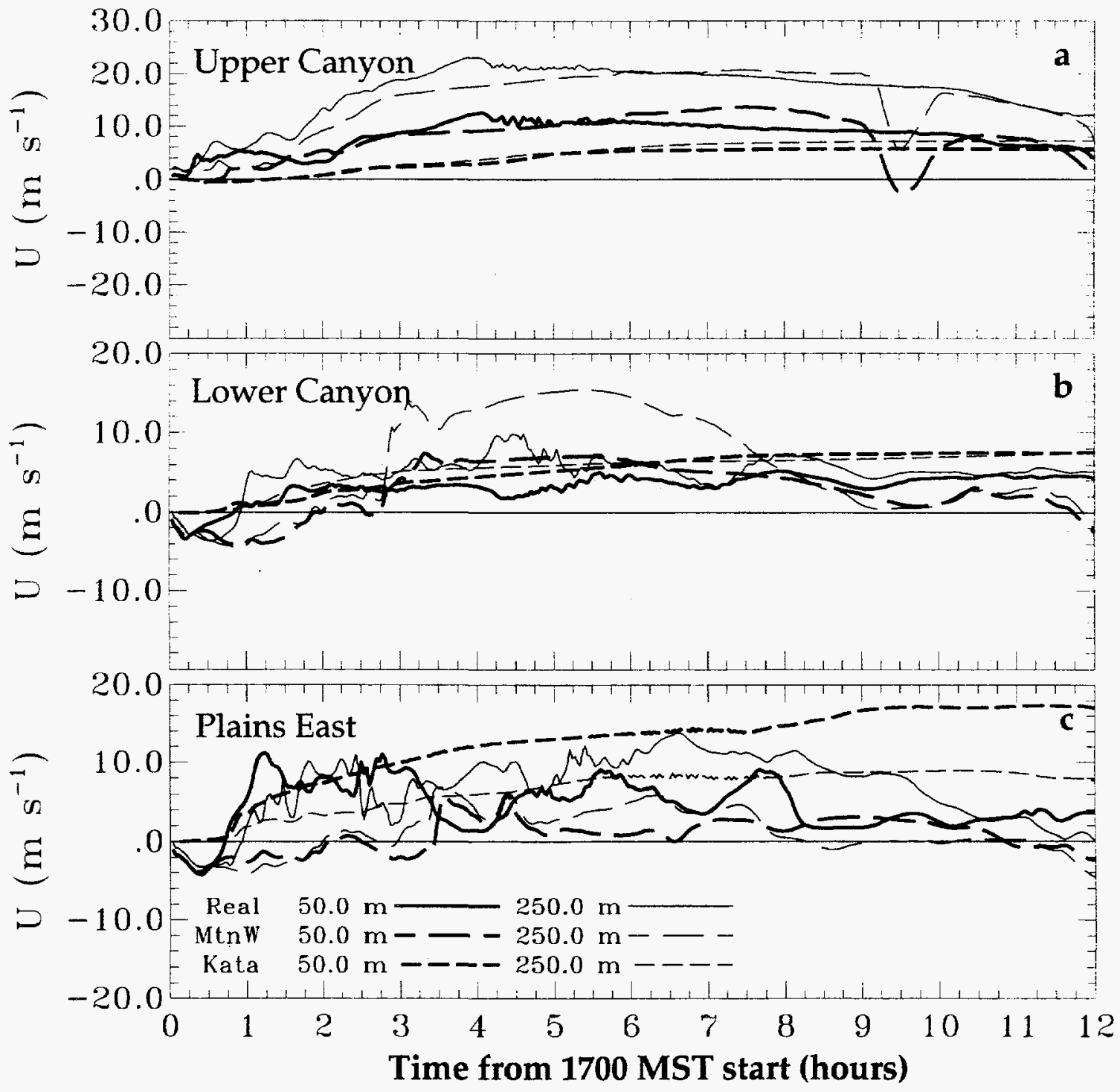

Figure 7.1 Two-minute data interval, east-west component winds versus time at three different horizontal locations on Grid 5 , a) an upper canyon location at grid point coordinate $(5,13)$ or $X=-17.6 \mathrm{~km}, Y=-4.8 \mathrm{~km}, \mathrm{~b})$ lower canyon at $(60,20)$ or $X=4.4 \mathrm{~km}, Y=-2.0 \mathrm{~km}$, and c) on the plains $\sim 2.5 \mathrm{~km}$ east of Eldorado Canyon at $(72,23)$ or $X=9.2 \mathrm{~km}, Y=-0.8 \mathrm{~km}$. Results are shown for the realistic (solid lines), KFO (long dashes) and MWO (short dashes) simulations at $50 \mathrm{~m}$ (thick lines) and $250 \mathrm{~m}$ (thin lines) AGL. 
lines) northerlies. This effect can be explained in the upper levels of the realistic and mountain wave simulations by the ambient northwesterly flow overflowing the Continental Divide as a mountain wave (see point \#30 in Figure 6.2e). At lower levels, the southerlies are created by channeling of the ambient flow by the small north-south tributary this upper canyon point lies within. Note that the v-component at upper levels of the realistic simulation between 2.0 and 3.75 hours is northerly (during the time westerly flow is accelerating; Figure 7.1a), indicative of scouring by the northwesterlies. After 3.75 hours the vcomponent turns southerly, more like the mountain wave solution where pressure-driven effects such as described by Whiteman and Doran (1993), are dominant. After 4.25 hours, the $v$ component of the realistic solution becomes noisy for an hour as the solution oscillates nonlinearly between the KFO and MWO solutions. This variability corresponds with the increased amplitude of the mountain wave and subsequent wave breaking aloft. Upper level wave breaking seems to be the cause of gustiness below and has a strong influence on near surface flow fields. At $50 \mathrm{~m} \mathrm{AGL}$, the realistic solution v component has similar features as the KFO solution, except in the first 2 hours, and also has gustiness at hours 45. It is most interesting that neither of the other two realistic topography simulations (KFO or MWO) experiences this gusty period. The fact that the v-component of the realistic simulation exceeds that of the katabatic flow simulation at low levels indicates that katabatic flow is enhanced by pressure-driven channeling (Whiteman and Doran 1993). A comparison of model forcings on the v-component at this level (not shown) indicates that advection is a positive forcing on $\mathrm{v}$ whereas in the katabatic flow only simulation advection is a negative effect. At this point in the upper canyon the interaction of mountain waves and katabatic flow occurs in a variety of ways. 


\section{Lower canyon}

The lower canyon horizontal position can be found on Figure 6.2e where the plot is labeled '19'. This point is deep within the lower canyon and we expect it to be dominated by katabatic flow for two main reasons, 1) in the deeper lower canyon, cooling, and therefore the pressure gradient, is enhanced by its greater topographic amplification factor (McKee and O'Neal 1989, Whiteman 1990), and 2) the lower canyon is the most topographically sheltered location. Looking at panel $b$ of each of Figures $7.1-7.3$, it is immediately evident that overall, horizontal velocity is lower than in the upper canyon, implying a

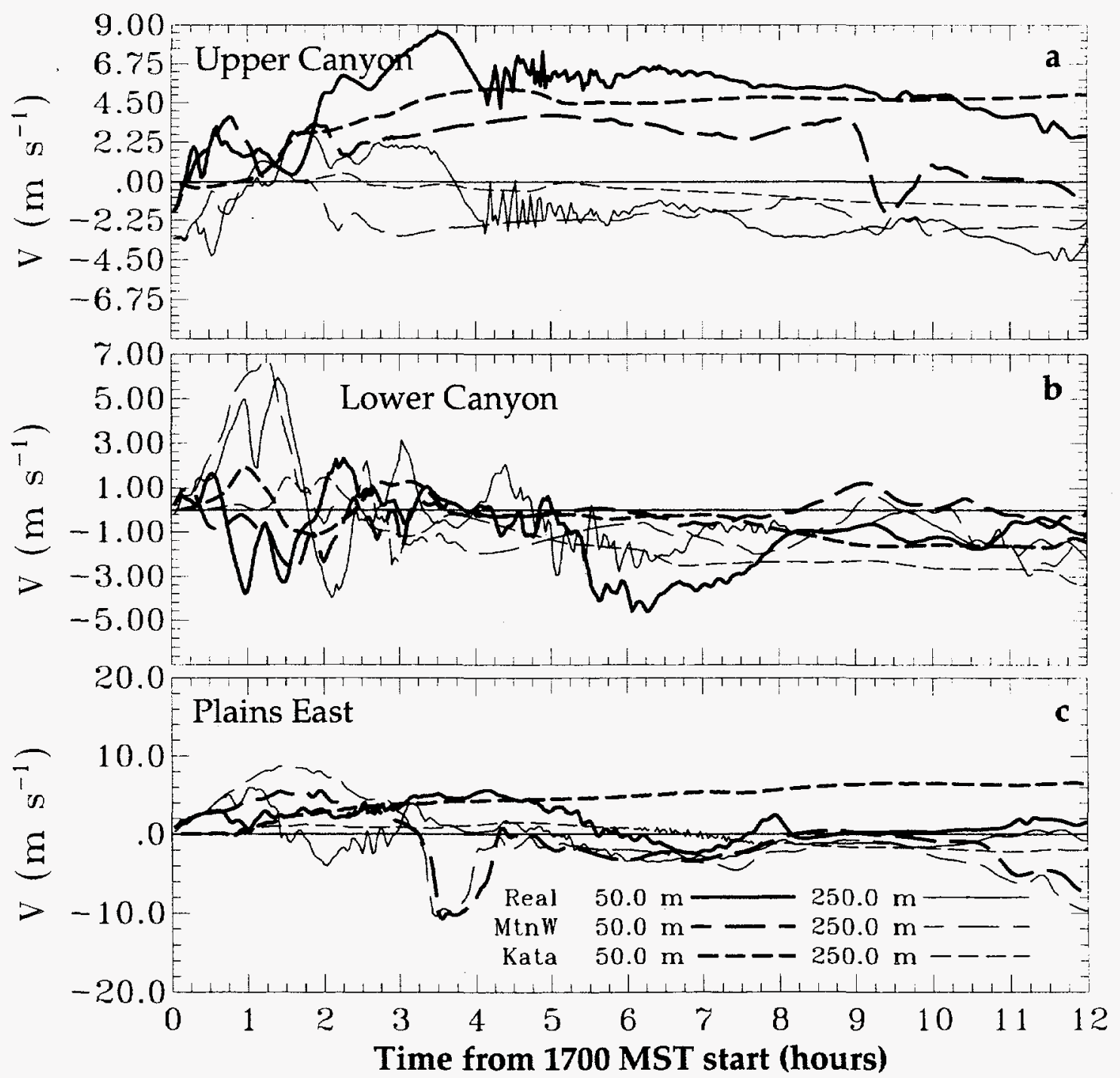

Figure 7.2 The same as Figure 7.1 but for v-component. 
degree of mechanical sheltering from mountain wave momentum. This is partly because the grid point representing lower canyon conditions is at a point where a convergenceinduced local high pressure forms, reducing katabatic flow at this spot. This mechanism has been discussed in Section 6.5. Just east of this point, flows accelerate to over $15 \mathrm{~m} \mathrm{~s}^{-1}$ in the realistic and $\mathrm{KFO}$ simulations as canyon topography converges. In the first 6 hours of the simulations, $u$ is reasonably similar among them, except that in the early stages the realistic and KFO runs have a negative u-component due to initialization. The other exception is at $250 \mathrm{~m}$ AGL of the MWO simulation, where from 3.5-8.0 hours strong flow exists aloft. This is caused by the relatively long wavelength of its mountain wave due to the lack of radiative cooling, compared with the realistic simulation. Eventually, however, the wavelength of $\mathrm{KFO}$ wave is reduced as flow changes induced by the nudging influence the inner domain. Thus, after 8 hours in MWO, the surface point where the mountain wave lifts off the surface is located up canyon from this point, reducing the $u$ wind speed. For the latter 10 hours of $\mathrm{KFO}, \mathrm{v}$ is very small, primarily due to topographic sheltering. The mountain wave strength during the strong $u$ wind period of MWO can also be verified by the strength of $w$ in Figure $7.3 \mathrm{~b}$ where downward vertical motion experiences a local maximum.

In the realistic simulation, lower canyon winds generally remain small due to the protective effect of stratification in addition to the shortening of mountain flow wavelength. For this reason, lower canyon winds track primarily with the katabatic flow only simulation, implying that katabatic forcing dominates mountain wave forcing at this deep canyon location. The $\mathrm{v}$ component wind does, however, experience a local low level maximum between 5.0 and 7.0 hours in the realistic simulation. This is caused by surface based 


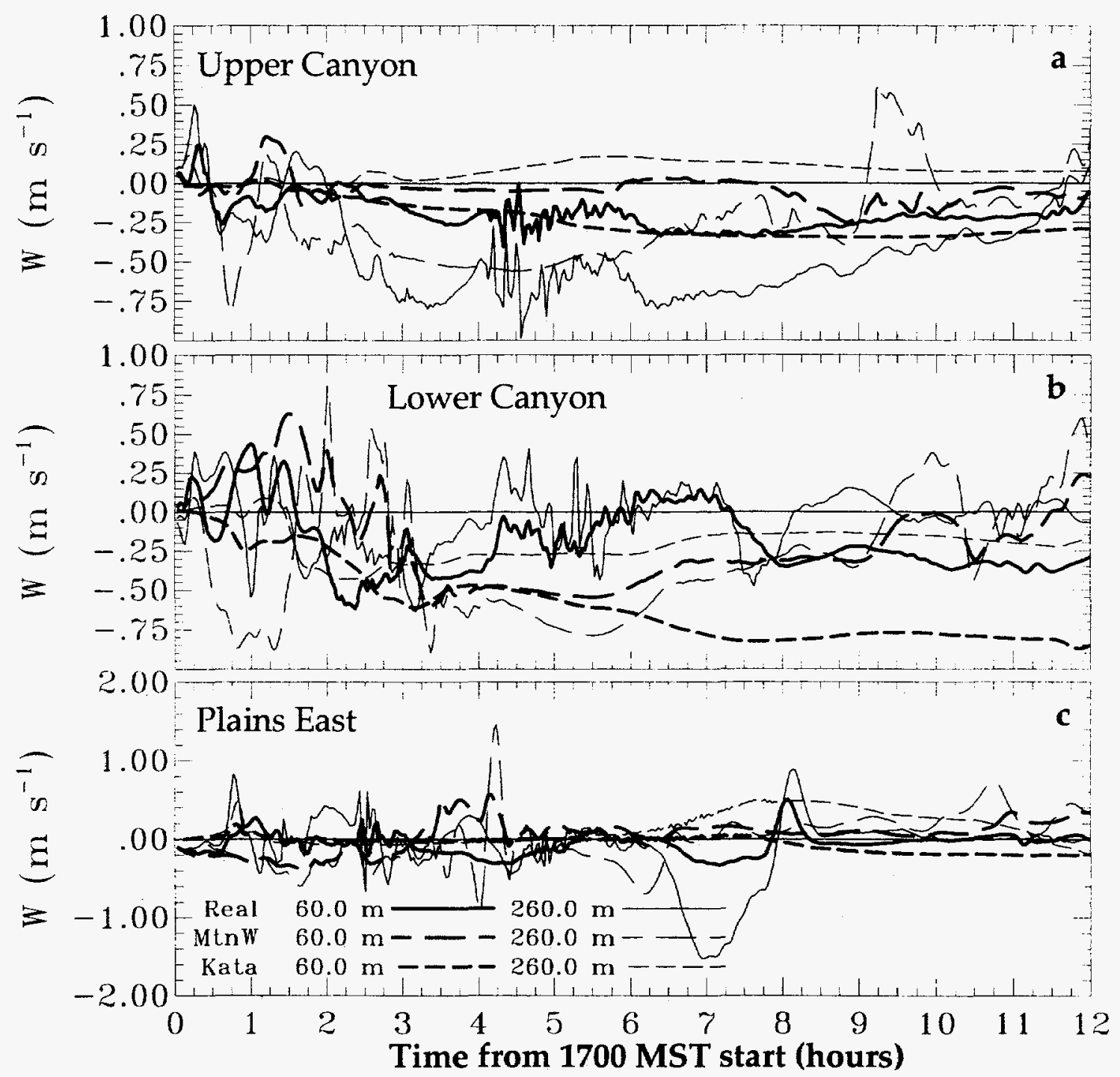

Figure 7.3 The same as Figure 7.1 but for w-component.

katabatic flows from the lower canyon's northern tributary, that are somewhat eroded by the overlying flow. In the katabatic flow only simulation, where erosion from aloft does not occur, katabatic flow is deeper after 5 hours of simulated time.

\section{Plains East}

From 1.0 to 11.0 hours of simulated time the katabatic flow on the plains, as represented by point $\# 8$ on Grid 5 (see Figure 6.2e), has jet structure and is from the southwest. At 6.0 hours, for instance, the $\mathrm{u}$-component wind speed at 50 and $250 \mathrm{~m}$ is $13.0 \mathrm{~m} \mathrm{~s}^{-1}$ and $6.0 \mathrm{~m}$ $\mathrm{s}^{-1}$ (Figure 7.1c), respectively, and the v-component is $4.0 \mathrm{~m} \mathrm{~s}^{-1}$ and $1.0 \mathrm{~m} \mathrm{~s}^{-1}$ (Figure 
7.2c), respectively. Clearly, a strong low-level katabatic jet exists. The reason the katabatic flow turns to the north after exiting Eldorado Canyon, particularly at low levels, is that the topographic gradient of South Boulder Creek on the plains is southwest-northeast. Note that from 6.0 to 8.0 hours the katabatic flow at this point changes from a west-southwest orientation to west and then to west-northwest. This transition is accompanied by some instability and coincides with a transition to upward vertical motion, particularly at upper levels. This transition is caused by very cold air exiting Eldorado Canyon at 6.0 hours (see Figure 7.4c) and an increase in inversion strength, such that katabatic flow from the foothills west of this point, which is potentially warmer than the Eldorado Canyon low level flow, overrides the strong inversion without being channelled to the northeast.

By comparison, the flow over the plains in the realistic simulation is far more variable, as should be expected due to the overlying mountain wave. This increased variability, alone, represents the interaction of a mountain wave with developing katabatic flow. Due to greater mixing, the katabatic flow jet is less well-defined, located at a higher elevation and, at times, flow is dominated by mountain wave momentum. The greater mixing also prevents the development of very low near surface temperatures in the realistic, thereby reducing the katabatic forcing mechanism.

\subsubsection{Potential Temperature differences}

A plot of the potential temperature evolution is presented in Figure 7.4. In the upper canyon the $50 \mathrm{~m}$ AGL of the realistic simulation closely follows that of the KFO simulation after 2 hours. The same cannot be said for the $250 \mathrm{~m}$ AGL level, where $\theta$ is less than that of KFO. Thus, although lower level stratification prevents complete scouring by the mountain wave, cold air is better mixed to higher elevations. The mountain wave only 
simulation shows no inversion whatsoever as this region, due to a lack of radiative cooling, is easily scoured and mixed to a neutral state. For this reason the potential temperature lines for MWO significantly overlap each other.

In the lower canyon a vastly different behaviour is found for the realistic case at $50 \mathrm{~m}$ AGL compared to the KFO simulation. Whereas the total cooling at the $50 \mathrm{~m}$ height for both the realistic and katabatic flow only simulations in the upper canyon was about $10^{\circ} \mathrm{C}$, that in the lower canyon section was $15^{\circ} \mathrm{C}$ in the realistic case, but an unrealistically large

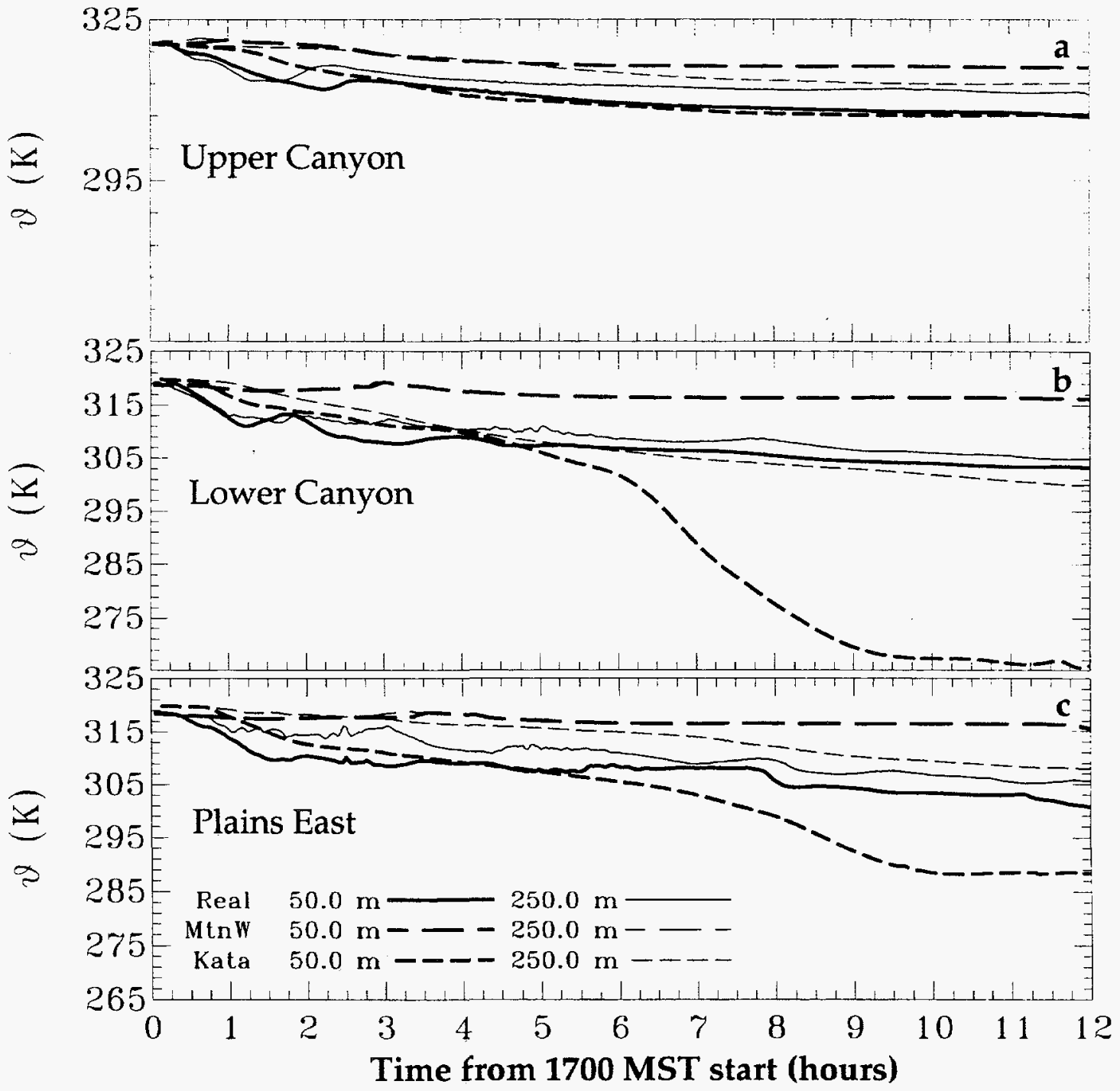

Figure 7.4 The same as Figure 7.1 but for potential temperature. The lines for the mountain wave only simulation are nearly always overlapped and indistinguishable. 
$55^{\circ} \mathrm{C}(!)$ in the katabatic flow only simulation. This is by far the most unrealistic feature of any of the simulations and it is caused by strong near surface radiative cooling and a lack of mixing at this location. This model problem is discussed in Section 6.5 on page 189 . and is limited to the lower half of Eldorado Canyon only for the latter 6 hours of the simulation. It is clear that the local high pressure that develops over this part of the lower canyon, thereby reducing wind speed, helps induce this behaviour. A long standing problem with the turbulence and surface layer parameterizations in the stable boundary layer obviously needs to be addressed.

In most of the domain, however, the simulations proceeds normally, and the question of MKI in temperature distribution can be considered. On the plains, the temperature of the realistic simulation steadily cools - even more so than the katabatic flow only simulation at upper levels. This behaviour is due to additional mixing caused by the overlying mountain wave, and causes the katabatic flow jet to be weaker and more elevated than in the katabatic flow only case. Dynamically, this is understandable because the jet location appears where the sum of positive pressure gradient forcing due to cooling and negative frictional forcing is maximized. In the katabatic flow only case, this location is a lower elevation because conditions are less disturbed and strong stratification reduces turbulence more quickly with height. This argument is supported by the fact that 1) the katabatic flow jet of the realistic simulation is indeed more elevated than the katabatic only simulation, and 2) the inversion strength is weaker in the realistic case.

\subsection{VERTICAL INTEGRALS}

Vertical integrals can be used to summarize time evolution, vertical atmospheric structure, mass transport, circulation strength and model trends. This section presents vertical 
integrals from each of the three simulations for vertical and horizontal mass flux, and heating. Integrals of velocity fluxes are taken upward vertically, so that mass transport eastward (upward) is indicated by a positively sloped line for the $u(w)$ mass flux. For heating integrals the heat content at a particular model time is calculated relative to the heat content at the initial time, and heating is summed downward. In this case, cooling is indicated by positively sloped lines. In all cases, the closer the plotted line is to an infinite slope, the closer the mass flux or heating is to zero (i.e. no additional contribution to the integral) at that elevation AGL. Note, further, that for brevity, only integrals calculated for the lower canyon point will be shown, although the upper canyon point and plains point will be discussed where significant information arises relative to MKI. In most cases, however, integrals from the other sites do not add, but only support, the conclusions given here.

\section{Integrals of u-component flux}

The relative similarity of panels $b$ and $c$, and the relative dissimilarity of panel $a$ from

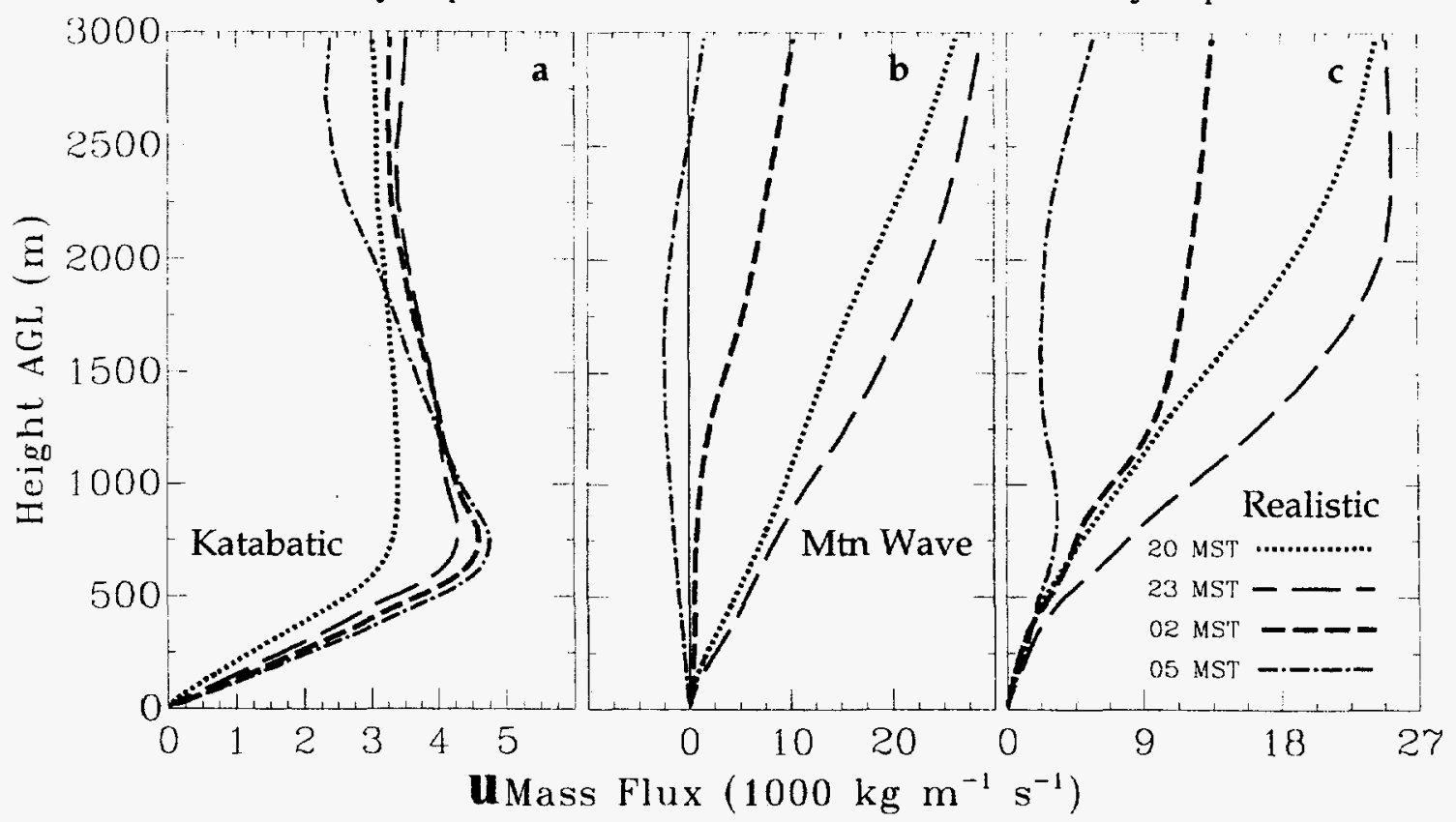

Figure 7.5 The upwardly summed vertical integrals of westerly mass flux $\left(1000 \mathrm{~kg} \mathrm{~m}^{-1} \mathrm{~s}^{-1}\right)$ for 2000 MST (short dashed curve), 2300 MST (long dashed curved), 0200 MST (medium length dashed curve) and 0500 MST (alternating short-long dashed curve) for simulations a) katabatic flow only, b) mountain wave only, and c) realistic. The integral was taken at point \#19 in Figure $6.2 \mathrm{e}$, also referred to as the 'lower canyon' point in Section 7.2. 
these, is the first, immediately apparent, quality of the u-component mass flux comparison in Figure 7.5. This difference is caused by the strong mountain wave flow in the realistic and mountain wave only simulations, and the lack thereof in the katabatic flow only simulation. Mass flux at the uppermost levels is more than 5 times stronger in panels $b$ and $c$, than in panel $a$, at 3 hours into the simulations (2000 MST). This difference diminishes toward the end of the simulations, however, as easterly momentum enters the Grid 5 domain. By 0500 MST in the mountain wave only simulation the integrated mass flux becomes negative, indicative of this gradual transition. In contrast, the integrated mass flux of the realistic simulation never reaches zero indicating a net eastward transport of mass at all times. While the sum of panels $a$ and $b$ (not shown) clearly does not equal the results of panel $c$, the addition of radiative cooling to the mountain wave only simulation obviously alters the mass balance of the Front Range flow. For most of the realistic simulation's length the positively sloped line indicates net westerly flux (occasionally near zero), with the most notable exception appearing at the end of the simulation (0500 MST). By comparison, the $\mathrm{u}$-component mass flux integral of the KFO simulation has a stronger negative slope aloft, indicative of the easterly return flow above the westerly katabatic current. Therefore, one of the modifications made to katabatic flow by an overlying mountain wave is to mask the return current. By $0500 \mathrm{MST}$ in the realistic simulation, however, a weak return flow does develop as weakening westerly momentum creates a more favorable environment for return flow to be revealed.

The profile of $u$ mass flux for the katabatic flow only simulation in Figure 7.5a, as with all but one of the other profiles, shows that there is not a closed circulation over this horizontal point. Stated another way, the westward mass flux in the return flow aloft is not 
large enough to compensate for the eastward mass flux by westerly component, low-level katabatic flow. Mass flux profiles to even greater heights indicate that this is indeed the case for the atmosphere above this point - the mass flux profile never returns to the zero line. This situation is similar at the plains location (not shown), and reveals that mass in this region will only balance over time if easterly flow develops during the daytime. Vertical integrals of u mass flux from Wolyn and McKee (1992) for the Front Range north of this location, indicate that flows with sufficient mass flux can be generated by daytime upslope flows. The return circulation of the katabatic flows generated in this simulation exceed barrier height ( $\sim 1700 \mathrm{~m}$ above this point). Although katabatic flow generated in the lower canyons and on the plains does not develop sufficient return flow to close the katabatic circulation, the katabatic flow at the upper canyon point is able to do so by later in the simulation. By speculation, it is possible that return flows at this elevation are enhanced as they are confined by thermal stratification while rising toward the Continental Divide.

\section{Integrals of w-component flux}

The evolving katabatic flow and mountain wave are easily seen in Figure 7.6 where vertical mass flux varies significantly between plotted times for each simulation. At 2000 MST in panel $a$ the katabatic flow simulation contains downward mass flux throughout this $3000 \mathrm{~m}$ depth indicating a local supply of air from above. Later, after the entire Front Range katabatic flow system has developed, the vertical mass flux is nearly constant above $\sim 1500 \mathrm{~m} \mathrm{AGL}$, showing that a much smaller proportion of air in the local vertical column is resupplying mass to the local katabatic flow system. Although not shown, the upper canyon vertical mass flux has a negative contribution throughout its depth, indicat- 
ing that eventually some portion of the return flow moves downward to replace exiting mass to the Front Range thermally forced flow system. The time lag in the development of this system shows the inherently local nature of katabatic flows, but also that they can join together into a coherent, if incomplete, circulation system. The consistent kink in the vertical profile of katabatic flow only vertical flux at $\sim 400 \mathrm{~m}$ AGL is suggestive of a local return flow at low levels, as compared with the upper level larger-scale return flow. This separation of scales is consistent with the existence of the smaller scale katabatic flow system in the canyon within the larger Continental Divide scale katabatic flow system.

The canyon-scale return flow is masked by overlying mountain wave flow in Figure 7.6c. Evidence of katabatic flow to approximately the same depth as in the katabatic flow only simulation $(\sim 250 \mathrm{~m})$, is seen at 2000 MST and 0500 MST but only weakly at 0200 MST. The katabatic forcing is strongest in Figure 7.6c when horizontal mass flux in the lowest $1500 \mathrm{~m}$ (see Figure $7.5 \mathrm{c}$ ) is weaker. This is not a strong correlation, but the highest low level mass flux in Figure 7.6c, at 2300 MST, does correspond to the weakest katabatic

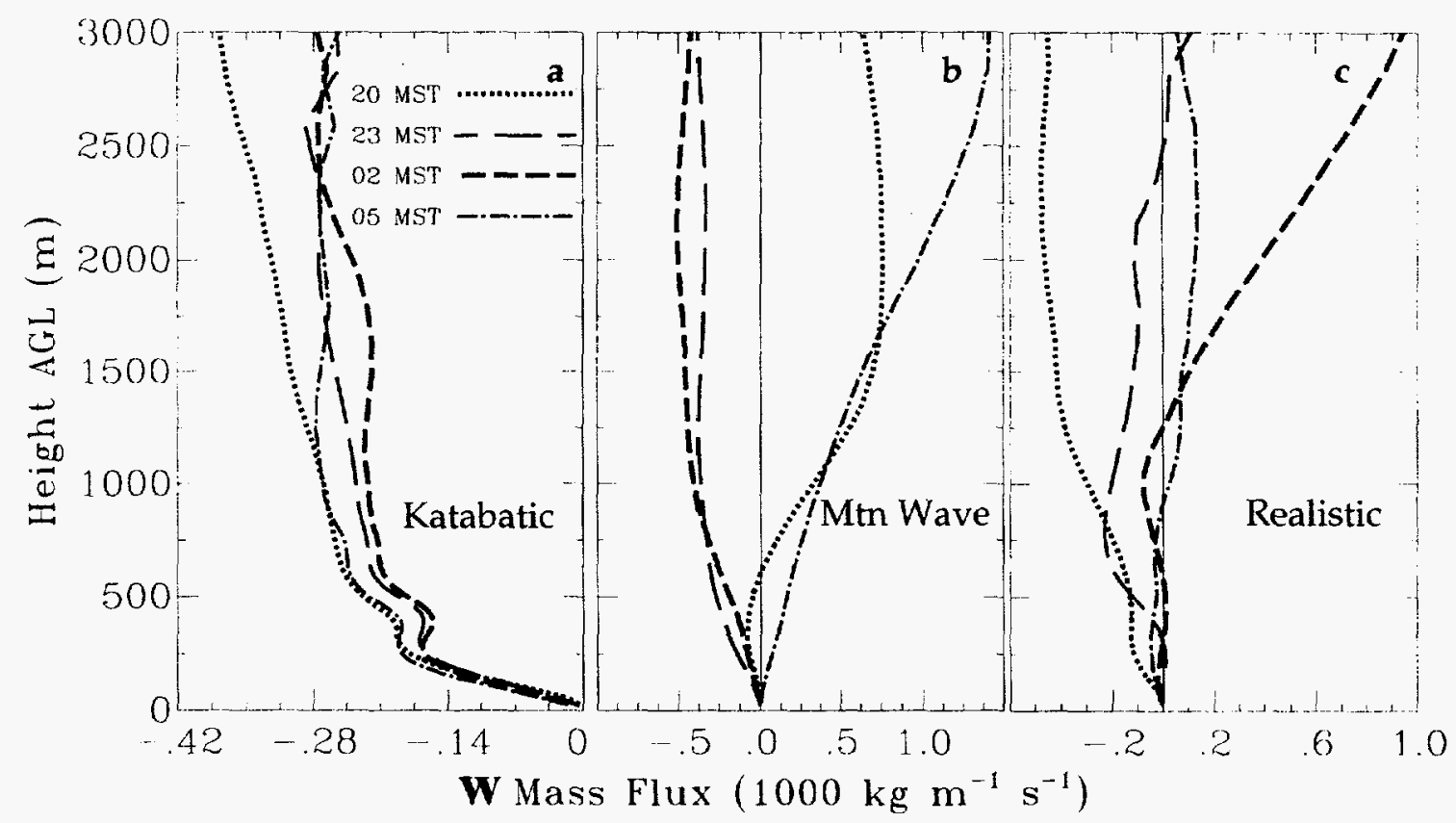

Figure 7.6 The same as Figure 7.5 but for vertical mass flux. 
flow condition at this point. In further support of this idea, a deep layer of downward vertical mass flux in the 2300 MST line of Figure 7.6c, indicates that this location was under the influence of relatively strong mountain wave dynamics at this time. An east-west plot of potential temperature and winds, verifies that the mountain wave over this location at this time not only contained the strongest winds of the overnight period $\left(\sim 25 \mathrm{~m} \mathrm{~s}^{-1}\right)$ but also had a large enough wavelength to impact this location rather directly.

The differences between Figures $7.6 \mathrm{~b}$ and $\mathrm{c}$, confirm that radiative cooling has a strong effect on mountain wave evolution overnight. Without radiative effects, the mountain wave vertical flux profile evolves from net upward flux, with little contribution above $1700 \mathrm{~m}$ AGL over this location (2300 MST), to net downward mass flux with little contribution above $1000 \mathrm{~m}$ AGL (2300 MST and $0200 \mathrm{MST}$ ), to net upward mass flux throughout the $3000 \mathrm{~m}$ integral by simulation end. By adding radiative effects to the MWO simulation in the realistic run, this evolution is completely changed. The mountain wave amplitude is modulated so strongly by developing overnight stratification that at 2000 MST the realistic simulation places the descending portion of the mountain wave over this location (net negative downward mass flux), where the mountain wave only simulation places the upward portion of the wave over this location. In the upper canyon (not shown), the vertical mass flux is negative throughout both the realistic and the mountain wave only simulation consistent with a strong stationary wave descending the east side of the Continental Divide. This mass flux is of consistently greater magnitude in the realistic simulation as strong stable layers confine the surmounting flow to a lesser depth, thereby intensifying its wind speed overnight relative to the mountain wave only simulation. 


\section{Integrals of heating}

As expected the vertical integral (taken downward) of heating in time for the katabatic flow only simulation shows large, steady, cooling (Figure 7.7c). The values shown here are somewhat large (perhaps $150-200 \%$ of realistic values) because of the excessive cooling problem described in Section 6.4. Regardless, this behaviour is intuitive and shows significant cooling through the depth of the $\sim 650$ m deep katabatic layer. The mountain wave simulation also shows significant cooling in time, but through a far deeper layer. An analysis of the terms affecting potential temperature indicates that this is primarily due to advection. The cooler air to the west, as nudged into the outer boundary of the domain overnight, is advected to the east. Combining the nudging and radiative factor into the realistic case does not result in an additive relationship. That is, the realistic simulation shows characteristics of both sensitivity simulations. As in the katabatic flow only simulation, the most severe cooling is confined to the lowest atmospheric levels, showing that katabatic forcing should, indeed, exist, as we have shown previously. But more like the

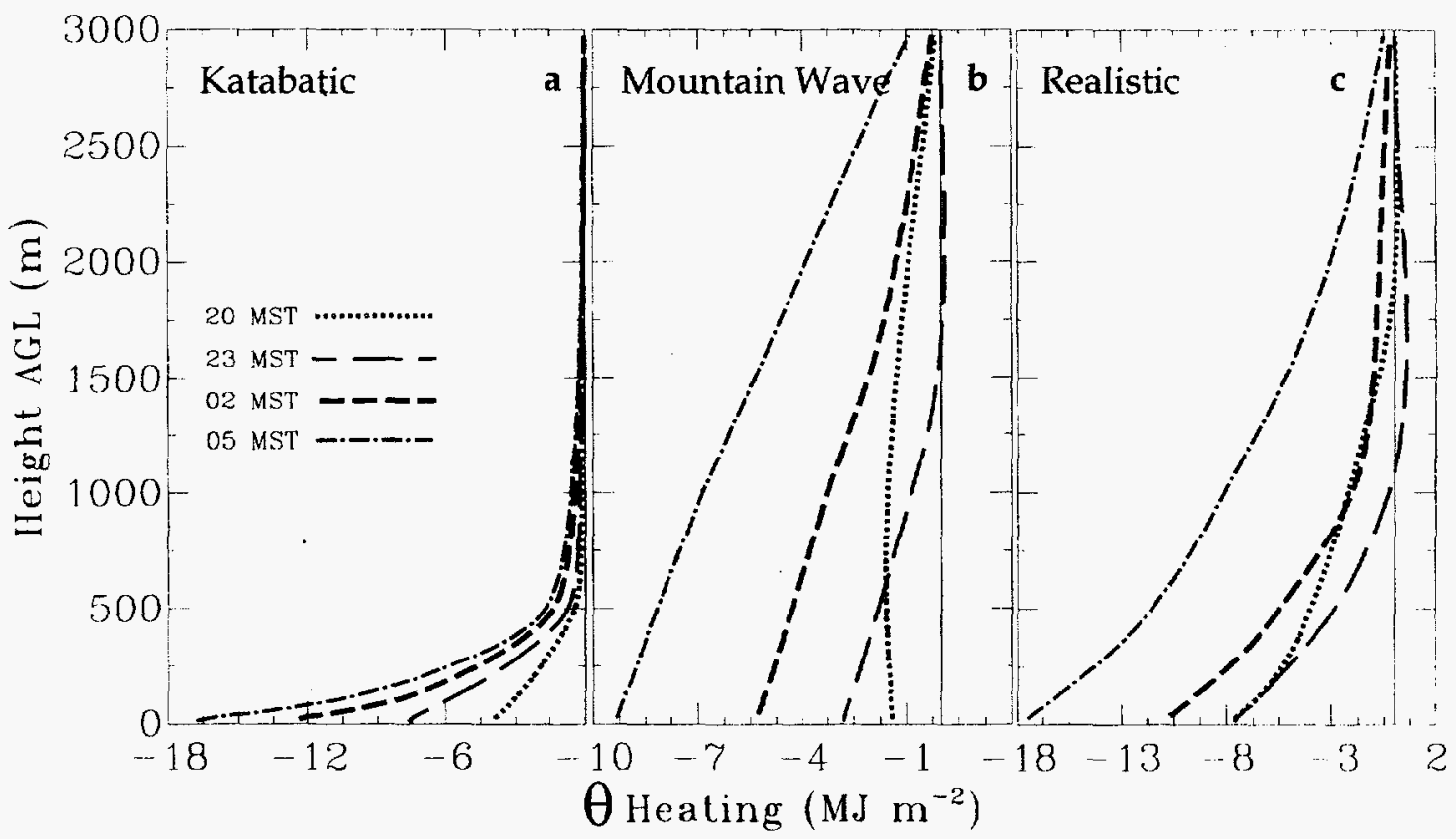

Figure 7.7 The same as Figure 7.5 but for heat content (megajoules $\mathrm{m}^{-2}$ ) integrated downward relative to the model start at 1700 MST. 
mountain wave simulation, this cooling extends to deeper layers, although not as cool as the mountain wave simulation itself at the highest altitudes. In fact, there is an enhanced (relative to the mountain wave simulation) region of slight heating at 2300 MST in upper levels. From this figure it is clear that the mountain wave has the overall effect of mixing cooling to a greater depth than would be achieved without mountain wave dynamics active. Conversely, radiative forcing strengthens the cooling in the lower layers of the atmosphere, increasing the stratification, and causing greater adhesion of the mountain wave to the topography. Because the strongest mountain wave momentum is thereby confined to a smaller depth, the resulting wind speeds are higher downwind of the Continental Divide, which further enhances the ability of the mountain wave to mix out cool air that otherwise would collect in the valleys.

\subsection{MODEL FORCINGS}

Although cross sections, time series of model variables and integrated fluxes, as described in the previous chapter and earlier sections of this Chapter, reveal a large amount of information about MKI, there is no better information about the dynamics of MKI than that which comes from the terms that comprise the equations of motion. To extract this information from a primitive equation numerical model such as RAMS is made most difficult by the volume of information that becomes available. For example, each value of $\mathrm{u}$-component wind has 5 components that create its value at the current timestep (when $\partial u / \partial t$ is applied to the $u$ velocity), advection, coriolis, pressure gradient, turbulence and various other model forcings (such as boundary conditions). In addition, the local time rate of change, $\partial u / \partial t$, is of interest, as well as a residual calculated by subtracting the individual forcings from $\partial u / \partial t$. To extract these forcing terms for each model 
variable of interest ( $u, v, w, \theta$, TKE and $\pi$ ) at each timestep, creates enormous model output - a gigabyte per model hour without difficulty. For the realistic topography simulations, over 30 horizontal points were selected for model forcings extraction every two minutes (every two $\Delta t$ on Grid 1 ) as shown in Figure 6.2e. This data rate was insufficient for spectral analysis.

\subsubsection{Lower canyon, $50 \mathrm{~m}$ above ground level}

Figure 7.8 demonstrates a primary result of this work. Lines of components of the local $u$ acceleration are plotted as a 4 point running average from 2 minute sampled data ( 8 minute averages). Figure $7.8 \mathrm{a}$ shows that the positive $u$ component of katabatic wind in the lower portion of Eldorado Canyon at $50 \mathrm{~m} \mathrm{AGL}$ is forced by the pressure gradient as balanced primarily by advection of weaker westerly momentum and somewhat more weakly by turbulent forcing. Small, positive acceleration of westerly flow occurs during the 1-4 hour period from model start, thereafter the flow is essentially balanced at this level. Note that the line indicating total local tendency, $\partial u / \partial t$ or 'TOTAL', is only slightly positive and, on the scale of this plot is hardly noticeable. This fact is a testament to the robust balancing act occurring in the evolution of katabatic flows. Throughout the latter 11 hours of the 12 hour period the pressure gradient force increases in response to continuous turbulent transfer of radiatively cooled air upward from the surface, with a strong increase from 6.5 - 8.5 hours. During this time, strong cooling occurs (as discussed earlier in Sections 6.5 and 7.2), and the horizontal pressure gradient force therefore increases as the local pressure at this elevation MSL becomes even larger than that in the free atmosphere at the same level.

Figure $7.8 \mathrm{~b}$ shows the model forcing on the $\mathrm{u}$-component of the wind at the same point 


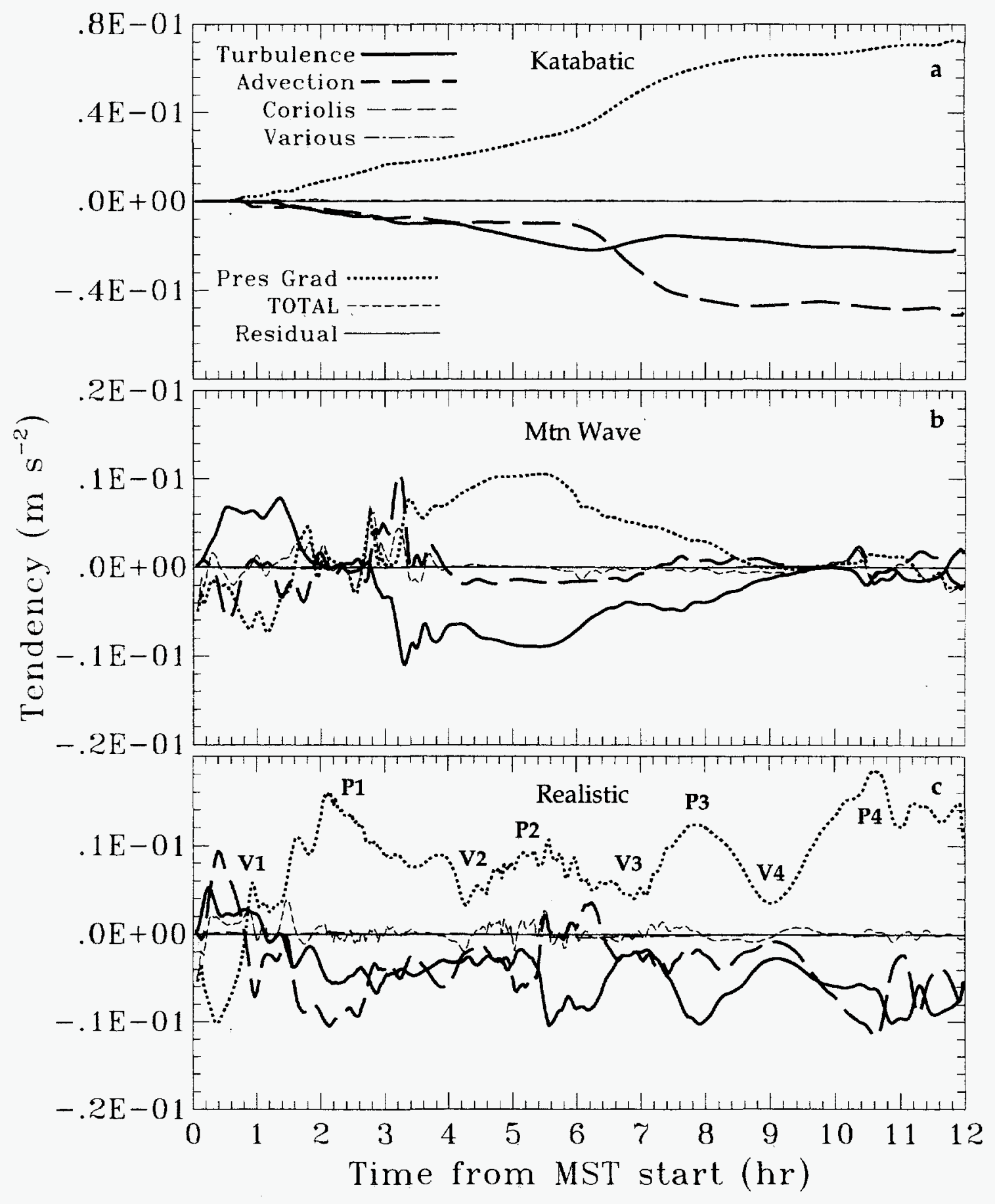

Figure 7.8 Terms of the conservation of $\mathrm{u}$-component momentum equation as calculated by RAMS for the 3-4 Sep 1993 case night for a) the katabatic flow only simulation b) the mountain wave only simulation, and c) the realistic simulation. 12 hours of data is presented in an 8 minute running average of 2 minute data for the lower canyon point (\#19 inFigure $6.2 \mathrm{e}$ ) at $50 \mathrm{~m}$ AGL. The P's and V's in panel $c$, indicate peaks $\left({ }^{\prime} P^{\prime}\right)$ and valleys $\left({ }^{\prime} V^{\prime}\right)$ in the pressure gradient force, and can be compared to the same indicators in Figure 7.9. 
but for the mountain wave only simulation. The magnitude of the forces, at their maximum, are approximately 6 times smaller than those in the $\mathrm{KFO}$ simulation after 6 hours (see Section 6.4). This is, in part, due to the excessively cold air that develops in the katabatic flow only simulation, allowing for a large horizontal pressure gradient force to develop. The mountain wave only simulation develops a nearly neutral low-level atmosphere driven by boundary mixing. Clearly, the varying flow over the Continental Divide causes a likewise variable flow condition in this portion of the canyon. Generally, the total tendency is larger and more noticeable than in the katabatic flow only simulation, with its sign determined by the local advective forcing. For most of the 12 hour period turbulence balances the pressure gradient force, with adjustments to existing advective acceleration. This balance is particularly evident during a relatively steady period from 4-8 hours. This period corresponds to increased westerly flow (see Figure 7.1b) and a somewhat larger horizontal pressure gradient therefore exists.

The combination of mountain wave and katabatic forcings creates, in Figure $7.8 \mathrm{c}$, a more variable state than exists with either forcing individually. This fact, in and of itself, shows that MKI affects the dynamics of both phenomena significantly and in a non-linear fashion. The pressure gradient forcing, for instance, is consistently large and positive after hour 1 , whereas the mountain wave only simulation is comparably large during only the 3 7 hour period. Whereas this 3-7 hour period in the MWO simulation shows a smooth behavior and flow increases in this region, with cooling the behavior becomes variable and erratic. Overall, similar to the katabatic flow only case, a force balance is struck between positive pressure gradient acceleration, and negative advective and turbulent acceleration. Time-height series of $u, w, \theta, T K E$ and radiative flux divergence (not shown) 
indicate that katabatic forcing dominated this level, despite the variability. As described in Chapter 6, this location (\#19 in Figure 6.2e) is where convergent v-component flows create a local high pressure region preventing classic katabatic flow development, even in the KFO simulation (unfortunately extraction points were not able to be altered once the simulations had begun). Note that the u-component of the divergence is too small to offset $v$ component convergence at this location. The fact that similar behaviour occurs in the realistic simulation, but not the MWO simulation, shows that katabatic forcing dominates in this region as well. Down canyon of this point, katabatic flow is evident in the realistic simulation, but it is of greater depth and resides in thermal atmospheric stability of lesser strength than in the KFO simulation. This more elevated structure is caused by larger mixing due to the varying overlying mountain wave, and verifies some of the conclusions from the idealized modeling.

Another interesting aspect of Figure $7.8 \mathrm{c}$ is the routine occurrence of a peak and valley in the pressure gradient force at a $\sim 2$ hourly interval. These meso- $\gamma$ scale features are labeled 'P1' through 'P4' and ' $\mathrm{V} 1$ ' through ' $\mathrm{V} 4$ ', respectively. It is clear, based on the relatively high magnitude of the pressure gradient force at $\mathrm{P} 1$ compared to that of the MWO simulation, that a combination of mountain wave pressure forcing and radiative induced pressure (katabatic) forcing is at work. As in the MWO simulation, near surface horizontal pressure gradients are induced by the overlying mountain wave; in the realistic simulation, these gradients are modified by radiative cooling. At P1, not only has cooler, denser air, begun to accumulate and create a katabatic-type horizontal pressure gradient, but also the surface pressure forcing caused by the vertical structure of the mountain wave, locally increases the near surface pressure. As a consequence, the combined effect leads to a local 


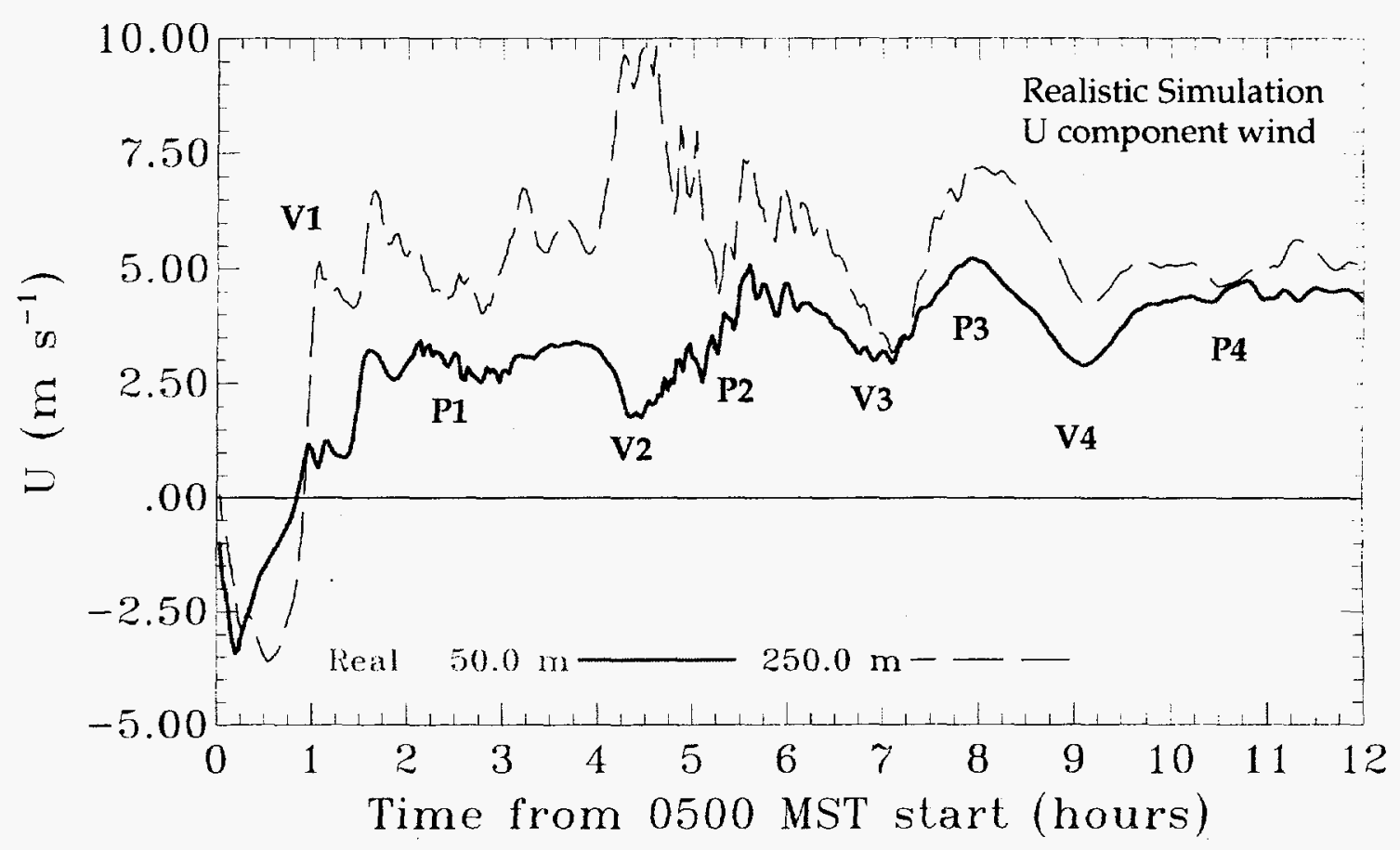

Figure 7.9 An expanded scale plot of the u component at the lower canyon location for 12 hours during the realistic simulation, also shown in Figure 7.1b. P's and V's correspond to the peaks and valleys of pressure gradient force shown in Figure $7.8 \mathrm{c}$.

maximum in the horizontal pressure gradient force. Low-level winds, however, only increase slightly because the mountain wave has separated from the topography, leaving relatively quiescent air beneath. Each of the labeled peaks and valleys on Figure $7.8 \mathrm{c}$ can be explained in a similar fashion as different surface pressure gradient effects due to the vertical mountain wave structure evolve over the top of this horizontal position in time.

Figure 7.9 is an expanded scale view of the u component time evolution plotted on Figure $7.1 \mathrm{~b}$ at the $50 \mathrm{~m}$ and $250 \mathrm{~m}$ AGL level of the realistic simulation. In this Figure the meso$\gamma$ scale rise and fall of the mountain wave influenced pressure gradient force (denoted by $\mathrm{P \#}$ and V\# in Figure $7.8 \mathrm{c}$ ) are reflected as $1-3 \mathrm{~m} \mathrm{~s}^{-1}$ meso- $\gamma$ scale fluctuations in katabatic wind speed, also denoted by P's and V's (an increase/decrease in wind speed occurring where the pressure gradient force is high/low).

One might ask whether the undulations in pressure gradient force shown in Figure $7.8 \mathrm{c}$ 
are caused by changes to katabatic forcing, since the interaction of mountain waves with katabatic flow is an inherently non-linear process. An alteration of the katabatic forcing would be reflected as oscillations of temperature that correlate to those in Figures 7.9 and 7.8c; but though some oscillations do exist (see Figure 7.4b), as would be expected for an evolving flow, these oscillations are quite small and not correlated to the PGF oscillations. Thus, we conclude that pressure forcing from an overlying mountain wave can drastically influence the otherwise steady pressure gradient forcing in katabatic flow (Figure 7.8a), inducing significant variability. The pressure gradient force in Figure 7.8c does not increase in magnitude throughout the 12 hour simulation, as seen in panel $a$, because the increased mixing prevents continuous cooling (see the $50 \mathrm{~m}$ AGL level in Figure 7.4b).

We also note that, similar to the idealized simulations, micro-timescale (order 1 minute) fluctuations in the near surface flow correspond to those periods where mountain wave breaking occurs above a particular horizontal location. Smoother evolution corresponds to times where mountain wave flow is not unstable. From Figure 7.9, these microscale fluctuations occur from $2-3.25$ hours and from 5 to $\sim 7$ hours, when wave breaking occurs above this point. From the above, it can be concluded that the near surface realization of the dynamic pressure forcing from the integrated vertical structure of an overlying mountain wave directly infiuences the development and evolution of katabatic winds, in support of the hypothesis of this dissertation.

\subsubsection{Lower canyon, $250 \mathrm{~m}$ above ground level}

At higher levels, such as $250 \mathrm{~m}$ AGL in the Lower Canyon (see Figure 7.10) the relationship of mountain wave pressure forcing to near surface winds is somewhat masked by the momentum of the mountain wave itself. At this level in the katabatic flow only case, 
Figure $7.10 \mathrm{a}$, where katabatic flow is protected from surface frictional influence by the stratified layers below, a primarily two-way force balance is found between the positive pressure gradient force and the advection of slower upstream air into the location. This balance classifies this flow as an advective-gravity flow in the Mahrt (1982) scheme. Aside from the steady growth of each part in the force balance, there is little variability in the various accelerations over time. This is reflected in the slowly increasing flow speeds in Figure $7.1 \mathrm{~b}$, to a nearly steady condition. In the mountain wave only simulation, where radiatively induced stable layers, and hence frictional decoupling, cannot occur, turbulence remains a significant factor in the force balance for $\mathrm{u}$-component winds (Figure $7.10 \mathrm{~b}$ ). The magnitude of the negative turbulent forcing (weakening flow due to turbulent mixing of slower flow into an area) for this altitude in the mountain wave only simulation is somewhat smaller than that at the surface, however, although the pressure gradient force is similar, such that a jet-like structure forms with stronger winds aloft from 3-8 hours. Depending on the speed of winds approaching this horizontal location, which depends on the incoming upstream flow impacting the barrier, topographic interaction and thermal structure, advection plays a variable role on the u-component tendency.

In the realistic case, Figure $7.10 \mathrm{c}$, a highly variable force balance is depicted, with many similar features to that of the $50 \mathrm{~m} \mathrm{AGL}$ plots in Figure 7.8. The first and foremost similarity, is the existence of peaks and valleys in the pressure gradient force in time. However, turbulence, which played a primarily negative role at the $50 \mathrm{~m}$ AGL level, here is occasionally negative but also positive for the 4-9 hour period. The $250 \mathrm{~m}$ AGL level is somewhat closer to the primary route taken by the highest momentum in the overlying mountain wave and thus is more easily effected by it. During this period, discerning what 


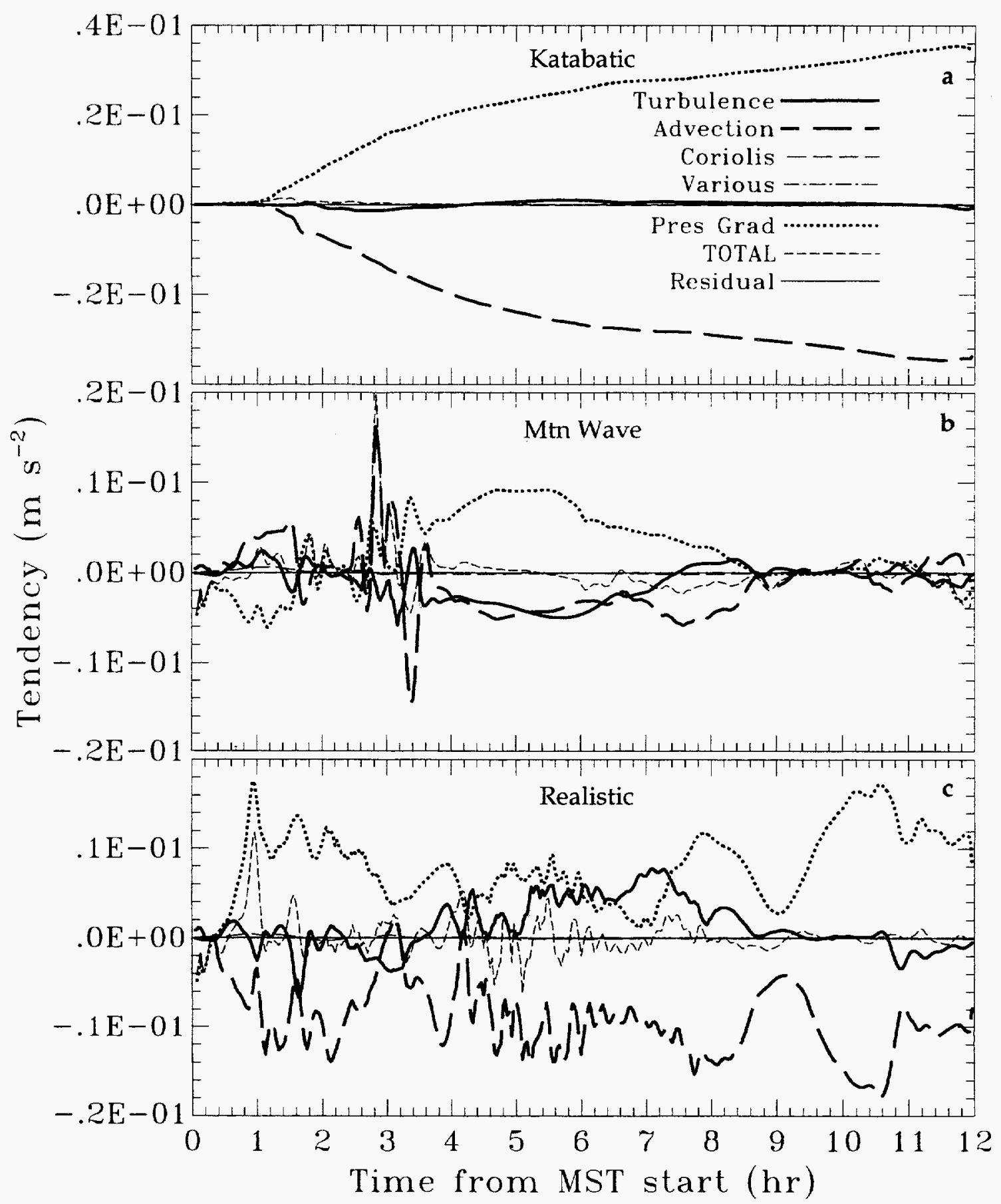

Figure 7.10 As in Figure 7.8 but for $250 \mathrm{~m}$ AGL (vertical level 15).

is katabatic flow and what flow is caused by mountain wave dynamics is nearly impossible. Clearly, some katabatic forcing is occurring due to the similarity in force balance between the katabatic flow only simulation and the realistic simulation at this lower can- 
yon, well sheltered location, but the wavelength and high momentum undoubtedly contributes to the flow here. Katabatic flow seems to be intermittently dominant depending on the strength and phase of the overlying mountain wave, with most domination occurring in the first 3 and last 3 hours of the 12 hours simulation.

\subsubsection{Upper canyon, $50 \mathrm{~m}$ above ground level}

In the upper canyon, dynamical evolution differs significantly from that in the lower canyon, most significantly by the role of the mountain wave. In Figure 7.11a, a near steady state is depicted for the katabatic flow only simulation at this horizontal point (\#30 in Figure 6.2e) after 5 hours. Because this portion of the canyon is oriented with a significant north-south component, and katabatic flows therefore have a large v-component, coriolis forcing on $u$ is notable compared to the east-west oriented lower canyon section. As westeast katabatic flow grows in strength from the Continental Divide slope on the west side of this location, and crosses the canyon axis, advection becomes a positive $\mathrm{u}$ forcing, compared with consistently negative forcing in the lower canyon at $50 \mathrm{~m}$ AGL (Figure 7.8a). Overall, the size of the forcing on $u$ is considerably smaller than that in the lower canyon for 2 reasons; 1 ) the upper canyon is more north-south oriented so that katabatic forcing is primarily in the $\mathrm{v}$ component (not shown), and 2) the lower canyon experienced excessive cooling in the latter 6 hours of the simulation, prior to that time lower canyon katabatic forcing is much smaller and realistic.

In the mountain wave only simulation (Figure 7.11b), upper canyon tendency evolution is consistent with flow dominated by mountain wave momentum, which is also called scouring. That is, this horizontal point lies in the topographically anchored, downward phase of the primary wave caused by upstream northwesterly flow, though somewhat pro 


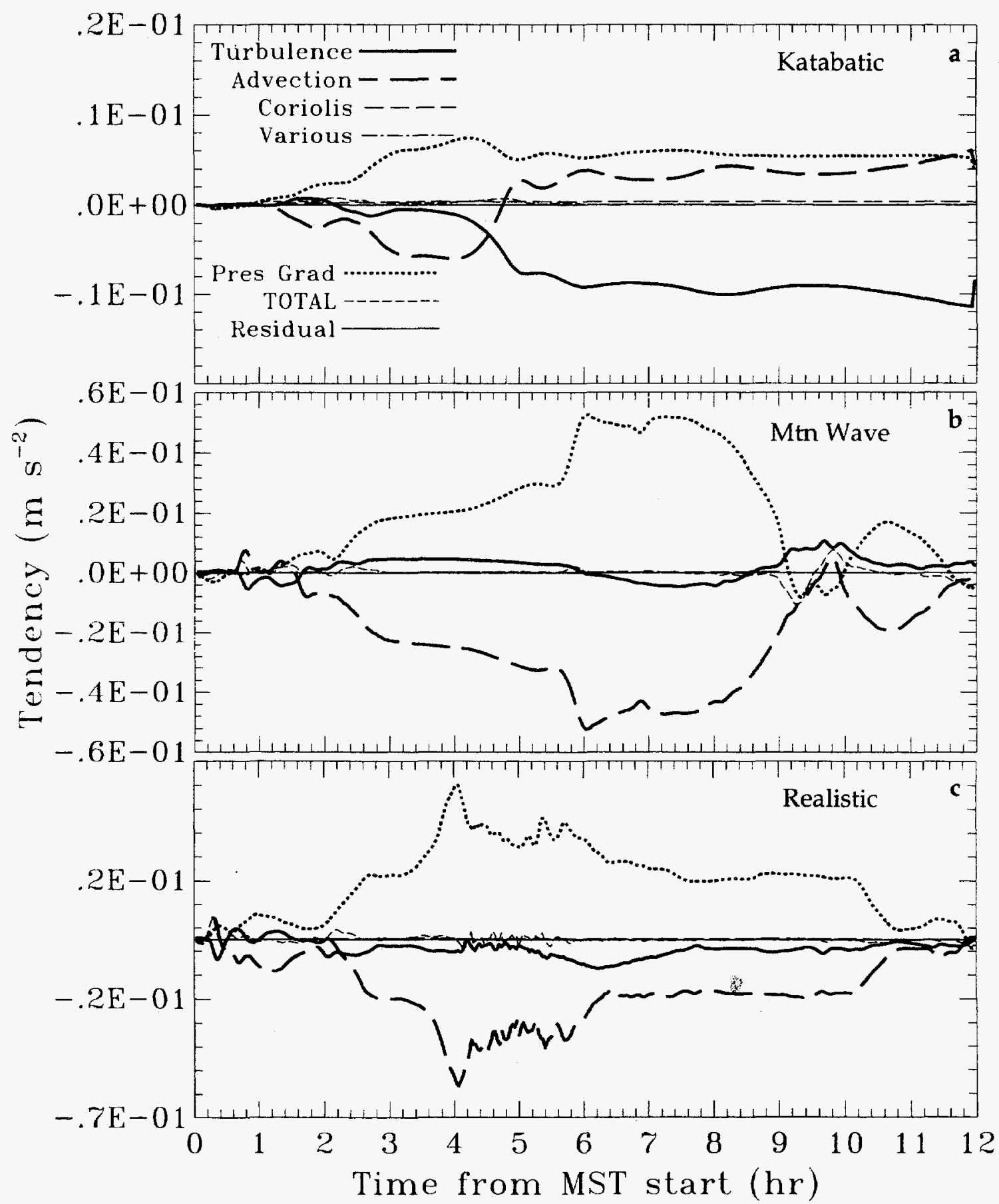

Figure 7.11 As in Figure 7.8 but for the upper canyon point (\#30 in Figure 6.2e) at $50 \mathrm{~m}$ AGL. -tected by the local canyon orientation. As the upstream flow surmounts the barrier and accelerates down the east side of the Continental Divide a positive horizontal pressure gradient force drives the flow. Positive forcing is also contributed from turbulent diffusion 
from 2 to 6 hours as strong shear induces mixing and therefore higher momentum air to be transferred down to this level. After the 8 hour point, weaker upstream winds exist, and pressure gradient forcing is reduced. As a consequence, winds temporarily lighten and become more variable, with turbulence playing a larger positive role. Toward the end of the simulation, upstream winds again increase causing a return to positive a pressure gradient force, and stronger, mountain wave induced winds.

The evolution of the $u$ component in the realistic simulation (Figure 7.11c) is somewhat similar to that of the MWO simulation, showing that mountain wave dynamics dominate in this upper canyon location. In Figure 7.11c, this fact is most easily recognized by the magnitude of the pressure gradient force driving u component flow. If the forcing were purely katabatic at this level, the KFO simulation shows that pressure gradient tendency would be able to achieve a maximum of $\sim 0.01 \mathrm{~m} \mathrm{~s}^{-2}$, whereas a value approximately 6 times this is achieved in the realistic simulation, similar to the mountain wave only simulation. The strong mountain wave momentum and shear prevents the development of stratification as strong as that in the katabatic flow only simulation. However, the stratification that does develop causes the mountain wave to be confined to a shallower depth and thereby adhere to the mountainside somewhat more than in the mountain wave only simulation without cooling. Thus, radiative cooling serves to increase the mountain wave momentum presence near the surface, allowing greater domination of the flow by the mountain wave than would otherwise occur.

\subsubsection{Plains east, $50 \mathrm{~m}$ above ground level}

On the plains east (Figure 6.2e, point \#8), where there is only a gentle slope, horizontal pressure gradients caused by cooling play a lesser role in forcing the $u$ component. This is 


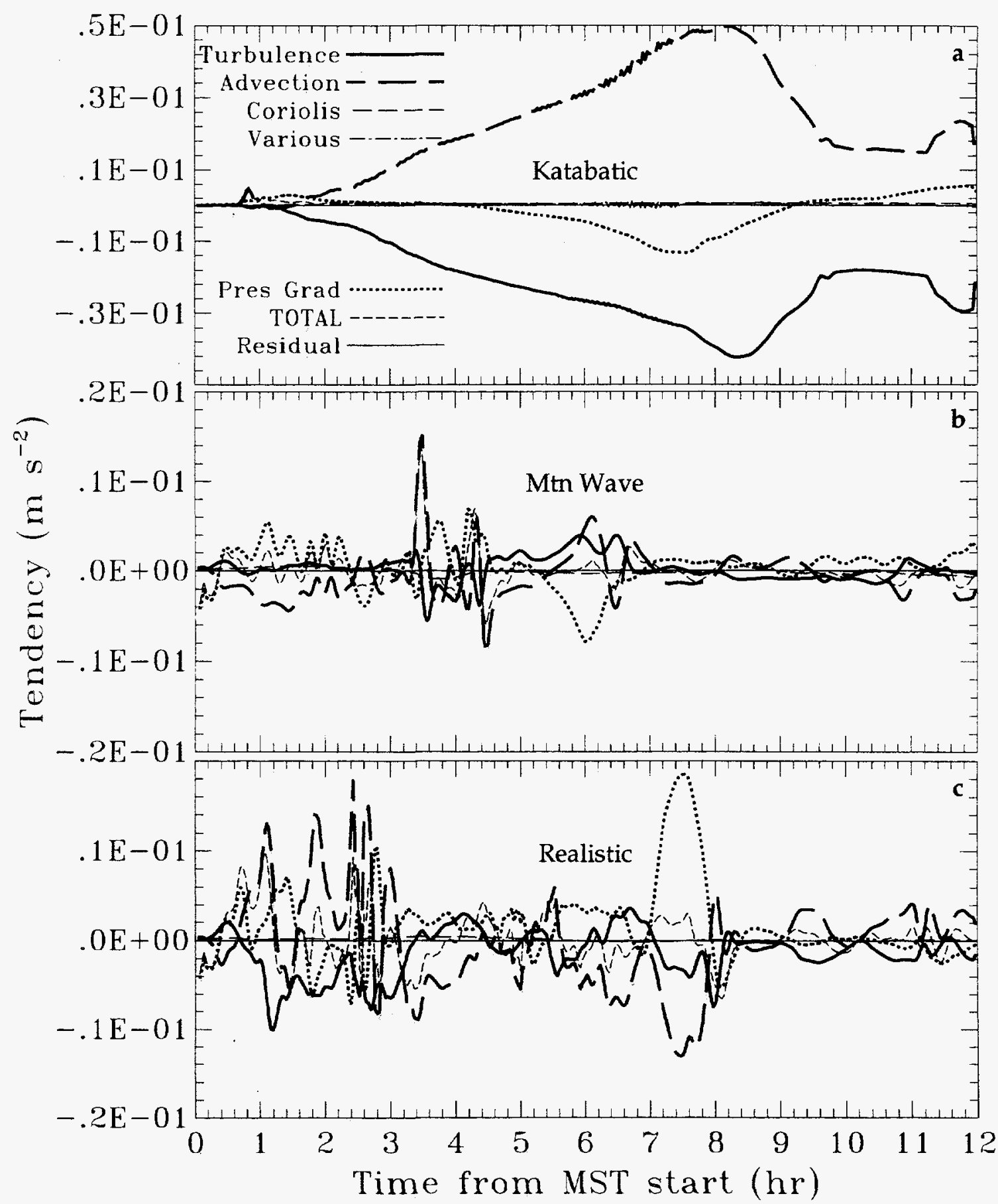

Figure 7.12 As in Figure 7.8 but for the plains east point ( $\# 8$ in Figure 6.2e) at $50 \mathrm{~m}$ AGL. shown foremost in Figure 7.12, panel $a$, where katabatic flows are driven by advection, rather than the pressure gradient force. Katabatic flows that form within Eldorado Canyon and along the general Front Range slope descend to the plains and flow over this point. 
Because of the lesser slope, strong surface cooling does not manifest itself as a strong horizontal pressure gradient force (i.e. nearby points in the horizontal are also cooled significantly). Katabatic flow speeds on the plains continually increase, though more slowly after 4 hours (Figure $7.1 \mathrm{c}$ )

In the mountain wave only simulation, where flow speed is generally less than $5 \mathrm{~m} \mathrm{~s}^{-}$ ',and weaker than either KFO or the realistic simulation, the dynamical balance is highly variable (see Figure $7.12 \mathrm{~b}$ ). As mountain waves of various wavelengths and amplitudes evolve throughout the twelve hour period, small timescale oscillations in near surface pressure gradient forcing, advection and turbulence are produced. The behaviour of these forcings is neither consistently positive nor negative and, as a consequence, $u$ wind speed at this level is generally light.

The variability of the mountain wave behaviour in the mountain wave only simulation translates to a highly variable flow state in the realistic simulation compared to the katabatic flow only case. As in the other portions of the canyon, the stratification at low levels is weaker than in the katabatic flow only simulation, because vertical turbulent mixing of heat is greater. This effect is augmented by the fact that katabatic flows advected over this region from Eldorado Canyon are not as cold as those in the katabatic flow only simulation. Katabatic flow does exist over the plains in the realistic simulation, however, as strong positive total tendencies for certain times during the 1-3 hour period are caused by advection of Eldorado Canyon katabatic flow over the region. This katabatic flow is somewhat deeper than that of the katabatic flow only simulation because cold air is mixed to a deeper level, causing deeper katabatic forcing. The overlying mountain wave therefore has the effect of influencing the depth of katabatic flow over the plains, as well as its 
strength and jet height. The variability in the forcing of this flow on the plains translates to far more variable wind speeds in the katabatic flow. This variability is evident in the $\mathrm{v}$ component of the wind as well (not shown), and causes a much larger standard deviation of horizontal wind direction than in the katabatic flow only case.

\subsection{SUMMARY}

In this chapter high resolution time series of meteorological variables, vertical integrals of various quantities and $\mathrm{u}$ equation of motion tendencies have been discussed for each of the three realistic topography simulations. Three locations within the domain were identified and used as examples during this discussion; 1) an upper Eldorado Canyon grid point just a few kilometers east of the Continental Divide, meant to represent a point heavily influenced by mountain wave momentum, 2) a lower Eldorado Canyon grid point approximately $2 \mathrm{~km}$ west of the canyon mouth, representing a well topographically sheltered, heavily katabatic flow influenced, location, and 3) a plains point about $4 \mathrm{~km}$ east of the Eldorado Canyon mouth.

The time series of meteorological variables showed that near surface flows, and specifically katabatic flows, were far more variable when influenced by an overlying mountain wave, as hypothesized would be the case in Chapter 1 . Further, some of this variability was due to the evolving atmospheric stratification as the night progressed which, as it increased, altered the wavelength and amplitude of the topographically forced wave. Depending on the non-linear evolution of stable layers, a very long wavelength wave could be produced with no additional down wind perturbations or, alternatively, a shorter wavelength wave with additional downstream undulations, or some other hybrid, could occur. These conclusions were further supported by vertical integrals of $\mathbf{u}$ component and 
vertical mass flux and heating in time. The u-component mass flux integrals showed that the return flow that develops locally in the katabatic flow is masked when mountain wave momentum dominates aloft. A local mass balance would therefore have to be reached by daytime heating and subsequent upslope flows during the daytime or by other means. Vertical mass flux integrals showed that mountain wave momentum is partly contained by thermal stratification, resulting in net downward mass flux and greater speeds in the accelerated portion of the wave. Heating integrals were used to show the importance of radiative cooling to the lower levels of the atmosphere, but also the importance of additional mixing by the mountain wave in deepening the cooled layer.

Time series of the accelerations on the $u$ component wind verified an important aspect of the hypothesis - that surface pressure undulations caused by the evolving overlying mountain wave can increase the variability of surface based katabatic flow. Changes in time of the horizontal pressure gradient acceleration were caused by the changing surface pressure perturbation of the evolving mountain wave. The vertically integrated dynamic pressure effect due to the mountain wave (a consequence of the varying wave structure with height) caused an alteration to the local pressure gradient force, sufficient to result in a 1-3 $\mathrm{m} \mathrm{s}^{-1}$ change in katabatic flow speed. This effect was most evident in the near surface layer (50 m AGL) where local stratification and topographic sheltering prevents katabatic flows from being overwhelmed by mountain wave momentum, and contained an a meso- $\gamma$ scale ( 2 hourly) oscillation linked to mountain wave evolution. At $250 \mathrm{~m}$ AGL, the relationship was still valid but more masked by the strong gradients there. Where mountain wave breaking occurred, the wind speed displayed microscale (order 1 minute) variability in time due to rapid surface pressure (and therefore pressure gradient) changes. 
Nocturnal low-level winds often could not easily be classified as primarily dominated by either katabatic flow or mountain wave momentum, emphasizing the point that these two complex terrain phenomena are, in fact, inseparable. 


\section{CHAPTER 8}

\section{Conclusions}

\subsection{OVERVIEW}

Due to the strong thermal stratification they develop in, katabatic flows are generally considered to be a relatively turbulence-free atmospheric phenomena sheltered from excessive external influence. However, greater-than-expected variability in katabatic flows, as expressed through tracer releases and their observed dispersion within these flows, was found during the ASCOT field program near Rocky Flats, Colorado, in 1993 (Poulos and Bossert 1995). Toward understanding the causes of variability in complex terrain katabatic flows, it was hypothesized that a frequently occurring complex terrain phenomena, mountain waves (also known as topographically forced internal waves), would impact the variability and evolution of katabatic flow. It was further hypothesized that this interaction would be complicated by the mutual evolution of the two phenomena, which is inherently non-linear.

Previous work on katabatic flows has typically attempted to isolate either katabatic flow or mountain waves from external forcing mechanisms, with a few exceptions, as described in Chapter 2. In general, those papers describing the interaction of katabatic flow with other phenomena have found that external influence on katabatic flow is significant. Works such as Barr and Orgill (1989) and Orgill et al. (1992) have shown, using observa- 
tions, that cross-canyon ambient flow speed is correlated to changes in katabatic flow depth. Ambient wind that opposes katabatic flow direction has been found to scour katabatic flow completely, if strong enough, but otherwise can enhance katabatic forcing (Arritt and Pielke 1986, Neff and King 1989, Orgill et al. 1992). Mursch-Radlgruber (1995) suggested that the enhanced katabatic flow speed may be partly caused by the dynamics of mountain wave pressure forcing. Coulter and Gudiksen (1995) have shown that the response of katabatic flow to an aiding flow is to strengthen at lower external wind speeds, but to slow at higher external wind speeds.

A case study night where mountain wave interaction with katabatic flow (MKI) was likely, was chosen from the ASCOT 1993 field program and is described in Chapter 3. Analysis of this case night, 03-04 Sep 1993, showed that upstream flow approximated a Fr $=0.42$ flow overnight, while observations showed katabatic flow along the Front Range of the Colorado Rocky Mountains. A Froude number climatology of the region showed that the typical atmospheric structure along the Front Range lies within the well known nonlinear regime of mountain wave flow $(0.1<\mathrm{Fr}<0.9)$, so this night is considered normal. Observations were insufficient to ascertain much information about MKI, so the investigation turned to numerical modeling. After verification of model capability in Chapter 4, idealized (Chapter 5) and realistic simulations were completed and analyzed (Chapters 6 \& 7), using the Regional Atmospheric Modeling System. Conclusions from the sum of this effort are summarized below. The majority of these conclusions concern the effects of the eastward flowing component of the mountain wave on similarly flowing katabatic flow (aiding flow), since this is the typical regime along the Front Range of Colorado, although the idealized simulations do consider the upstream barrier slope katabatic flow and oppos- 
ing flow.

\subsection{UNDISTURBED KATABATIC FLOW}

In order to establish the basic behaviour of katabatic flows before tackling the more complex problem of $\mathrm{MKI}$, they were investigated without external influence, and a number of interesting conclusions were made.

1. The existence of weaker katabatic flow upstream is crucial to keeping katabatic flow speeds bounded, through advection, while turbulent levels are low. Turbulent diffusion, alone, is insufficient to offset the positive acceleration caused by increased cooling over time, except at the lowest model level.

2. A true steady state (temporally unchanging spatial configuration) is never achieved in undisturbed katabatic flow due to constantly changing atmospheric thermal stability. The flow does eventually reach a state that changes much more slowly (a 'quasi-steady state') than in the initial development stages, however.

3. After the development stage, a quasi steady state is achieved in katabatic flow when the adiabatic warming caused by downslope advection reaches a sufficient strength (for a given thermal stratification) to offset continuous cooling by radiative and turbulent processes. This requirement is met when katabatic flow at a particular slope location achieves a wind speed of sufficient magnitude.

4. At this quasi steady state, katabatic flow increases in depth downslope because potentially warmer air flowing downslope remains above potentially cooler air very near the surface in the downslope direction. Furthermore, increasing shear in the katabatic layer, as katabatic jet speed increases downslope, causes greater mixing of cool air upwards and subsequent deepening of the katabatic flow at lower elevations.

5. At quasi steady state, katabatic flow speed increases downslope because an air parcel at a given downslope location has accelerated for a longer period than those above it. Upslope locations achieve quasi steady state before downslope locations.

6. Gravity waves can be generated by shear between the katabatic flow jet and return flow or weak flow aloft. Gravity waves develop preferentially in this region, rather than below the jet, because thermal stratification is weaker above the jet, resulting in a 
higher chance for instability. If the shear instability is great enough Kelvin-Helmholtz waves will develop.

7. Return flow occurs in katabatic flow, though it may be masked by other atmospheric phenomena under normal circumstances.

\subsection{The Interaction of Katabatic Flow and Mountain Waves}

\subsubsection{Turbulent effects}

The phrase 'turbulent effects' is used here to encapsulate those mountain wave induced effects on katabatic flow that occur through diffusion or mixing processes, most often as regards thermal stratification. ASCOT observations, previous work and numerical simulations show that katabatic flows develop within strong (i.e. $\partial \theta / \partial z>10 \mathrm{~K} \mathrm{~km}^{-1}$ ), surfacebased thermal stratification which, itself, develops because of cooling driven by radiative flux divergence and turbulent mixing. Since this cooling is also responsible for the buoyancy forces that drive katabatic flow, the depth of this cool air, insofar as it is cooler than air in the free atmosphere at the same altitude, defines the depth of the katabatic flow layer. Therefore, alterations to this thermal structure by external influences such as mountain waves, cause changes in katabatic flow depth and strength.

Compared to katabatic flow in an undisturbed atmosphere, this investigation has shown that when a mountain wave is present, thermal stratification associated with katabatic flow is weaker and often deeper, on each side of the barrier (see Figure 8.1). As a consequence of this deeper layer, katabatic flows are also deepened, in most cases. Correspondingly smaller differences in temperature between the katabatic layer air and the free atmosphere cause the katabatic flow jet speed to decrease, and its vertical location to possibly increase slightly (this effect is somewhat exaggerated in Figure 8.1). However, if mountain wave flow is strong enough in a particular region of interest, mountain wave momentum will 
Katabatic flow without mountain wave

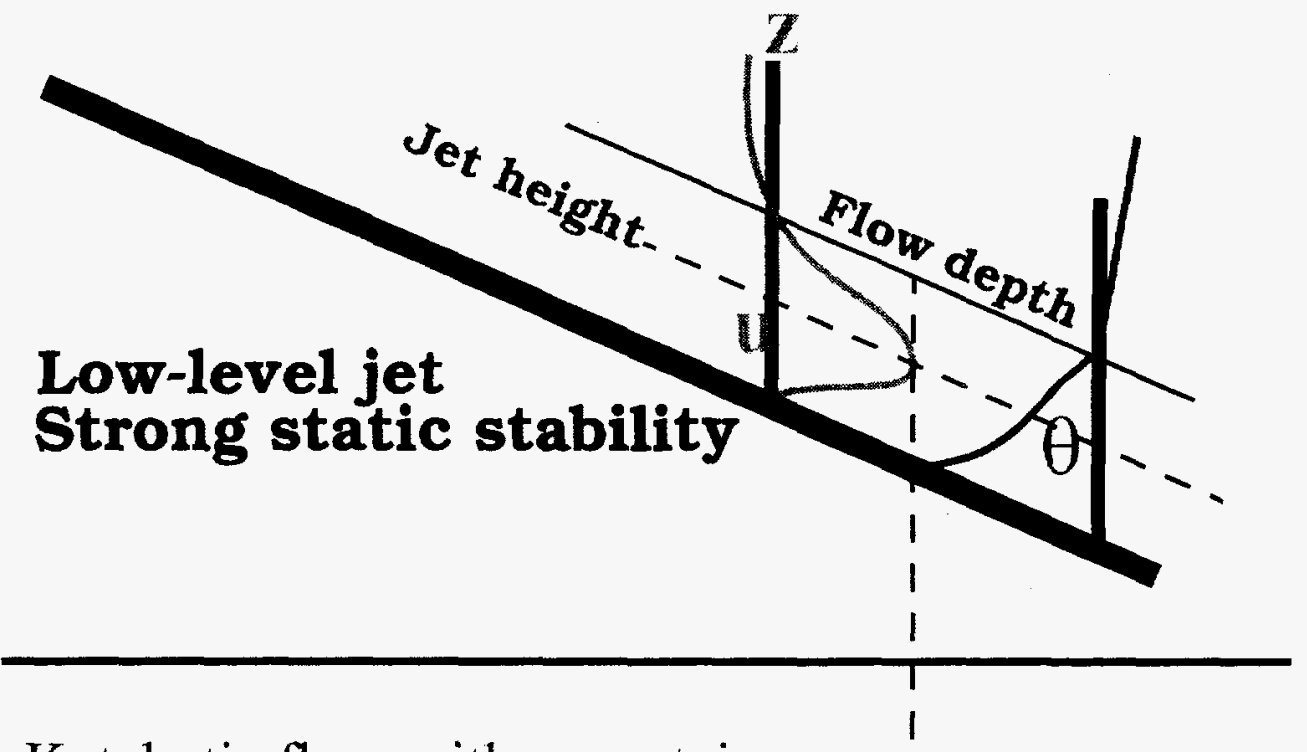

Katabatic flow with mountain wave,

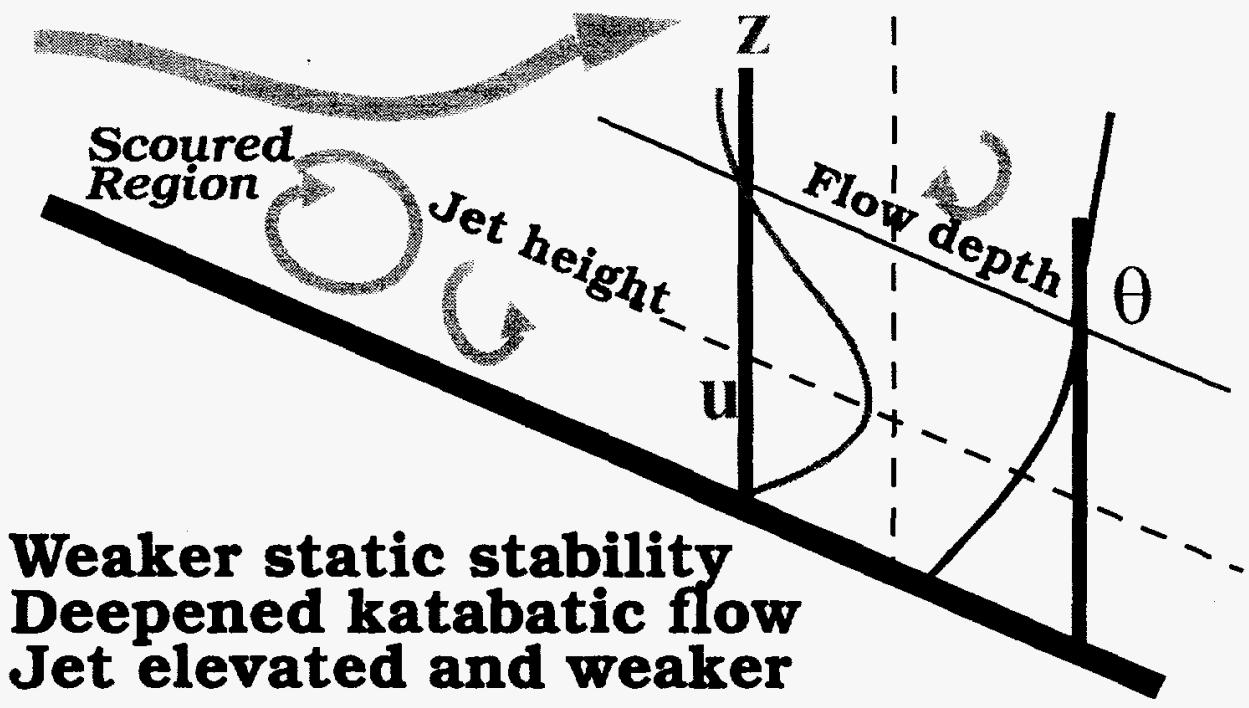

Figure 8.1 A schematic diagram of the influence of a mountain wave descending into lee side katabatic flows. The upper diagram shows the katabatic wind speed and static stability without mountain wave influence (see also Figure 1.1). The lower diagram indicates the katabatic flow structure with a mountain wave; upper slopes are scoured while the vertical structure of the katabatic flow on lower slopes is altered significantly.

completely dominate the near surface atmosphere, and scour katabatic flow features (Figure 8.1, upper slope of lower diagram). Cooling still occurs in this instance, however, though the stratification in the scoured zone is primarily caused by the strong subsidence in the downward phase of the mountain wave. The scoured zone can be defined by the dis- 
tance downslope to the separation point of the mountain wave (where the topographically anchored portion of the wave again leaves the surface). Thus, for higher Froude number, scouring proceeds to a greater distance downslope. Even in the case of $\mathrm{Fr}=1.0$; a case where theory states that the mountain wave will descend the entire slope length, katabatic flow can develop in strongly cooling lower altitudes if stratification becomes strong enough to prevent mountain wave penetration. For $\sim 0.40<\mathrm{Fr}<1.0$, scouring is still significant along the upper slopes of the barrier, but below the separation point flow is quiescent enough to allow the development of katabatic flow. The more topographically sheltered a location is, the more likely katabatic flow is to develop. At low $\operatorname{Fr}(=0.25)$, it has been found that the mountain wave dynamics are not powerful enough to scour katabatic flow except at the very highest altitudes. In fact, the mountain wave flow and katabatic flow become inseparable. Rather than separating from the mountainside, the wave flow merges with that of the katabatic flow due to strong stratification. Though the katabatic flow appears to actually exceed its undisturbed magnitude in this case, since the flows are inseparable, it is difficult to ascertain how the additional momentum is being imparted to the slope flow.

\subsubsection{Mountain wave induced pressure effects on katabatic flow}

An intriguing result of this study is that pressure effects caused by the evolving mountain wave can alter katabatic flow speeds, even if the mountain wave is propagating downstream, elevated above the katabatic flow (see Figure 8.2). For a given atmospheric condition, mountain wave flow will develop a complicated wave structure downstream of the barrier. In the immediate vicinity of the barrier it is well-known that the upstream side of the mountain will develop a surface high pressure whereas in the lee there will be rela- 


\section{MKI Dynamic Pressure Effects}

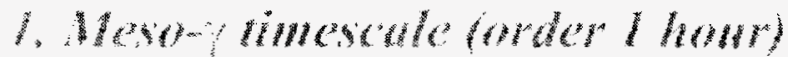

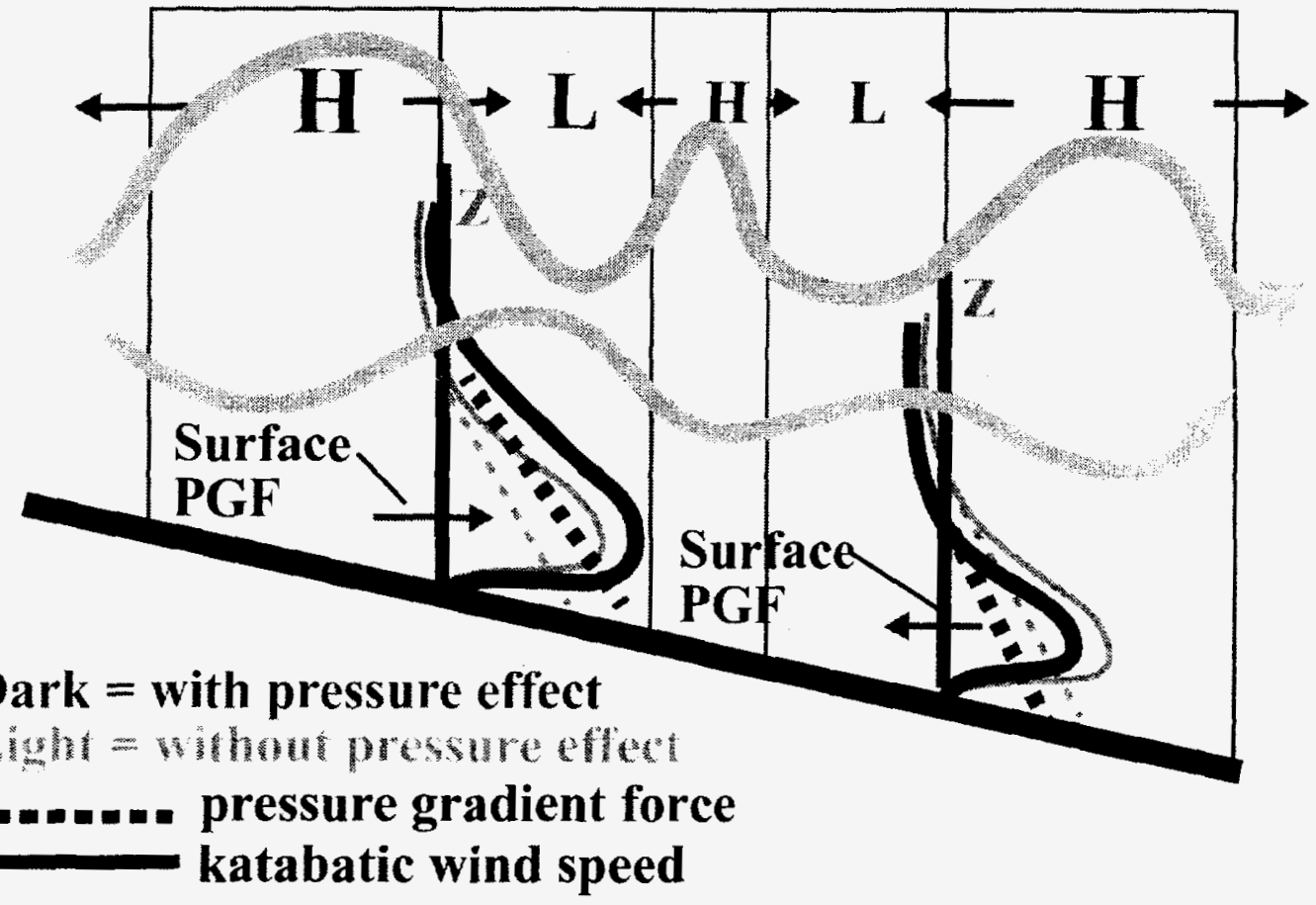

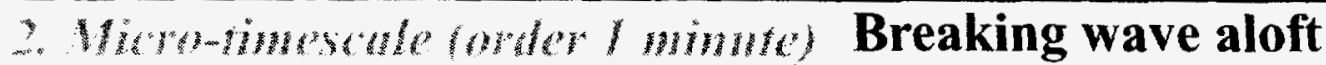
causes rapid pressure
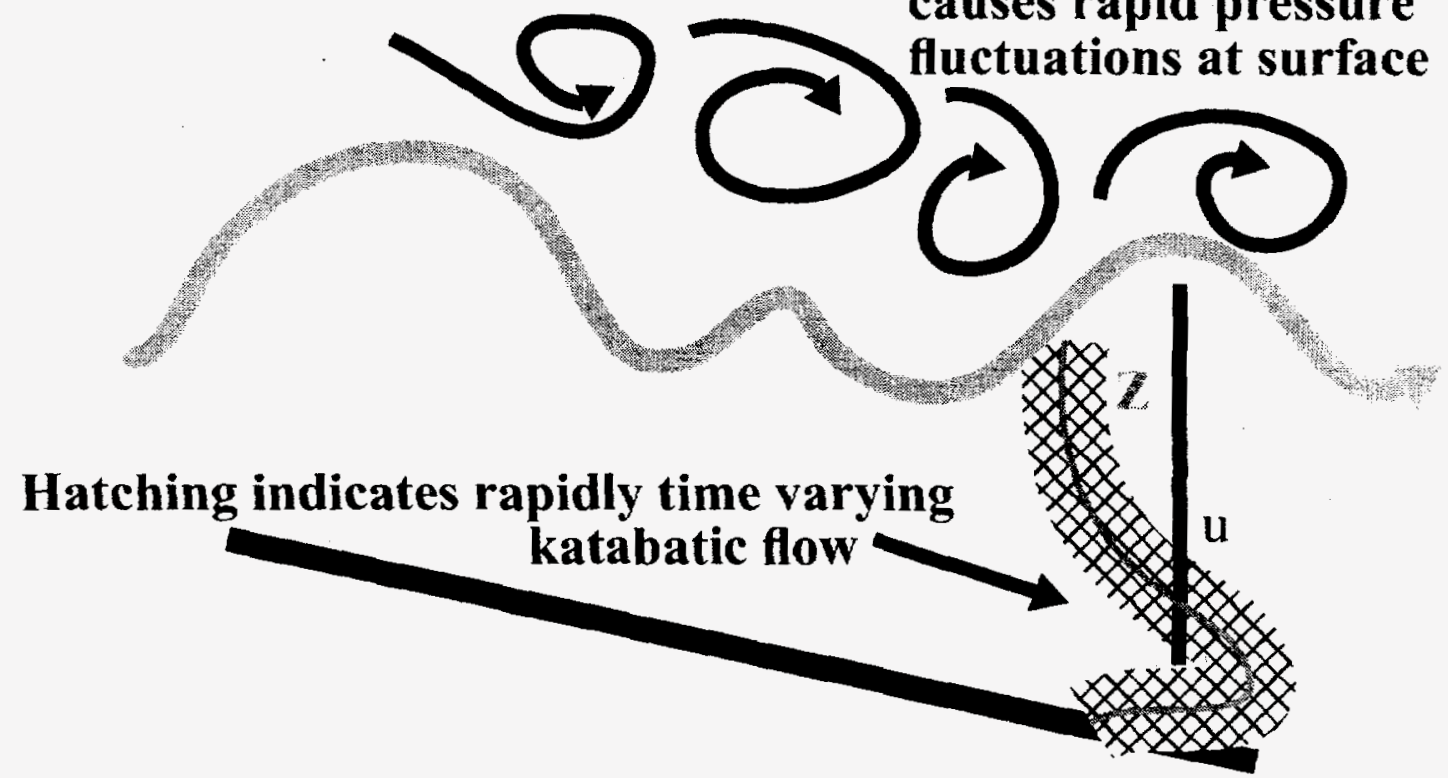
fluctuations at surface

Figure 8.2 Schematic diagrams of the two dynamic pressure effects of mountain waves on katabatic flow. The upper diagram shows that, depending on location, katabatic flow can be either strengthened (upper slope case) or weakened (lower slope case), due to the integrated column pressure structure of the mountain wave. The lower diagram shows that a breaking mountain wave aloft causes rapid pressure fluctuations which, in turn, causes rapid katabatic flow fluctuations. 
tively low pressure. Further in the lee, however, after the mountain wave separates from the barrier, any number of gravity wave structures can exist in the vertical, from strong, ducted, lee waves to gently propagating evanescent waves depending on the vertical profile of temperature and wind. Since the effect of this complicated wave structure on the surface pressure is determined by the vertically integrated pressure disturbance through the column above a particular point, the mountain wave induced surface pressure effect will vary with horizontal location. This effect can cause horizontal pressure variability on the order of $100 \mathrm{~Pa}$. Since katabatic flow is a surface-based, pressure gradient dependent phenomena, it is affected by these alterations of surface pressure gradient caused by the mountain wave, even if the wave undulations are elevated above the flow (see Figure 8.2). Note that in the upper diagram of Figure 8.2 that the column pressure changes are indicated as discrete highs $(\mathrm{H})$ and lows $(\mathrm{L})$ for clarity; in nature the pressure forcing is continuous in space. Furthermore, as the mountain wave evolve, the downstream wave structure, and therefore surface pressure perturbation, will change. Simulations have shown that katabatic flow speeds can be forced to vary by $1-3 \mathrm{~m} \mathrm{~s}^{-1}$ through this effect over meso- $\gamma$ (order 1 hour) timescales. It has also been found that high frequency oscillations in surface flow can be generated by the micro-timescale (order 1 minute) pressure perturbations induced by breaking waves aloft as illustrated in the lower diagram of Figure 8.2. Clearly, this effect is not limited to katabatic flow only, as any surface flow will be influenced by these pressure effects.

\subsubsection{The effect of evolving thermal stratification on mountain waves}

One of the causes of an evolving mountain wave pattern, in addition to changes in upstream flow characteristics, has been found to be continually changing nocturnal stratifi- 
cation. As we know from the use of Fr or the Scorer parameter to describe mountain wave flow, thermal stratification has a direct effect on mountain wave structure. Intuitively, increasing thermal stratification due to radiative cooling overnight should increase $\mathrm{N}$ and therefore lower Fr, causing the separation point of the mountain wave to move upslope. For $\mathrm{Fr}>\sim 0.6$ this was found to be the case. However, for lower $\operatorname{Fr}(<\sim 0.6)$ the intensifying low-level stratification was found to 'capture' the mountain wave flow causing it to adhere to the mountainside to a greater extent than otherwise. For the mountain wave produced by $\mathrm{Fr}=0.25$, this effect causes the flow to adhere to the mountain almost to the lowest topography.

\subsubsection{Their mutual overnight evolution}

The author wishes to emphasize the complicated evolution that exists when radiatively driven katabatic flow and mountain waves exist together. Oftentimes during the overnight period near the surface these flows are inseparable. The mountain wave, rather than being a steady state feature, is constantly changing due to time-varying upstream flow speed and direction, and local stratification. Evolving local stratification, which produces the negatively buoyant atmospheric structure required for katabatic flows, is altered by the level of mixing induced by overlying mountain wave flow. Consequently, katabatic flow structure is altered in a non-linear fashion depending on the interplay of all these causes of variability. The result is a far more variable stable nocturnal boundary layer in complex terrain than has been generally understood to exist.

\subsection{Other Conclusions}

\subsubsection{Modeling Alpine Phenomena}

It has been found that a crucial step prior to the investigation of a particular meteorolog- 
ical phenomena is scale analysis. For the given horizontal and vertical scale of a meteorological feature, at least 8 - 10 grid spacings should be used over that scale for proper resolution. If the development of this feature depends critically on an early stage where its scale is still smaller, that scale should be used to determine grid spacing. In complex terrain, and particularly for realistic katabatic flows, an appropriate terrain scale may also determine required grid spacing. Using this technique, it has been found that the rule-ofthumb criteria for modeling in steep topography (see Section 4.3.1) can be greatly exceeded without detrimental effects on the modeling result.

\subsubsection{Froude Number Climatology}

A climatology of Fr relevant to the Front Range of the Colorado Rockies was completed for the 28 year period 1966-1993 based on archives of Grand Junction, CO soundings. This climatology indicated that westerly flow existed in the lowest $2000 \mathrm{~m}$ below barrier height ( $4000 \mathrm{~m} \mathrm{MSL}$ ) for $\sim 80 \%$ of all days. Using either total wind or the $u$ component to calculate Fr, the average Fr was found to be predominantly in the non-linear regime of mountain wave flow with $\mathrm{Fr}_{\mathrm{u}}=0.31$ and $\mathrm{Fr}_{\mathrm{v}}=0.63$, where $\mathrm{Fr}_{\mathrm{u}}$ is calculated using the $\mathrm{u}$ component of the wind and $\mathrm{Fr}_{\mathrm{v}}$ is calculated with the total wind (see also Chapter 3). $\mathrm{Fr}$ was higher, on average, at $00 \mathrm{Z}$ than at $12 \mathrm{Z}$ by $0.15-0.20$, but this difference depended greatly on season. In winter, the $00 Z-12 Z$ Froude number difference was nearly zero due to the lack of solar insolation during the day, whereas during warmer months this difference exceeded 0.30. Additional details can be found in Appendix B.

\subsubsection{Truncation Errors in $\sigma$-coordinate models}

Due to the steep topography present in the Front Range of the Colorado Rockies and therefore in the case study modeling domain, an investigation was launched into the sig- 
nificance of truncation errors in the $\sigma$-coordinates used in RAMS. It was found that these errors could be greatly alleviated by using perturbation quantities (from a base state) in the turbulent parameterization. This technique is most easily applied to small spatial and temporal scale domains where the base state can be assumed invariant. For large scale simulation or long time scale simulations, the base state cannot be assumed invariant or else perturbations will become extremely large in time. However, in large spatial scale simulations topography seldom can be resolved sufficiently to create topographic slopes of concern. Particularly in idealized simulations, sending perturbation quantities to the turbulent parameterization will reduce errors. The implementation of the Mahrer (1984) scheme for the reduction of horizontal pressure gradient errors was found to reduce the maximum error by over $20 \%$ in limited tests. If applied to terms other than the pressure gradient, a greater improvement may be found. The primary difficulty in this implementation was the calculation of the horizontal gradient at a topographic interface where interpolation and extrapolation, or other techniques, are required.

\subsection{SUGGESTIONS FOR FUTURE RESEARCH}

-- The parameterization of dispersion quantities in the stable, nocturnal atmospheric boundary layer is known to be a significant source of error in dispersion models. In general, these parameterizations do not create sufficient horizontal dispersion in the thermally stable case as found, for example, in Poulos and Bossert (1995). Since these parameterizations are based on nearly flat terrain experimental data, the results of this dissertation strongly suggest that this error may, in part, be caused by the improper recognition of external influences on the variability of the stable boundary layer. An interesting research problem would be to implement the effects of this mountain wave induced variability into 
stable layer parameterizations.

-- Another interesting research topic would be to investigate the influence of gravity waves on the variability in the stable, nocturnal boundary layer over topography of much less complexity. As one might expect, there exists a continuum of gravity waves propagating throughout the atmosphere - they are not restricted to terrain as severe as that investigated in this dissertation. It can therefore be hypothesized that gravity waves, and their associated perturbations to meteorological quantities, would also influence the stable boundary layer over most every land surface.

-- In this work, a major cause of stable layer variability in complex terrain has been shown to be gravity wave behaviour downstream of a mountain barrier, though it is likely that numerous other factors contribute to this variability as well. Some of these factors, such as surface roughness, soil parameters, degree of cloudiness and vegetation, have been previously investigated, as described in Chapter 2, but a general understanding of them does not exist at present.

-- Some steps toward a comprehensive understanding of undisturbed katabatic flows have been taken in this work, but many aspects of these flows remain poorly understood. In particular, verification of why the katabatic flow jet appears at a given elevation should be pursued. The role of internally produced gravity waves on stable layer mixing, variability and 'turbulent burst' frequency is also an intriguing problem. Only an intensive complementary measurement and modeling program could resolve these issues satisfactorily. -- On the issue of truncation errors in terrain-following coordinate models, more effort to reduce errors could be fruitful. It would be particularly useful to utilize a known analytical solution for flow over relatively steep topography in comparisons with various model 
formulations to ascertain how horizontal gradient errors might be minimized. While zero initial wind simulations, such as those completed here, serve a definite purpose in extracting and analyzing the errors, the quiescent environment is hardly realistic. A modeling technique that gives a high quality performance under more atmospherically relevant conditions, in addition to the simple quiescent case, should be sought. 


\section{$\underline{\text { References }}$}

1. Allwine, K.J., 1993: Atmospheric dispersion and tracer ventilation in a deep mountain valley. J. Appl. Meteor, 32, 1017-1037.

2. Anderson, G.E., 1971: Mesoscale influences on wind fields. J. Appl. Meteor, 10, 377386.

3. Angell, J.K., D.H. Pack and N. Delver, 1969: Brunt-Vaisala oscillations in the planetary boundary layer. J. Atmos. Sci., 26, 1245-1252.

4. Arakawa, A., 1966: Computational design for long-term numerical integration of the equations of fluid motion: Two-dimensional incompressible flow. Part I. J. Comput. Phys., 1, 119-143.

5. Arritt, R.W. and R.A. Pielke, 1986: Interactions of nocturnal slope flows with ambient winds. Bound.-Layer Meteor, 37, 183-195.

6. Arakawa, A. and V.R. Lamb, 1977: Computational design of the basic dynamical processes of the UCLA general circulation model. Methods of Computational Physics, 17, Academic Press, New York, 173-265.

7. Arya, S.P.S., 1981: Parameterizing the height of the stable atmospheric boundary layer. J. Appl. Meteor., 20, 1192-1202.

8. Asimakopoulos, D., D. Deligiorgi, C Drakopoulos, C. Helmis, K. Kokkori, D. Lalas, K. Sikiotis, and C. Varotsos, 1992: An experimental study of nighttime air pollutant transport over complex terrain in Athens. Atmos. Environ., 26B, 59-71.

9. Atkinson, B.W., 1981: Meso-scale Atmospheric Circulations. Academic Press, New York, $495 \mathrm{pp}$.

10. Atkinson, B.W., 1995: Orographic and stability effects on valley-side drainage flows. Bound.-Layer Meteor., 75, 403-428.

11. Atkinson, B.W. and A.N. Shahub, 1994: Orographic and stability effects on daytime valley-side slope flows. Bound.-Layer Meteor., 68, 275-300.

12. Atmanathan, S., 1931: The katabatic winds of Poona. Indian Meteor. Dept. Sci. Notes, 4, 101-115.

13. Ayer, H., 1961: On the dissipation of drainage wind systems in valleys in morning hours. J. Meteor, 18, 560-563.

14. Bader, D.C., T.B. McKee and G.J. Tripoli, 1992: Mesoscale boundary-layer evolution over complex terrain. Part I: Numerical simulation of the diurnal cycle. J. Atmos. Sci., 44, 2823-2838.

15. Bader, D.C. and T.B. McKee, 1992: Mesoscale boundary-layer evolution over com- 
plex terrain. Par II: Factors controlling nocturnal boundary-layer structure. Mon. Wea. Rev., 120, 802-816.

16. Baines, P.G., 1987: Upstream blocking and airflow over mountains. Ann. Rev. Fluid Mech., 19, 75-97.

17. Ball, F.K., 1956: The theory of strong katabatic winds. Aust. J. Phys., 9, 373-386.

18. Ball, F.K., 1957: The katabatic wind of Adelie Land and King George V Land. Tellus, 9, 201-208.

19. Banta, R.M., 1990: The role of mountain flows in making clouds. Meteorological Monograph, Atmospheric Processes over Complex Terrain, 23, No. 45, Blumen, W. Ed., American Meteorological Society, 229-282.

20. Banta, R.M. and W.R. Cotton, 1981: An analysis of the structure of local wind systems in a broad mountain basin. J. Appl. Meteor, 20, 1255-1266.

21. Banta, R.M. and P.T. Gannon Sr., 1995: Influence of soil moisture on simulations of katabatic flow. Theor. Appl. Climatol., 52, 85-94.

22. Banta, R.M., L.D. Olivier and P.H. Gudiksen, 1993: Sampling requirements for drainage flows that transport atmospheric contaminants in complex terrain. Rad. Protect. Dosim., 50, 243-248.

23. Banta, R.M., L.D. Olivier, and W.D. Neff, 1992: Thermally forced drainage flows in the vicinity of Rocky Flats, Colorado, in winter. Preprints: Sixth Conference on Mountain Meteorology, AMS, Portland, Oregon, 1992, 172-175.

24. Banta, R.M., L.D. Olivier, W.D. Neff, D.H. Levinson and D. Ruffieux, 1995: Influence of canyon-induced flows on flow and dispersion over adjacent plains. Theor. Appl. Climatol., 52, 27-42.

25. Barr, S. and M.M. Orgill, 1989: Influence of external meteorology on nocturnal valley drainage winds. J. Appl. Meteor., 28, 497-517.

26. Barry, R.G., 1992: Mountain Weather and Climate. 2nd Ed., New York, Routledge.

27. Batchelor, G. K., 1953: The conditions for dynamical similarity of motions of a frictionless perfect-gas atmosphere. Quart. J. Roy. Meteor. Soc., 29, 224-235.

28. Baughman, R.G., 1981: Why windspeeds increase on high mountain slopes at night. U.S. Forest Service Research Paper INT-276, 1-6.

29. Baumann, K., E.J. Williams, J.A. Olson, J.W. Harder and F.C. Fehsenfeld, 1996: Meteorological characteristics and spatial extent of upslope events during the 1993 tropospheric $\mathrm{OH}$ photochemistry experiment. J. Geophys. Res. - Atmos. (accepted).

30. Bell, R.C. and R.O.R. Thompson, 1980: Valley ventilation by cross winds. J. Fluid Mech., 96, 757-767.

31. Bjerknes, V., 1902: Zirkulation relativ zu der Erde. Meteor. Z., 19, 97-108.

32. Blackadar A.K., 1957: Boundary layer wind maximum and their significance for the growth of nocturnal inversions. Bull. Amer. Meteor. Soc., 38, 283-290. 
33. Blackadar A.K., 1979: High resolution models of the planetary boundary layer. $A d v$. in Geophys., 50-85.

34. Bleck, R. and S.G. Benjamin, 1993: Regional weather prediction with a model combining terrain-following and isentropic coordinates. Part I: Model description. Mon. Wea. Rev., 121, 1770-1785.

35. Blondin, C., 1984: Meteorology of medium-range transport of pollution. Atmos. Environ., 18, 537-544.

36. Blumen, W., 1984: An observational study of instability and turbulence in nighttime drainage winds. Bound.-Layer Meteor, 28, 245-269.

37. Blumen, W. and C.D. McGregor, 1976: Wave drag by three-dimensional mountain lee waves in nonplanar shear flow. Tellus, 28, 287-298.

38. Bondy, F., 1935: Uber beziehungen zwischen periodischen talwinden und verschiedenen meteorologischen faktoren [Relationships between periodic valley winds and various meteorological factors]. Ph. D. Dissertation, Innsbruck.

39. Bonner, W.D., 1968: Climatology of the low level jet. Mon. Wea. Rev., 96, 833-849.

40. Bossert, J.E. and W.R. Cotton, 1994: Regional-scale flows in mountainous terrain. Part I: Simplified numerical experiments. Mon. Wea. Rev., 122, 1472-1489.

41. Bossert, J.E. and W.R. Cotton, 1994: Regional-scale flows in mountainous terrain. Part II: A numerical and observational comparison. Mon. Wea. Rev., 122, 1449-1471.

42. Bossert, J.E., J.D. Sheaffer and E.R. Reiter, 1989: Aspects of regional-scale flows in mountainous terrain. J. Appl. Meteor, 28, 590-601.

43. Bossert, J.E. and G.S. Poulos, 1995: A numerical investigation of mechanisms affecting drainage flows in highly complex terrain. Theor. Appl. Climatol., 52, 119-134.

44. Brehm, M., 1986: Experimentelle und nuerische untersuchungen der hangwindsschicht und ihrer rolle bei der erwarmung von talern. [Experimental and numerical investigations of the slope wind layer and its role the warming of valleys] Wiss. Mitt. Nr. 54, PhD. dissertation, Universitat Munchen, Meteorologisches Institut, Munchen, FRG, 150 pp.

45. Brehm, M., and C. Freytag, 1982: Erosion of the nighttime thermal circulation in an alpine valley. Arch. Meteor. Geoph. Biokl., B31, 331-352.

46. Bretherton, F.P., 1969: Momentum transport by gravity waves. Quart. J. Roy. Meteor. Soc., 95, 213-243.

47. Briggs, G.A., 1979: Analytic modeling of drainage flows. Draft document. Atmospheric Turbulence and Diffusion Laboratory. NOAA, Oak Ridge, TN, 37830, December.

48. Briggs, G.A., 1981: Canopy effects on predicted drainage flow characteristics and comparisons with observations. Proc. 5th Symp. on Turb. and Diff., Atlanta. Amer. Meteor. Soc., Boston, MA, 113-115.

49. Britter, R.E. and P.F. Linden, 1980: The motion of the front of a gravity current travel- 
ling down an incline. J. Fluid Mech., 99, 531-543.

50. Broder, B., H.U. Dutsch and W. Graber, 1981: Ozone fluxes in the nocturnal planetary boundary layer over hilly terrain. Atmos. Environ., 15, 1195-1199.

51. Broeke, M.R. van den, P.G. Duynkerke and E.A.C. Henneken: 1994: Heat, momentum and moisture budgets of the katabatic layer over the melting zone of the West Greenland ice sheet in summer. Bound.-Layer Meteor., 71, 393-413.

52. Brost, R.A. and J.C. Wyngaard, 1978: A model study of the stably stratified planetary boundary layer. J. Atmos. Sci., 35, 1427-1440.

53. Buettner, K.J.K., 1967: Valley wind, sea breeze, and mass fire: Three cases of quasistationary airflow. Proceedings of the symposium on mountain meteorology, 26 June, Colorado State University, Department of Atmospheric Science, Fort Collins, Colorado, Eds. E.R. Reiter and J.L Rasmussen, Atmospheric Science Paper \#122.

54. Buettner, K.J.K. and N. Thyer, 1959: On valley and mountain winds. final Report, Contract No. AF19(604)-2289, AFCRC-TR-59-283, University of Washington, Department of Meteor. Clim.

55. Buettner, K.J.K. and N. Thyer, 1965: Valley winds in the Mount Rainier area. Arch. Meteor. Geophys. Biokl., 14, H. 2, 125-147.

56. Businger, J.A. and S.P.S. Arya, 1974: Height of the mixed layer in the stably stratified planetary boundary layer. $A d v$. in Geophys, 18A, 73-92.

57. Carruthers, D.J. and J.C.R. Hunt, 1990: Fluid mechanics of airflow over hills: Turbulence, fluxes and waves in the boundary layer. Meteorological Monograph, Atmospheric Processes over Complex Terrain, 23, No. 45, Blumen, W. Ed., American Meteorological Society, 83-107.

58. Castro, I.P., 1987: A note on lee wave structures in stratified flow over three-dimensional obstacles. Tellus, 39A, 72-81.

59. Castro, I. P., W. H. Snyder and G. L. Marsh, 1983: Stratified flow over three-dimensional ridges. J. Fluid Mech., 135, 261-282.

60. Caughey, S.J., 1977: Boundary-layer turbulence spectra in stable conditions. Bound.Layer Meteor, 11, 3-14.

61. Caughey, S.J. and C.J. Readings, 1975: An observation of waves and turbulence in the Earth's boundary layer. Bound.-Layer Meteor., 9, 279-96.

62. Caughey, S.J., J.C. Wyngaard and J.C. Kaimal, 1979: Turbulence in the evolving stable boundary layer. J. Atmos. Sci., 36, 1041-1052

63. Chen, C., 1995: Numerical simulations of gravity currents in uniform shear flows. Mon. Wea. Rev., 123, 3240-3253.

64. Chen, C. and W.R. Cotton, 1983: Numerical experiments with a one-dimensional higher order turbulence model: Simulation of the Wangara Day 33 case. Bound.Layer Meteor, 25, 375-404.

65. Chien, C.W., S. Hozaki and D.P. Mack, 1966: Study of mesoscale flows. MRI66 FR- 
370, Final Report, Meteorology Research, Inc., Altadena, California.

66. Chopra, K.P., 1973: Atmospheric and oceanic flow probelms introduced by islands. Adv. Geophys., 16, 297-421.

67. Chorin, A. J., 1968: Numerical solution of the Navier-Stokes equations. Math. Comp., 22, 745-762.

68. Clark, T.L., 1977: A small-scale dynamic model using a terrain-following coordinate transformation. J. Comput. Phys., 24, 186-215.

69. Clements, W.E., J.A. Archuleta and D.E. Hoard, 1989: Mean structure of the nocturnal drainage flow in a deep valley. J. Appl. Meteor., 28, 457-462.

70. Clements, W.E. and C.J. Nappo, 1983: Observations of a drainage flow event on a high-altitude simple slope. J. Clim. Appl. Meteor, 22, 331-335.

71. Cornfeld, C.E., 1938: Katabatic winds and the prevention of frost damage. Quart. J. Roy. Meteor. Soc., 64, 553-584.

72. Coulter, R.L. and P. Gudiksen, 1995: The dependence of canyon winds and surface cooling and external forcing in Colorado's Front Range. J. Appl. Meteor, 34, 14191429.

73. Coulter, R.L. and T.J. Martin, 1991: Argonne National Laboratory operations during ASCOT 1991. December, Environmental Research Division, Argonne National Laboratory, Argonne, IL, 59 pp.

74. Coulter, R.L., M. Orgill and W. Porch, 1989: Tributary fluxes into Brush Creek Valley. J. Appl. Meteor., 28, 555-568.

75. Cotton, W.R., M.A. Stephens, T. Nehrkorn, and G.J. Tripoli, 1982: The Colorado State University cloud/mesoscale model-1982. Part II: An ice phase parameterization. Contrib. Atmos. Phys., 16, 295-320.

76. Cotton, W.R., C.J. Tremback, and R.L. Walko, 1988: CSU RAMS - A cloud model goes regional. Proc. NCAR Workshop on Limited-Area Modeling Intercomparison, Nov. 15-18, NCAR, Boulder, CO, 202-211.

77. Cotton, W. R. and G. J. Tripoli, 1978: Cumulus convection in shear flow - Threedimensional numerical experiments. J. Atmos. Sci., 35, 1503-1521.

78. Cramer, O.P., 1972: Potential temperature analysis for mountainous terrain. J. Appl. Meteor., 11, 44-50.

79. Cramer, O.P. and R.E. Lynott, 1961: Cross-section analysis in the study of windflow over mountainous terrain. Bull. Amer. Meteor. Soc., 42, 693-702.

80. Crook, N.A., 1986: The effect of ambient stratification and moisture on the motion of atmospheric undular bores. J. Atmos. Sci., 43, 171-181.

81. Crook, N.A., 1988: Trapping of low-level internal gravity waves. J. Atmos. Sci., 45, 1533-1541.

82. Davidson, B., 1961: Valley wind phenomena and air pollution problems. J. Air Pollut. Contr. Assoc., 11, 364-383. 
83. Davidson, B., 1963a: Reflections on some recent observations of local wind systems. Transactions of the New York Academy of Sciences, Series II, 25, 663-673.

84. Davidson, B., 1963b: Some turbulence and wind variability observations in the lee of mountain ridges. J. Appl. Meteor., 2, 463-472.

85. Davidson, B. and P.K. Rao, 1963: Experimental studies of the valley-plain wind. Int. $J$. Air Water Pollut., 7, 907-923.

86. Davies, H.C., 1976: A lateral boundary formulation for multi-level prediction models. Quart. J. Roy. Meteor. Soc., 102, 405-418.

87. Davies, H.C. and R.E. Turner, 1977: Updating prediction models by dynamical relaxation: an examination of the technique. Quart. J. Roy. Meteor. Soc., 103, 225-245.

88. Davies, H.C. and C. Schar, 1986: Diabatic modification of airflow over a mesoscale orographic ridge: A model study of the coupled response. Quart. J. Roy. Meteor. Soc., 112, 711-730.

89. Defant, A., 1933: Der abfluss schwerer luftmassen auf geneigten boden, nebst einigen bemerkungen zur theorie stationarer luftstrome. [The flow of heavy air masses down slopes along with some remarks on the theory of stationary air currents] Sitz. Berichte Preuss. Akad. Wiss. Phys.-Math.-Klasse, 18, 624-635.

90. Defant, F., 1949: Zur theorie der hangwinde, nebst bemerkungen zur theorie der bergund talwinde [A theory of slope winds, along with remarks on the theory of mountain and valley winds]. Archiv fuer Meteorologie Geophysik und Bioklimatologie, Ser. A, 1, 421-450.

91. Defant, F., 1951: Local winds. Compendium of Meteorology, T.F. Malone, Ed., Amer. Meteor. Soc., 655-672.

92. Derbyshire, S.H., 1995a: Stable boundary layers: Observations, models and variability Part I: Modelling and measurements. Bound-Layer Meteor., 74, 19-54.

93. Derbyshire, S.H., 1995b: Stable boundary layers: Observations, models and variability Part II: Data analysis and averaging effects. Bound-Layer Meteor., 75, 1-24.

94. Devara, P.C.S., P.E. Raj, B.S. Murthy, G. Pandithurai, S. Sharma and K.G. Vernekar, 1995: Intercomparison of nocturnal lower-atmospheric structure observed with lidar and sodar techniques at Pune, India. J. Appl. Meteor., 34, 1375-1383.

95. Dias, N.L., W. Brutsaert and M.L. Wesely, 1995: Z-less stratification under stable conditions. Bound.-Layer Meteor., 75, 175-187.

96. Doran, J.C., 1996: The influence of canyon winds on flow fields near Colorado's Front Range. J. Appl. Met, 35, No. 4 (in publication).

97. Doran, J.C., 1991: The effects of ambient winds on valley drainage flows. Bound.Layer Meteor, 55, 177-189.

98. Doran, J.C. and T.W. Horst, 1981: Velocity and temperature oscillations in drainage winds. J. Appl. Meteor, 20, 361-364.

99. Doran, J.C. and T.W. Horst, 1983: Observations and models of simple nocturnal slope 
flows. J. Atmos. Sci., 40, 708-717.

100. Doran, J.C. and E.D. Skyllingstad, 1992: Multiple-scale terrain forcing of local wind fields. Mon. Wea. Rev., 120, 817-825.

101. Doran, J.C., M.L. Wesely, R.T. McMillen and W.D. Neff, 1989: Measurements of turbulent heat and momentum fluxes in a mountain valley. J. Appl. Meteor:, 28, $438-444$.

102. Drazin, P.G., 1961: On the steady flow of a fluid of variable density past an obstacle. Tellus, 13, 239-251.

103. Durran, D.R., 1990: Mountain waves and downslope winds. Meteorological Monograph, Atmospheric Processes over Complex Terrain, 23, No. 45, Blumen, W. Ed., American Meteorological Society, 59-81.

104. Durran, D.R. and J.B. Klemp, 1982: On the effects of moisture on the Brunt-Vaisala frequency. J. Atmos. Sci., 39, 2152-2158.

105. Egan, B.A., 1984: Transport and diffusion in complex terrain (a review). Bound.Layer Meteor, 30, 3-28.

106. Egger, J., 1981: On the linear two-dimensional theory of thermally induced slope winds. Beitr. Phys. Atmos., 54, 465-481.

107. Egger, J., 1990: Thermally forced flows: Theory. Meteorological Monograph, Atmospheric Processes over Complex Terrain, 23, No. 45, Blumen, W. Ed., American Meteorological Society, 43-57.

108. Eidenshink, J.C., 1992: The 1990 Coterminous U.S. AVHRR data set. Photo. Eng. Rem. Sens., 58, 809-813.

109. Einaudi, F. and J.J. Finnigan, 1981: The interaction between an internal gravity wave and the planetary boundary layer. Part I: The linear analysis. Quart. J. Roy. Meteor. Soc., 107, 793-806.

110. Ek, M. and R.H. Cuenca, 1994: Variation in soil parameters: Implications for modeling surface fluxes and atmospheric boundary layer development. Bound. Laver Meteor, , 70, 369-383.

111. Ekhart, E., 1932a: Zur aerologie des berg und talwinds, ergebnisse von pilotballonaufstiegen in Innsbruck. Beitr. Phys. freien Atmos., 18, 1-26.

112. Ekhart, E., 1932b: Weitere beitrage zum problem des berg und talwindes. Beitr. Phys. freien Atmos., 18, 242-252.

113. Ekhart, E., 1934: Neuere untersuchungen zure aerologie der talwinde. Beitr. Phys. d. fr. Atmos., 21, 245-268.

114. Ekhart, E., 1944: Beitrage zur alpinen meteorologie. [Contributions to alpine meteorology], Meteorologische Zeitschrigt, 61, 217-231.

115. Ekhart, E., 1948: De la structure thermique de l'atmosphere dans la montagne [On the thermal structure of the mountain atmosphere]. La Meteorologie, 4, 3-26.

116. Elder, J., 1965: Laminar free convection in a vertical slot. J. Fluid Mech., 23, 77-98. 
117. Eliassen, A, and E. Palm, 1960: On the transfer of energy in stationary mountain waves. Geofys. Publ. Oslo, 22, 1-23.

118. Ellison, T.H. and J.S. Turner, 1959: Turbulent entrainment in stratified flows. J. Fluid Mech., 6, 423-448.

119. Enger, L., D. Koracin and X. Yang, 1993: A numerical study of boundary layer dynamics in a mountain valley. Bound.-Layer Meteor, 66, 357-394.

120. Erasmus, D.A., K.T. Fredin, M.L. Gauthier and S.M. Reiter, 1993: Observations of the nocturnal stable boundary layer in the Greeley area. Preprints, Front Range AGU meeting, Fall, Denver, Colorado.

121. Finnigan, J.J. and F. Einaudi, 1981: The interaction between an internal gravity wave and the planetary boundary layer. Part II: Effect of the wave on the turbulence structure. Quart. J. Roy. Meteor. Soc., 107, 807-832.

122. Finnigan, J.J., F. Einaudi and D. Fua, 1984: The interaction between an internal gravity wave and turbulence in the stably-stratified nocturnal boundary layer. J. Atmos. Sci., 41, 2409-2436.

123. Fitzjarrald, D.R., 1984: Katabatic wind in opposing flow. J. Atmos. Sci., 41, 1143 1158.

124. Fleagle, R.G., 1950: A theory of air drainage. J. Meteor, 7, 227-232.

125. Fosberg, M.A., 1969: Airflow over a heated coastal mountain. J. Appl. Meteor, 8, 436-442.

126. Fournet, M.J., 1840: Des brises de jour et de nuit autour des montagnes. [Daytime and nighttime winds around mountains], Annls. Chim.Phys., 74, 337-401.

127. Fua, D., G. Chimonas, F. Einaudi and O. Zeman, 1982: An analysis of wave-turbulence interaction. J. Atmos. Sci., 39, 2450-2462.

128. Gal-Chen, T. and R. C. J. Somerville, 1975a: On the use of a coordinate transformation for the solution of the Navier-Stokes equations. J. Comput. Phys., 17, 209228.

129. Gal-Chen, T. and R. C. J. Somerville, 1975b: Numerical solution of the NavierStokes equations with topography. J. Comput. Phys., 17, 276-310.

130. Garratt, J.R., 1982: Observations in the nocturnal boundary layer. Bound-Layer Meteor, 22, 21-48.

131. Garratt, J.R. and R.A. Brost, 1982: Radiative cooling effects within and above the nocturnal boundary layer. J. Atmos. Sci., 38, 2730-2746.

132. Gary, J. M., 1973: Estimate of truncation error in transformed coordinate, primitive equation atmospheric models. J. Atmos. Sci., 30, 223-233.

133. Geiger, R., 1975: The climate near the ground. Harvard Univ. Press, Cambridge, Massachusetts, 5th printing, $611 \mathrm{pp}$.

134. Gifford, F.A., 1982: Horizontal diffusion in the atmosphere: A Lagrangian-dynamical theory. Atmos. Environ., 16, 505-512. 
135. Gill, A.E., 1966: The boundary layer regime for convection in a rectangular cavity. $J$. Fluid Mech., 26, 515-536.

136. Gill, A.E., 1982: Studies of moisture effects in simple atmospheric models: The stable case. Geophys. Astrophys. Fluid Dynamics, 19, 119-152.

137. Glatzmaier, T.G, W.E. Clements and W.L. Spurgeon, 1991: Summary and data of the Los Alamos measurement program during the 1991 ASCOT field study. LA-UR91-2457, ASCOT-91-2, Los Alamos National Laboratory, Los Alamos, NM $87545,113 \mathrm{pp}$.

138. Gleeson, T.A., 1953: Effects of various factors on valley winds., J. Meteor., 10, 262269.

139. Grant, A.L.M. and P.J. Mason, 1990: Observations of boundary layer structure over complex terrain. Quart. J. Roy. Meteor. Soc., 116, 159-86.

140. Gudiksen, P.H., 1989: Categorization of nocturnal drainage flows within the Brush Creek Valley and the variability of $\sigma_{\theta}$ in complex terrain. J. Appl. Meteor, 28, 489495.

141. Gudiksen, P.H., J.M. Leone, C.W. King, D. Ruffieux and W.D. Neff, 1992: Measurements and modeling of the effects of ambient meteorology on nocturnal drainage flows. J. Appl. Meteor., 31, 1023-1032.

142. Gutman, L.N., 1953: On slope winds over a surface of small slope. Bull. Acad. Sci. USSR, Ser. Geogr., 4, 377-379.

143. Gutman, L.N., 1972: Introduction to the non-linear theory of mesoscale meteorological processes. Israel Program for Scientific Translation, Jerusalem, $274 \mathrm{pp}$.

144. Gutman, L.N., 1983: On the theory of the katabatic slope wind. Tellus, 35A, 213218.

145. Hallett, J., 1972: Breaking waves at an inversion. Mon. Wea. Rev., 100, 103-105.

146. Hann, J. von, 1879: Zur meteorologie der alpengifel. Wien Akad. Wiss. Sitzungsherichte (Math.-Naturwiss. Klass.), 78, 829-866.

147. Harlow, F. H. and J. E. Welch, 1965: Numerical calculation of time-dependent viscous incompressible flow of fluid with free surface. Phys. Fluids, 8, 2182-2189.

148. Heilman, W. and R. Dobosy, 1985: A nocturnal atmospheric drainage flow simulation investigating the application of one-dimensional modeling and current turbulence schemes., J. Clim. Appl. Meteor, 24, 924-936.

149. Hennemuth, B. and H. Schmidt, 1985: Wind phenomena in the Dischma Valley during DISKUS. Arch. Meteor. Geoph. Biokl., Ser. B, 35, 361-387.

150. Heywood, G.S.P., 1933: Katabatic winds in a valley. Quart. J. Roy. Meteor. Soc., 59, 47-58.

151. Hines, C.O., 1972: Gravity waves in the atmosphere. Nature, 239, 73-78.

152. Hoinkes, H., 1954: Beitrage zur kenntnis des gletscherwindes. Arch.f. Meteor., B6, 36-53. 
153. Holton, J.R., 1967: The diurnal boundary layer wind oscillation above sloping terrain. Tellus, 19, 199-205.

154. Hootman, B.W. and W. Blumen, 1983: Analysis of nighttime drainage winds in Boulder, Colorado during 1980. Mon. Wea. Rev., 111, 1052-1061.

155. Horn, J.D. and E.J. Trawle, 1964: Orographic effects on wind variability. U.S. Army Electronics Research and Development Activity, White Sands Missile Range, New Mexico, ERDS-157, 20 pp.

156. Horst, T.W. and J.C. Doran, 1986: Nocturnal drainage flow on simple slopes. Bound.-Layer Meteor, 3, 263-286.

157. Horst, T.W. and J.C. Doran, 1988: The turbulence structure of nocturnal slope flow. J. Atmos. Sci., 45, 605-616.

158. Hunt, J. C. R., and W. H. Snyder., 1980: Experiments on stably and neutrally stratified flow over a model three-dimensional hill. J. Fluid Mech., 96, 671-704.

159. Jaffe, S., 1958: Effect of prevailing wind on the valley wind regime at anemometer level, preliminary report on valley wind studies in Vermont. AFCRC-TR-58-29, Air Force Research Cambridge Laboratory, $32 \mathrm{pp}$.

160. Janjic, T. Z. and Z. I. Janjic, 1993: Spectral distributions of the pressure gradient force errors in $\sigma$-coordinate spectral and grid-point models in an idealized case. Meteor. Atmos. Phys. 52, 129-135.

161. Janjic, Z. I., 1977: Pressure gradient force and advection scheme used for forecasting with steep and small scale topography. Contrib. Atmos. Phys., 50, 186-199.

162. Janjic, Z. I., 1989: On the pressure gradient force error in $\sigma$-coordinate spectral models. Mon. Wea. Rev, 117, 2285-2292.

163. Jeffreys, H., 1922: On the dynamics of wind. Quart. J. Roy. Meteor. Soc., 48, 29-48.

164. Jeffreys, H., 1925: Phil. Mag., 49, 793.

165. Kaimal, J.C., 1973: Turbulence spectra, length scales and structure parameters in the stable surface layer. Bound.-Layer Meteor., 4, 289-309.

166. Kanitscheider, R., 1936: Beitraege zur mechanik des foehns [Contributions to the mechanics of the foehn]. Beitr. Physik fr. Atmos., 18, 27-35.

167. Kasahara, A., 1974: Various vertical coordinate systems used for numerical weather prediction. Mon. Wea. Rev, 102, 513-522.

168. Kelvin, Lord, 1866: On stationary waves in flowing water. Philos. Mag., 5, 22, 353$357,445-452,517-530$.

169. Kim, J. and L. Mahrt, 1992: Simple formulation of turbulent mixing in the stable free atmosphere and nocturnal boundary layer. Tellus, 44A, 381-394.

170. Kitabayashi, K., 1977: Wind tunnel and field studies of stagnant flow upstream of a ridge. $J$. Meteor. Soc. Japan, 55, 193-203.

171. Klemp, J. B. and D. R. Durran, 1983: An upper boundary condition permitting inter- 
nal gravity wave radiation in numerical mesoscale models. Mon. Wea. Rev., 111, 430-444.

172. Klemp, J. B., and D. K. Lilly, 1978: Numerical simulation of hydrostatic mountain waves. J. Atmos. Sci., 35, 78-107.

173. Klemp, J.B. and R.B. Wilhelmson, 1978a: The simulation of three-dimensional convective storm dynamics. J. Atmos. Sci., 35, 1070-1096.

174. Kondo, J., O. Kanechika and N. Yasuda, 1978: Heat and momentum transfers under strong stability $n$ the atmospheric surface layer. J. Atmos. Sci., 35, 1012-1021.

175. Kondo, H., 1984: The difference of the slope wind between day and night. J. Meteor: Soc. Japan, 62, 224-233.

176. Koop, C.G. and F.K. Browand, 1979: Instability and turbulence in a stratified fluid with shear. J. Fluid Mech., 93, 135-159.

177. Koschmieder, H., 1921: Zwei bemerkenswerte beispiele horizontaler wolkenschlauche. Beitr. Phys. frei. Atmos., 9, 176-180.

178. Kozhevnikov, V.N., and A.P. Pavlenko, 1993: Atmospheric disturbances over mountains and flight safety. Phys. Atmos. Ocean, English translation, 29, 283-295.

179. Kraichnan, R. H., 1976: Eddy viscosity in two and three dimensions. J. Atmos. Sci., 33, 1521-1536.

180. Kuettner, J. 1959: The rotor flow in the lee of mountains. G. R.D. Res. Notes, No. 6, Air Force Cambridge Research Center, $20 \mathrm{pp}$.

181. Kuettner, J., 1968: Lee waves in the Colorado Rockies. Weatherwise, 21, 180-195.

182. Kurihara, Y., 1968: Note on finite-difference expressions for the hydrostatic relation and pressure gradient force. Mon. Wea. Rev, 96, 654-656.

183. Kurzeja, R.J., S. Berman and A.H. Weber, 1991: A climatological study of the nocturnal planetary boundary layer. Bound.-Layer Meteor, 54, 105-128.

184. Kyle, T.G., 1987: The energy budget in a valley nocturnal flow. Tellus, 39A, 226234.

185. Lalas, D.P. and.F. Einuadi, 1973: On the stability of a moist atmosphere in the presence of a background wind. J. Atmos. Sci., 30, 795-800.

186. Lapidus, A. 1967: A detached shock calculation by second-order differences. $J$. Comput. Phys., 2, 154-177.

187. Leahey, D.M., 1974: A study of airflow over irregular terrain. Atmos. Environ., 8, 783-791.

188. Lee, J.T., R.E. Lawson Jr. and G.L. Marsh, 1987: Flow visualization experiments on stably stratified flow over ridges and valleys. Meteor. Atmos. Phys., 37, 183-194.

189. Lee, T.J., R.A. Pielke, R.C. Kessler and J. Weaver, 1989: Influence of cold pools downstream of mountain barriers on downslope winds and flushing. Mon. Wea. Rev., 117, 2041-2058. 
190. Lenschow, D.H., S.F. Zhang and B.B. Stankov, 1988: The stably stratified boundary layer over the Great Plains II. Horizontal variations and spectra. Bound.-Laver Meteor., 42, 123-135.

191. Lester, P.F. and W.A. Fingerhut, 1973: Lower turbulent zones associated with mountain lee waves. J. Appl. Meteor, 13, 54-61.

192. Lettau, H.H., 1966: A case study of katabatic flow on the South Polar plateau. Studies in Antarctic Meteorology, M.J. Rubin, Ed., American Geophysical Union, Antarctic Res. Ser, 9, 1-11.

193. Lettau, H.H., 1967: Small to large-scale features of boundary layer structure over mountain slopes. Proceedings of the symposium on mountain meteorology, 26 June, Colorado State University, Department of Atmospheric Science, Fort Collins, Colorado, Eds. E.R. Reiter and J.L Rasmussen, Atmospheric Science Paper $\# 122$.

194. Leyi, Z. and H.A. Panofsky, 1983: Wind fluctuations in stable air at the Boulder tower. Bound.-Layer Meteor, 25, 353-362.

195. Lilly, D. K., 1978: A severe downslope windstorm and aircraft turbulence event induced by a mountain wave. J. Atmos. Sci., 35, 59-77.

196. Loewe, F., 1935: Das klima des Gronlandischen inlandeises. Handbuch der Klimatologie, $2(\mathrm{~K}), 67-101$.

197. Long, R.R., 1975: The influence of shear on mixing across density interfaces. $J$. Fluid. Mech., 70, 305-320.

198. Luhar, A.K. and K.S. Rao, 1993: Random-walk model studies of the transport and diffusion of pollutants in katabatic flows. Bound-Layer Meteor, 66, 395-412.

199. Lykosov, V.N. and L.N. Gutman, 1972: Turbulent boundary layer above a sloping underlying surface. Izv. Atmos. Oceanic Phys., 8, 462-467.

200. Lyra, G., 1943: Theorie der stationaren leewellenstromung in freier atmosphare, Zeitschr: Angew. Math. Mech., 23, 1-18.

201. Macomber. E.M. and J.O. Ledbetter, 1975: A drainage wind flow model. Environ. Let., 10, 177-190.

202. Mahrer, Y., 1984: An improved numerical approximation of the horizontal gradients in a terrain-following coordinate system. Mon. Wea. Rev., 112, 918-922.

203. Mahrer, Y. and R.A. Pielke, 1977a: A numerical study of the airflow over irregular terrain. Contr: Phys. Atmos., 50, 98-113.

204. Mahrer, Y. and R.A. Pielke, 1977b: The effects of topography on sea and land breezes in a two-dimensional numerical model. Mon. Wea. Rev., 105, 1151-1162.

205. Mahrt, L., 1982: Momentum balance of gravity flows. J. Atmos. Sci., 39, 2701-2711.

206. Mahrt, L., R.C. Heald, D.H. Lenschow, B.B. Stankov and I. Troen, 1979: An observational study of the structure of the nocturnal boundary layer. Bound.-Layer Meteor, 17, 247-264. 
207. Mahrt, L., 1985: Vertical structure and turbulence in the very stable boundary layer. J. Atmos. Sci., 42, 2333-2349.

208. Mahrt, L. and N. Gamage, 1987: Observations of turbulence in stratified flow. $J$. Atmos. Sci., 44, 1106-1121.

209. Mahrt, L. and S. Larsen, 1990: Relation of slope winds to the ambient flow over gentle terrain. Bound.-Layer Meteor, 53, 93-102.

210. Malm, W.C., K.A. Gebhart, D. Latimer, T. Cahill, R. Eldred, R.A. Pielke, R.A. Stocker, and J. Watson, 1989: Winter Haze Intensive Tracer EXperiment (WHITEX), Prepared for the U.S. Department of the Interior, National Park Service.

211. Manins, P.C., 1992: Vertical fluxes in katabatic flows. Bound-Layer Meteor, 60, 169-178.

212. Manins, P.C. and B.L. Sawford, 1979a: A model of katabatic winds. J. Atmos. Sci., 36, 619-630.

213. Manins, P.C. and B.L. Sawford, 1979b: Katabatic winds: A field case study. Quart. J. Roy. Meteor. Soc., 105, 1011-1025.

214. Mason, P.J., 1987: Diurnal variations in flow over a succession of ridges and valleys. Quart. J. Roy. Meteor. Soc., 113, 1117-1140.

215. Mawson, D., 1915: Home of the Blizzard. I, Heineman, London.

216. Mayr, G.J., 1993: The evolution of orogenic blocking. Ph.D. dissertation, Colorado State University, Department of Atmospheric Science, Paper\# 534.

217. McCumber, M.C. and R.A. Pielke, 1981: Simulation of the effects of surface fluxes of heat and moisture in a mesoscale model - Part 1. Soil layer. J. Geophys. Res., 86, 9929-9938.

218. McElroy, J.L., 1987: Estimation of pollutant transport and concentration distributions over complex terrain of southern California using airborne lidar. J. Air Pollut. Control Assoc., 37, 1046-1051.

219. McHattie, L.B., 1968: Kananaskis Valley winds in summer. J. Appl. Meteor, 7, 348352.

220. McKee, T.B and R.D. O'Neal, 1989: The role of valley geometry and energy budget in the formation of nocturnal valley winds. J. Appl. Meteor., 28, 445-456.

221. McNider, R.T., 1981: Investigation of the impact of topographic circulations on the transport and dispersion of air pollutants. Ph.D. Dissertation, University of Virginia.

222. McNider, R.T., 1982: A note on velocity fluctuations in drainage flows. J. Atmos. Sci., 39, 1658-1660.

223. McNider, R.T., M.D. Moran, and R.A. Pielke, 1988: Influence of diurnal and inertial boundary-layer oscillations on long-range dispersion. Atmos. Environ., 22, $2445-$ 2462.

224. McNider, R.T. and R.A. Pielke, 1981: Diurnal boundary-layer development over 
sloping terrain. J. Atmos. Sci., 38, 2198-2212.

225. McNider, R.T. and R.A. Pielke, 1984: Numerical simulation of slope and mountain flows. J. Clim. Appl. Meteor, 23, 1441-1453.

226. Mellor, G.L. and T. Yamada, 1974: A hierarchy of turbulence closure models for planetary boundary layers. J. Atmos. Sci., 31, 1791-1806.

227. Mendonca, B.G., 1969: Local wind circulation on the slopes of Mauna Loa. J. Appl. Meteor, 8, 533-541.

228. Meroney, R.N., 1990: Fluid dynamics of flow over hills/mountains-Insights obtained through physical modeling. Meteorological Monograph. Atmospheric Processes over Complex Terrain, 23, No. 45, Blumen, W. Ed., American Meteorological Society, 145-171.

229. Mesinger, F., 1982: On the convergence and error problems of the calculation of the pressure gradient force in $\sigma$ coordinate models. Geophys. Astrophys. Fluid Dynamics, 19, 105-117.

230. Mihailovic, D. T. and Z. I. Janjic, 1986: Comparison of methods for reducing the error of the pressure gradient force in $\sigma$ coordinate models. Meteor. Atmos. Phys., 35, 177-184.

231. Miranda, P. M. A., and I. N. James, 1992: Non-linear three-dimensional effects on gravity-wave drag: Splitting flow and breaking waves. Quart. J. Roy. Meteor. Soc., $118,1057-1081$.

232. Mitchell, M.K. and R.A. Arritt, 1995: An hourly climatology of the summertime Great Plains low-level jet using wind profiler observations. Weather and Forecasting, 10, 576-591.

233. Moran, M.D., 1992: Numerical modeling of mesoscale atmospheric dispersion. Ph.D. Dissertation, Colorado State University, Department of Atmospheric Science, Paper No. 513, Fort Collins, Colorado, 80523, 758 pp.

234. Moriarty, W.W., 1984: The effects of synoptic pressure gradients on drainage winds. International Conference on Mesoscale Meteorology, Melbourne, Australia, Bureau of Meteorology, 35-41.

235. Mursch-Radlgruber, E., 1995: Observations of flow structure in a small forested valley system. Theor. Appl. Climatol., 52, 3-17.

236. Nai-ping, L., W.D. Neff and J.C. Kaimal, 1983: Wave and turbulence structure in a disturbed nocturnal inversion. Bound.-Layer Meteor., 26, 141-155.

237. Nansen, F., 1890: Paa ski over Gronland. Kristiania, Oslo.

238. Nappo, C.J., 1977: Mesoscale flow over complex terrain during the Eastern Tennessee trajectory experiment (ETTEX). J. Appl. Meteor, 16, 1186-96.

239. Nappo, C.J., 1991: Sporadic breakdowns of stability in the PBL over simple and complex terrain. Bound.-Layer Meteor, 54, 69-87.

240. Nappo, C.J. and K.S. Rao, 1987: A model study of pure katabatic flows. Tellus, 39A, 
$61-71$

241. Narimousa, S. R.R. Long and S.A. Kitaigorodskii, 1986: Entrainment due to turbulent shear flow at the interface of a stably stratified fluid. Tellus, 38A, 76-87.

242. Neff, W.D., 1990: Remote sensing of atmospheric processes over complex terrain. Meteorological Monograph, Atmospheric Processes over Complex Terrain, 23, No. 45, Blumen, W. Ed., American Meteorological Society, 173-228.

243. Neff, W.D. and C.W. King, 1987: Observations of complex terrain flows using acoustic sounders: Experiments, topography and winds. Bound.-Layer Meteor. 40 , 363-392.

244. Neff, W.D. and C.W. King, 1988: The accumulation and pooling of drainage flows in a large basin. J. Appl. Meteor, 28, 518-529.

245. Neff, W.D. and C.W. King, 1989: Observations of complex terrain flows using acoustic sounders: drainage flow structure and evolution. Bound.-Layer Meteor, $43,15-41$.

246. Niemeier, U., and K. H. Schlunzen, 1993: Modeling steep terrain influences on flow patterns at the Isle of Helgoland. Contrib. Atmos. Phys., 66, 45-62.

247. Newnham, E.V., 1918: Notes on examples of katabatic wind in the valley of the Upper Thames ath the Aerological Observatory of the Meteorological Office at Benson, Oxon., Professional Notes of the Meteorological Office, London, 1, No. 2 , 14-19.

248. Nicholls, M.E., R.A. Pielke, J.L. Eastman, C.A. Finley, W.A. Lyons, C.J. Tremback, R.L. Walko, and W.R. Cotton, 1995: Applications of the RAMS numerical model to dispersion over urban areas. In: Wind Climate in Cities, J.E. Cermak et al., Editors. Kluwer Academic Publishers, The Netherlands, 703-732

249. Ogura, Y. and N. A. Phillips, 1962: Scale analysis of deep and shallow convection in the atmosphere. J. Atmos. Sci., 19, 173-179.

250. Olson, J.A., K. Baumann, C.J. Volpe, J. Harder, E.J. Williams and G.H. Mount, 1996: Meteorological overview of the $1993 \mathrm{OH}$ photochemistry experiment. $J$. Geophys. Res. - Atmos. (accepted).

251. Orgill, M.M., 1989: Early morning ventilation of a gaseous tracer from a mountain valley. J. Appl. Meteor, 28, 636-651.

252. Orgill, M.M., J.D. Kincheloe and R.A. Sutherland, 1992: Mesoscale influences on nocturnal valley drainage winds in Western Colorado valleys. J. Appl. Meteor, 31, 121-141.

253. Orgill, M.M. and R.I. Schreck, 1985: An overview of the ASCOT multi-laboratory field experiments in relation to drainage winds and ambient flow. Bull. Amer. Meteor. Soc., 66, 1263-1277.

254. Orgill, M.M., R.I. Schreck and C.D. Whiteman, 1981: Synoptically driven downslope winds and their effects on local nocturnal drainage air flow in the Geysers geothermal resource area. Preprints, Second Conference on Mountain Meteorol- 
ogy, Nov 9-12.

255. Orgill, M.M., J.M. Thorp and R. Coulter, 1985: Interaction of sub-mesoscale flows in complex terrain during nocturnal drainage conditions. Proc. 7th Conference on Turbulence and Diffusion, Boulder, CO, Amer. Meteor. Soc., 244-247.

256. Orlanski, I., 1976: A simple boundary condition for unbounded hyperbolic flows. $J$. Comput. Phys., 21, 251-269.

257. Orville, H.D., 1964: On mountain upslope winds. J. Atmos. Sci., 21, 622-633.

258. Pan, Z., S.G. Benjamin, J.M. Brown and T. Smirnova, 1994: Comparative experiments with MAPS on different parameterization schemes for surface moisture flux and boundary-layer processes. Mon. Wea. Rev, 122, 449-470.

259. Panofsky, H.A. and I. van der Hoven, 1955: Spectra and cross-spectra of velocity components in the mesometeorological range. Quart. J. Roy. Meteor. Soc., 81, 603-606.

260. Parker, M.J. and S. Raman, 1993: A case study of the nocturnal boundary layer over a complex terrain. Bound.-Layer Meteor, 66, 303-324.

261. Pellerin, P., R. Laprise and I. Zawadzki, 1995: The performance of a semi-lagrangian transport scheme for the advection-condensation problem. Mon. Wea. Rev, 123, 3318-3330.

262. Perkin, R.G. and Lewis, E.L., 1978: Mixing in an arctic fjord. J. Phys. Oceanogr., 8, 873-880.

263. Perry, S.G., J.M. Norman, H.A. Panofsky and J.D. Martsolf, 1978: Horizontal coherence decay near large mesoscale variations in topography. J. Atmos. Sci., 35, 18841889.

264. Petkovsek, Z., and A. Hocevar, 1971: Night drainage winds. Arch. Meteor: Geophys. Bioklim., A20, 353-360.

265. Petkovsek, Z., 1978: Relief meteorologically relevant characteristics of basins. $Z$. Meteor, 28, 333-340.

266. Petkovsek, Z., 1980: Additional relief meteorologically relevant characteristics of basins. Z. Meteor, 30, 379-382.

267. Petkovsek, Z., 1992: Turbulent dissipation of cold air lake in a basin. Meteor. Atmos. Phys., 47, 237-245.

268. Phillips, N. A., 1957: A coordinate system having some special advantages for numerical forecasting. J. Meteor. (now J. Atmos. Sci.), 14, 184-185.

269. Pielke, R.A., 1974: A three-dimensional numerical model of the sea breezes over south Florida. Mon. Wea. Rev., 102, 115-134.

270. Pielke, R.A., 1984: Mesoscale Meteorological Modeling. Academic Press, New York, N.Y., 612 pp.

271. Pielke, R.A., 1985: The use of mesoscale numerical models to assess wind distribution and boundary layer structure in complex terrain. Bound.-Layer Meteor, 31, 
217-231.

272. Pielke, R. A. and J. Cram, 1989: A terrain-following coordinate system - Derivation of diagnostic relationships. Meteor. Atmos. Phys., 40, 189-193.

273. Pielke, R.A., W.R. Cotton, R.L. Walko, C.J. Tremback, W.A. Lyons, L.D. Grasso, M.E. Nicholls, M.D. Moran, D.A. Wesley, T.J. Lee, and J.H. Copeland, 1992: A comprehensive meteorological modeling system - RAMS. Meteor: Atmos. Phys., 49, 69-91.

274. Pielke, R.A., J.H. Rodriguez, J.L. Eastman, R.L. Walko and R.A. Stocker, 1993: Influence of albedo variability in complex terrain on mesoscale systems. $J$. Climate, 6, 1798-1806.

275. Pielke, R.A. and M. Uliasz, 1992: Influence of landscape variability on atmospheric dispersion. J. Air and Waste Management, 43, 989-994.

276. Pierrehumbert, R.T. and B. Wyman, 1985: Upstream effects of mesoscale mountains. J. Atmos. Sci., 42, 977-1003.

277. Pollak, L.W., 1924: Berg-und Talwinde im Becken von Trient. [Mountain and valley winds in Trento basin] Meteor. Z. 41, 18-21.

278. Porch, W.M., R.B. Fritz, R.L. Coulter and P.H. Gudiksen, 1989: Tributary, valley and sidewall air flow interactions in a deep valley. J. Appl. Meteor, 28, 578-589.

279. Porch, W.M., W.E. Clements, and T.A. Grant, 1990: Lake Powell Basin airflow study in winter. Transactions: Visibility and Fine Particles, October, 1989, Estes Park, Colorado, C.V. Mathai, Ed., Air and Waste Management Association, Pittsburgh, Pennsylvania, 533-540.

280. Post, M.J. and W.D. Neff, 1986: Doppler LIDAR measurements of winds in a narrow mountain valley. Bull. Amer. Meteor. Soc., 67, 274-281.

281. Poulos, G.S., 1992: The potential effect of Los Angeles Basin pollution on Grand Canyon air quality. Atmospheric Science Paper No. 482, Department of Atmospheric Science, Colorado State University, Fort Collins, Colorado 80523, 154 pp.

282. Poulos, G.S., 1994: Observational evidence of mesoscale flows influencing nocturnal boundary layer evolution in complex terrain. American Meteorological Society Sixth Conference on Mesoscale Processes, Portland, Oregon, 18-22 July, J171$\mathrm{J} 174$.

283. Poulos, G.S. and J.E. Bossert, 1995: An observational and prognostic numerical investigation of complex terrain dispersion. J. Appl. Meteor, 34, 650-669.

284. Prandtl, L., 1942: Fuhrer durch die Sromungslehre. Braunschweig, Viewig und Sohn, 367-375.

285. Prandtl, L., 1952: Essentials of fluid dynamics. Hafner, New York, 452 pp., 422-425.

286. Queney, P., 1948: The problem of air flow over mountains: A summary of theoretical studies. Bull. Amer. Meteor. Soc., 29, 16-26.

287. Queney, P., G.A. Corby, N. Gerbier, H. Koschmieder and J. Zierep, 1960: The air- 
flow over mountains. Technical note \#34, M.A. Alaka, Ed., World Meteorological Organization, Geneva, Switzerland.

288. Rao, P.K., 1960: Theoretical investigation of the change of wind speed along the axis of the valley. Progress Report \#5, Contract No. DA-36-039-sc-78127, U.S. Army Signal Research and Development Lab, College of Engineering, New York University.

289. Rao, K.S. and H.F. Snodgrass, 1981: A nonstationary nocturnal drainage flow model. Bound.-Layer Meteor., 20, 309-320.

290. Rasmussen, R., M. Politovich, J. Marwitz, W. Sand, J. McGinley, J. Smart, R. Pielke, S. Rutledge, D. Wesley, G. Stossmeister, B. Bernstein, K. Elmore, N. Powell, E. Westwater, B. B. Stankov and D. Burrows, 1992: Winter Icing and Storms Project (WISP), Bull. Amer. Meteor. Soc., 73, 951-974.

291. Rayleigh, R.J., 1883: The form of standing waves on the surface of running water. Proc. London. Math. Soc., 15, 69-78.

292. Raymond, D.J., 1972: Calculation of airflow over an arbitrary ridge including diabatic heating and cooling. J. Atmos. Sci., 29, 837-843.

293. Rees, J.M., 1991: On the characteristics of eddies in the stable atmospheric boundary layer. Bound.-Layer Meteor, 55, 325-343.

294. Reisner, J.M. and P.K. Smolarkiewicz, 1994: Thermally forced low Froude number flow past three-dimensional obstacles. J. Atmos. Sci., 51, 117-133.

295. Reiter, R., H. Muller, R. Sladkovic and K. Munzert, 1983: Aerologische untersuchungen der tagesperiodischen gebirgswinde unter besonderer berucksichtigung des windfeldẹs im talquerschnitt (Aerological soundings of the mountain-valley wind system with special emphasis on the flow cross-sections through the valley), Meteor. Rdsch., 36, 225-242.

296. Revelle, D.O., 1993: Chaos and 'bursting' in the planetary boundary layer. J. Appl. Meteor, 32, 1169-1180.

297. Richards, L.W., C. Blanchard and D. Blumenthal, 1991: Navajo Generating Station Visibility Study. Final Report, Environmental Services Dept., Salt River Project, Phoenix, Arizona, 85072.

298. Richardson, L.F., 1920: The supply of energy from and to atmospheric eddies. Proc. Roy. Soc. A, 97, 354-373.

299. Romero, R., S. Alonso, E.C. Nickerson and C. Ramis, 1995: The influence of vegetation on the development and structure of mountain waves. J. Appl. Meteor., 34, 2230-2242.

300. Rooney, D. M. and G. S. Janowitz, 1979: Flow over the Rocky and Andes Mountains: Application of an analytical model. J. Atmos. Sci., 36, 549-558.

301. Rotunno, R., and P. K. Smolarkiewicz, 1992: Further results on lee vortices in lowFroude-number flow. J. Atmos. Sci., 48, 2204-2211.

302. Ruscher, P. and L. Mahrt, 1989: Coherent structures in the very stable atmospheric 
boundary layer. Bound.-Layer Meteor, 47, 41-54.

303. Saigey, 1842: Petite Physique de Globe, Paris, Part I.

304. Schumann, U., T. Hauf, H. Holler, H. Schmidt and H. Volkert, 1987: A mesoscale model for the simulation of turbulence, clouds and flow over mountains: Formulation and validation examples. Beitr. Phys. Atmos., 60, 413-446.

305. Scorer, R.S., 1949: Theory of waves in the lee of mountains. Quart. J. Roy. Meteor. Soc, $75,41-56$.

306. Scorer, R.S., 1953: Theory of airflow over mountains: II - The flow over a ridge. Quart. J. Roy. Meteor. Soc., 79, 70-82.

307. Scorer, R.S., 1955: The theory of airflow over mountains - IV. Separation of flow from the surface. Quart. J. Roy. Meteor. Soc., 81, 340-350.

308. Scorer, R.S., 1967: Causes and consequences of standing waves. Proceedings of the symposium on mountain meteorology, 26 June, Colorado State University, Department of Atmospheric Science, Fort Collins, Colorado, Eds. E.R. Reiter and J.L Rasmussen, Atmospheric Science Paper \#122.

309. Scorer, R. S., 1968: Air Pollution. Pergamon Press, Oxford, England, pp-123.

310. Scorer, R.S., 1972: Clouds of the World. Stackpole Books, Harrisburg, Pennsylvania, USA, $254 \mathrm{pp}$.

311. Segal, M., J.R. Garratt, R.A. Pielke, P. Hildebrand, F.A. Rogers, and J. Cramer, 1991: On the impact of snow cover on daytime pollution dispersion. Atmos. Environ., 25B, 177-192.

312. Segal, M., J.F.W. Purdom, J.L. Song, R.A. Pielke and Y. Mahrer, 1986: Evaluation of cloud shading effects on the generation and modification of mesoscale circulations. Mon. Wea. Rev., 114, 1201-1211.

313. Shearer, D.L., 1991: Summary of Field Test Operations for Rocky Flats TRAC Winter Validation Study Tests. October, TRC Environmental Consultants, Inc., Windsor, Connecticut.

314. Shieh, L.J., 1971: An investigation of the morning dissipation of the valley wind by means of a one-dimensional model. Bound.-Layer Meteor., 2, 38-51.

315. Shinn, J.H., R.T. Cederwall, F.J. Gouveia and K.R. Chapman, 1989: Micrometeorology of slope flows in a tributary canyon during the 1984 ASCOT experiment. $J$. Appl. Meteor., 28, 569-577.

316. Sheppard, P.A., 1956: Airflow over mountains. Quart. J. Roy. Meteor. Soc., 82, 528529.

317. Skamarock, W. C. and J. B. Klemp, 1992: The stability of time-split numerical methods for the hydrostatic and the nonhydrostatic elastic equations. Mon. Wea. Rev., 120, 2109-2127.

318. Slade, D.H., 1969: Low turbulence flow in the planetary boundary layer and its relation to certain air pollution problems. J. Appl. Meteor, , 8, 514-522. 
319. Smagorinsky, J., 1963: General circulation experiments with the primitive equations I. The basic experiment. Mon. Wea. Rev., 91, 99-152.

320. Smith, R.B., 1979: The influence of mountains on the atmosphere. Adv. Geophys., 21, 87-230.

321. Smith, R.B., 1990: Why can't stably stratified air rise over high ground?. Meteorological Monograph, Atmospheric Processes over Complex Terrain, 23, No. 45, Blumen, W. Ed., American Meteorological Society, 105-107.

322. Smolarkiewicz, P. K., 1984: A fully multidimensional positive definite advection transport algorithm with small implicit diffusion. J. Comput. Phys., 54, 325-362.

323. Smolarkiewicz, P. K., and T. L. Clark, 1986: The multidimensional positive definite advection transport algorithm. Further development and applications. $J$. Comput. Phys., 67, 396-438.

324. Smolarkiewicz, P. K., and R. Rotunno, 1989: Low Froude number flow past three dimensional obstacles. Part I: Baroclinically generated lee vortices. J. Atmos. Sci. 46, 1154-1164.

325. Smolarkiewicz, P. K., and R. Rotunno, 1990: Low Froude number flow past three dimensional obstacles. Part II: Upwind flow reversal zone. J. Atmos. Sci., 47, 1498-1511.

326. Snook, J.S. and J.M. Schmidt, 1992: A numerical and observational investigation of the 7 January 1992 Denver, Colorado blizzard: Local-scale perspective. Preprints, Sixth Conference on Mountain Meteorology, Portland, Oregon, American Meteorological Society, 101-105.

327. Snyder, W.H., 1985: Fluid modeling of pollutant transport and diffusion in stably stratified flows over complex terrain. Ann. Rev. Fluid Mech., 17, 239-266.

328. Start, G.E., C.R. Dickson and L.L. Wendell, 1975: Diffusion in a canyon within rough mountainous terrain. J. Appl. Meteor., 14, 333-346.

329. Stein, U. and P. Alpert, 1993: Factor separation in numerical simulations. J. Atmos. Sci., 50, 2107-2115.

330. Stigebrandt, A. 1976: Vertical diffusion driven by internal waves in a sill fjord. $J$. Phys. Ocenaogr., 6, 486-495.

331. Stone, G.L. and D.E. Hoard, 1989: Low-frequency velocity and temperature fluctuations in katabatic valley flows. J. Appl. Meteor., 28, 477-488.

332. Streten, N.A., 1963: Some observations of Antarctic katabatic winds. Aust. Meteor. Mag., 42, 1-23.

333. Streten, N.A., N. Ishikawa and G. Wendler, 1974: Some observations of the local wind regime on an Alaskan Arctic glacier. Arch. Meteor. Geophys. Bioklim., B22, 337-350.

334. Stull, R.B., 1983: Integral scales for the nocturnal boundary layer. Part II: Heat budget, transport and energy implications. J. Climate Appl. Meteor, 22, 1932-1941. 
335. Stull, R.B., 1991: An Introduction to Boundary Layer Meteorology. Kluwer Academic Publishers, 1991, 666 pp.

336. Sturman, A.P., 1987: Thermal influences on airflow in mountainous terrain. Prog. Phys. Geog., 11, 183-206.

337. Sturman, A.P. and H.A. McGowan, 1995: An assessment of boundary-layer air mass characteristics associated with topographically-induced local wind systems. Bound-Layer Meteor, 74, 181-193.

338. Talman, C.F., 1911: Some mountain winds and their names. Sci. Amer, Suppl. 1831, $71,80-86$.

339. Tang, W., 1976: Theoretical study of cross-valley wind circulation. Arch. Meteor. Geophys. Bioklim., A25, 1-18.

340. Tapp, M. C. and P. W. White, 1976: A non-hydrostatic mesoscale model. Quart. J. Roy. Meteor. Soc., 102, 277-296.

341. Taylor, G.I., 1914: Eddy motion in the atmosphere. Phil. Trans. Roy. Soc. London, Ser. A, 215, 1-26.

342. Taylor, G.I., 1921: Diffusion by continuous movement. Proc. London Math. Soc., Ser. 2, 20, 196-212.

343. Taylor, G.I, 1938: The spectrum of turbulence. Proc. Roy. Soc. London, Ser. A, 164, 476-490.

344. Thorpe, S.A., 1969: Experiments on the stability of stratified shear flows. Radio Sci., 4, 1327-1331.

345. Thorpe, S.A., 1973a: CAT in the lab. Weather, 28, 471-475.

346. Thorpe, S.A., 1973b: Turbulence in stably stratified fluids: A review of laboratory experiments. Bound.-Layer Meteor, 5, 95-119.

347. Thyer, N.H., 1966: A theoretical explanation of mountain and valley winds by a numerical method. Arch. Meteor. Geophys. Bioklim., A15, 318-348.

348. Tollner, H., 1931: Gletscherwinde in den ostalpen. Meteor. Z., 48, 414-421.

349. Tower, W.S., 1903: Mountain and valley breezes. Mon. Wea. Rev., 31, 528-529.

350. Tremback, C.J. and R. Kessler, 1985: A surface temperature and moisture parameterization for use in mesoscale numerical models. Preprints, Seventh Conf. on Numerical Weather Prediction, Montreal, Amer. Meteor. Soc., 355-358.

351. Tremback, C.J. and R.L. Walko, 1992: RAMS Version 2c User's Guide (Draft). Colorado State University, Fort Collins, Colorado 80523.

352. Tripoli, G.J. and W. R. Cotton, 1981: The use of ice-liquid water potential temperature as a thermodynamic variable in deep atmospheric models. Mon. Wea. Rev., 109, 1094-1102.

353. Tripoli, G.J. and W.R. Cotton, 1982: The Colorado State University three-dimensional cloud/mesoscale model-1982. Part I: General theoretical framework and 
sensitivity experiments. J. Rech. Atmos., 16, 185-220.

354. Tripoli, G.J. and W.R. Cotton, 1989: Numerical solution of the Navier-Stokes equations with topography. J. Comput. Phys., 17, 276-310.

355. Turner, J.S., 1973: Buoyancy effects in fluids. Camb. Univ. Press, London, 367 pp.

356. Tyson, P.D., 1968: Velocity fluctuations in the mountain wind. J. Atmos. Sci., 25, 381-384.

357. Tyson, P.D. and R.A. Preston-Whyte, 1972: Observations of regional topographically-induced wind systems in Natal. J. Appl. Meteor, 11, 643-650.

358. Urfer-Henneberger, C. 1967: Zeitliche gesetzmassigkeiten des berg und talwindes. Veroff. Schweiz. Meteor. Zentralanstalt, 4, 245-252.

359. van der Hoven, I., 1957: Power spectrum of horizontal wind speed in the frequency range 0.0007 to 900 cycles per hour. J. Meteor, 14, 160-164.

360. Vergeiner, I., 1987: An elementary valley wind model. Meteor. Atmos. Phys., 36, 255-263.

361. Vergeiner, I. and E. Dreiseitl, 1987: Valley winds and slope winds - Observations and elementary thoughts. Meteor. Atmos. Phys., 36, 264-286.

362. Vergeiner, I., E. Dreiseitl and C.D. Whiteman, 1987: Dynamics of katabatic winds in Colorado's Brush Creek Valley. J. Atmos. Sci., 44, 148-157.

363. Wagner, A., 1931: Klimatologie der freien atmosphare. Handbuch der Klimatologie, W. Koppen und R. Geiger, Hsgbr., Bd. I, Teil F.

364. Wagner, A., 1932a: Hangwind-ausgleichsstroemung-berg und talwind. [Slope wind equalizing flow - mountain and valley wind], Meteor. Z., 49, 209-217.

365. Wagner, A., 1932b: Neue theorie der berg und talwinde. [New theory of mountain and valley winds]. Meteor. Z, 49, 329-341.

366. Wagner, A., 1938: Theorie und beobachtung der periodischen gebirgswinde [Theory and observation of periodic mountain winds]. Beitr. Geophys., 52, 408-449.

367. Walko, R.L., C.J. Tremback, R.A. Pielke and W.R. Cotton, 1995: An interactive nesting algorithm for stretched grids and variable nesting ratios. J. Appl. Meteor, 34, 994-999.

368. Watanabe, T., 1994: Bulk parameterization for a vegetated surface and its application to a simulation of nocturnal drainage flow. Bound.-Layer Meteor, 70, 13-35.

369. Weber, A.H. and R.J. Kurzeja, 1991: Nocturnal planetary boundary layer structure and turbulence episodes during the Project STABLE field program. J. Appl. Meteor, 30, 1117-1133.

370. Weinstock, J., 1978: Vertical turbulent diffusion in a stably stratified fluid. J. Atmos. Sci., 35, 1022-7.

371. Wenger, R., 1923: Zur theorie der berg-und talwinde. [Theory of the mountain and valley wind], Meteor. Z., 40, 193-204. 
372. Whiteman, C.D., 1990: Observations of thermally developed wind systems in mountainous terrain. Meteorological Monograph, Atmospheric Processes over Complex Terrain, 23, No. 45, Blumen, W. Ed., American Meteorological Society, 5-42.

373. Whiteman, C.D., K.J. Allwine, L.J. Fritschen, M.M. Orgill and J.R. Simpson, 1989: Deep valley radiation and surface energy budget microclimates: Part II: Energy budget. J. Appl. Meteor., 28, 427-437.

374. Whiteman, C.D., K.J. Allwine, L.J. Fritschen, M.M. Orgill and J.R. Simpson, 1989: Deep valley radiation and surface energy budget microclimates: Part I: Radiation. J. Appl. Meteor., 28, 414-426.

375. Whiteman, C.D., K.J. Allwine, and J.M. Hubbe, 1991: Winter meteorology of the Grand Canyon Region. Prepared for Salt River Project, Phoenix, Arizona under contract number 16660, March, Battelle Pacific Northwest Laboratories, Richland, WA 99352.

376. Whiteman, C.D. and E. Dreiseitl, 1984: Alpine meteorology: Translations of classic contributions by A. Wagner, E. Ekhart and F. Defant. Atmospheric Studies in Complex Terrain, ASCOT-84-3, Pacific Northwest Laboratory, Richland, Washington, PNL-5141, $121 \mathrm{pp}$.

377. Whiteman, C.D. and J.C. Doran, 1993: The relationship between overlying synopticscale flows and winds within a valley. J. Appl. Meteor, 32, 1669-1682.

378. Whiteman, C.D. and T.B. McKee, 1982: Breakup of temperature inversions in deep mountain valleys: Part II. Thermodynamic model. J. Appl. Meteor., 21, 290-302.

379. Winninghoff, F.J., 1968: On the adjustment toward a geostrophic balance in a simple primitive equation model with application to the problems of intitialization and objective analysis, Ph.D. dissertation, University of California, Los Angeles.

380. Wittich, K.P., 1991: The nocturnal boundary layer over Northern Germany: An observational study. Bound.-Layer Meteor, 55, 47-66.

381. Wolyn, P.G. and McKee, T.B., 1994: The mountain-plains circulation east of a $2 \mathrm{~km}$ high north-south barrier. Mon. Wea. Rev., 122, 1430-1448.

382. Wolyn, P.G. and McKee, T.B., 1992: Modeling and observational study of the daytime evolution east of the crest of the Colorado Rockies. Atmospheric Science Paper No. 496, Department of Atmospheric Science, Colorado State University, Fort Collins, CO 80523.

383. Woods, J.D., 1969: On Richardson's number as a criterion for laminar-turbulent-laminar transition in the ocean and atmosphere. Radio Sci., 4, 1289-1298.

384. Xing-sheng, L., J.E. Gaynor and J.C. Kaimal, 1983: A study of multiple stable layers in the nocturnal lower atmosphere. Bound.-Layer Meteor, 26, 157-168.

385. Yamada, T., 1981: A numerical simulation of nocturnal drainage flow. J. Meteor. Soc. Japan, 59, 108-122.

386. Yamada, T., 1983: Simulations of nocturnal drainage flows by a $\mathrm{q}^{2} 1$ turbulence closure model. J. Atmos. Sci., 40, 91-106. 
387. Yamada, T. and S. Bunker, 1989: A numerical model study of nocturnal drainage flows with strong winds and temperature gradients. J. Appl. Meteor., 28, 545-554.

388. Ye, Z.J., M.Segal, J.R. Garratt and R.A. Pielke, 1989: On the impact of cloudiness on the characteristics of nocturnal downslope flows. Bound.-Layer Meteor., 49, 23-51.

389. Ying, Q. and F. Baopu, 1993: A theoretical study on the interaction between airflow over a mountain and the atmospheric boundary layer. Bound.-Layer Meteor, 64, 101-126.

390. Zeman, O. and N.O. Jensen, 1987: Modification of turbulence characteristics in flow over hills. Quart. J. Roy. Meteor. Soc., 113, 55-80.

391. Zeman, O., 1979: Parameterization of the dynamics of stable boundary layers and nocturnal jets. J. Atmos. Sci., 36, 792-804.

392. Zhong, S. and J.C. Doran, 1995: A modeling study of the effects of inhomogeneous surface fluxes on boundary-layer properties. J. Atmos. Sci., 52, 3129-3142.

393. Zhong, S., J.D. Fast and X. Bian, 1995: A case study of the Great Plains low-level jet using wind profiler network data and a high resolution mesoscale model. Mon. Wea. Rev., (in publication). 


\section{APPENDIX A}

\section{ASCOT 1993 KaTABatic Flow Nights SummaRY}

This appendix contains descriptions, in short hand format, of the local and synoptic conditions of each night of likely drainage winds for the August, 1993 through October 15, 1993 ASCOT observational period. Generally these consist of nearly clear sky, high pressure synoptic situations. The determination of which days would be summarized was made by evaluating synoptic conditions for the entire period and selecting those nights that showed the best drainage characteristics. 3 of the 23 cases reviewed here are used for case studies in this work as described in Section 3.3. Unless the mountainous region is specifically mentioned the descriptions are referring to the conditions on the plains just east of the foothills.

\section{A.1 Case 1: 5-6 Aug 93 (Thur-Fri). Julian Days 217-218}

Previous conditions surface: Weak summertime trough in the region previous 3 days. Overcast to partly cloudy during the day of $5 \mathrm{Aug}$ but no rain reported on the plains or in the mountains to the west of study area.

\section{Surface conditions from 5pm-5am LST (00-12Z 6 Feb):}

$00 \mathrm{Z}$ - Partly to mostly cloudy with dewpoints around $10-12^{\circ} \mathrm{C}$ and temperatures from

$13-24^{\circ} \mathrm{C}$. Winds are $2 \mathrm{~m} \mathrm{~s}^{-1}$ from the northeast. Some weak thunderstorms developed despite high pressure to north moving south.

03Z- High pressure weak over the region, weak stationary front to west. Skies partly cloudy, winds still northeast at $2 \mathrm{~m} \mathrm{~s}^{-1}$. 
$06 \mathrm{Z}-10-13^{\circ} \mathrm{C}$ in the region with skies evolving from partly cloudy to clear. Winds light and variable.

$09 \mathrm{Z}$ - Weak trough north, still high pressure in area, temperatures in the mid-50's.

Skies are partly cloudy to clear and winds are light and variable.

$12 \mathrm{Z}$ - Partly cloudy skies and temperatures in the around $11^{\circ} \mathrm{C}$. Sea level adjusted pressure is $1017 \mathrm{mb}$, clear and calm to the west. Winds at Denver $2 \mathrm{~m} \mathrm{~s}^{-1}$ from westnorthwest.

\section{A.2 Case 2: 22-23 Aug 93 (Sun-Mon). Julian Days 234-235}

Previous conditions surface: A weak frontal passage during the day Sunday causes temperatures to drop from the around $25^{\circ} \mathrm{C}$ to around $24^{\circ} \mathrm{C}$ and the dewpoint from the around $9^{\circ} \mathrm{C}$ to around $2^{\circ} \mathrm{C}$. Winds from west-northwest at $4.5 \mathrm{~m} \mathrm{~s}^{-1}$ in study region. Skies are partly to mostly cloudy.

Surface conditions from 5pm-5am LST (00-12Z 23 Aug):

$00 \mathrm{Z}$ - Winds $4.5 \mathrm{~m} \mathrm{~s}^{-1}$ from west, around $24^{\circ} \mathrm{C}$ and partly cloudy, pressure $1008 \mathrm{mb}$ and rising.

$\mathbf{0 3 Z}$ - Clear to partly cloudy with temperatures in around $18^{\circ} \mathrm{C}$, light variable winds.

Weak trough lingers to the south and east of the study area.

$06 \mathrm{Z}$ - Trough gone. Clear skies and calm (Denver) to $2 \mathrm{~m} \mathrm{~s}^{-1}$ winds of variable direction. Temperatures around $17^{\circ} \mathrm{C}$.

$09 Z$ - No information available.

$12 \mathrm{Z}$ - Clear to partly cloudy with $2 \mathrm{~m} \mathrm{~s}^{-1}$ westerly winds. Lows down to around $12^{\circ} \mathrm{C}$. $1014 \mathrm{mb}$.

Post case conditions: Calm weak gradient conditions continue. Mostly clear.

\section{A.3 Case 3: 23-24 Aug 93 (Mon-Tue). Julian Days 235-236}

Previous conditions surface: Warm clear day with temperatures around $27^{\circ} \mathrm{C}$. Light winds near $2 \mathrm{~m} \mathrm{~s}^{-1}$ and pressure at Denver at $21 \mathrm{Z} 1008 \mathrm{mb}$.

Surface conditions from 5pm-5am LST (00-12Z 24 Aug):

$\mathbf{0 0 Z}$ - Light variable winds, around $27^{\circ} \mathrm{C}$ dewpoint around $-1^{\circ} \mathrm{C}$. Clear skies.

$\mathbf{0 3 Z}$ - Cooling fast temperatures around $21^{\circ} \mathrm{C}$ with clear skies and light south-south- 
east winds at $2 \mathrm{~m} \mathrm{~s}^{-1} .1010 \mathrm{mb}$ in Denver.

$06 Z$ - Clear, high pressure (weak) to southwest over Telluride. west-southwest winds in study area temperatures around $18^{\circ} \mathrm{C}$.

$09 \mathrm{Z}$ - Clear, around $10-15^{\circ} \mathrm{C}$, winds south-westerly and $2.0-4.5 \mathrm{~m} \mathrm{~s}^{-1}$.

$12 \mathrm{Z}$ - Clear and $12-15^{\circ} \mathrm{C}$. Winds light and west to southwest.

Post case conditions: Clear, temperatures reach around $32^{\circ} \mathrm{C}$ with around $-1^{\circ} \mathrm{C}$ dewpoints.

\section{A.4 Case 4: 24-25 Aug 93 (Tue-Wed). Julian Days 236-237}

Previous conditions surface: Described above.

\section{Surface conditions from 5pm-5am LST (00-12Z 25 Aug):}

$00 \mathrm{Z}$ - High around $27^{\circ} \mathrm{C}$, light southeast winds dewpoints around $6^{\circ} \mathrm{C}$, clear.

$\mathbf{0 3 Z}$ - Clear to partly cloudy, temperatures around $24^{\circ} \mathrm{C}$, light southeast winds, small surface pressure gradient.

$06 \mathrm{Z}$ - Mostly clear, $21-25^{\circ} \mathrm{C}$, light south-southeast winds.

$09 Z$ - Approaching front from northwest in central Wyoming. south-southeast winds at $2.0-4.5 \mathrm{~m} \mathrm{~s}^{-1}$ with pressure dropping to 1005 from 1008 over near 6 hours. Still clear.

$12 \mathrm{Z}$ - Front still north, near Cheyenne, Wyoming, winds south at $2 \mathrm{~m} \mathrm{~s}^{-1}$. Mostly clear. Post case conditions: Weak frontal passage, temperatures reach around $30^{\circ} \mathrm{C}$ again. Lee trough develops and winds up to $9 \mathrm{~m} \mathrm{~s}^{-1}$ around trough.

\section{A.5 Case 5: 31 Aug - 01 Sep 93 (Mon-Tue). Julian DaYs 243-244}

Previous conditions surface: Frontal passage early Monday with a high pressure of $1020 \mathrm{mb}$ strength following. Temperatures around $15^{\circ} \mathrm{C}$ with dewpoints in the around $-1^{\circ} \mathrm{C}$.

\section{Surface conditions from 5pm-5am LST (00-12Z 01 Aug):}

$\mathbf{0 0 Z}$ - High pressure over study area with light variable winds. Partly to mostly cloudy. 03Z - High right over study area with clear to partly cloudy skies. Temperatures around $9^{\circ} \mathrm{C}$, winds light and variable at $2 \mathrm{~m} \mathrm{~s}^{-1}$, westerly at Denver at $2 \mathrm{~m} \mathrm{~s}^{-1}$. Dewpoints around $-1^{\circ} \mathrm{C}$. 
$\mathbf{0 6 Z}$ - Clear to partly cloudy with around $9^{\circ} \mathrm{C}$, high pressure slightly east of study area. Light variable winds.

$09 Z$ - Partly cloudy to clear and $6-10^{\circ} \mathrm{C}$. Mostly east component winds. Cold for August.

$12 \mathrm{Z}-0^{\circ} \mathrm{C}$ in Craig, $3^{\circ} \mathrm{C}$ in Cheyenne, $2^{\circ} \mathrm{C}$ in Akron. $1025 \mathrm{mb}$ high over Limon. Dewpoints in around $-1^{\circ} \mathrm{C}$.

Post case conditions: Partly cloudy with temperatures up to $23^{\circ} \mathrm{C}$. southeast winds at $2.0-4.5 \mathrm{~m} \mathrm{~s}^{-1}$ indicative of diurnal cycle. Dewpoints high around $-1-7^{\circ} \mathrm{C}$. High moving toward lowa.

\section{A.6 Case 6: 02-03 Sep 93 (Thu-Fri). Julian Days 245-246}

Previous conditions surface: Weak frontal passage Wednesday night with high pressure building in behind. Partly to mostly cloudy skies with some showers overnight Wednesday. Temperatures up to around $15^{\circ} \mathrm{C}$.

Surface conditions from 5pm-5am LST (00-12Z 03 Sep):

$00 \mathrm{Z}-1020 \mathrm{mb}$ high right over region with temperatures in around $15^{\circ} \mathrm{C}$, with near $20^{\circ} \mathrm{C}$ in the mountains. Dewpoints are around $7^{\circ} \mathrm{C}$ and skies are partly cloudy.

$\mathbf{0 3 Z}$ - Clear skies, around $11^{\circ} \mathrm{C}$ all over with light variable winds near $2 \mathrm{~m} \mathrm{~s}^{-1} .1023$ mb sea level pressure.

$06 \mathrm{Z}$ - High right over area, temperatures $9-11^{\circ} \mathrm{C}$, light northeast winds most places. $09 \mathrm{Z}$ - Clear skies, around $7^{\circ} \mathrm{C}$ with light variable winds. $1024 \mathrm{mb}$ high. around $-1^{\circ} \mathrm{C}$ in mountains.

$12 \mathrm{Z}$ - Clear skies, temperatures around $8^{\circ} \mathrm{C}$ on the plains, temperatures around $-1^{\circ} \mathrm{C}$ mountains. high has moved south to Pueblo at $1027 \mathrm{mb}$. Light south-southwest winds $<2 \mathrm{~m} \mathrm{~s}^{-1}$.

Post case conditions: Clear with temperatures up to around $27^{\circ} \mathrm{C}$ with dewpoints $7^{\circ} \mathrm{C}$ to $-1^{\circ} \mathrm{C}$. Mountains reach around $25^{\circ} \mathrm{C}$. Plains winds southeast indicating upslope. Pressure falls to near $1015 \mathrm{mb}$.

\section{A.7 CaSe 7: 03-04 Sep 93 (Fri-Sat). Julian Days 246-247}

Previous conditions surface: Described above. 


\section{Surface conditions from 5pm-5am LST (00-12Z 04 Sep):}

$\mathbf{0 0 Z}$ - Low around $27^{\circ} \mathrm{C}$ dewpoints around $-3^{\circ} \mathrm{C}$ to $0^{\circ} \mathrm{C}$ (very dry). Winds southeast indicating upslope at $2 \mathrm{~m} \mathrm{~s}^{-1}$. Slight trough running north-south through Limon. $\mathbf{0 3 Z}$ - Calm to light winds on plains at $2 \mathrm{~m} \mathrm{~s}^{-1}$ and variable. Around the region temperatures are near $20^{\circ} \mathrm{C}$ with a typical sea level pressure of $1017 \mathrm{mb}$. Skies are clear. $06 \mathrm{Z}$ - Still clear, slight trough to the west. Temperatures are near $15^{\circ} \mathrm{C}$. Winds light and variable near $2 \mathrm{~m} \mathrm{~s}^{-1}$.

$09 \mathrm{Z}$ - Clear, light variable winds with temperatures around $13^{\circ} \mathrm{C}$, near $5^{\circ} \mathrm{C}$ in mountains.

$12 \mathrm{Z}$ - Still clear with light variable winds and around $11^{\circ} \mathrm{C}$.

Post case conditions: Clear to partly cloudy with temperatures reaching around $27^{\circ} \mathrm{C}$ with dewpoints in the around $-1^{\circ} \mathrm{C}$. Southeast winds and a cold front well to the north.

\section{A.8 Case 8: 07-08 Sep 93 (Tue-Wed). Julian Days 250-251}

Previous conditions surface: Weak frontal passage overnight Monday with showers dissipating toward case night (Tuesday night). Cloudy skies most of the day, but high pressure approaching.

Surface conditions from 5pm-5am LST (00-12Z 08 Sep):

$\mathbf{0 0 Z}$ - Overcast to breaks in overcast with showers in the Denver area. Indications of a weak trough exiting the area. Clearing skies to west. around $12^{\circ} \mathrm{C}$ with dewpoints in the around $11^{\circ} \mathrm{C}$. Winds are variable up to $4.5 \mathrm{~m} \mathrm{~s}^{-1}$.

03Z - Partly to mostly cloudy plains, clear west. Temperatures around $11^{\circ} \mathrm{C}$ and northeasterly winds at $2.0-4.5 \mathrm{~m} \mathrm{~s}^{-1}$. A $1024 \mathrm{mb}$ high resides over Limon.

$06 \mathrm{Z}$ - Clear to partly cloudy with temperatures near $10^{\circ} \mathrm{C}$. Light variable winds.

$09 \mathrm{Z}$ - Dewpoints dropping slowly as with lows fall to just under $10^{\circ} \mathrm{C}$. Laramie is $2^{\circ} \mathrm{C}$ with $-1^{\circ} \mathrm{C}$ dewpoint. Mountains in around $6^{\circ} \mathrm{C}$. Light variable winds. Fog possible with $1021 \mathrm{mb}$ common.

$12 \mathrm{Z}$ - Clear to slightly cloudy with most temperatures in around $6^{\circ} \mathrm{C}$. Light variable winds. Mountains $2^{\circ} \mathrm{C}$ with $-1^{\circ} \mathrm{C}$ dewpoint.

Post case conditions: Clear, temperatures reach $22^{\circ} \mathrm{C}$ with around $0^{\circ} \mathrm{C}$ dewpoints. Some surface pressure gradient approaching with a strong cold front in northern Mon- 
tana, northwest winds near $4.5 \mathrm{~m} \mathrm{~s}^{-1}$.

\section{A.9 CASE 9: 08-09 Sep 93 (Wed-Thu). Julian Days 251-252}

Previous conditions surface: Described above.

\section{Surface conditions from 5pm-5am LST (00-12Z 09 Sep):}

$\mathbf{0 0 Z}$ - Clear to slightly cloudy with around $24^{\circ} \mathrm{C}$ and near $-1^{\circ} \mathrm{C}$ dewpoints. Variable winds up to $4.5 \mathrm{~m} \mathrm{~s}^{-1}$.

$03 \mathrm{Z}$ - Clear, around $15^{\circ} \mathrm{C}$ and light variable winds of about $2 \mathrm{~m} \mathrm{~s}^{-1}$. Frontal gradient close and actual front in soutern Montana.

$06 Z$ - Front in central Wyoming, but clear skies in study area. Light variable winds with around $12^{\circ} \mathrm{C}$ and calm in the mountains (around $6^{\circ} \mathrm{C}$ ). Pressures near $1012 \mathrm{mb}$. $12 Z$ - Front approaching Laramie but weakened allowing variable winds up to $7 \mathrm{~m} \mathrm{~s}^{-1}$. Clear and around $12^{\circ} \mathrm{C}$.

Post case conditions: Frontal passage at $\sim 15 Z$ but mostly clear conditions anyway. No showers but winds become northwest at up to $7 \mathrm{~m} \mathrm{~s}^{-1}$. Temperatures around $25^{\circ} \mathrm{C}$.

\section{A.10 CASE 10: 10-11 SEP 93 (Fri-SAT). JUlian DaYs 253-254}

Previous conditions surface: Lingering stationary front along Front Range with variable clouds that eventually dissipate. Temperatures up to around $21^{\circ} \mathrm{C}$ with dewpoints around $7^{\circ} \mathrm{C}$.

Surface conditions from 5pm-5am LST (00-12Z 11 Sep):

$00 Z$ - Clear to partly cloudy with temperatures $23-26^{\circ} \mathrm{C}$ and dewpoints in the around $6^{\circ} \mathrm{C}$ plains, around $-3^{\circ} \mathrm{C}$ mountains. Shower in Denver, winds light and variable up to $2 \mathrm{~m} \mathrm{~s}^{-1}$.

$\mathbf{0 3 Z}$ - Trough eastern Colorado but clear to partly cloudy. Around $18^{\circ} \mathrm{C}$ with winds variable up to $4.5 \mathrm{~m} \mathrm{~s}^{-1}$.

$06 \mathrm{Z}$ - Around $17^{\circ} \mathrm{C}$ with near $8^{\circ} \mathrm{C}$ mountains. Clear, light generally south winds. $09 Z$ - Mostly southwest winds on plains up to $7 \mathrm{~m} \mathrm{~s}^{-1}$ and clear. Temperatures near $14^{\circ} \mathrm{C}$ on plains, near $5^{\circ} \mathrm{C}$ mountains (some calm areas). Pressures near $1010 \mathrm{mb}$. $12 \mathrm{Z}$ - Trough still in eastern Colorado, winds southerly and westerly at $2.0-4.5 \mathrm{~m} \mathrm{~s}^{-1}$. Around $12^{\circ} \mathrm{C}$ and mostly clear (around $-1{ }^{\circ} \mathrm{C}$ mountains). 
Post case conditions: Partly cloudy temperatures reach high around $27^{\circ} \mathrm{C}$ with westerly component winds up to $9 \mathrm{~m} \mathrm{~s}^{-1}$ and dewpoints around $-2^{\circ} \mathrm{C}$.

\section{A.11 Case 11: 11-12 Sep 93 (SAT-Sun). Julian Days 254-255}

This case night was just before the first snowstorm of the 1993-1994 cold season.

Previous conditions surface: Described above.

Surface conditions from 5pm-5am LST (00-12Z 12 Sep):

$00 Z$ - Geostrophic wind component from northwest and surface winds were mostly northwesterly (with some south component) at $2 \mathrm{~m} \mathrm{~s}^{-1}$. Dewpoints around $-2^{\circ} \mathrm{C}$ and temperatures up to $30^{\circ} \mathrm{C}$. Mountains had a westerly component at $7 \mathrm{~m} \mathrm{~s}^{-1}$ with near $25^{\circ} \mathrm{C} .1003 \mathrm{mb}$ pressure.

$\mathbf{0 3 Z}$ - Clear to slightly cloudy with variable winds near $2 \mathrm{~m} \mathrm{~s}^{-1}$. Temperatures down to around $21^{\circ} \mathrm{C}$.

$06 \mathrm{Z}$ - Clear to slightly cloudy with south and southwest winds up to $4.5 \mathrm{~m} \mathrm{~s}^{-1}$. Temperatures near around $18^{\circ} \mathrm{C}$. Front crossing Yellowstone running northeast-southwest. $09 \mathrm{Z}$-Temperatures around $18^{\circ} \mathrm{C}$ plains, around $8^{\circ} \mathrm{C}$ mountains, clear. Winds southsouthwest on plains, cross-isobaric toward Low in central South Dakota (992 mb). Calm mountains.

$12 \mathrm{Z}$ - Front across Central Wyoming, clear and near $14^{\circ} \mathrm{C}$ on plains, around $6^{\circ} \mathrm{C}$ mountains. Variable southerly component winds on plains up to $4.5 \mathrm{~m} \mathrm{~s}^{-1}$. Pressure dropping to $998 \mathrm{mb}$, front approaching fast.

Post case conditions: Mostly clear with temperatures around $32^{\circ} \mathrm{C}$ by afternoon. Only around $8^{\circ} \mathrm{C}$ in northern Wyoming behind two fronts. $4.5 \mathrm{~m} \mathrm{~s}^{-1}$ winds primarily south on plains.

\section{A.12 Case 12: 14-15 Sep 93 (Tue-Wed). Julian Days 257-258}

Previous conditions surface: Snowstorm on foothills and plains. 4" near Denver and east and > 6" above $2000 \mathrm{~m}$ MSL 20" on Longs Peak. Snow cover could augment drainage via additional radiational cooling. Highs up to around $15^{\circ} \mathrm{C}$ with northwest winds up to $4.5 \mathrm{~m} \mathrm{~s}^{-1}$. Clear to partly cloudy during day before (Tuesday afternoon). Surface conditions from 5pm-5am LST (00-12Z 15 Sep): 
00Z - Clear to slightly cloudy, around $18^{\circ} \mathrm{C}$, dewpoints around $0^{\circ} \mathrm{C}$ plains, around $5^{\circ} \mathrm{C}$ mountains. A high pressure system resides in central Kansas at $1023 \mathrm{mb}$. Wind light and variable.

$\mathbf{0 3 Z}$ - Around $12^{\circ} \mathrm{C}$, clear, wind variable up to $4.5 \mathrm{~m} \mathrm{~s}^{-1}$.

$06 \mathrm{Z}$ - Near $10^{\circ} \mathrm{C}$, around $2^{\circ} \mathrm{C}$ mountains. Weak broken warm front analyzed across northeast Wyoming and Colorado. Clear. Winds variable up to $4.5 \mathrm{~m} \mathrm{~s}^{-1}$. $09 \mathrm{Z}$ - Around $7^{\circ} \mathrm{C}$, around $-2^{\circ} \mathrm{C}$ mountains, clear. Pressure gradient has developed northwest-southeast near $4 \mathrm{mb}$ across Colorado.

$12 \mathrm{Z}$ - Low-around $7^{\circ} \mathrm{C}\left(-3^{\circ} \mathrm{C}\right.$ in Gunnison), clear. Weakened pressure gradient.

Post case conditions: Clear, temperatures reach $23^{\circ} \mathrm{C}$ with around $-4^{\circ} \mathrm{C}$ dewpoints. Low tracks across northern Wyoming giving gradient across Colorado. Weak trough crosses Colorado west-east.

\section{A.13 Case 13: 15-16 SeP 93 (Wed-Thu). Julian Days 258-259}

Previous conditions surface: Described above.

Surface conditions from 5pm-5am LST (00-12Z 00 Sep):

00Z - Temperatures around $25^{\circ} \mathrm{C}$ and clear to slightly cloudy. Dewpoints around $-2^{\circ} \mathrm{C}$ and winds variable up to $4.5 \mathrm{~m} \mathrm{~s}^{-1}$. A weak trough is set up north-south along the Front Range and a weak low is tracking into east South Dakota with a trailing cold front. $03 Z$ - Clear, around $18^{\circ} \mathrm{C}$, weak cold front approaches from central Wyoming. $06 \mathrm{Z}$ - Pressure still near $1010 \mathrm{mb}$. Temperatures around $12^{\circ} \mathrm{C}$, Westerly wind in Denver, trough analyzed north-south in east Colorado. Wind up to $2 \mathrm{~m} \mathrm{~s}^{-1}$.

$09 \mathrm{Z}$ - Front not analyzed. Clear, around $11^{\circ} \mathrm{C}$ and around $-1{ }^{\circ} \mathrm{C}$ in mountains. Slightly cloudy.

$12 Z$ - Front analyzed through Denver with a wind shift, though still clear to slightly cloudy. Winds now have northerly component up to $7 \mathrm{~m} \mathrm{~s}^{-1}$.

Post case conditions: Mostly cloudy, temperatures up to $20^{\circ} \mathrm{C}$. Front continues southward to Pueblo.

\section{A.14 Case 14: 18-19 Sep 93 (Sun-Mon). Julian Days 261-262}

Previous conditions surface: Low recently pulled through now in northeast. Mostly 
cloudy skies and generally northwest winds at up to $9 \mathrm{~m} \mathrm{~s}^{-1}$. Calming rapidly as gradient moves east.

Surface conditions from 5pm-5am LST (00-12Z $00 \mathrm{Sep})$ :

$\mathbf{0 0 Z}$ - Temperatures are around $18^{\circ} \mathrm{C}$, northwest winds at up to $7 \mathrm{~m} \mathrm{~s}^{-1}$. High $(1015$ $\mathrm{mb}$ ) over Grand Junction. Low around $-1{ }^{\circ} \mathrm{C}$ dewpoints around $-6^{\circ} \mathrm{C}$.

$03 Z$ - Clear to slightly cloudy. Calm to light variable winds and around $12^{\circ} \mathrm{C}$.

$06 \mathrm{Z}$ - Calm to westerly-southwest winds at up to $2 \mathrm{~m} \mathrm{~s}^{-1}$. Near $10^{\circ} \mathrm{C}$.

$09 \mathrm{Z}$ - Around $7^{\circ} \mathrm{C}$, clear, variable winds up to $4.5 \mathrm{~m} \mathrm{~s}^{-1}$. High over Gunnison. Near $1^{\circ} \mathrm{C}$ in mountains.

$12 \mathrm{Z}$ - Around $7^{\circ} \mathrm{C}$ on plains, clear to slightly cloudy, around $-2^{\circ} \mathrm{C}$ mountains. $1016 \mathrm{mb}$ representative.

Post case conditions: Clear, high pressure, temperatures up to $22^{\circ} \mathrm{C}$ with dewpoints around $-2^{\circ} \mathrm{C}$.

\section{A.15 Case 15: 20-21 Sep 93 (Mon-Tue). Julian Days 263-264}

Previous conditions surface: Described above.

\section{Surface conditions from 5pm-5am LST (00-12Z 21 Sep):}

$\mathbf{0 0 Z}$ - around $24^{\circ} \mathrm{C}$, easterly component winds (with slight southerly tendency) up to $4.5 \mathrm{~m} \mathrm{~s}^{-1}$. Clear.

$\mathbf{0 3 Z}$ - Clear to slightly cloudy, around $15^{\circ} \mathrm{C}$ and near $10^{\circ} \mathrm{C}$ mountains. Dewpoints around $2^{\circ} \mathrm{C}$, wind south-southeast up to $4.5 \mathrm{~m} \mathrm{~s}^{-1}$.

$06 Z$ - Clear, around $11^{\circ} \mathrm{C}$, wind southeast at up to $4.5 \mathrm{~m} \mathrm{~s}^{-1}$. East wind mountains with temperatures $6-10^{\circ} \mathrm{C}$.

$09 \mathrm{Z}$ - Clear, near $10^{\circ} \mathrm{C}$, variable winds up to $2 \mathrm{~m} \mathrm{~s}^{-1}$. Calm at Eagle and $5^{\circ} \mathrm{C}$.

$12 Z$ - Weak front approaching northwest corner of Colorado and $1011 \mathrm{mb}$ typical. Around $-1{ }^{\circ} \mathrm{C}$ mountains, around $12^{\circ} \mathrm{C}$ plains and clear to slightly cloudy.

Post case conditions: A weak trough lingers as the front approaches. Partly cloudy skies develop after clear morning with temperatures up to around $25^{\circ} \mathrm{C}$. Dewpoints still low near $-4^{\circ} \mathrm{C}$. 


\section{A.16 Case 16: 26-27 Sep 93 (Sun-Mon). Julian Days 269-270}

Previous conditions surface: Increasing high pressure after frontal passage Saturday. Partly cloudy skies give way to clearing with highs in the around $15^{\circ} \mathrm{C}$. Gradient reduces.

Surface conditions from 5pm-5am LST (00-12Z 27 Sep):

$00 \mathrm{Z}$ - Weak gradient and clear skies. $1023 \mathrm{mb}$ typical. around $18^{\circ} \mathrm{C}$.

$\mathbf{0 3 Z}$ - Clear, near $10^{\circ} \mathrm{C}$, light southwest winds up to $2 \mathrm{~m} \mathrm{~s}^{-1}$.

$06 \mathrm{Z}$ - Clear, around $7^{\circ} \mathrm{C}$, light variable wind.

$09 \mathrm{Z}$ - Clear, around $9^{\circ} \mathrm{C}$, winds light and variable. Near $-1^{\circ} \mathrm{C}$ mountains.

$12 \mathrm{Z}$ - Clear around $7^{\circ} \mathrm{C}$ with light variable winds.

Post case conditions: Clear, southeast winds up to $4.5 \mathrm{~m} \mathrm{~s}^{-1}$, around $25^{\circ} \mathrm{C}$, dewpoint temperatures are near $-5^{\circ} \mathrm{C}$.

\section{A.17 CaSe 17: 27-28 Sep 93 (Mon-Tue). Julian Days 270-271}

Previous conditions surface: Described above.

Surface conditions from 5pm-5am LST (00-12Z 28 Sep):

$00 \mathrm{Z}$ - Clear to slightly cloudy, temperatures near $25^{\circ} \mathrm{C}$, winds with east component. Westerly component in mountains.

03Z - Around $17^{\circ} \mathrm{C}$, light variable winds up to $2 \mathrm{~m} \mathrm{~s}^{-1}$, clear. Near $10^{\circ} \mathrm{C}$ mountains with near $-7^{\circ} \mathrm{C}$ dewpoints.

$06 \mathrm{Z}$ - Light variable winds (some calm), around $11^{\circ} \mathrm{C}$. Front approaching from northeast as defined by wind shift, not much weather.

$09 \mathrm{Z}$ - Clear, around $10^{\circ} \mathrm{C}$, light variable winds, around $0^{\circ} \mathrm{C}$ mountains.

$12 \mathrm{Z}$ - Clear around $7^{\circ} \mathrm{C}$, light winds. Weak front hangs in northeast Colorado. Temperatures are around $-3^{\circ} \mathrm{C}$ in the mountains. $1019 \mathrm{mb}$ typical.

Post case conditions: Partly to mostly cloudy with weak frontal passage. Temperatures near $18^{\circ} \mathrm{C}$.

\section{A.18 CaSe 18: 29-30 Sep 93 (Wed-Thu). Julian Days 272-273}

Previous conditions surface: Clear to partly cloudy with weak front analyzed along the Front Range. Around $24^{\circ} \mathrm{C}$ with westerly component winds up to $7 \mathrm{~m} \mathrm{~s}^{-1}$. 
Surface conditions from 5pm-5am LST (00-12Z 30 Sep):

$00 \mathrm{Z}$ - Stationary front still along Front Range. Winds southeast at up to $7 \mathrm{~m} \mathrm{~s}^{-1}$. near $24^{\circ} \mathrm{C}, 1016 \mathrm{mb}$ typical.

$03 \mathrm{Z}$ - Stationary front still along Front Range; around $15^{\circ} \mathrm{C}$, southeast winds on plains, northeast winds in mountains.

$06 Z$ - Front now near Kansas border, winds on plains now southwesterly up to $7 \mathrm{~m} \mathrm{~s}^{-1}$. Clear to partly cloudy.

$09 \mathrm{Z}$ - Clear to slightly cloudy, around $12^{\circ} \mathrm{C}$, around $0^{\circ} \mathrm{C}$ mountains, dewpoints plains around $-1^{\circ} \mathrm{C}$. Wind southwesterly up to $4.5 \mathrm{~m} \mathrm{~s}^{-1}$.

$12 \mathrm{Z}$ - Clear, calm at Eagle. Southwest-northeast pressure gradient entering Colorado from north, westerly component wind up to $4.5 \mathrm{~m} \mathrm{~s}^{-1}$. Near $10^{\circ} \mathrm{C}$ plains, around $-2^{\circ} \mathrm{C}$ mountains.

Post case conditions: Slightly to mostly cloudy as pressure gradient enters north Colorado. Weak trough associated clouds skies. Winds up to $11 \mathrm{~m} \mathrm{~s}^{-1}$ from the northwest and near $25^{\circ} \mathrm{C}$.

\section{A.19 CASE 19: 02-03 OCT 93 (SAT-Sun). Julian DAYS 275-276}

Previous conditions surface: Weak front exiting region with around $18^{\circ} \mathrm{C}$ and 1020 mb typical. Slightly cloudy but clearing.

\section{Surface conditions from 5pm-5am LST (00-12Z 03 Oct):}

$\mathbf{0 0 Z}$ - Around $17^{\circ} \mathrm{C}$, slightly cloudy to clear, southwest winds up to $4.5 \mathrm{~m} \mathrm{~s}^{-1} .1023 \mathrm{mb}$ common pressure.

03Z - Clear, around $11^{\circ} \mathrm{C}$ plains, around $9^{\circ} \mathrm{C}$ mountains. Wind dying from southwest nearing variable and light.

$06 \mathrm{Z}$ - Around $9^{\circ} \mathrm{C}$, dewpoints around $-1{ }^{\circ} \mathrm{C}$, clear, light variable winds. Near $5^{\circ} \mathrm{C}$ mountains, southeast winds.

$09 \mathrm{Z}$ - Clear, around $7^{\circ} \mathrm{C}$ on plains with southeast winds up to $2 \mathrm{~m} \mathrm{~s}^{-1}$.

$12 \mathrm{Z}$ - Southwest winds up to $4.5 \mathrm{~m} \mathrm{~s}^{-1}$, around $6^{\circ} \mathrm{C}$ plains, around $0^{\circ} \mathrm{C}$ mountains. $1022 \mathrm{mb}$ pressure normal.

Post case conditions: Clear, around $27^{\circ} \mathrm{C}, 1016 \mathrm{mb}$ or so. Winds variable up to $2 \mathrm{~m} \mathrm{~s}^{-}$ 1 . 


\section{A.20 CaSe 20: 03-04 OCt 93 (Sun-Mon). Julian Days 276-277}

Previous conditions surface: Described above.

Surface conditions from 5pm-5am LST (00-12Z 04 Oct):

$00 \mathrm{Z}$ - Clear to slightly cloudy, around $25^{\circ} \mathrm{C}$, dewpoints around $-3^{\circ} \mathrm{C}$. Northeast wind up to $2 \mathrm{~m} \mathrm{~s}^{-1}$. West wind in mountains up to $4.5 \mathrm{~m} \mathrm{~s}^{-1}$ with dewpoints near $-7^{\circ} \mathrm{C} .1017$ $\mathrm{mb}$.

$\mathbf{0 3 Z}$ - Clear, around $16^{\circ} \mathrm{C}$, south-southwest winds near $2 \mathrm{~m} \mathrm{~s}^{-1}$.

$06 Z$ - Clear, southeast-northeast winds up to $2 \mathrm{~m} \mathrm{~s}^{-1}$. Low-around $12^{\circ} \mathrm{C}$, high around $1{ }^{\circ} \mathrm{C}$ mountains.

$09 \mathrm{Z}$ - Clear, $1016 \mathrm{mb}$ common, light variable winds, calm at Craig. Around $10^{\circ} \mathrm{C}$ plains, around $0^{\circ} \mathrm{C}$ in mountains.

$12 Z$ - Clear, near $10^{\circ} \mathrm{C}$, dewpoints near $-1^{\circ} \mathrm{C}$, winds light and variable.

Post case conditions: Clear, front approaching from northeast, high in around $25^{\circ} \mathrm{C}$, southeast winds light.

\section{A.21 CaSe 21: 04-05 OCT 93 (Mon-Tue). Julian Days 277-278}

Previous conditions surface: Described above.

\section{Surface conditions from 5pm-5am LST (00-12Z 05 Oct):}

$\mathbf{0 0 Z}$ - Front in northeast corner of Colorado, slight trough along Front Range, up to $26^{\circ} \mathrm{C}$. south-east winds up to $4.5 \mathrm{~m} \mathrm{~s}^{-1}$, slightly cloudy to clear. Near $24^{\circ} \mathrm{C}$ mountains, dewpoints at $-6^{\circ} \mathrm{C}$.

03Z - Front lingers northeast Colorado trough still along Front Range. Slightly cloudy to clear, around $18^{\circ} \mathrm{C}$. East component winds up to $4.5 \mathrm{~m} \mathrm{~s}^{-1}$.

$06 \mathrm{Z}$ - Around $12^{\circ} \mathrm{C}$, clear, east component winds up to $2 \mathrm{~m} \mathrm{~s}^{-1}$. Mountains near $5^{\circ} \mathrm{C}$. $09 \mathrm{Z}$ - Around $12^{\circ} \mathrm{C}$, clear, southwest winds up to $2 \mathrm{~m} \mathrm{~s}^{-1}$. $12 \mathrm{Z}$ - Slightly cloudy to clear, around $12^{\circ} \mathrm{C}$, south component winds up to $4.5 \mathrm{~m} \mathrm{~s}^{-1}$. Front still lingers weakly in northeast Colorado, trough still weak along Front Range. Post case conditions: Slightly to partly cloudy, south component winds, near $30^{\circ} \mathrm{C}$. Trough along Kansas border. 


\section{A.22 CaSe 22: 05-06 OCT 93 (Tue-Wed). Julian Days 278-279}

Previous conditions surface: Described above.

Surface conditions from 5pm-5am LST (00-12Z 06 Oct):

$\mathbf{0 0 Z}$ - Slightly to partly cloudy (Denver at $50 \%$ cloud cover), near $25^{\circ} \mathrm{C}$, southeast winds up to $4.5 \mathrm{~m} \mathrm{~s}^{-1}$.

$\mathbf{0 3 Z}$ - Dewpoints around $-3^{\circ} \mathrm{C}$, around $20^{\circ} \mathrm{C}$, south-east winds up to $2 \mathrm{~m} \mathrm{~s}^{-1}$. Mountains near $10^{\circ} \mathrm{C}$ with east winds. High pressure over Cameron Pass at $1015 \mathrm{mb}$.

$06 \mathrm{Z}$ - Clear, around $12^{\circ} \mathrm{C}$, south component winds up to $2 \mathrm{~m} \mathrm{~s}^{-1}$.

$09 \mathrm{Z}$ - Clear, near $10^{\circ} \mathrm{C}$, variable winds up to $4.5 \mathrm{~m} \mathrm{~s}^{-1}$, mountains around $5^{\circ} \mathrm{C}$.

$12 \mathrm{Z}$ - Clear to partly cloudy, around $11^{\circ} \mathrm{C}$, variable winds near $2 \mathrm{~m} \mathrm{~s}^{-1}$. Mountains partly cloudy and calm near $5^{\circ} \mathrm{C}$.

Post case conditions: Partly to mostly cloudy or overcast, up to around $25^{\circ} \mathrm{C}$.

\section{A.23 CaSe 23: 10-11 OCt 93 (Sun-Mon). Julian Days 283-284}

Previous conditions surface: Exiting upslope, partly convective system. Clearing all day Sunday. Around $12^{\circ} \mathrm{C}$ and variable cool winds, shifting to $9 \mathrm{~m} \mathrm{~s}^{-1}$ northwest in the afternoon. Dewpoints around $-3^{\circ} \mathrm{C}$.

Surface conditions from 5pm-5am LST (00-12Z 11 Oct):

$00 \mathrm{Z}$ - Partly to slightly cloudy, around $12^{\circ} \mathrm{C}$, variable winds up to $7 \mathrm{~m} \mathrm{~s}^{-1}$. High pressure $(1022 \mathrm{mb})$ to northwesterly.

$\mathbf{0 3 Z}$ - Variable winds up to $2 \mathrm{~m} \mathrm{~s}^{-1}$ on plains with around $6^{\circ} \mathrm{C}$. Southeast winds up to 2 $\mathrm{m} \mathrm{s}^{-1}$, mountains around $2^{\circ} \mathrm{C}$.

$1019 \mathrm{mb}$ common, high near Grand Junction. Clear.

$06 \mathrm{Z}$ - Temperatures near $5^{\circ} \mathrm{C}$ with light variable winds up to $2 \mathrm{~m} \mathrm{~s}^{-1}$, clear.

$09 \mathrm{Z}$ - Clear and around $6^{\circ} \mathrm{C}$ with light variable winds. Mountains around $-2^{\circ} \mathrm{C}$ with very light winds.

$12 \mathrm{Z}$ - Slightly cloudy to clear plains, around $2^{\circ} \mathrm{C}$ and southwest winds near $2 \mathrm{~m} \mathrm{~s}^{-1}$. High $1023 \mathrm{mb}$ southwest of Gunnison. Little statewide gradient.

Post case conditions: Clouding up, high near $10^{\circ} \mathrm{C}$. Winds up to $4.5 \mathrm{~m} \mathrm{~s}^{-1}$. No frontal passage though. 


\section{APPENDIX B}

\section{Froude Number Climatology 1966-1993}

This climatology of Froude numbers for the Colorado Front Range considers flows with a significant portion of their flow in the westerly component - those which would impact the Front Range Canyons of concern to this dissertation as a lee-side effect. The Froude number is discussed in Section 2.6 on page 65 . Froude number was calculated using two separate analysis methods, 1) where only the average u component was considered and 2) where only average winds whose wind direction fell within the range $235^{\circ}$ and $315^{\circ}$ (the directions bounded by true southwest and true northwest cardinal points). As described in Section 3.4 on page 106 averages were taken over a $2000 \mathrm{~m}$ range $(2000 \mathrm{~m}-4000 \mathrm{~m}$ MSL) corresponding to the barrier height, $\mathrm{H}$, that flow over the Colorado Continental Divide is forced to rise above. The data used for this analysis came from the twice daily (00Z and $12 Z$ ) rawinsonde launches from Grand Junction, Colorado west of the Divide at elevation $1450 \mathrm{~m}$ MSL over the 28 year period 1966-1993. This corresponds to a possible 20454 rawinsondes for analysis under ideal circumstances plus a few hundred special soundings. To create valid averages in the $2000 \mathrm{~m}-4000 \mathrm{~m}$ MSL interval only those soundings where there existed 2 or more points in this range for both $\theta$ and $u$ (or $\theta$ and V)were used. This eliminated 733 soundings from the $u$ wind based Froude number $\left(\mathrm{Fr}_{\mathrm{u}}\right)$ analysis. Considering only cases where $u$ was positive reduced the soundings available for 
analysis to 16421 . The predominance of westerly flow, within the cited range, over the Rockies can be approximately calculated from the ratio of the positive $\mathrm{u}$ cases to total cases giving $\sim 80 \%$ ! Aloft this value is undoubtedly even higher. Details are given below.

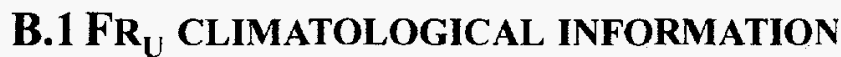

Average $\mathrm{Fr}_{\mathrm{u}}: 0.305$

Average $\mathrm{d} \theta / \mathrm{dz}: 2.742 \mathrm{~K} \mathrm{~km}^{-1}$

Average $O: 3.396 \mathrm{~m} \mathrm{~s}^{-1}$

Total number of soundings used (including special soundings): 20470

Number of easterly component cases (no special soundings): 3726 (18.5\%)

Number of westerly component cases (no special soundings): $16421(81.5 \%$ )

Number of special soundings: 323

Number of soundings not containing sufficient data for analysis: 303

Minimum positive $\mathrm{Fr}_{\mathrm{u}}: 1.76 \times 10^{-5}$ on $12 \mathrm{Z}$ MAR- 5-1993

Maximum $\mathrm{Fr}_{\mathrm{u}}: 30.54$ on $00 \mathrm{Z}$ OCT- $4-1967$

$\mathrm{Fr}_{\mathrm{u}}$ for westerly component: .353

$\mathrm{Fr}_{\mathrm{u}}$ for easterly component: -.099

Average $\mathrm{Fr}_{\mathrm{u}}$ at $12 \mathrm{Z}: \quad 0.217$

Average $\mathrm{d} \theta / \mathrm{dz}$ at $12 \mathrm{Z}: 3.33 \mathrm{~K} \mathrm{~km}^{-1}$

Average $O$ at $12 \mathrm{Z}: 2.99 \mathrm{~m} \mathrm{~s}^{-1}$

Total number of soundings used: 9963

Average $\mathrm{Fr}_{\mathrm{u}}$ at $00 \mathrm{Z}: \quad 0.397$

Average $\mathrm{d} \theta / \mathrm{dz}$ at $00 \mathrm{Z}: 2.12 \mathrm{~K} \mathrm{~km}^{-1}$

Average $O$ at $00 \mathrm{Z}: 3.81 \mathrm{~m} \mathrm{~s}^{-1}$

Total number of soundings used: 9558 
Table 6.5: Average $\mathrm{Fr}_{\mathrm{u}}$ by month of year

\begin{tabular}{|c|c|c|c|c|c|}
\hline Month & $\begin{array}{c}\text { Average } \\
\mathrm{Fr}_{\mathrm{u}}\end{array}$ & $\begin{array}{c}\text { Standard } \\
\text { deviation }\end{array}$ & $\begin{array}{c}\text { Avg. } \mathrm{Fr}_{\mathrm{u}} \\
00 \mathrm{Z} \text { only }\end{array}$ & $\begin{array}{c}\text { Avg. } \mathrm{Fr}_{\mathrm{u}} \\
12 \mathrm{Z} \text { only }\end{array}$ & $\begin{array}{c}\Delta \mathrm{Fr}_{\mathrm{u}} \\
(00 \mathrm{Z}-12 \mathrm{Z})\end{array}$ \\
\hline \hline January & 0.22 & 0.18 & 0.24 & 0.19 & 0.05 \\
\hline February & 0.25 & 0.21 & 0.28 & 0.22 & 0.06 \\
\hline March & 0.35 & 0.29 & 0.41 & 0.29 & 0.12 \\
\hline April & 0.47 & 0.42 & 0.64 & 0.31 & 0.33 \\
\hline May & 0.38 & 0.34 & 0.54 & 0.24 & 0.30 \\
\hline June & 0.42 & 0.39 & 0.64 & 0.23 & 0.31 \\
\hline July & 0.29 & 0.30 & 0.45 & 0.15 & 0.30 \\
\hline August & 0.27 & 0.27 & 0.41 & 0.14 & 0.27 \\
\hline September & 0.32 & 0.29 & 0.44 & 0.21 & 0.23 \\
\hline October & 0.26 & 0.24 & 0.33 & 0.20 & 0.13 \\
\hline November & 0.25 & 0.22 & 0.28 & 0.22 & 0.06 \\
\hline December & 0.21 & 0.18 & 0.22 & 0.20 & 0.02 \\
\hline
\end{tabular}

Table 6.6: Average thermal stabililty and wind speed below barrier by month of year

\begin{tabular}{|c|c|c|c|c|c|c|}
\hline Month & $\begin{array}{c}\text { Average. } \frac{\partial \theta}{\partial z} \\
\left(\mathrm{~K} \mathrm{~km}^{-1}\right)\end{array}$ & $\begin{array}{c}\text { Average. } \frac{\partial \theta}{\partial z} \\
\text { at 00Z } \\
\left(\mathrm{K} \mathrm{km}^{-1}\right)\end{array}$ & $\begin{array}{c}\text { Average. } \frac{\partial \theta}{\partial z} \\
\text { at } 12 Z \\
\left(\mathrm{~K} \mathrm{~km}^{-1}\right)\end{array}$ & $\begin{array}{c}\text { Average u } \\
\text { wind speed } \\
\left(\mathrm{m} \mathrm{s}^{-1}\right)\end{array}$ & $\begin{array}{c}\text { Average u } \\
\text { at 00Z } \\
\left(\mathrm{m} \mathrm{s}^{-1}\right)\end{array}$ & $\begin{array}{c}\text { Average u } \\
\text { at 12Z } \\
\left(\mathrm{m} \mathrm{s}^{-1}\right)\end{array}$ \\
\hline \hline January & 5.11 & 4.82 & 5.40 & 4.12 & 4.37 & 3.87 \\
\hline February & 3.57 & 3.20 & 3.96 & 3.76 & 4.01 & 3.54 \\
\hline March & 2.29 & 1.84 & 2.69 & 4.05 & 4.22 & 3.94 \\
\hline April & 1.74 & 1.08 & 2.36 & 4.03 & 4.42 & 3.66 \\
\hline May & 1.65 & 0.96 & 2.29 & 3.41 & 4.08 & 2.78 \\
\hline June & 1.66 & 0.73 & 2.50 & 3.23 & 3.99 & 2.55 \\
\hline July & 1.77 & 0.75 & 2.71 & 2.03 & 2.75 & 1.37 \\
\hline August & 1.76 & 0.81 & 2.65 & 2.12 & 2.70 & 1.58 \\
\hline September & 1.95 & 1.08 & 2.75 & 3.03 & 3.60 & 2.46 \\
\hline October & 2.53 & 1.84 & 3.23 & 3.06 & 3.40 & 2.74 \\
\hline November & 3.64 & 3.14 & 4.13 & 3.87 & 4.11 & 3.59 \\
\hline December & 4.91 & 4.56 & 5.25 & 3.99 & 4.07 & 3.84 \\
\hline
\end{tabular}




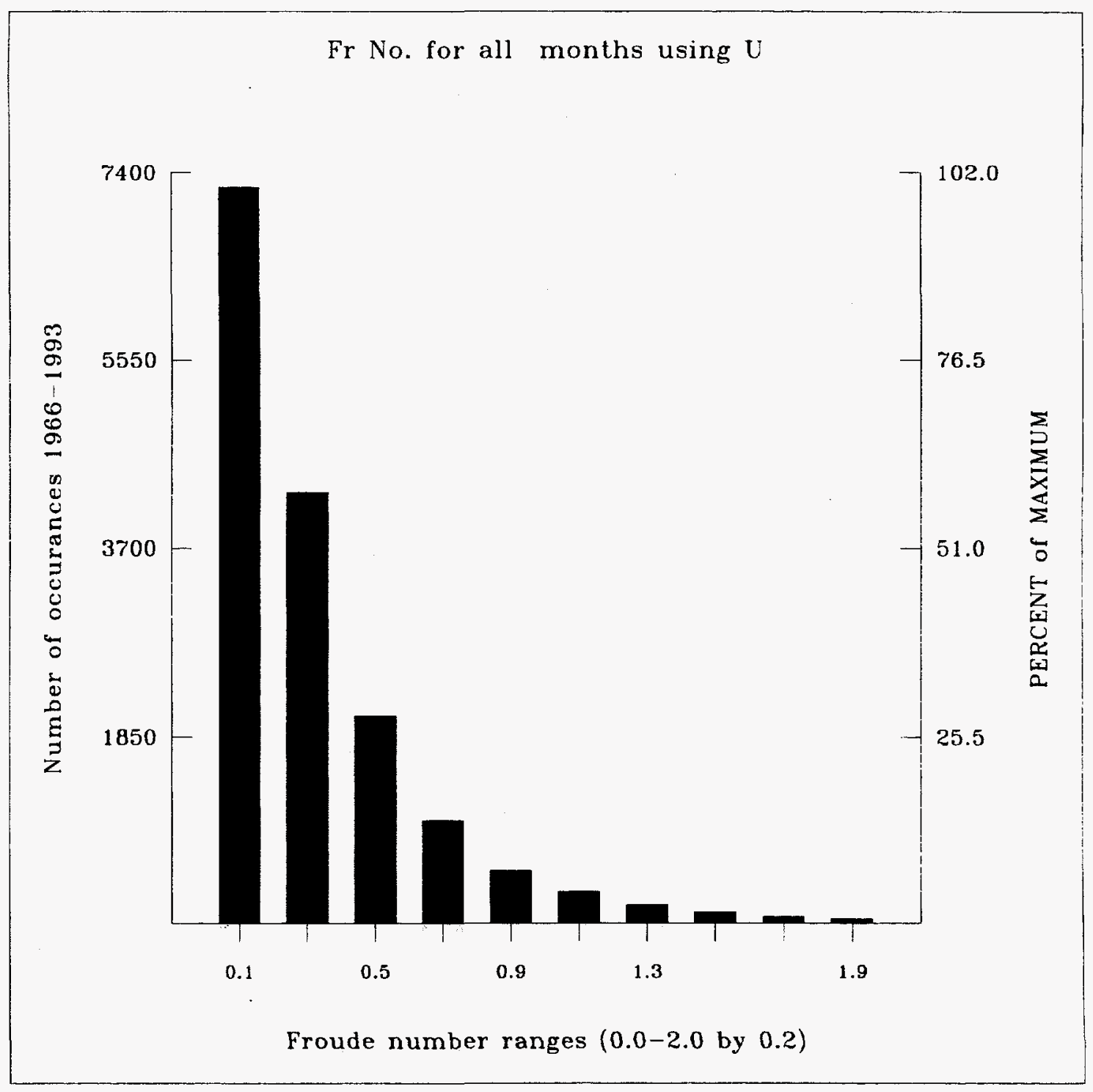

Figure B.1 Froude number histogram based on all soundings at Grand Junction for the period 1966-1993. Only the u component was used for flow over a $2000 \mathrm{~m}$ barrier 


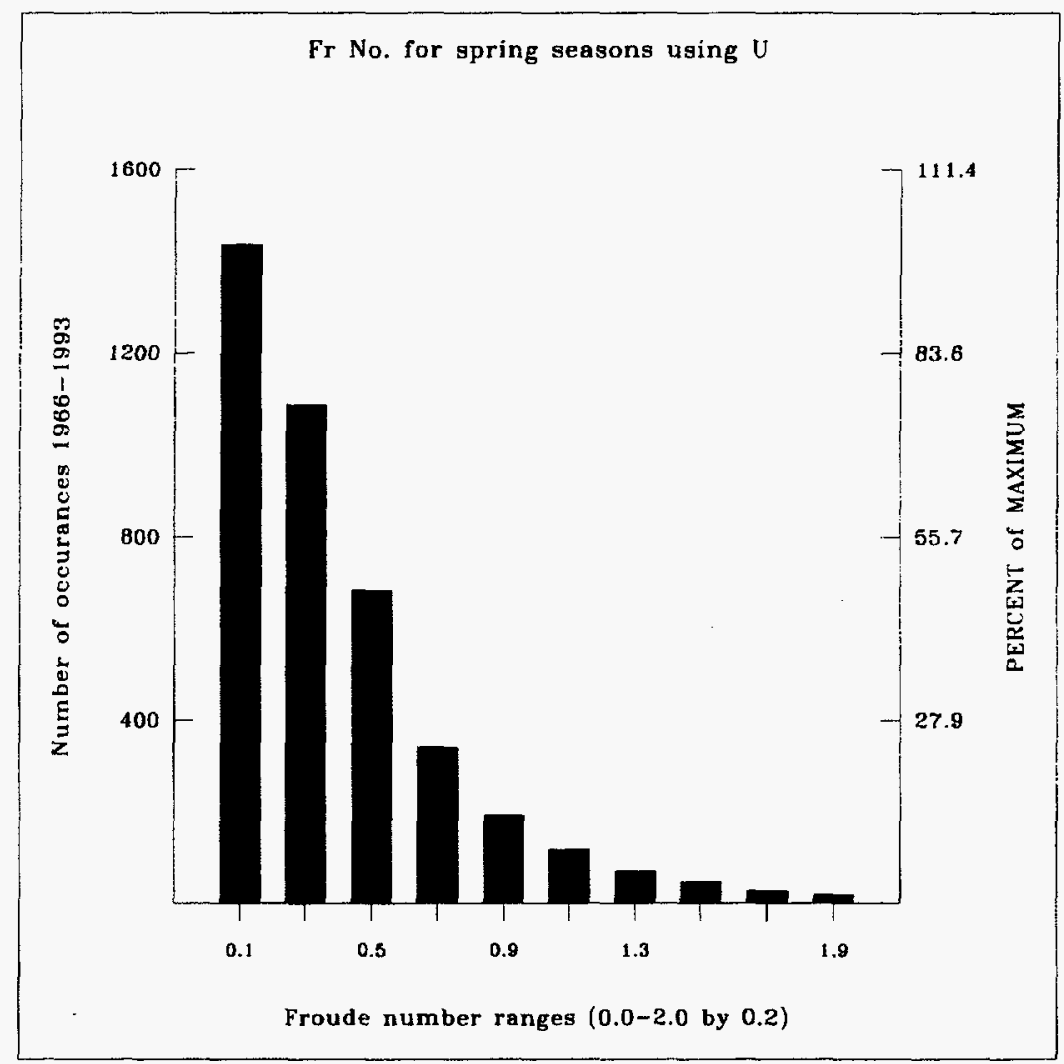

Figure B.2 .As in Figure B.1 but limited to the spring season (March-April-May).

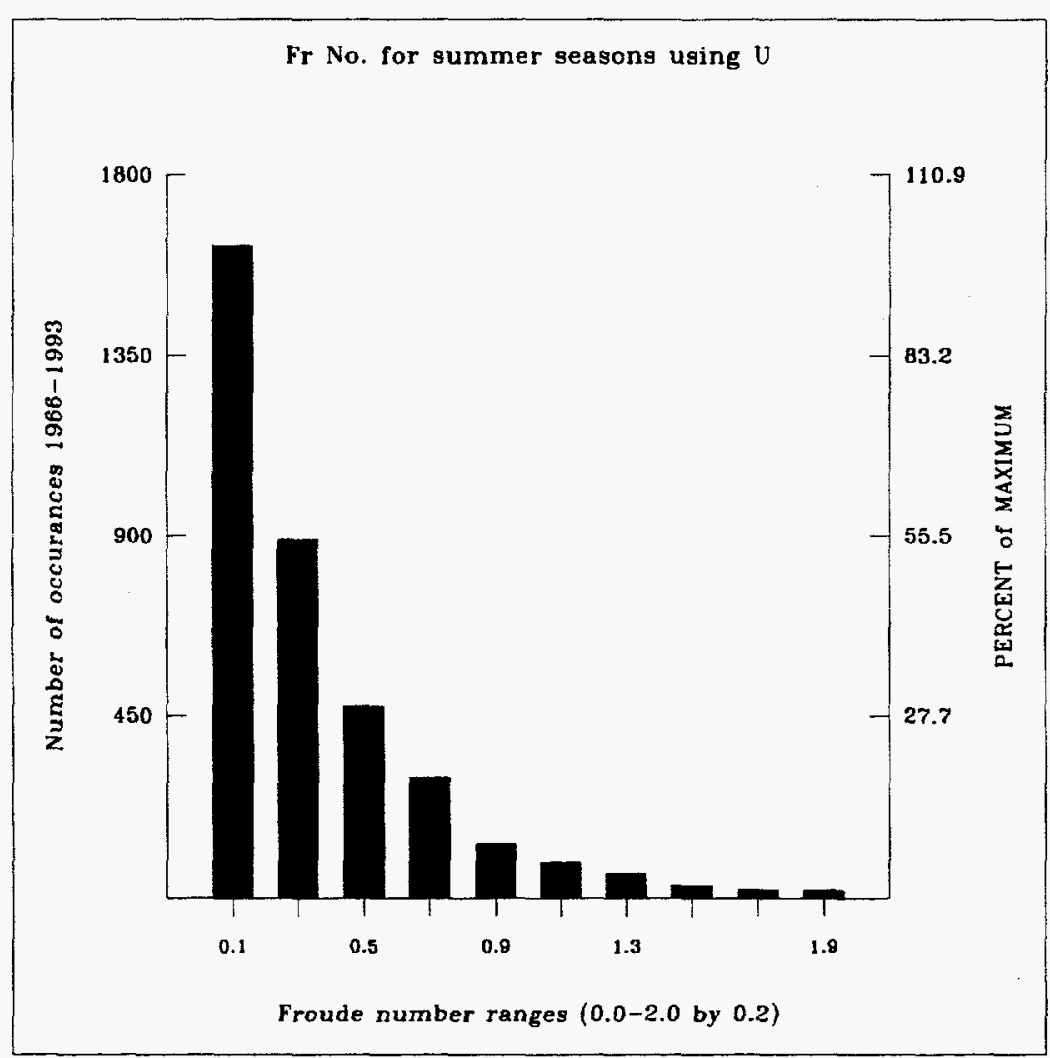

Figure B.3 As in Figure B.1 but for the summer season.. 


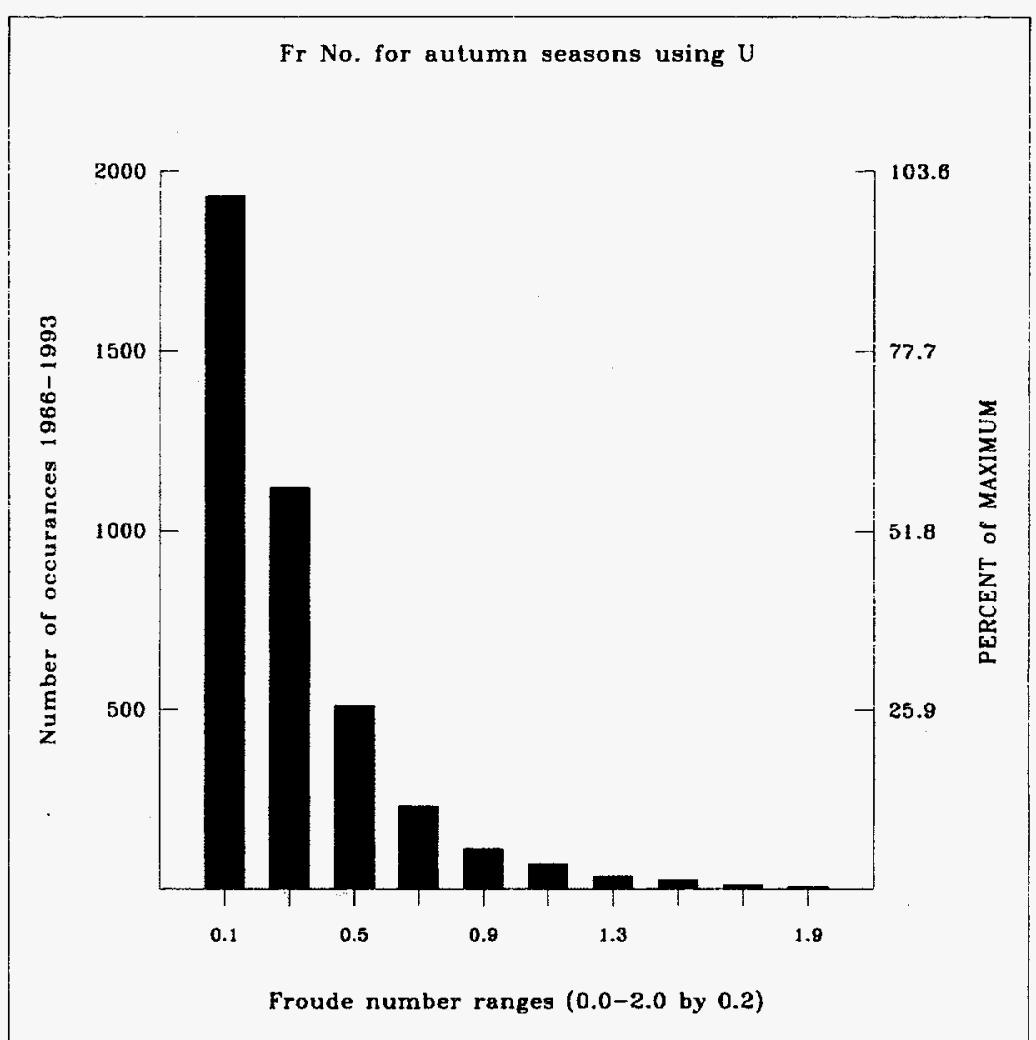

Figure B.4 As in Figure B.1 but for autumn.

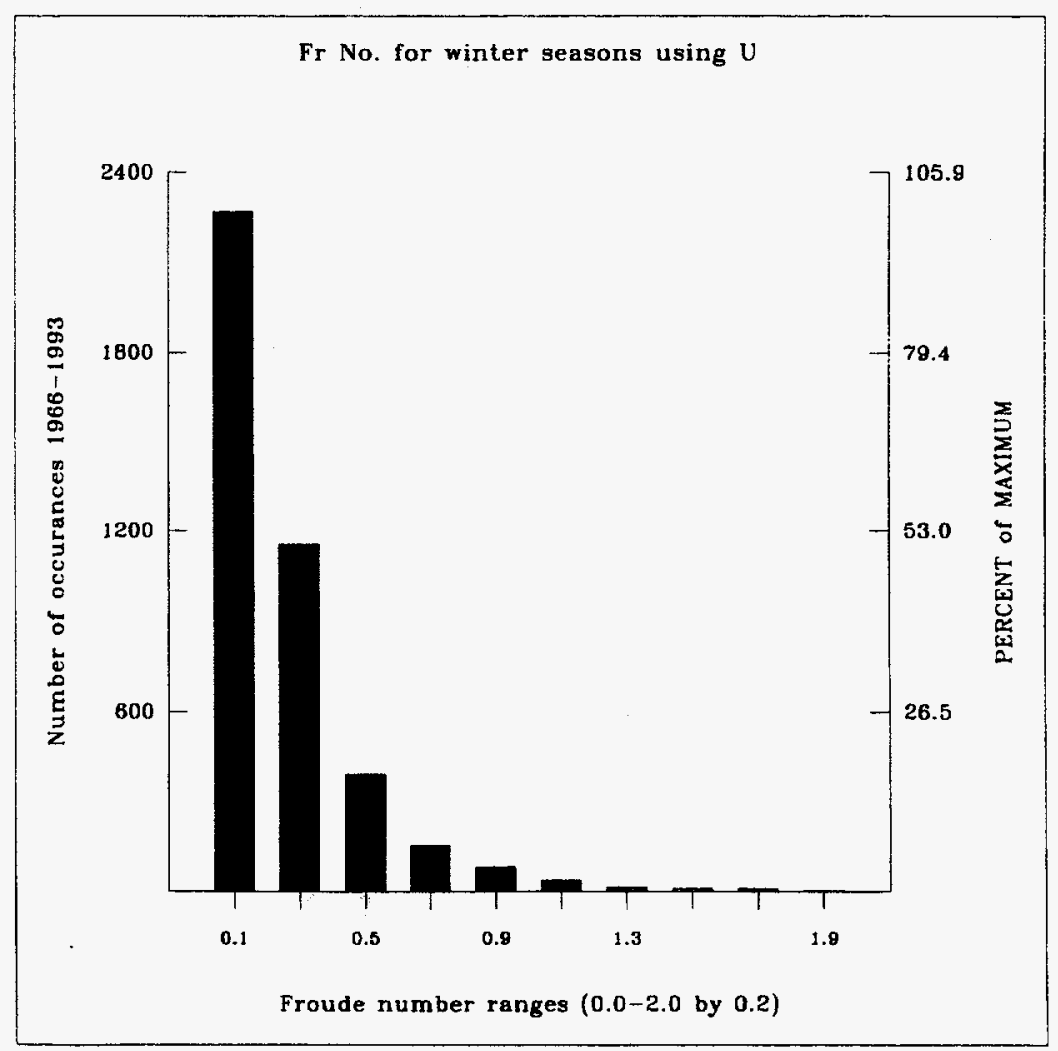

Figure B.5 As in Figure B.1 but for the winter season . 


\section{B.2 $\mathrm{FR}_{\mathrm{V}}$ CLIMATOLOGICAL INFORMATION}

Average $\mathrm{Fr}_{\mathrm{v}}: .63 \dot{2}$

Number of soundings passing selection criteria: 5591

Average $\mathrm{Fr}_{\mathrm{v}}$ at $12 \mathrm{Z}: 0.533$

Average $\mathrm{Fr}_{\mathrm{v}}$ at $00 \mathrm{Z}: 0.686$

Min imum $\mathrm{Fr}_{\mathrm{v}}: 0.043$ on 00Z JAN-30-1976

Maximum $\mathrm{Fr}_{\mathrm{v}}: 24.760$ on $00 \mathrm{Z}$ APR-15-1970

Table 6.7: Average $\mathrm{Fr}_{\mathrm{v}}$ by month of year

\begin{tabular}{|c|c|c|c|c|c|}
\hline Month & $\begin{array}{c}\text { Average } \\
\mathrm{Fr}_{v}\end{array}$ & $\begin{array}{c}\text { Standard } \\
\text { deviation }\end{array}$ & $\begin{array}{c}\text { Avg. Fr } \\
00 \mathrm{Z} \text { only }\end{array}$ & $\begin{array}{c}\text { Avg. Fr } \\
12 \mathrm{Z} \text { only }\end{array}$ & $\begin{array}{c}\Delta \mathrm{Fr}_{\mathrm{v}} \\
(00 \mathrm{Z}-\mathrm{I} 2 \mathrm{Z})\end{array}$ \\
\hline \hline January & 0.42 & 0.22 & 0.42 & 0.43 & 0.01 \\
\hline February & 0.48 & 0.26 & 0.49 & 0.47 & 0.02 \\
\hline March & 0.66 & 0.36 & 0.69 & 0.62 & 0.07 \\
\hline April & 0.88 & 0.55 & 1.03 & 0.64 & 0.39 \\
\hline May & 0.76 & 0.39 & 0.85 & 0.57 & 0.28 \\
\hline June & 0.88 & 0.50 & 1.02 & 0.58 & 0.44 \\
\hline July & 0.76 & 0.39 & 0.82 & 0.55 & 0.27 \\
\hline August & 0.65 & 0.36 & 0.73 & 0.39 & 0.34 \\
\hline September & 0.68 & 0.33 & 0.72 & 0.58 & 0.14 \\
\hline October & 0.49 & 0.24 & 0.50 & 0.48 & 0.02 \\
\hline November & 0.51 & 0.26 & 0.50 & 0.53 & -0.03 \\
\hline December & 0.42 & 0.21 & 0.38 & 0.49 & -0.11 \\
\hline
\end{tabular}


Table 6.8: Average thermal stabililty and $V$ wind speed below barrier by month of year

\begin{tabular}{|c|c|c|c|c|c|c|}
\hline Month & $\begin{array}{c}\text { Average } \frac{\partial \theta}{\partial z} \\
\left(\mathrm{~K} \mathrm{~km}^{-1}\right)\end{array}$ & $\begin{array}{c}\text { Average } \frac{\partial \theta}{\partial z} \\
\text { at } 00 \mathrm{Z} \\
\left(\mathrm{K} \mathrm{km}^{-1}\right)\end{array}$ & $\begin{array}{c}\text { Average } \frac{\partial \theta}{\partial z} \\
\text { at } 12 \mathrm{Z} \\
\left(\mathrm{K} \mathrm{km}^{-1}\right)\end{array}$ & $\begin{array}{c}\text { Average V } \\
\text { wind speed } \\
\left(\mathrm{m} \mathrm{s}^{-1}\right)\end{array}$ & $\begin{array}{c}\text { Average } \mathrm{V} \\
\text { at 00Z } \\
\left(\mathrm{m} \mathrm{s}^{-1}\right)\end{array}$ & $\begin{array}{c}\text { Average V } \\
\text { at 12Z } \\
\left(\mathrm{m} \mathrm{s}^{-1}\right)\end{array}$ \\
\hline \hline January & 5.27 & 5.39 & 5.08 & 9.07 & 8.66 & 9.78 \\
\hline February & 3.59 & 3.56 & 3.69 & 8.19 & 7.72 & 9.00 \\
\hline March & 2.21 & 2.00 & 2.50 & 8.33 & 7.50 & 9.56 \\
\hline April & 1.55 & 1.09 & 2.24 & 7.95 & 7.42 & 8.74 \\
\hline May & 1.41 & 1.04 & 2.13 & 7.50 & 7.17 & 8.13 \\
\hline June & 1.23 & 0.81 & 2.06 & 7.21 & 6.64 & 8.31 \\
\hline July & 1.04 & 0.72 & 2.08 & 6.14 & 5.65 & 7.78 \\
\hline August & 1.19 & 0.84 & 2.34 & 5.52 & 5.30 & 6.23 \\
\hline September & 1.37 & 1.07 & 2.20 & 6.96 & 6.35 & 8.71 \\
\hline October & 2.46 & 2.16 & 3.02 & 7.07 & 6.41 & 8.36 \\
\hline November & 3.50 & 3.38 & 3.69 & 8.79 & 8.17 & 9.76 \\
\hline December & 4.96 & 5.22 & 4.51 & 8.92 & 7.87 & 10.37 \\
\hline
\end{tabular}




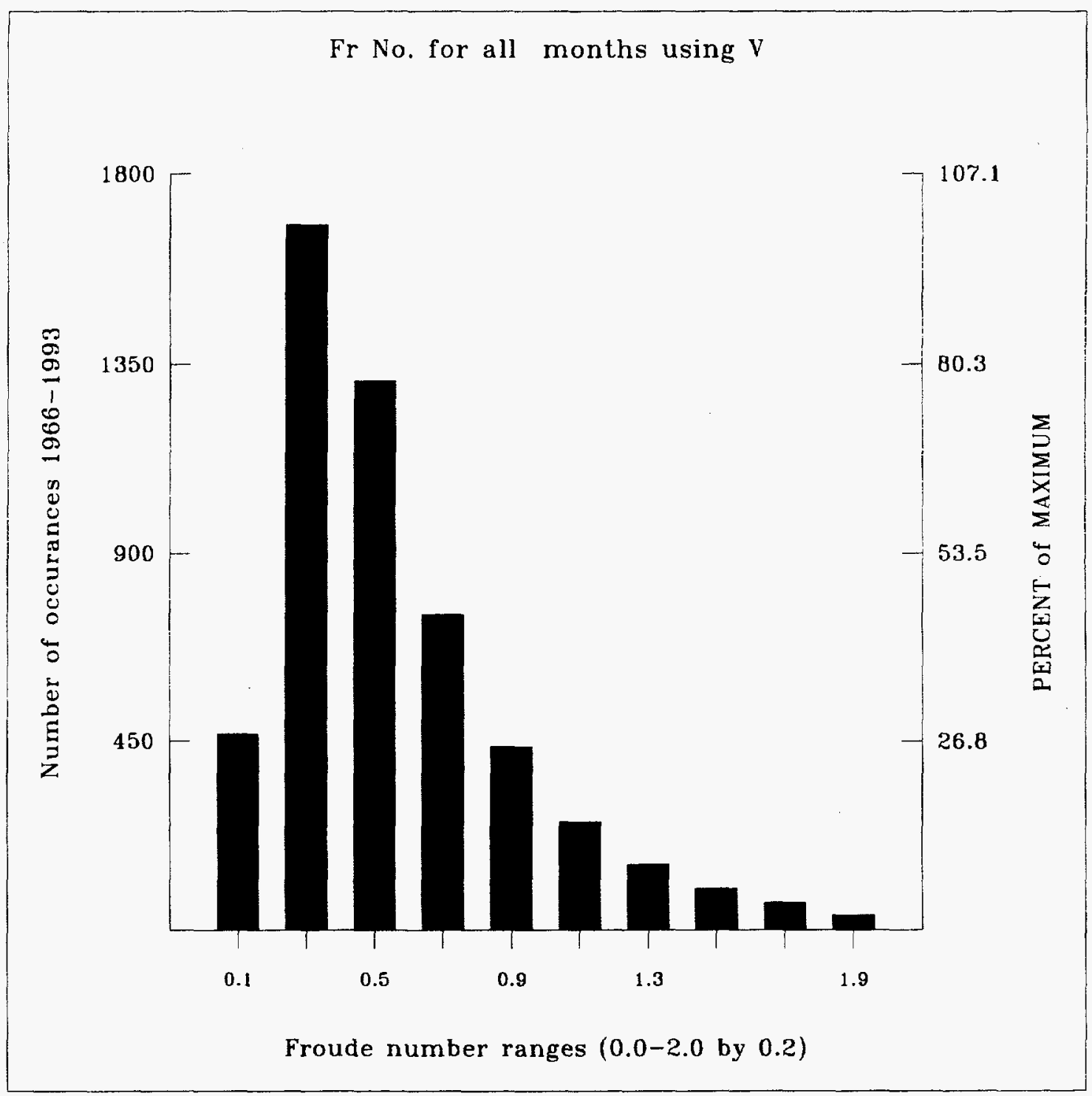

Figure B.6 As in Figure B.1 but for the horizontal wind, V, for only those days where the westerly componen was at least half the flow (i.e. wind direction limited to the $235^{\circ}-315^{\circ}$ range.). 


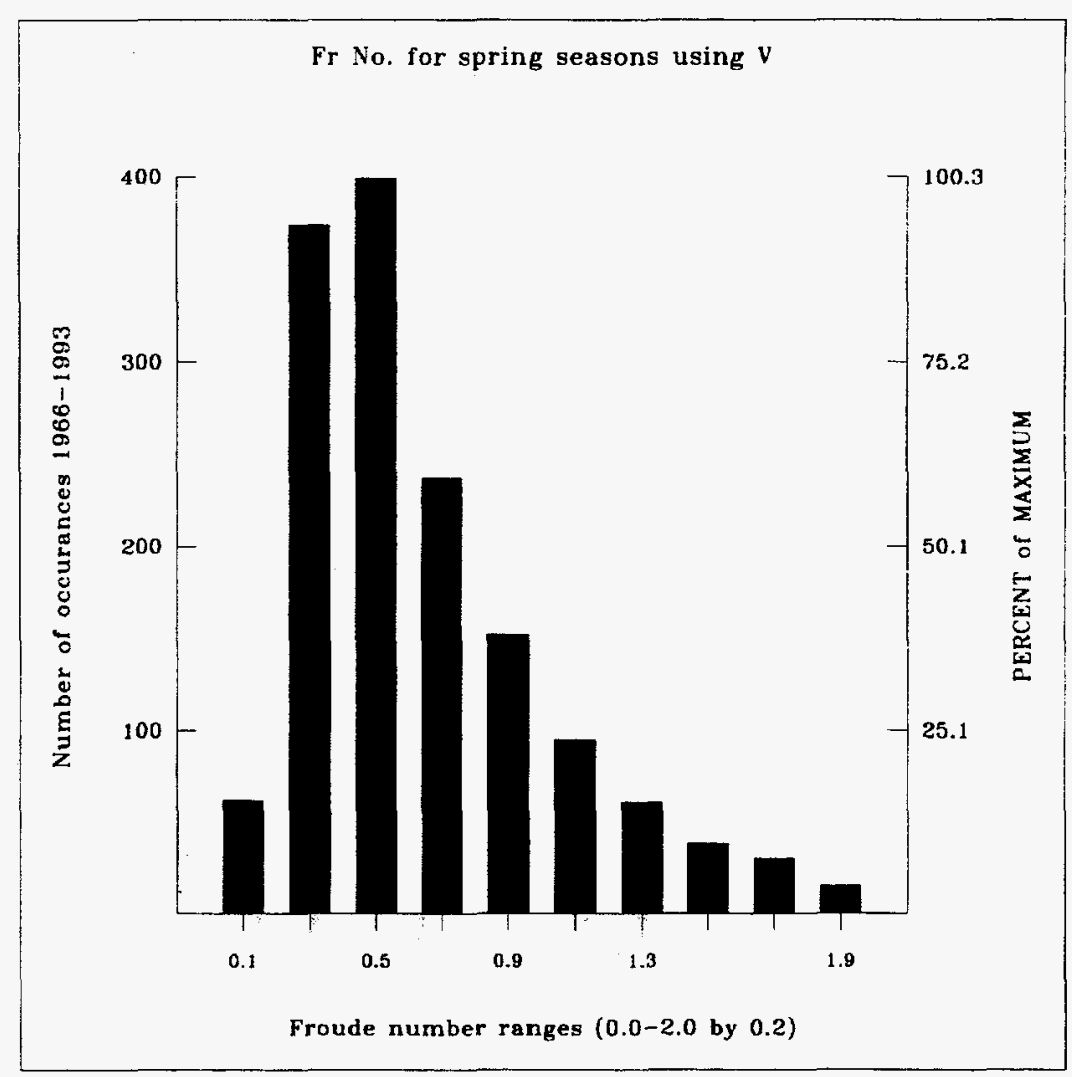

Figure B.7 As in Figure B.1 but for the spring season only .

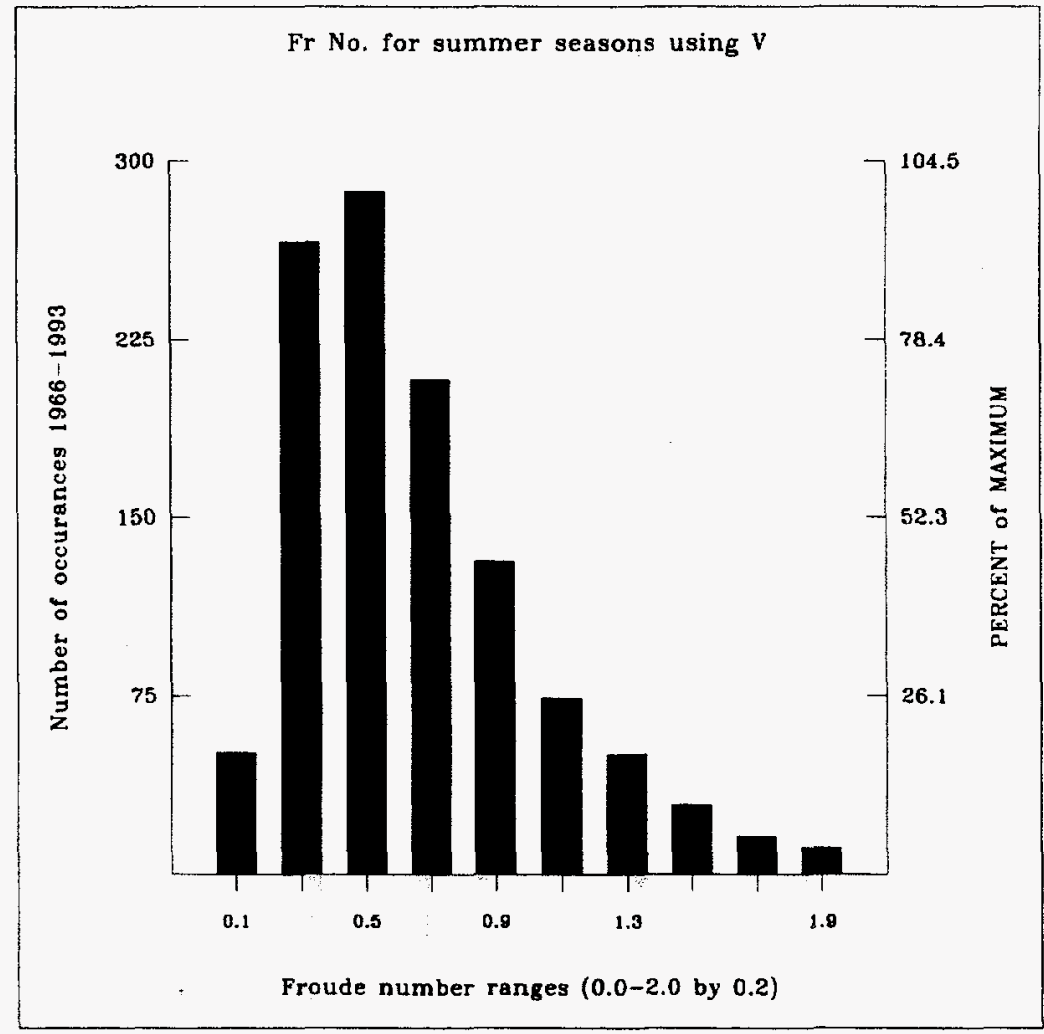

Figure B.8 As in Figure B.1 but for the summer season.. 
Fr No. for autumn seasons using $V$

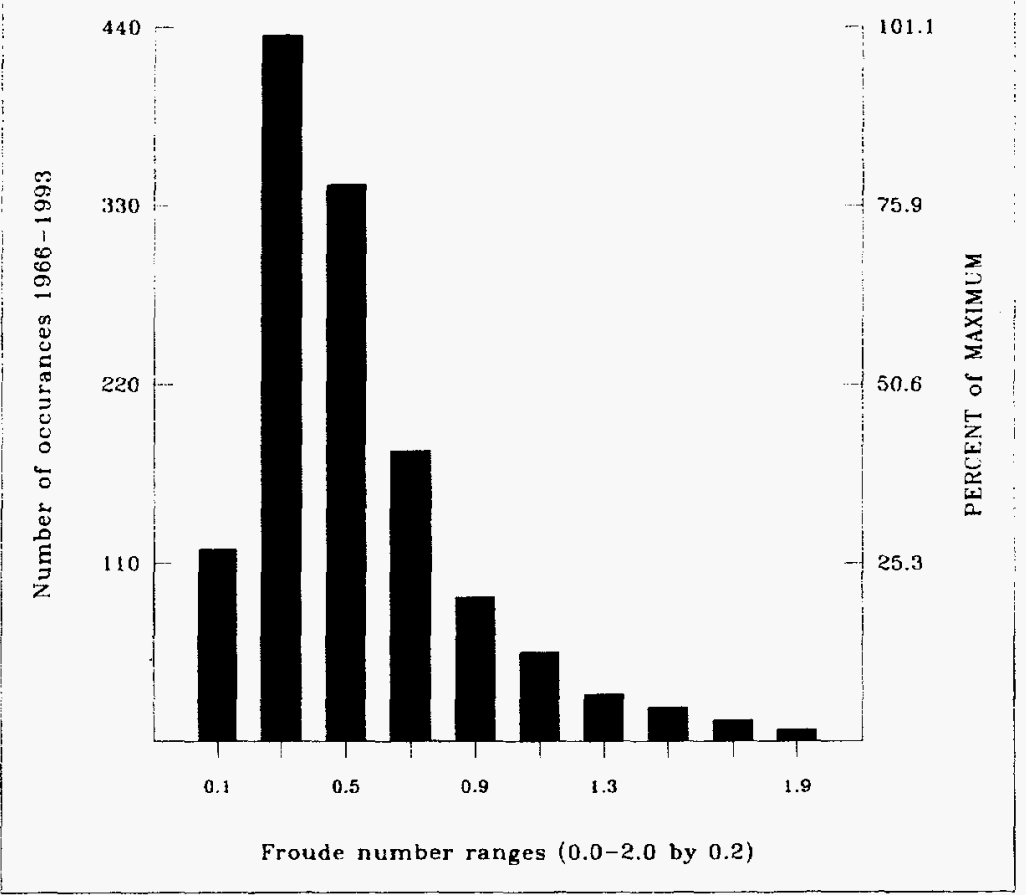

Figure B.9 As in Figure B.1 but for autumn..

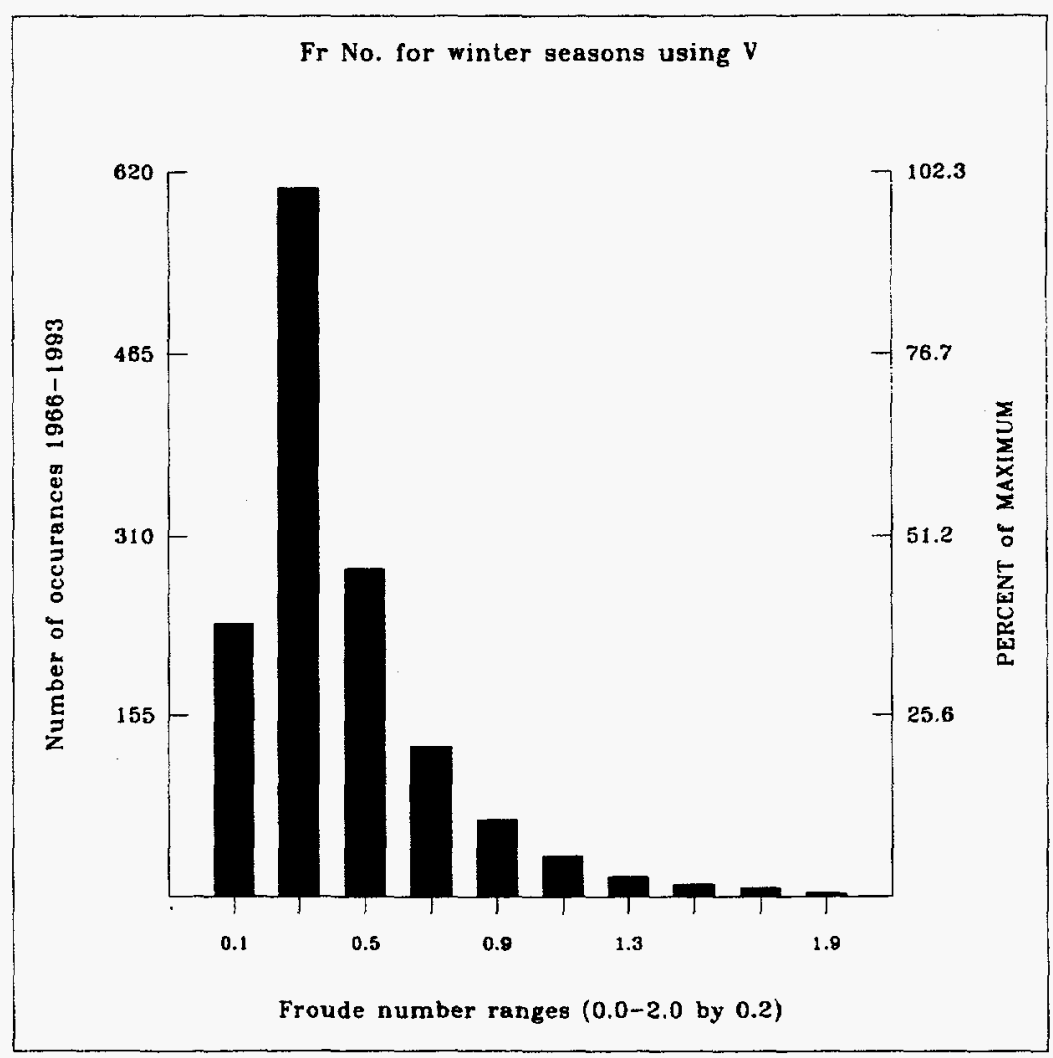

Figure B.10 As in Figure B.1 but for winter. 
Gregory S. Poulos

300 